\title{
EPIGENETIC MORPHOLOGY AND CLASSIFICATION \\ OF NEUROENDOCRINE TUMORS
}

\author{
Wenzel M. Hackeng
}

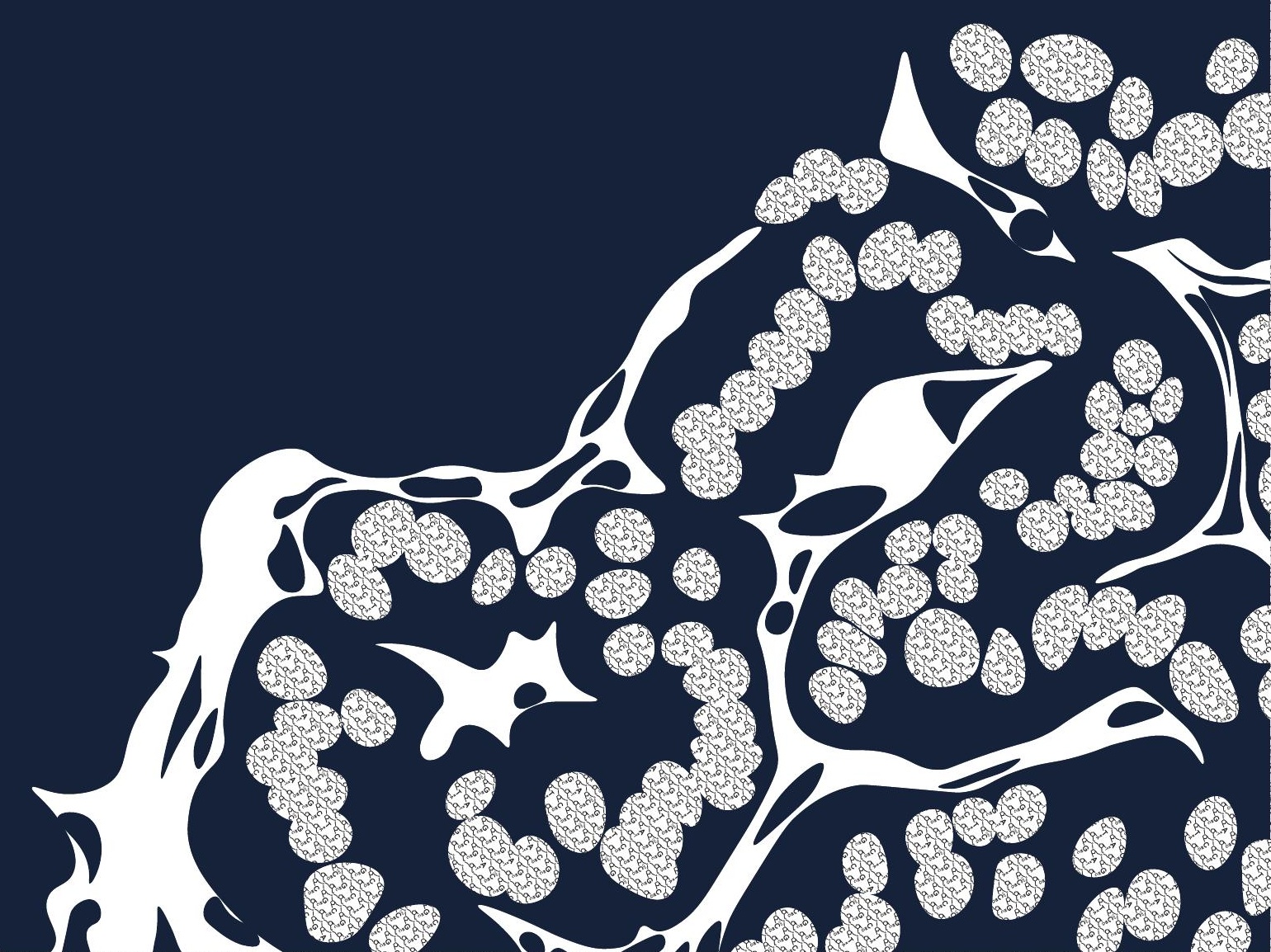





\section{EPIGENETIC MORPHOLOGY AND CLASSIFICATION OF NEUROENDOCRINE TUMORS}

Academic thesis, Utrecht University, The Netherlands

ISBN: 9789464193404

Print: Gildeprint, Enschede

Cover Layout: W. M. Hackeng

Cover Title "Epigenetic Zebra Morphology"

Financial support for printing this thesis was kindly provided by the Nederlandse Vereniging voor Gastro-enterologie (NVGE) and Ipsen

Copyright 2021 W. M. Hackeng.

For articles published, copyright may have been transferred to the respective publisher. No parts of this thesis may be reproduced, stored in a retrieval system, or transmitted in any form or by any means, without prior written permission of the author, or when appropriate, from the publisher. 


\section{Epigenetic Morphology and Classification of Neuroendocrine Tumors}

Epigenetische morfologie en classificatie van neuro-endocriene tumoren

(met een samenvatting in het Nederlands)

\section{Proefschrift}

ter verkrijging van de graad van doctor aan de Universiteit Utrecht op gezag van de rector magnificus, prof.dr. H.R.B.M. Kummeling, ingevolge het besluit van het college voor promoties in het openbaar te verdedigen op donderdag 20 januari 2022 des middags te 2.15 uur

door

Wenzel Maximilian Hackeng

geboren op 13 januari 1992

te Utrecht 


\section{Promotor:}

Prof. dr. G. J. A. Offerhaus

\section{Copromotoren:}

Dr. L. A. A. Brosens

Dr. K. M. A. Dreijerink

Dit proefschrift werd (mede) mogelijk gemaakt met financiële steun van de Maag Lever Darm stichting (MLDS) 


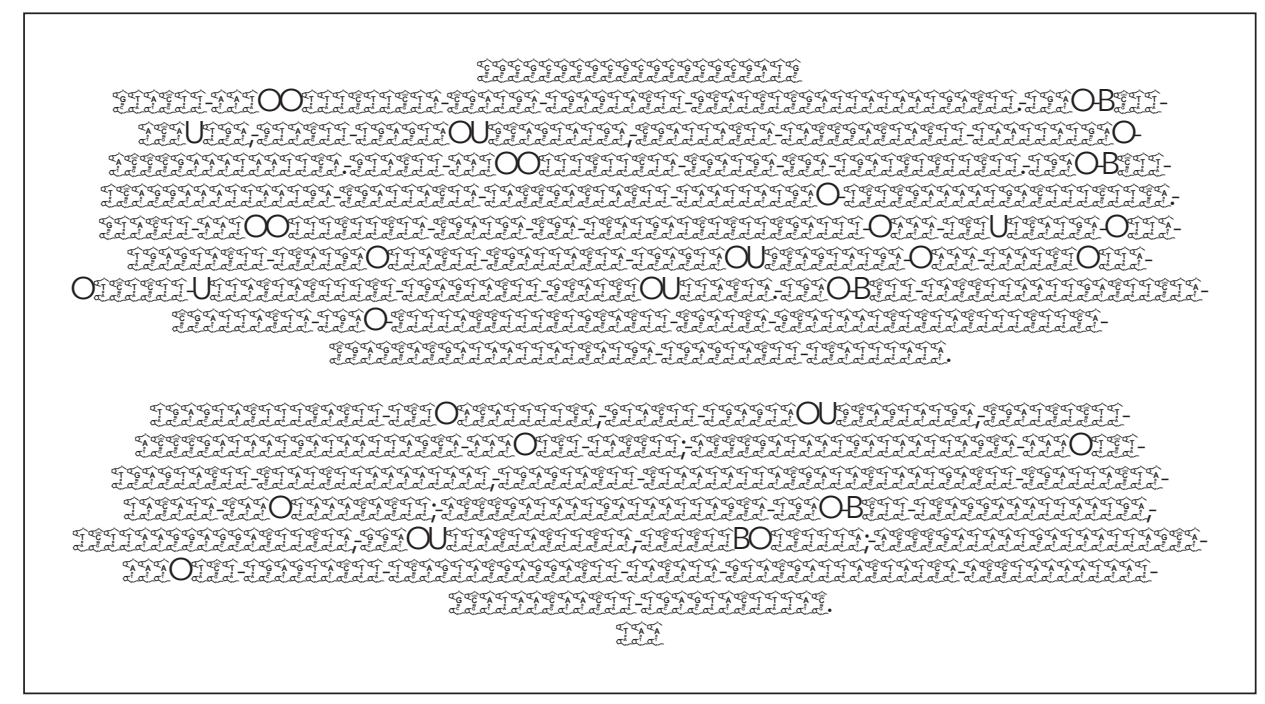

Aan mijn ouders

Aan Anne 


\section{CONTENTS}

CHAPTER 1 INTRODUCTION

\section{PART 1. CELL-DIFFERENTIATION SPECIFIC DUODENOPANCREATIC TUMORIGENIC PATHWAYS}

CHAPTER 2 SURGICAL AND MOLECULAR PATHOLOGY OF PANCREATIC NEOPLASMS Diagnostic Pathology, 2016

CHAPTER 3 ABERRANT MENIN EXPRESSION IS AN EARLY EVENT IN PANCREATIC NEUROENDOCRINE TUMORIGENESIS

Human Pathology, 2016

CHAPTER 4 GLUCAGON CELL HYPERPLASIA AND NEOPLASIA

Encyclopedia of Pathology, 2020

CHAPTER 5 A PARATHYROID-GUT AXIS: HYPERCALCEMIA AND THE PATHOGENESIS OF GASTRINOMA IN MULTIPLE ENDOCRINE NEOPLASIA 1

Molecular Cancer Research, 2021

\section{PART 2. EPIGENETIC DIFFERENTIATION REFINES NEUROENDOCRINE TUMOR CLASSIFICATION}

CHAPTER 6 ASSESSMENT OF ARX EXPRESSION, A NOVEL BIOMARKER FOR METASTATIC RISK IN PANCREATIC NEUROENDOCRINE TUMORS, IN ENDOSCOPIC ULTRASOUND FINE NEEDLE ASPIRATION

Diagnostic Cytopathology, 2019

CHAPTER 7 ARX PROTEIN EXPRESSION AND ALTERNATIVE LENGTHENING OF TELOMERES IDENTIFY MALIGNANT INSULINOMAS

Endocrine Pathology, 2019

CHAPTER 8 NON-FUNCTIONAL PANCREATIC NEUROENDOCRINE TUMORS: ATRX/DAXX AND ALTERNATIVE LENGTHENING OF TELOMERES (ALT) ASSESS PROGNOSIS INDEPENDENTLY FROM ISLET-CELL SUBTYPE AND TUMOR SIZE Gut, 2021

CHAPTER 9 METASTATIC PATTERNS OF DUODENOPANCREATIC NEUROENDOCRINE TUMORS IN PATIENTS WITH MULTIPLE ENDOCRINE NEOPLASIA TYPE 1 American Journal of Diagnostic Pathology, 2021 
CHAPTER 10 GENOME METHYLATION ACCURATELY PREDICTS NEUROENDOCRINE TUMOR ORIGIN - AN ONLINE TOOL

Clinical Cancer Research, 2020

CHAPTER 11 SUMMARIZING DISCUSSION

\section{APPENDICES}

ABBREVIATIONS

DUTCH SUMMARY/NEDERLANDSE SAMENVATTING

CONTRIBUTING AUTHORS

CURRICULUM VITAE

LIST OF PUBLICATIONS

ACKNOWLEDGEMENTS/DANKWOORD 

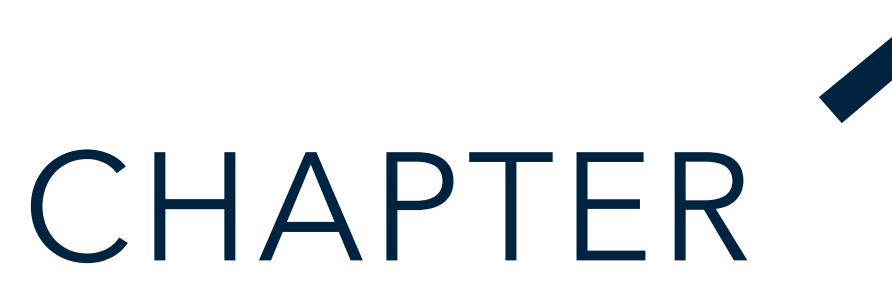

Introduction 


\section{NET KNOWLEDGE GAPS AND UNMET NEEDS DISCUSSED IN THIS THESIS}

- The sequence of events leading to duodenopancreatic neuroendocrine tumorigenesis.

- $\quad$ Pre- and postoperative prognostic biomarkers to predict behavior of pancreatic neuroendocrine tumors.

- $\quad$ Biomarkers to predict primary origin of neuroendocrine tumor metastases of unknown/uncertain primary site. 
Well-differentiated neuroendocrine tumors are rare tumors with an incidence of 6 per 100,000 per year. Neuroendocrine tumors can arise in any tissue containing endocrine cells, but are most commonly seen in the lung, pancreas, small-intestines or colorectum (1) (Figure 1). Microscopically, neuroendocrine tumors can be recognized by a typical trabecular (this thesis' cover) or nested morphology (2). Neuroendocrine tumor cells contain a dense granular cytoplasm, morphologically similar to synaptic granules, contributing to their "neural" designation, and a finely speckled "salt and pepper like" nuclear chromatin pattern (3). The cytoplasmic granules contain endocrine hormones, usually the same hormones produced naturally by endocrine cells from the tissue in which the neuroendocrine tumors arise. If secretion of these hormones becomes symptomatic, a neuroendocrine tumor is labeled "functional" and is sometimes named after its respective hormone (e.g., an insulin secreting neuroendocrine tumor in the pancreas causing hypoglycemic events is called an "insulinoma"). A non-functional neuroendocrine tumor may contain or secrete hormones, but is per definition non-symptomatic.

Ourunderstanding of the sequence of events leading to duodenopancreatic neuroendocrine tumorigenesis has largely been based on the tumor-initiating mechanisms observed in hereditary neuroendocrine tumor syndromes, which include Multiple Endocrine Neoplasia 1 (MEN1), Von Hippel Lindau (VHL) and Glucagon Cell Hyperplasia and Neoplasia (GCHN)syndrome (5-8). In MEN1- and VHL-syndrome, a germline defect in the respective gene and loss of wildtype allele generally initiates neuroendocrine tumorigenesis. A different mechanism is seen in GCHN, in which neuroendocrine cell hyperplasia increases the risk of somatic mutations and subsequent neoplasia. In the non-syndromic (sporadic) tumorigenesis, the drivers initiating tumorigenesis may be similar to those in the syndromic tumorigenesis, as a proportion of the sporadic tumors harbor the same mutations (e.g., MEN1). Whole exome/genome sequencing studies identified the major oncogenic driver genes in sporadic pancreatic neuroendocrine tumors, including ARID1A, ATRX, CDKN1B, CDKN2A, DAXX, MEN1, SETD2, TP53, VHL, YY1 and genes in the mTOR pathway (9-12). However, the exact timing of events remains to be studied.

In addition to the knowledge gap that exists in our fundamental understanding of the mechanisms of tumorigenesis, several clinical challenges exist in the diagnosis, surgical decision-making and follow-up of patients with neuroendocrine tumors. Among neuroendocrine tumors from different origins, pancreatic neuroendocrine tumors have the highest risk of mortality $(1,4)$. Around half of patients have metastatic disease at diagnosis 


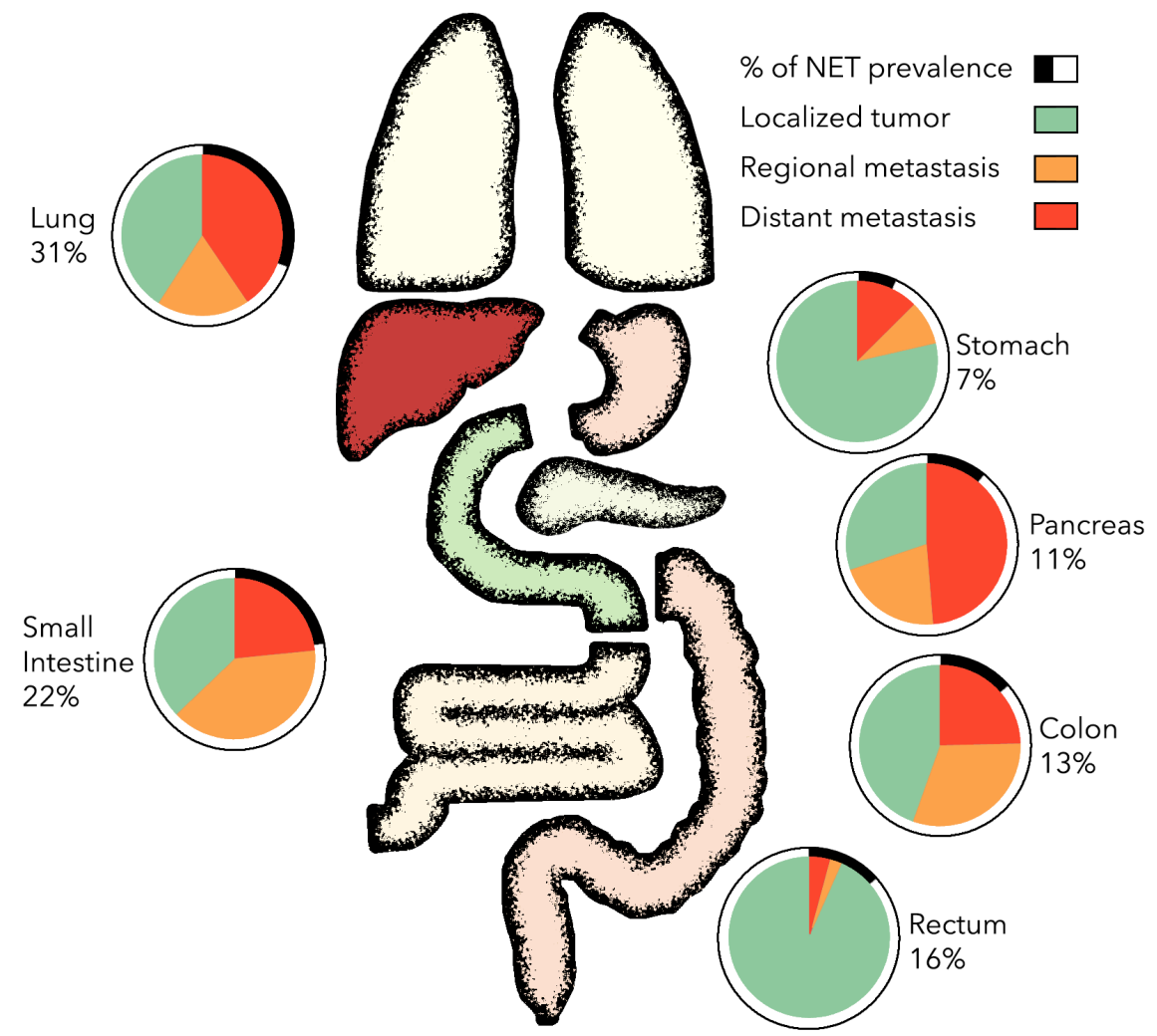

Figure 1. Neuroendocrine tumors (NETs) can arise in any organ, but are most often seen in the lung, small intestine, colorectum and pancreas (\% of NET prevalence). NETs arising in different organs have different proportions of localized (only in primary organ), regional (local tumor positive lymph nodes) and distant disease (distant organ metastases). Pancreatic NETs metastasize most often. The figure is based on Surveillance, Epidemiology, and End Results (SEER) data from the USA, extracted by Man et al. (4).

(Figure 1), and liver failure due to hepatic dissemination is the main cause of death (13). Guidelines recommend surgery based on size $(>2 \mathrm{~cm})$, presence of lymph node metastasis, higher grade or functional hormone production $(14,15)$. However, for small $(<1-2 \mathrm{~cm})$ nonfunctional pancreatic neuroendocrine tumors, the risk of metastases is low, and pancreatic surgery remains controversial as it is associated with significant morbidity $(14,15)$. Furthermore, in the post-operative setting, it is challenging to determine risk of recurrence and appropriate follow-up for both non-functional and functional tumors. Biomarkers of tumor behavior are therefore urgently needed to personalize pre-operative surgical decision-making and post-operative follow-up intensity (16). Biomarkers may include any 
substance, structure, or process that can reliably be measured in tumor tissue or bodily fluids that can predict the outcome or state of disease (17).

Another key clinical challenge is to determine the origin of neuroendocrine tumor metastases if the primary tumor site cannot be detected by imaging (18). About one out of nine neuroendocrine tumor patients present with metastases of unknown primary origin (19). Similarly, for patients with neuroendocrine tumor syndromes and multiple primary tumors, it is often uncertain which exact tumor specifically metastasized. Knowledge of the site of origin is critical to determine treatment type, follow-up strategies and relevance of prognostic markers (20). A reliable, one-stop test to determine origin on metastases would be a significant improvement to the current diagnostic workup for neuroendocrine tumor metastases of unknown/uncertain primary origin.

To solve these clinical challenges, the gold standard for the pathologist has long been to classify tumors based on tissue or cellular morphology and immunohistochemical characteristics. As reviewed in chapter 2 , this can reliably distinguish several major malignant pancreatic neoplasms, which have a wide variation in prognosis and different treatment strategies. However, neuroendocrine tumors of different behavior and origins show a great deal of overlap in architectural morphology and biomarker expression (18), leaving clinical challenges unsolved. In this thesis, we therefore took a different point of view.

Cell-behavior is the result of a selectively expressed genome, the proteome. The choice of gene expression and translation is no coincidence, but a tightly regulated multilayer process called epigenetic regulation. The mechanism of action includes control of the three-dimensional epigenomic architecture, which determines where the transcription machinery can access the DNA. The epigenomic architecture is built-up in several levels of highly linked covalent modifications or conformational changes. Epigenetic marks include DNA methylation and histone modifications, and are associated with a specific nucleosome occupancy and chromatin architecture. The combined conformational changes result in a specific epigenetic cell morphology that grants selective transcription machinery access to specific regions which maintain cellular differentiation (21). Epigenetic cell or tumor morphology is not only the basis of the morphologic features and protein expression that can be visually assessed by a pathologist, but is also key in regulating all invisible cell-specific processes, making it highly tissue and tumor type specific (22). Currently, epigenetic profiling is used to classify brain tumors with high accuracy and has shown to 
reliably predict origin and type of non-neuroendocrine carcinomas of unknown primary origin $(23,24)$.

Interestingly, many of the genetic mutations in neuroendocrine tumors code for proteins that are involved in chromatin remodeling (including MEN1, ATRX, DAXX, ARID1A, SETD2 (25)) which implies that epigenetic (de)regulation plays an important role in the neuroendocrine tumorigenesis. In fact, several recent studies have used epigenetic cell morphology to predict neuroendocrine tumor behavior or identify molecular subtypes (10, 26-30). For non-functional pancreatic neuroendocrine tumors, whole genome methylation profiling and chromatin immunoprecipitation sequencing studies discovered that many show epigenetic signatures similar to endocrine alpha/glucagon producing cells or beta/ insulin producing cells $(26,30)$. Differential promotor methylation and enhancer activation in alpha and beta cell-like tumors identified the transcription factors aristaless-related homeobox gene (ARX) and pancreatic and duodenal homeobox 1 (PDX1) as surrogate biomarkers of these epigenetic signatures, respectively. These (surrogate) biomarkerbased subtypes held prognostic value and were enriched for specific mutations $(26,30)$. Specifically, ARX-positive/PDX1-negative and ARX-negative/PDX1-negative tumors often metastasized, while ARX-negative/PDX1-positive and ARX-positive/PDX1-positive tumors did not.

This thesis will further explore use of epigenetic neuroendocrine tumor morphology to classify neuroendocrine tumors. The first part focuses on the oncogenic drivers of major duodenopancreatic cell types and their timing in the tumorigenesis. In the second part, epigenetic surrogate biomarkers ARX and PDX1 are used to predict duodenopancreatic neuroendocrine tumor behavior, infer metastatic patterns in patients with MEN1syndrome, and their prognostic value is compared with established prognostic biomarkers. Furthermore, origin specific epigenetic DNA methylation profiles are derived from pulmonary, pancreatic and small intestinal neuroendocrine tumors and are used to predict the origin of neuroendocrine tumors of unknown primary origin. This is followed by a summarizing discussion on how epigenetic neuroendocrine tumor morphology has refined our understanding of neuroendocrine tumorigenesis and behavior, and how this might improve future patient care. 


\section{THESIS OUTLINE}

In part one, we describe the mechanisms of tumorigenesis in duodenopancreatic tumors. In chapter 2, the histopathological morphology, genetic/familial and epigenetic characteristics of malignant pancreatic neoplasms of ductal, acinar or neuroendocrine differentiation are summarized. In chapter 3, we determine the timing of MEN1, ATRX and DAXX loss in pancreatic neuroendocrine tumorigenesis by studying non-syndromic microadenomas (pancreatic neuroendocrine tumors $<5 \mathrm{~mm}$ ), using aberrant immunohistochemical protein expression as surrogate markers of inactivation. In chapter 4, we review the literature for GCHN, and in chapter 5, we investigate the hypothesis that a similar mechanism might contribute to the duodenal gastrin-cell hyperplasia to neoplasia sequence in MEN1syndrome.

In part two, we use epigenetic neuroendocrine tumor morphology or surrogate markers thereof to refine the prognostic and diagnostic classification of neuroendocrine tumors. Reliable cytological determination of novel prognostic biomarkers is critical to their implementation in preoperative surgical decision-making. Therefore, in chapter 6, the accuracy of endoscopic ultrasound guided biopsy to determine ARX and PDX1 expression and other prognostic biomarkers is determined. In chapter 7, we investigate if insulinomas with malignant behavior have different ARX and PDX1 expression and genetic aberrations compared to indolent insulinomas. In chapter 8, we study the independent prognostic value and association of ARX and PDX1 expression versus other clinicopathological biomarkers in a large international multicenter retrospective cohort study of non-functional neuroendocrine tumors of the pancreas. A sub-analysis is performed for the clinically challenging tumors $<2 \mathrm{~cm}$. We also explore the use of ARX and PDX1 expression as markers of pancreatic versus non-pancreatic tumor origin. In chapter 9, we study ARX and PDX1 in non-syndromic unifocal neuroendocrine tumors and matched metastases. After establishing the clonal preservation of ARX and PDX1 during metastases, we inferred relatedness of primary multifocal duodenopancreatic neuroendocrine tumors and metastases in the setting of MEN1-syndrome. Finally, in chapter 10, we use whole genome methylation to detect an origin specific epigenetic neuroendocrine tumor morphology. The use of these epigenetic profiles to predict the site of origin in patients with neuroendocrine tumors of unknown primary origin is evaluated. 


\section{REFERENCES:}

1. Yao JC, Hassan M, Phan A, Dagohoy C, Leary C, Mares JE, et al. One hundred years after "carcinoid": epidemiology of and prognostic factors for neuroendocrine tumors in 35,825 cases in the United States. J Clin Oncol. 2008;26(18):3063-72.

2. Soga J, Tazawa K. Pathologic analysis of carcinoids. Histologic reevaluation of 62 cases. Cancer. 1971;28(4):990-8 3. Lloyd RV, Osamura RY, Klöppel G, Rosai J. WHO Classification of Tumours of Endocrine Organs: International Agency for Research on Cancer; 2017.

4. Man D, Wu J, Shen Z, Zhu X. Prognosis of patients with neuroendocrine tumor: a SEER database analysis. Cancer Manag Res. 2018;10:5629-38.

5. Lubensky IA, Debelenko LV, Zhuang Z, Emmert-Buck MR, Dong Q, Chandrasekharappa S, et al. Allelic deletions on chromosome 11q13 in multiple tumors from individual MEN1 patients. Cancer Res. 1996;56(22):5272-8.

6. Lubensky IA, Pack S, Ault D, Vortmeyer AO, Libutti SK, Choyke PL, et al. Multiple neuroendocrine tumors of the pancreas in von Hippel-Lindau disease patients: histopathological and molecular genetic analysis. Am J Pathol. 1998;153(1):223-31. 7. Yu R, Nissen NN, Dhall D, Heaney AP. Nesidioblastosis and hyperplasia of alpha cells, microglucagonoma, and nonfunctioning islet cell tumor of the pancreas: review of the literature. Pancreas. 2008;36(4):428-31.

8. Henopp T, Anlauf M, Schmitt A, Schlenger R, Zalatnai A, Couvelard A, et al. Glucagon cell adenomatosis: a newly recog nized disease of the endocrine pancreas. J Clin Endocrinol Metab. 2009;94(1):213-7.

9. Scarpa A, Chang DK, Nones K, Corbo V, Patch A-M, Bailey $\mathrm{P}$, et al. Whole-genome landscape of pancreatic neuroendocrine tumours. Nature. 2017;543(7643):65-71.

10. Alcala N, Leblay N, Gabriel AAG, Mangiante L, Hervas $D$, Giffon $T$, et al. Integrative and comparative genomic analyses identify clinically relevant pulmonary carcinoid groups and unveil the supra-carcinoids. Nat Commun. 2019;10(1):3407.

11. Hong X, Qiao S, Li F, Wang W, Jiang R, Wu H, et al. Whole-genome sequencing reveals distinct genetic bases for insulinomas and non-functional pancreatic neuroendocrine tumours: leading to a new classification system. Gut. 2020;69(5):877-87. 12. Francis JM, Kiezun A, Ramos AH, Serra S, Pedamallu CS, Qian ZR, et al. Somatic mutation of CDKN1B in small intestine neuroendocrine tumors. Nat Genet. 2013;45(12):1483-6.

13. Keutgen XM, Schadde E, Pommier RF, Halfdanarson TR, Howe JR, Kebebew E. Metastatic neuroendocrine tumors of the gastrointestinal tract and pancreas: A surgeon's plea to centering attention on the liver. Semin Oncol. 2018;45(4):232-5.

14. Falconi M, Eriksson B, Kaltsas G, Bartsch DK, Capdevila J, Caplin M, et al. ENETS Consensus Guidelines Update for the Management of Patients with Functional Pancreatic Neuroendocrine Tumors and Non-Functional Pancreatic Neuroendocrine Tumors. Neuroendocrinology. 2016;103(2):153-71.

15. Howe JR, Merchant NB, Conrad C, Keutgen XM, Hallet J, Drebin JA, et al. The North American Neuroendocrine Tumor Society Consensus Paper on the Surgical Management of Pancreatic Neuroendocrine Tumors. Pancreas. 2020;49(1):1-33. 16. Jensen RT, Bodei L, Capdevila J, Couvelard A, Falconi
M, Glasberg S, et al. Unmet Needs in Functional and Nonfunctional Pancreatic Neuroendocrine Neoplasms. Neuroendocrinology. 2019;108(1):26-36.

17. Strimbu K, Tavel JA. What are biomarkers? Curr Opin HIV AIDS. 2010;5(6):463-6.

18. Bellizzi AM. Immunohistochemistry in the diagnosis and classification of neuroendocrine neoplasms: what can brown do for you? Hum Pathol. 2020;96:8-33.

19. Scoazec JY, Couvelard A, Monges G, Guyetant S,

Bisot-Locard S, Parot $X$, et al. Professional Practices and Diagnostic Issues in Neuroendocrine Tumour Pathology: Results of a Prospective One-Year Survey among French Pathologists (the PRONET Study). Neuroendocrinology. 2017;105(1):67-76.

20. Herrera-Martinez AD, Hofland LJ, Galvez Moreno MA Castano JP, de Herder WW, Feelders RA. Neuroendocrine neoplasms: current and potential diagnostic, predictive and prognostic markers. Endocr Relat Cancer. 2019;26(3):R157-R79.

21. Lee DS, Luo C, Zhou J, Chandran S, Rivkin A, Bartlett $A$, et al. Simultaneous profiling of 3D genome structure and DNA methylation in single human cells. Nat Methods. 2019;16(10):9991006.

22. Lokk K, Modhukur V, Rajashekar B, Martens K, Magi R, Kolde $\mathrm{R}$, et al. DNA methylome profiling of human tissues identifies global and tissue-specific methylation patterns. Genome Biol. 2014;15(4):r54.

23. Moran S, Martinez-Cardus A, Sayols S, Musulen E, Balana C, Estival-Gonzalez A, et al. Epigenetic profiling to classify cancer of unknown primary: a multicentre, retrospective analysis. Lancet Oncol. 2016;17(10):1386-95.

24. Capper D, Jones DTW, Sill M, Hovestadt V, Schrimpf $D$, Sturm D, et al. DNA methylation-based classification of central nervous system tumours. Nature. 2018;555(7697):469-74.

25. Pipinikas CP, Berner AM, Sposito T, Thirlwell C. The evolving (epi)genetic landscape of pancreatic neuroendocrine tumours. Endocr Relat Cancer. 2019;26(9):R519-R44.

26. Chan CS, Laddha SV, Lewis PW, Koletsky MS, Robzyk K, Da Silva E, et al. ATRX, DAXX or MEN1 mutant pancreatic neuroendocrine tumors are a distinct alpha-cell signature subgroup. Nat Commun. 2018;9(1):4158.

27. Karpathakis A, Dibra H, Pipinikas C, Feber A, Morris T, Francis J, et al. Prognostic Impact of Novel Molecular Subtypes of Small Intestinal Neuroendocrine Tumor. Clin Cancer Res. 2016;22(1):250-8.

28. Laddha SV, da Silva EM, Robzyk K, Untch BR, Ke $\mathrm{H}$, Rekhtman N, et al. Integrative Genomic Characterization Identifies Molecular Subtypes of Lung Carcinoids. Cancer Res. 2019;79(17):4339-47.

29. Tirosh A, Killian JK, Petersen D, Zhu YJ, Walker RL, Blau JE, et al. Distinct DNA Methylation Signatures in Neuroendocrine Tumors Specific for Primary Site and Inherited Predisposition. J Clin Endocrinol Metab. 2020;105(10).

30. Cejas P, Drier Y, Dreijerink KMA, Brosens LAA, Deshpande $V$, Epstein $C B$, et al. Enhancer signatures stratify and predict outcomes of non-functional pancreatic neuroendocrine tumors.

Nat Med. 2019;25(8):1260-5. 


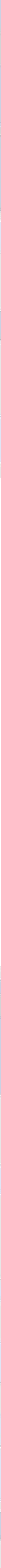




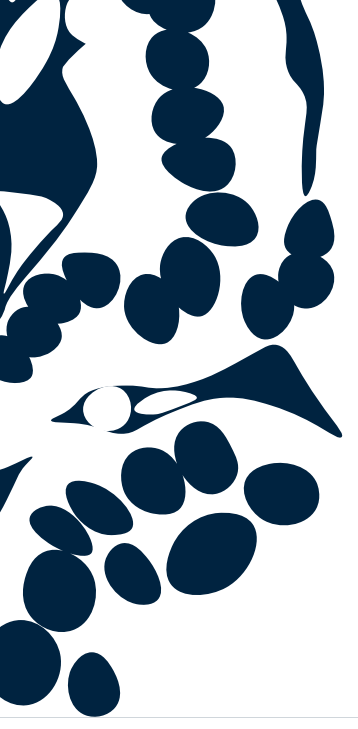

CELL-DIFFERENTIATION SPECIFIC DUODENOPANCREATIC TUMORIGENIC PATHWAYS
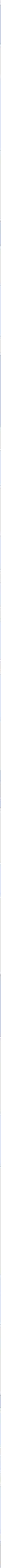

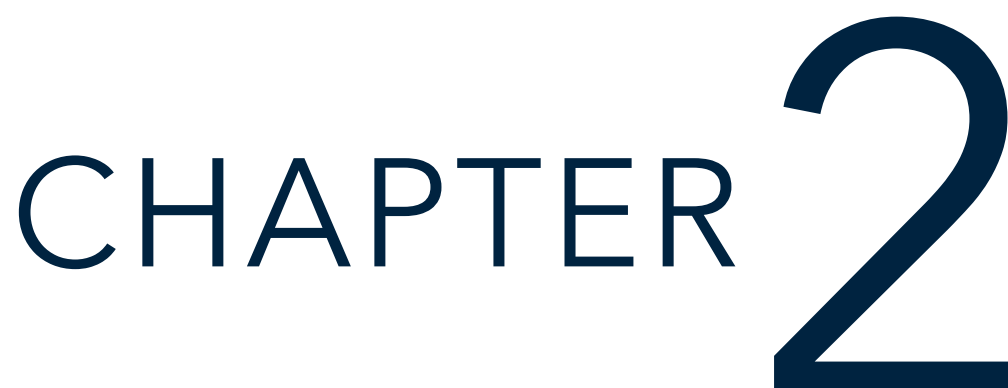

Surgical and Molecular Pathology of Pancreatic Neoplasms 


\section{ABSTRACT}

Histologic characteristics have proven to be very useful for classifying different types of tumors of the pancreas. As a result, the major tumor types in the pancreas have long been classified based on their microscopic appearance. Recent advances in whole exome sequencing, gene expression profiling, and knowledge of tumorigenic pathways have deepened our understanding of the underlying biology of pancreatic neoplasia. These advances have not only confirmed the traditional histologic classification system, but also opened new doors to early diagnosis and targeted treatment. Here we review the pathology, genetic and epigenetic alterations and potential treatment targets of the five major malignant pancreatic tumors - pancreatic ductal adenocarcinoma, pancreatic neuroendocrine tumor, solid-pseudopapillary neoplasm, acinar cell carcinoma and pancreatoblastoma. 


\section{INTRODUCTION}

Malignant neoplasms of the pancreas are currently classified based on the cellular direction of differentiation (ductal, acinar or neuroendocrine) of the neoplastic cells, combined with the macroscopic appearance (solid or cystic) of the tumors. Pancreatic ductal adenocarcinoma comprises about $90 \%$ of all malignant pancreatic neoplasms. Of all other malignant pancreatic neoplasms (pancreatic neuroendocrine tumors, solid-pseudopapillary neoplasm, acinar cell carcinoma and pancreatoblastoma), neuroendocrine tumors are the most common, comprising approximately $5 \%$ of malignant pancreatic tumors (Table 1).

Recent genetic and epigenetic characterization of these histologically distinct pancreatic tumors has increased our understanding of common genetic signatures, and has also identified tumor specific genetic alterations (Table 2). In addition to serving as diagnostic tools, some genetic alterations can be exploited as targets for therapy, opening avenues for new treatments. In this review, histology, genetics and epigenetics of malignant pancreatic tumors and potential targets for treatment are discussed.

\section{PANCREATIC DUCTAL ADENOCARCINOMA}

Infiltrating ductal adenocarcinoma, also known as pancreatic ductal adenocarcinoma (PDAC), accounts for $90 \%$ of all malignant pancreatic neoplasms and occurs at a mean age of 66 years (1). PDAC has a very poor prognosis with an overall 5-year survival of only $7 \%$ (2). At diagnosis, the majority of patients are inoperable due to locally advanced or metastatic disease. The median survival for patients with metastatic disease is less than a year (3). Moreover, by the year 2030 pancreatic cancer is predicted to become the second leading cause of cancer-related death in the U.S. (4). In view of the increasing incidence and the virtually unchanged poor prognosis of PDAC both new therapies for established pancreatic cancer as well as methods for prevention and early detection are desperately needed.

\section{Gross and microscopic findings}

PDACs are characteristically firm, ill-defined white-yellow masses (Figure 1A). The pancreatic parenchyma upstream from PDACs is usually atrophic and the main pancreatic duct can be dilated. Microscopically, PDAC is composed of haphazardly arranged infiltrating glandular and ductal structures typically surrounded by abundant desmoplastic stroma. The cells have eosinophilic to clear cytoplasm and usually enlarged pleomorphic nuclei. Poorly differentiated ductal adenocarcinomas have more irregular and smaller glands and 


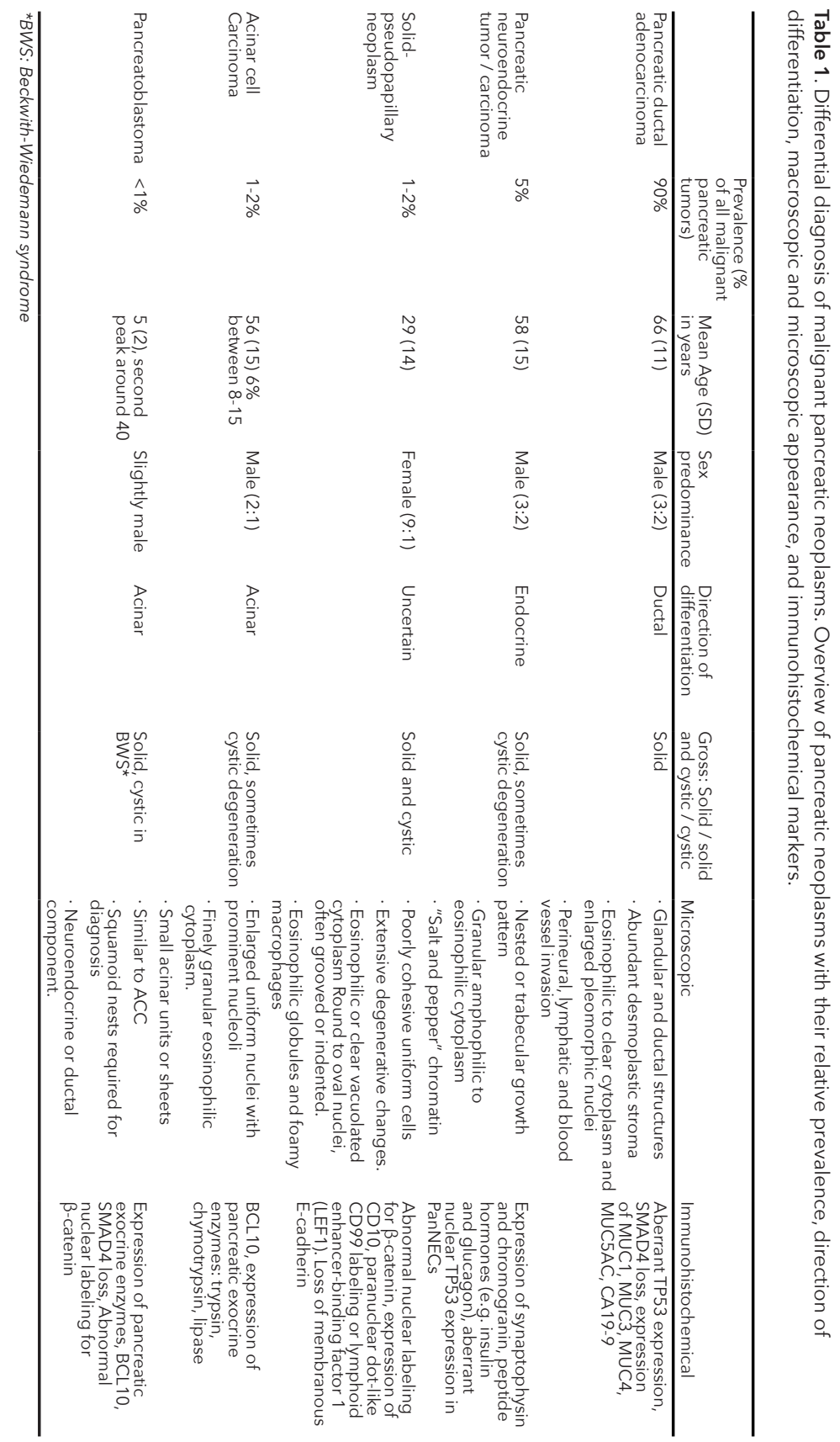


Table 2. Overview of pancreatic neoplasms with their key genetic alterations and several epigenetic alterations discussed in this review.

\begin{tabular}{|c|c|c|c|c|}
\hline & $\begin{array}{l}\text { Average number } \\
\text { of somatic } \\
\text { mutations }\end{array}$ & Major genes involved & Methylation & $\begin{array}{l}\text { MiRNA tumor expression } \\
\text { compared to normal } \\
\text { pancreatic tissue }\end{array}$ \\
\hline \multirow[t]{2}{*}{$\begin{array}{l}\text { Pancreatic ductal } \\
\text { adenocarcinoma }\end{array}$} & \multirow[t]{2}{*}{$20-80$} & \multirow{2}{*}{$\begin{array}{l}\text { KRAS, CDNK2A, } \\
\text { SMAD44, TP53, MLL3, } \\
\text { TGFBR2, ATM, ARID1A, } \\
\text { ROBO2, KDM6A }\end{array}$} & \multirow[t]{2}{*}{$\begin{array}{l}\text { Loss of function through promotor } \\
\text { hypermethylation: } C D N K 2 A, h M L H 1\end{array}$} & $\begin{array}{l}\text { Upregulation: miR-21, 23a, } \\
31,100,143,155 \text {, and } 221\end{array}$ \\
\hline & & & & $\begin{array}{l}\text { Downregulation: miR-148a, } \\
217 \text { and } 375\end{array}$ \\
\hline \multirow{2}{*}{$\begin{array}{l}\text { Pancreatic } \\
\text { Neuroendocrine } \\
\text { tumor }\end{array}$} & \multirow[t]{2}{*}{16} & \multirow[t]{2}{*}{$\begin{array}{l}\text { MEN1, ATRX, DAXX, } \\
\text { TSC2, PTEN, Rb, TP53\# }\end{array}$} & \multirow{2}{*}{$\begin{array}{l}\text { Hypomethylation of LINE1 and hyper- } \\
\text { methylation of RASSF1A promoting the } \\
\text { accumulation of } \beta \text {-catenin }\end{array}$} & $\begin{array}{l}\text { Upregulation: miR-193b, } \\
103 \text { and } 107\end{array}$ \\
\hline & & & & Downregulation: miR-155 \\
\hline $\begin{array}{l}\text { Solid- } \\
\text { pseudopapillary } \\
\text { neoplasm }\end{array}$ & 3 & CTNNB1 & u & $\begin{array}{l}\text { MiRNAs possibly upregu- } \\
\text { lating the Wnt, Hedgehog, } \\
\text { and Androgen receptor } \\
\text { pathway }\end{array}$ \\
\hline \multirow[t]{2}{*}{$\begin{array}{l}\text { Acinar cell } \\
\text { carcinoma }\end{array}$} & \multirow[t]{2}{*}{131} & \multirow{2}{*}{$\begin{array}{l}\text { SMAD4, JAK1, BRAF, } \\
\text { RB1,TP53, APC, } \\
\text { ARID1A, GNAS, MLL3, } \\
\text { PTEN }\end{array}$} & \multirow[t]{2}{*}{$\begin{array}{l}\text { Hypermethylation of RASSF1, MLH1 } \\
\text { and APC }\end{array}$} & $\begin{array}{l}\text { Upregulation: miR-17, 20, } \\
21,92-1,103,107\end{array}$ \\
\hline & & & & Downregulation: miR-155 \\
\hline Pancreatoblastoma & 18 & $\begin{array}{l}\text { Loss of chromosome } \\
11 p, \text { CTNNB1 }\end{array}$ & Hypermethylation of RASSF1A & u \\
\hline
\end{tabular}

u, unknown, \# MEN1, ATRX, DAXX, TSC2 and PTEN mutations are found in well-differentiated PanNET but not in PanNEC. ${ }^{*}$ Rb and TP53 mutations are present in PanNEC, but not in well-differentiated PanNET
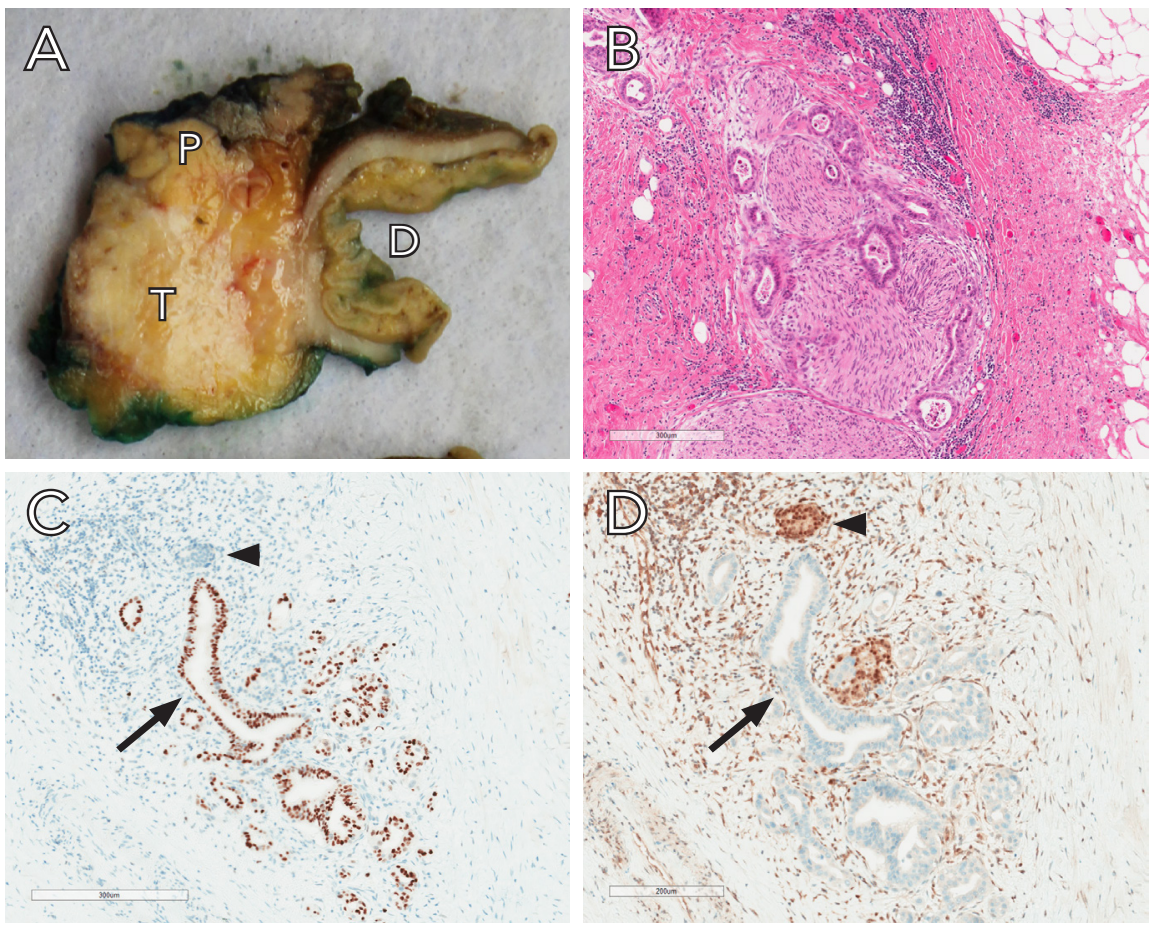

Figure 1. A, macroscopic appearance of a pancreatic ductal adenocarcinoma showing a poorly demarcated firm white tumor in the pancreatic parenchyma (T, Tumor; $P$, pancreatic parenchyma; $D$, duodenum). $B$, perineural invasion of a pancreatic ductal adenocarcinoma. C, positive TP53 immunohistochemistry in pancreatic ductal adenocarcinoma indicative of TP53 gene mutation. Arrow, malignant ductal structure; arrowhead, normal pancreatic duct. D, loss of SMAD4 immunohistochemistry in pancreatic ductal adenocarcinoma indicating mutation of the SMAD4 gene. Arrow, malignant ductal structure; arrowhead, normal pancreatic duct. 

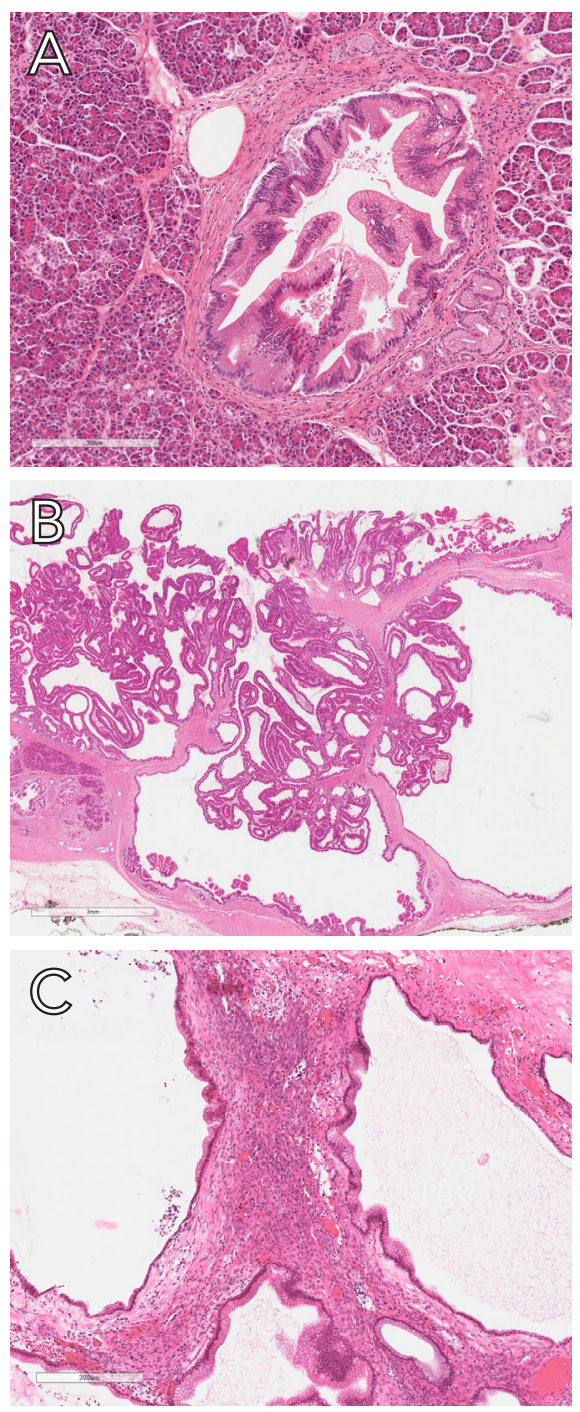

Figure 2. A, low-grade pancreatic intraepithelial neoplasia (PanIN) showing micro-papillary epithelium with mild to moderate cytological atypia. B, intraductal papillary mucinous neoplasm (IPMN), gastric-foveolar type with low-grade dysplasia. C, mucinous cystic neoplasm (MCN) showing gastric-foveolar type epithelium with low-grade dysplasia, surrounded by ovarian-type stroma. 


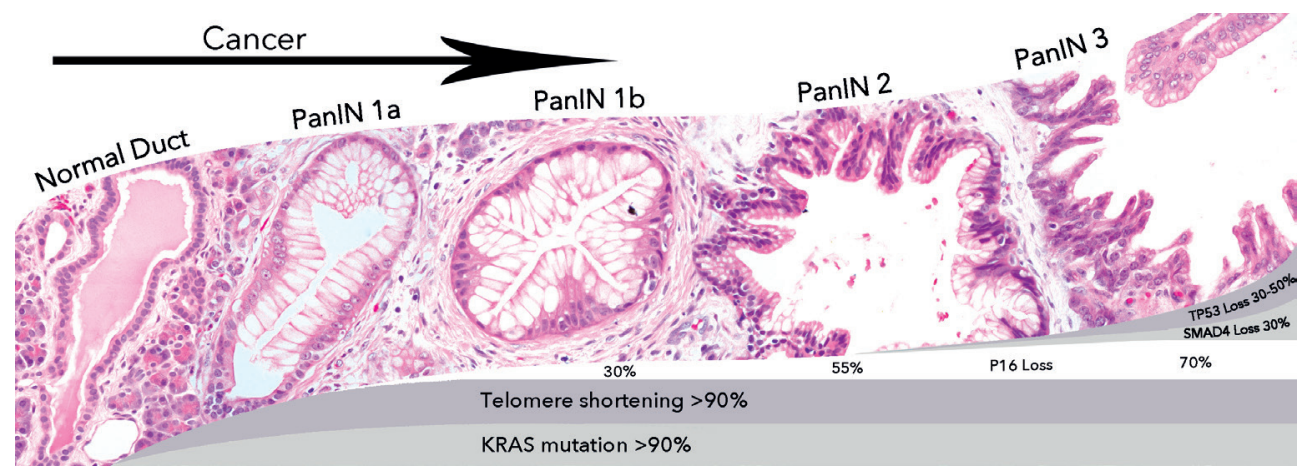

Figure 3. Pancreatic cancer develops from the well-defined precursor lesions pancreatic intraepithelial neoplasia, intraductal papillary mucinous neoplasm and mucinous cystic neoplasm. The PanIN progression model shown here shows that accumulation of genetic and epigenetic alterations drives neoplastic progression in these precursor lesions from low-grade dysplasia (PanIN 1 and PanIN 2) to high-grade dysplasia (PanIN 3) to eventually an invasive pancreatic adenocarcinoma.

significant pleomorphism. Perineural, lymphatic and blood vessel invasion are frequently present (Figure 1B). The neoplastic cells in areas of venous invasion can be so welldifferentiated that they mimic non-invasive precursor lesions (pancreatic intraepithelial neoplasia). Immunohistochemically, there is no definite marker to distinguish PDAC from non-neoplastic ductal structures, although aberrant TP53 expression or SMAD4 loss support the diagnosis of PDAC over reactive glands (Figure 1C and D) $(5,6)$. Several types of mucin (MUC1, MUC3, MUC4, MUC5AC) and glycoprotein tumor antigens such as CA199 can be expressed in PDAC (7-9). The main microscopic differential diagnosis consists of PDAC precursor lesions, other malignant pancreatic tumors (Table 1), pancreatitis and adenocarcinoma metastasis.

Table 3. Overview of germline genetic alterations with well-defined pancreatic cancer risk and genes that have been associated with familial PDAC.

\begin{tabular}{ll}
\hline Gene (syndrome) & RR (Cumulative lifetime risk (\%) by age 70) \\
\hline STK11/LKB1 (Peutz-Jeghers syndrome) & $132(36)$ \\
PRSS1/SPINK1 (hereditary pancreatitis) & $50-80(40)$ \\
CDKN2A (FAMMM) & $13-47(17)$ \\
BRCA1/BRCA2 (HBOC) & $3.5-10(3-8)$ \\
MLH1, MSH2, MSH6, PMS2 (Lynch syndrome) & $8.6(<5)$ \\
CFTR (cystic fibrosis) & $5(<5)$ \\
FDR with PC & $2-3(2)$ \\
FDRs with PC & $6(8-12)$ \\
Possible role in FPC: ATM, TET2, DNMT3A, POLN, POLQ, ASXL1, PALB2, FAN- & Unknown \\
CG, BUB1B, ESCO2, FANCC, FANCM, MSH4, RAD54L & \\
\hline RR, relative risk; FDR, first degree relative; FAMMM, familial atypical multiple mole melanoma; HBOC, hereditary breast and ovarian \\
cancer syndrome; FAP. familial adenomatous polyposis; PC, pancreatic Cancer; FPC, familial pancreatic cancer. Adapted from Ghiorzo \\
et al. and Roberts et al. (12,151)
\end{tabular}


PDAC develops from precursor lesions that can be either microscopic (pancreatic intraductal neoplasia, PanIN) or macroscopic cystic precursor lesions (intraductal papillary mucinous neoplasm, IPMN; mucinous cystic neoplasm, MCN) (Figure 2). IPMN and MCN are often found as incidental finding on imaging. PanIN arises in microscopic ducts; IPMN arises within the main- or branch-ducts. $\mathrm{MCN}$ usually does not communicate with the ductal system. Microscopically, all precursors show flat or papillary mucin-producing neoplastic epithelium, with varying degrees of dysplasia and directions of differentiation. Stepwise accumulation of (epi)genetic alterations drives neoplastic progression and eventually development of malignant invasive carcinoma, analogous to the PanIN progression model as depicted in Figure 3 and discussed below $(10,11)$.

\section{Genetic Signature: familial PDAC}

Approximately $10 \%$ of pancreatic cancers appear to have an inherited component. Overall, sporadic and familial PDAC share the same driver mutations (KRAS, CDKN2A, TP53 and $S M A D 4)(12)$, but some of these cases are caused by inherited germline genetic alterations in genes that significantly increase the risk of pancreatic cancer (Table 3 ). These genes include BRCA2, BRCA1, PALB2, p16/CDKN2A, ATM, STK11, PRSS1, and the DNA mismatch repair genes (such as $M L H 1$ and $M S H 2$ ) (13-17). In addition, a number of other candidate genes, such as BUB1B, CPA1, FANCC and FANCG, have been described (12). These germline alterations are critical to understand because the risk is significant and at-risk patients can be enrolled in screening and early detection protocols for pancreatic and extra-pancreatic tumors (18). In addition, some patients with specific genetic alterations can be prioritized for specific therapies. For example, some tumors characterized by microsatellite instability due to a DNA mismatch repair gene defect are exquisitely responsive to immunotherapies, and some tumors with BRCA or PALB2 gene mutation are sensitive to poly ADP ribose polymerase (PARP)-inhibitors (19-21).

In addition to these low prevalence but high penetrance genes, there are a number of more common lower penetrance genes that increase the risk of pancreatic cancer only slightly. A number of these, including $A B O$ blood group type, have been identified in genome wide association studies (GWAS) (22-24).

\section{Genetic Signature: sporadic PDAC}

The somatic alterations present in PDAC are now well characterized thanks to several large whole-exome and whole-genome sequencing studies $(21,25-27)$. On average PDACs 
have 50-80 exomic non-silent mutations (21, 25-27). In addition, extensive larger structural variations including intra-chromosomal rearrangements, deletions and amplifications are common in PDAC $(21,28)$.

Point mutation of the oncogene KRAS is seen in almost all early pancreatic cancer precursor lesions and in PDACs. Subsequent mutations that drive neoplastic progression in PanIN lesions are usually in the tumor suppressor genes CDKN2A, TP53 and SMAD4 (Figure 3) $(21,25,26)$. Further accumulation of genetic and epigenetic alterations drives neoplastic progression in these precursor lesions, eventually leading to an invasive pancreatic adenocarcinoma (10). Less commonly mutated genes in PDAC include MLL3, TGFBR2, ATM, ARID1A, ROBO2 and KDM6A (21, 25-27). Of note, mutations in chromatin-regulating genes (MLL, MLL2, MLL3 and ARID1A) have been associated with improved survival, and loss of SMAD4 with poorer survival $(29,30)$. Many mutations found by whole exome sequencing are reported in a very low percentage of tumors, and therefor categorized as passengers in tumorigenesis. Of note the recently proposed "mini driver" model hypothesizes that several passengers might have relatively weak tumor-promoting effects but together might substitute for a major-driver (31). More research is needed to address the exact role of most of these less prevalent mutations in tumorigenesis.

Importantly - despite the diversity of genes targeted - the genetic alterations in PDAC appear to selectively target core signaling pathways, including Wnt/Notch signaling, TGF- $\beta$ signaling, and DNA damage control (26). Despite the genetic heterogeneity of PDAC, targeting one or more of these pathways may be more effective than targeting a specific genetic alteration. For example, Waddell et al. recently correlated deleterious mutations in BRCA1 and BRCA2 with unstable genetic signatures (>200 structural variations). In their study, 4 out of 5 patients with defective DNA damage control responded to treatment with a platinum containing regimen. Also PARP inhibitors have been reported to be effective in BRCA mutated tumors (21). These findings illustrate how knowledge of rare mutations in known pathways can be used to guide treatment. A number of clinical trials targeting specific pathways and mutations are being conducted on patients with PDAC and other human cancers. Potential targets for therapeutic intervention are seen in over a third of PDACs (up to 97\% when trials related to KRAS and TP53 are included) (29). Future personalized treatment might thus drastically change outcome of this disease.

Studies of the clonal evolution of genetic changes in pancreatic cancer and metastases 
by Yachida et al. suggest that it takes almost 12 years from the initiating mutation in the pancreas until development of an invasive PDAC (32). This suggests a wide window of opportunity for the early detection of pancreatic cancer. The genetic alterations present in pancreatic cancer and its non-invasive precursors can be shed into the blood and into the pancreatic duct system. This suggests the possibility of gene-based early detection tests. Indeed, mutant KRAS shed from invasive pancreatic cancer can be detected in the plasma, and mutations present in non-invasive cystic precursor lesions, such as IPMNs and MCNs can be detected in cyst fluid aspirated at the time of endoscopic ultrasound (EUS), as well as in secretin stimulated pancreatic juice collected from the duodenum $(33,34)$.

\section{Epigenetic alterations}

A number of genes are aberrantly methylated in pancreatic cancer (35-41). For example, integrated methylation and gene-expression meta-analysis have identified a number of genes (MUC4, SERPINB5, CLDN4, SFN, TFF1, S100P, S100A4, MMP1, MMP7, MSLN, PSCA, ID1, MST1R, NBL1, PHLDA2, PLAT, PLAUR, IL8, SPP1, ARHGDIB, NQO1, and ITGB4) that are significantly upregulated in PDAC, likely caused by promoter hypomethylation $(36,42,43)$. Some of the genes targeted by changes in methylation are clearly cancer-causing, such as the well-known tumor suppressor gene CDNK2A and the DNA repair gene $h M L H 1$, which show loss of function through promoter hypermethylation silencing $(40,44-46)$.

These epigenetic changes are not only functionally important, but can also be used as markers of disease and early detection. For example, DNA methylation alterations in the pancreatic juice are a possible approach to the diagnosis of pancreatic cancer (47).

\section{MicroRNA}

Post-transcriptional regulation or silencing of gene expression occurs mostly by noncoding RNAs. The most studied non-coding RNAs are microRNAs (miRs), which are small single stranded RNA molecules that regulate mRNA by full or partial complementarity. Deregulated miRs can give information on transcriptional regulation and may serve as biomarkers for survival and early detection (48-50).

Recently a large meta-analysis looked at the miR expression profiles of 538 PDACs. A statistically significant miR meta-signature with upregulation of miR-21, 23a, 31, 100, 143, 155, and 221 and downregulation of miR-148a, 217 and 375 was found in PDAC. Furthermore, in a cohort of 70 patients, the high expression of miR-21, miR-31 and the low expression of miR-375 in their PDACs was found to be an independent prognostic marker 
for poor overall-survival (50). Interestingly, in stool from patients with PDAC, significantly higher miR-21 and miR-155 and lower miR-216 levels have been found compared to normal controls (51). Other studies with "disease free survival" and "overall survival" as outcome measures also found an important role for high levels of miR-21 in predicting prognosis, along with high miR-155, high miR-203, and low miR-34a (49).

MiR-21 is thus an important candidate for diagnostic and prognostic purposes, although it cannot be used to differentiate between PDAC and precursor lesions such as intraductal papillary mucinous neoplasms (IPMN) or malignancy in other organs $(52,53)$. MiR-21 has approximately 180 target messenger RNAs (mRNA) (54). Interestingly several of these targets are tumor suppressors and negative regulators of the Ras/MEK/ERK pathway. An in vivo study with a murine non-small cell lung carcinoma model confirmed upregulation of miR-21 with RAS activation, and downregulation of several negative RAS regulators and tumor suppressors including SPRY1, SPRY2, BTG2, and PDCD4 (54). In vitro studies have reported several other miR-21 affected tumor suppressor mRNAs, including PTEN (55). Deletion of miR-21 has also been shown to repress tumor formation in KRAS mutated mice and makes in vitro cells more sensitive for chemotherapy, possibly by repression of the AKT pathway through p85a inhibition (56). MiR-21 may thus be potentially interesting as pharmacological target as well.

Research on miRs is booming, and many recent studies have found other and new miRs not reported in the meta-reviews, also to be excellent prognostic markers for PDAC (5760). Other forms of non-coding RNA like long non-coding RNA (IncRNA), small nucleolarderived RNA (sdRNA) and piwi-interacting RNA (piRNA) are also differentially altered in PDAC (61).

\section{Changes in gene expression}

Several studies have tried to classify PDAC into clinically meaningful subgroups based on gene expression profiles. Collisson et al. clustered 3 distinct subtypes of PDAC (classical, quasimesenchymal and exocrine-like) with different responses to treatment and different patient prognosis (62). Recently Moffitt et al. used blinded digital separation of PDAC gene expression microarray data to cluster primary carcinoma, metastasis, and normal samples (63). They found that the groups described by Collisson et al. did not hold predictive power in their samples. Instead they identified two tumor subtypes: "classical" which had great overlap with the classical group of Collison et al., and basal-like which had a worse outcome 
and was molecularly similar to basal tumors in the bladder. Furthermore, as they could digitally separate tumor and stromal expression, they defined "normal" and "activated" stromal subtypes, which they reported to be independent prognostic factors (63). Currently, there is no well-established clinically meaningful subclassification of PDAC.

Differentially upregulated genes by mRNA can result in upregulation of proteins, which - just like DNA, miRNA and mRNA - can be used as potential diagnostic biomarkers of malignancy in pancreatic juice and blood (64). Furthermore, specific mutated proteins such as mutant Ras can be distinguished from wild-type Ras by mass spectrometry in tissue and pancreatic juice, which might be even more useful for early diagnosis of PDAC and its precursors (65). Other highly expressed proteins including mesothelin are potentially targetable with immunotherapy (66). Mutant proteins can also give rise to aberrant epitopes on tumor $\mathrm{MHC}$ receptors, which then can specifically be targeted by adoptive T-cell therapy as elegantly demonstrated in other human cancers (67).

\section{Stroma and the tumor microenvironment}

In addition to genetic alterations, the tumor microenvironment and changes in epigenetic regulation play important roles in promoting or suppressing PDAC growth $(68,69)$ and stromal expression profile has shown prognostic significance (63). Also, by overexpression of hyaluronic acid and collagens, the extracellular matrix can cause a high interstitial fluid pressure, causing compression of blood vessels and therefor hindering passive transport processes of chemotherapeutics. Targeting these stromal factors might improve therapeutic response (70).

PDAC and its microenvironment are also marked by distinct immune cell populations along its path of tumorigenesis, creating an immunosuppressive environment that shields tumor cells from detection and renders them resistant to immune-based therapies. Regulatory T-cells (T-reg) seem to play a role from the earliest stage of precursor disease potentially undermining anti-tumor effector T-cell activity; high intratumor T-reg/CD4+ T-cell ratio is a prognostic factor for worse survival. Therapeutically targeting of T-regs in malignancy is currently under investigation (70).

\section{PANCREATIC NEUROENDOCRINE TUMORS}

Pancreatic neuroendocrine tumors (PanNET) are the second most common malignant tumor of the pancreas (6). PanNETs occur mostly in elderly patients, with a mean age of 58 years (71). Although prognosis of PanNET is better than PDAC, it is still poor with an 
Table 4. Mutations in pancreatic MEN1 syndrome associated microadenomas and PanNETs, sporadic PanNETs and PanNECs

\begin{tabular}{|c|c|c|c|c|c|c|}
\hline \multirow[t]{2}{*}{ Neoplasm } & \multicolumn{6}{|l|}{ Mutations } \\
\hline & MEN1 & $A T R X / D A X X$ & $\begin{array}{l}\text { mTOR } \\
\text { pathway }\end{array}$ & KRAS & TP53 & $R B 1$ \\
\hline MEN1 syndrome microadenomas & Up to $100 \%$ & $0 \%$ & $\mathrm{u}$ & $\mathrm{u}$ & $\mathrm{u}$ & $\mathrm{u}$ \\
\hline MEN-1 syndrome tumors & Up to $100 \%$ & $6 \%$ & u & u & u & u \\
\hline G1/G2 pancreatic neuroendocrine tumors & $45 \%$ & $45 \%$ & $15 \%$ & $0 \%$ & $0 \%$ & $0 \%$ \\
\hline G3 pancreatic neuroendocrine carcinomas & $\mathrm{u}$ & $0 \%$ & $\mathrm{u}$ & 30 & $60 \%$ & $70 \%$ \\
\hline
\end{tabular}
u, unknown

average overall 5 year survival of only $42 \%$ (72). Functional PanNETs are well known for their classic clinical presentations including Whipple's triad (insulinoma) and Zollinger-Ellison syndrome (gastrinoma), in which hypersecretion of pancreatic or non-pancreatic hormones have systemic effects. When no systemic effects of hormone production are seen, PanNETs are by definition classified as non-functional (5).

A number of TNM classification systems with prognostic value for PanNET patients have been developed by the WHO2010 [World Health Organization], ENETs [European Neuroendocrine Tumor Society] and AJCC [American Joint Committee on Cancer] (73). Although it is at present not completely established which system should be preferred, a recent study suggests that the ENETS TNM classification was superior to the AJCC/ WHO2010 classification/grading System and more accurate (74).

\section{Gross and microscopic findings}

PanNETs are usually soft, sometimes red or white, well-demarcated lesions (Figure 4A). Microscopically the neoplastic cells have a nested or trabecular growth pattern. At higher magnification, the neoplastic cells have a distinct neuroendocrine morphology, with a granular amphophilic to eosinophilic cytoplasm and the typical coarsely clumped "salt and pepper" chromatin (Figure 4B). The mitotic rate and percentage of Ki67 positive cells are used for grading. The well-differentiated PanNETs can be either grade 1 ( $<2$ mitoses per 10 HPF; Ki-67 labeling index <2\%) or grade 2, (2-20 mitoses per 10 HPF; Ki-67 labeling index $3-20 \%)$. If mitotic count is $>20$ mitoses per $10 \mathrm{HPF}$ or $\mathrm{Ki}-67$ index is $>20 \%$, the neoplasm is classified as a grade 3 neuroendocrine tumor or neuroendocrine carcinoma (PanNEC). Histologically PanNECs can have one of two appearances. Those with a Ki-67<50\% can look similar to the well-differentiated PanNETs, only they have a high proliferation rate (75). This group is somewhat more aggressive than grade 2 PanNETs but not as rapidly progressive as the PanNECs with a Ki67 > 50\%. PanNECs with a very high proliferation rate 

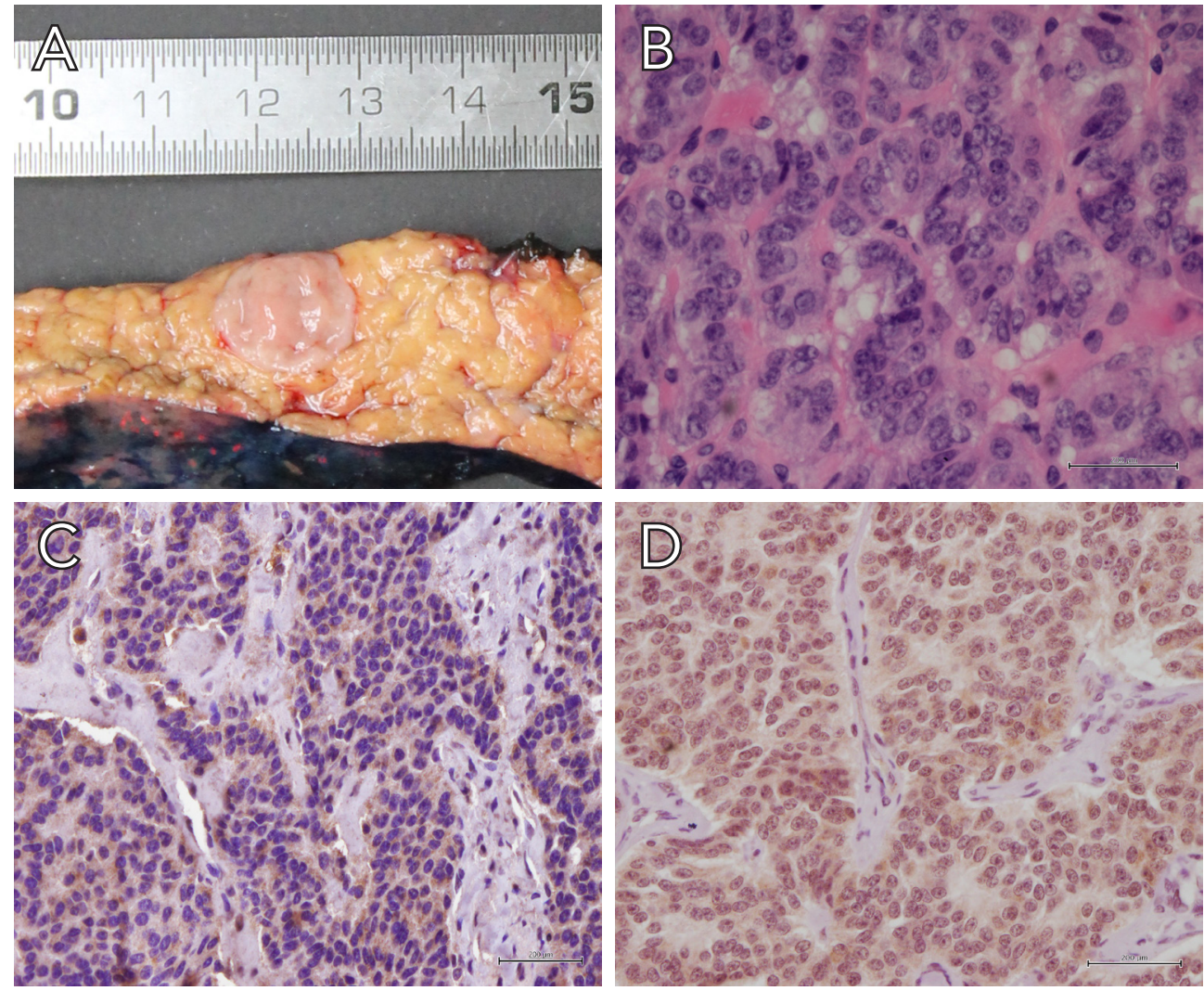

Figure 4. A, macroscopic appearance of pancreatic neuroendocrine tumor showing a well-demarcated pinkish tumor surrounded by normal pancreatic parenchyma. B, pancreatic neuroendocrine tumor, detail showing typical salt and pepper chromatin. C, loss of Menin expression in pancreatic neuroendocrine tumor indicative of MEN1 gene inactivation. D, retained Menin expression in pancreatic neuroendocrine tumor with a wildtype MEN1 gene

(>50\%) can have a small cell carcinoma or large cell carcinoma appearance with markedly pleomorphic typical neuroendocrine cells that are tightly packed in nests or form diffuse irregular sheets (5). Necrosis is often seen.

Immunohistochemical expression of neuroendocrine markers synaptophysin and chromogranin A is seen in the majority of PanNETs (76), and peptide hormones (e.g. insulin and glucagon) can also confirm neuroendocrine differentiation. Large cell PanNECs typically express neuroendocrine markers, but small cell PanNECs may not. Both large and small cell PanNECs are typically negative for peptide hormones (77). Aberrant nuclear TP53 expression is commonly found in PanNECs but is never seen in PanNETs (78). The 
main microscopic differential diagnosis consists of acinar cell carcinoma (Figure 7), pancreatoblastoma, mixed neuroendocrine tumors, and dedifferentiated PDAC (Table 1).

\section{Genetic Signature: Familial PanNET}

The vast majority (90\%) of PanNETs occur sporadically, but some occur in the setting of associated familial syndromes including multiple endocrine neoplasia type 1 (MEN1), von Hippel-Lindau syndrome (VHL), neurofibromatosis type 1 (NF1), tuberous sclerosis complex (TSC) and the recently discovered glucagon cell adenomatosis (GCA) (5, 79). Studies of PanNETs occurring in patients with an underlying genetic predisposition have provided important insight into the genes involved in tumorigenesis of PanNETs. Tumorigenesis in these syndromes follow a hyperplasia-neoplasia sequence in which hyperplastic nodules transform over time into frank neoplasms (79-81). It is assumed that sporadic PanNETs develop through a similar hyperplasia-neoplasia sequence. PanNECs are not associated with germline syndromes and are believed to follow a different pathway of tumorigenesis (78).

\section{Genetic Signature: Sporadic PanNET}

Whole exome and targeted sequencing of well differentiated PanNETs (grade 1 and 2) of patients without a familial syndrome showed an average of only 16 nonsynonymous mutations per tumor (82). Somatic mutations of the MEN1 gene were found in $45 \%$ of these sporadic PanNETs (82). Others have previously reported loss of heterozygosity at the MEN1 locus in $20-45 \%$ of sporadic PanNETs $(83,84)$. In addition to prevalent somatic MEN1 mutations, $45 \%$ of sporadic PanNETs harbored somatic inactivating mutations in ATRX or DAXX, and $15 \%$ had mutations in mTOR pathway genes (in which TSC1/2 functions) (82). Remarkably, the alternative lengthening of telomeres (ALT) phenotype, a mechanism of telomerase independent telomere maintenance to overcome cell death, was found to correlated perfectly with loss of ATRX and DAXX (85-87). Moreover, many gains and losses have been reported in sporadic PanNETs $(88,89)$. VHL is deleted in $18 \%$ of sporadic PanNET, and recently allelic loss of PHLDA3 - a regulator of the mTOR pathway - was found in $70 \%$ of sporadic PanNETs $(90,91)$.

The genetic alterations in well-differentiated PanNETs (grades 1 and 2) have been compared to those in PanNEC (grade 3). Yachida et al. found that small and large cell PanNECs are genetically similar, but distinct from PanNETs (78). In PanNECs, activating KRAS mutations (2 of 7) and inactivating mutations in TP53 (4 of 7) and RB1 (5 of 7) were seen. By contrast, none 
of these mutations were found in 11 well-differentiated PanNETs. Abnormal expression of the TP53 (95\%) and RB1 (75\%) proteins was also frequently seen in PanNEC, but not in welldifferentiated PanNETs. Furthermore, all PanNECs retained expression of ATRX and DAXX, while, as noted above, PanNETs showed loss of expression of ATRX and DAXX in $45 \%$ of cases (Table 4) (78).

As mentioned earlier, sporadic PanNETs likely develop through a similar hyperplasianeoplasia sequence as familial PanNETs. MEN1 syndrome associated PanNETs show loss of the wild-type MEN1 allele in up to $100 \%$ of cases (compared to $19-44 \%$ in sporadic PanNETs). Loss of the wild-type MEN1 allele is also seen in microadenomas of MEN1 patients and is therefore an early event $(83,92-94)$. Loss of Menin can be demonstrated by immunohistochemistry (Figure $4 C$ and D). In non-syndromic patients, it is unclear which initiating events cause microadenomas to develop, also bearing in mind that not all sporadic PanNETs have MEN1 alterations.

ATRX and DAXX mutations with ALT activation have been reported to correlate significantly with tumor size and T-stage, and are thus considered a late event in tumor progression. In total $45 \%$ of PanNETs have alterations in one of both genes $(86,87)$. Although less likely, it is not known if sporadic microadenomas have ATRX or DAXX alterations.

In contrast to sporadic PanNETs, ATRX and DAXX alterations were only seen in $6 \%$ of PanNETs from MEN1 syndrome patients (but also as late event) and in $0 \%$ of microadenomas suggesting a less important role for these alterations in MEN1 syndrome tumor progression (95).

\section{Epigenetic alterations}

Few studies investigated epigenetic alterations in PanNETs. Hypomethylation in LINE-1 was reported in $20 \%$ of well-differentiated PanNETs, and strongly correlated with poor prognosis and high stage (96). Other studies found hypermethylation of the tumor suppressor gene RASSF1A in 75-80\% of PanNETs with associated decreased protein expression of RASSF1A $(97,98)$. Interestingly, the RASSF1 gene has six other transcriptional variants (B$G)$, of which RASSF1C was seen to be expressed 10 times higher in PanNET than in normal tissue (98). An in vitro study found the balance between isoform $A$ and $C$ crucial for the expression of $\beta$-catenin, where silencing RASSF1A and expression of RASSF1C promotes the accumulation of $\beta$-catenin by inhibiting its $\mathrm{hTrCP}$ mediated proteasomal degradation (99), possibly sustaining Wnt signaling in PanNET. RASSF1A furthermore represses miR- 
21 (100). Interestingly overexpression of miR-21 which is also upregulated in PDAC, was strongly associated with both a high Ki67 proliferation index and metastasis to the liver (101), potentially giving the RAS pathway a role in higher grades of PanNET (54).

\section{MicroRNA}

Studies of microRNA expression have suggested that miR-193b is a differential marker for PanNET in tissue and serum compared to normal (102). MiR-103 and miR-107 were also overexpressed and miR-155 was downregulated in PanNET (101).

\section{Changes in gene expression}

Analyses of gene-expression patterns in PanNETs have found that a number of genes are upregulated in PanNETs, including oncogenes (e.g. MLLT10/AF10), cell adhesion molecules (e.g. fibronectin) and growth factors (e.g. IGFBP3) compared to normal islets. Growth factor IGFBP3 was upregulated significantly more in metastases compared to primary PanNETs. In addition, downregulation of tumor suppressor genes (NME3), cell checkpoint proteins (p21/Cip1), and transcription factor JunD that is inhibited by Menin, have been reported (103). Comparison of gene expression between sporadic PanNETs and VHL associated PanNETs, found that VHL associated tumors follow a specific pathway with upregulation of genes related to hypoxia inducible factor proteins (HIF) and vascular endothelial growth factor (VEGF), both of which regulate angiogenesis (104).

Therapeutically, PanNETs relying on angiogenesis are theoretically targetable by blocking specific pathway components (e.g. VEGF inhibitors) (105-107). Similarly, PanNETs relying on mTOR activation should be particularly susceptible to everolimus, a drug which has shown to significantly prolong survival (108).

\section{SOLID-PSEUDOPAPILLARY NEOPLASMS}

Solid-pseudopapillary neoplasms (SPN) are rare tumors accounting for 1-2\% of all malignant neoplasms of the pancreas. These neoplasms mostly occur in female (90\%) patients at an average age of 29 years (SD: 14). SPNs have a low malignant potential. SPNs are usually limited to the pancreas, but $8 \%$ of patients present with distant metastasis. Disease free survival after curative resection is $95 \%$ (109).

\section{Gross and microscopic findings}

SPNs are essentially solid neoplasms that often undergo dramatic cystic degeneration 

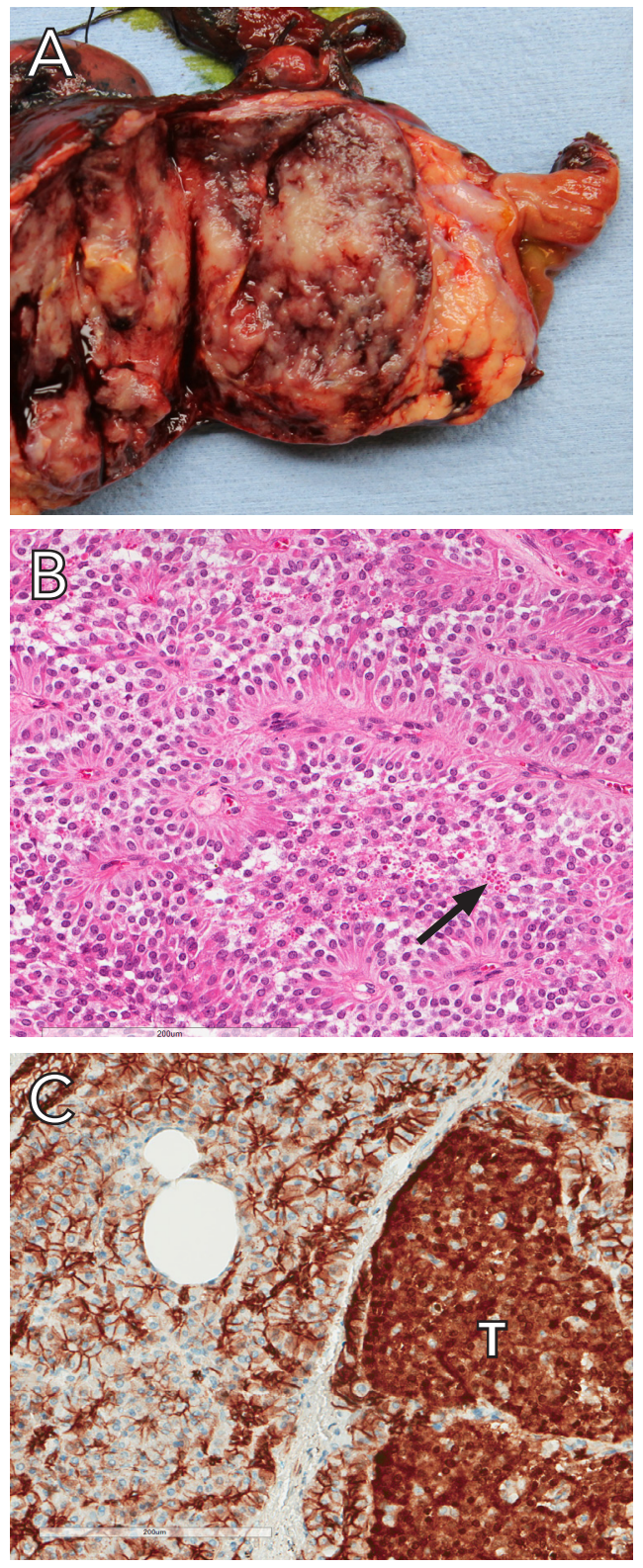

Figure 5. A, macroscopic appearance of a solid-pseudopapillary neoplasm showing a well demarcated tumor with solid, pseudopapillary and hemorrhagic-necrotic pseudocystic structures. B, microscopically, SPN is characterized by solid areas with relatively uniform cells with eosinophilic or clear vacuolated cytoplasm admixed with delicate capillaries and areas with extensive degenerative changes. The cells are poorly cohesive causing the pseudopapillary appearance. Note the eosinophilic globules (arrow). C, nuclear $\beta$-catenin expression in SPN (T, tumor) and normal membranous labelling in adjacent normal pancreatic parenchyma. 
creating a gross lesion with a mixture of solid, pseudopapillary and hemorrhagic-necrotic areas (Figure 5A). Microscopically, these neoplasms are composed of poorly cohesive uniform cells clinging ineffectively to delicate capillaries surrounded by extensive degenerative changes. The cells have eosinophilic or clear vacuolated cytoplasm, and the nuclei are round to oval and can be often grooved or indented. Rarely the nuclei are bizarre appearing in areas with degeneration. Eosinophilic globules and foamy macrophages are typically present in these neoplasms (Figure 5B). SPNs can be distinguished from other pancreatic tumors by the expression of CD10, paranuclear dot-like CD99 labeling and abnormal nuclear labeling for $\beta$-catenin (Figure $5 \mathrm{C}$ ) or lymphoid enhancer-binding factor 1 (LEF1) (110-114). The microscopic differential diagnosis consists of neoplasms with a solid and cellular appearance like pancreatic neuroendocrine tumor, acinar cell carcinoma and pancreatoblastoma (Table 1).

\section{Genetic Signatures: Sporadic and Familial SPN}

Activating mutations in CTNNB1 ( $\beta$-catenin) occur in virtually all SPNs, reflected by the nuclear accumulation of $\beta$-catenin seen in immunohistochemistry $(115,116)$. Recent whole exome sequencing of SPNs found on average of only three non-synonymous mutations per tumor, which is extremely low compared to all of the other pancreatic neoplasms. The CTNNB1 gene mutations all occur in the critical region between codons 32 and 37 preventing phosphorylation and subsequent degradation of the $\beta$-catenin protein.

Two SPNs have been reported in patients with Familial Adenomatous Polyposis (FAP), caused by germline APC mutations, confirming that an APC mutation is also capable of driving SPN development $(117,118)$. The female predominance of SPN is not understood, but it has been shown that estrogenic molecules can influence proliferation in vitro (119).

\section{Epigenetic Alterations}

Undegraded $\beta$-catenin in SPNs forms a complex with LEF1, enters the nucleus and activates transcription of several oncogenes including cyclin-D1 that is overexpressed in $70-100 \%$ of SPNs $(115,116,120)$. Cyclin-D1 and its cyclin-dependent kinases phosphorylate the Retinoblastoma $(\mathrm{Rb})$ protein, which drives the cell in the S-phase of the cell cycle. P21 and P27, known to inhibit Rb phosphorylation, were shown to be upregulated in $86 \%$ and $100 \%$ of SPNs, respectively. Interestingly, hyperphosphorylated $\mathrm{Rb}$ was not detectable, which might explain the low growth-rate of SPN compared to other $\beta$-catenin mutated tumors (121). 

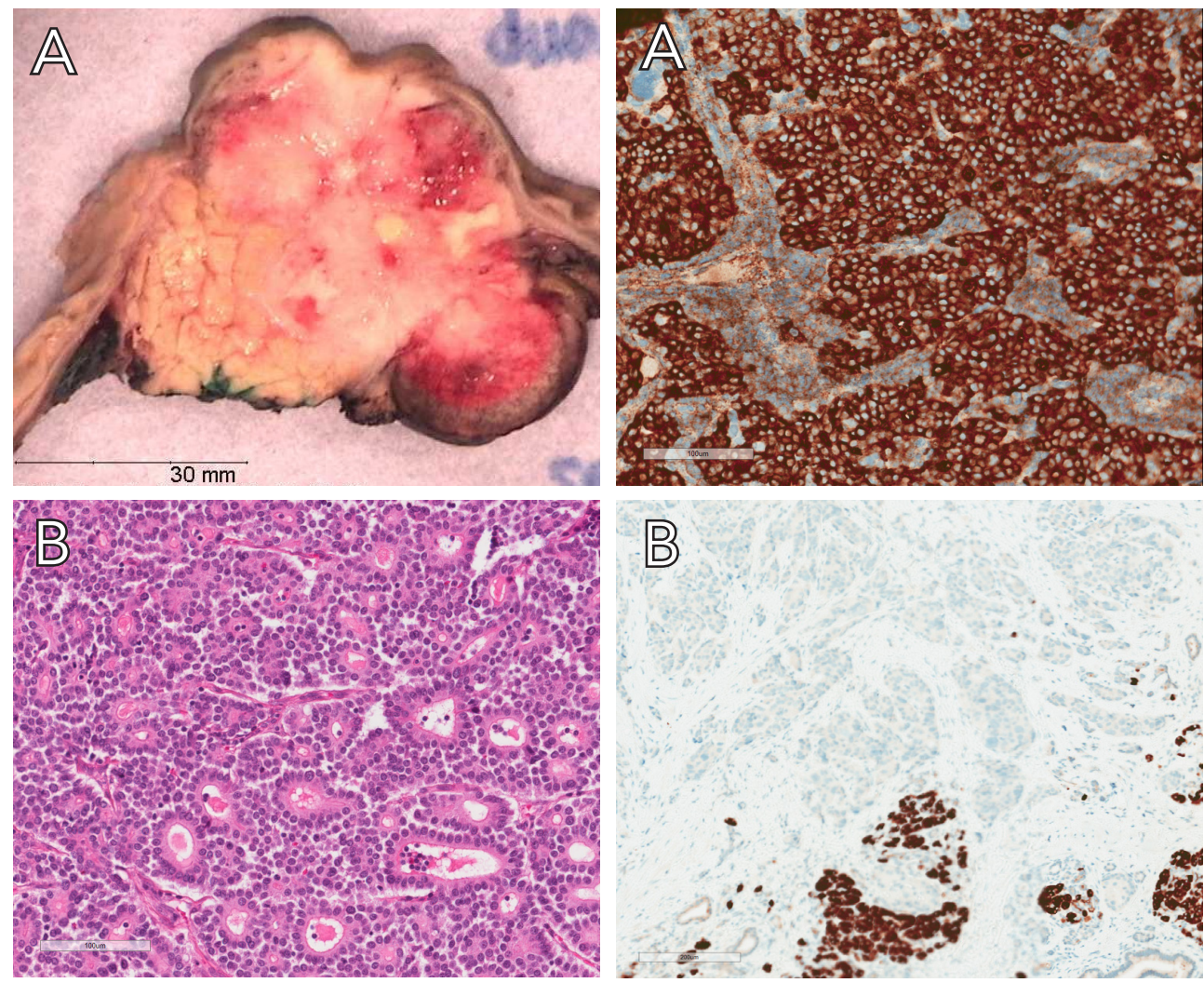

Figure 6. A, macroscopic appearance of an acinar

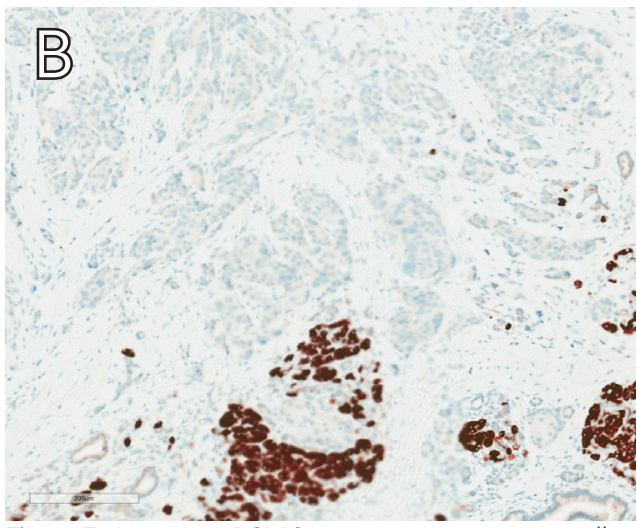

Figure 7. $A$, positive $B C L 10$ expression in an acinar cell cell carcinoma. B, microphotograph of an acinar cell carcinoma characterized by a cells with granular cytoplasm and round to oval uniform nuclei forming carcinoma. B, negative BCL10 expression in a pancreatic neuroendocrine tumor.

form small acinar structures.

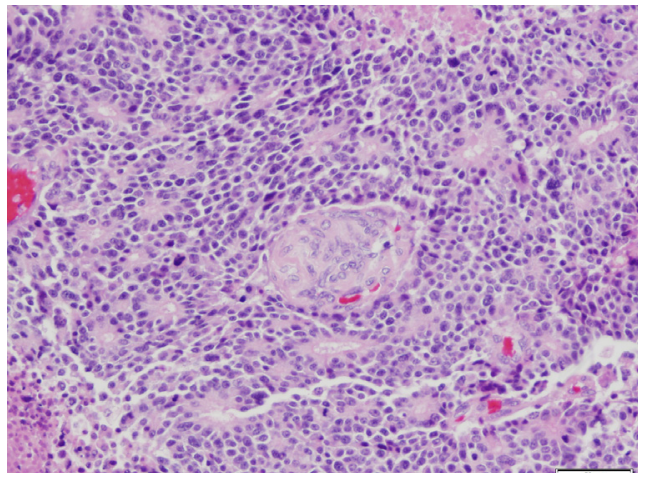

Figure 8. Microphotograph of pancreatoblastoma showing characteristic squamoid nests. 


\section{MicroRNA and changes in gene expression}

Few studies investigated gene expression and epigenetic alterations in SPN, and all are complicated by the fact that the normal cell that is the counterpart of the neoplastic cell in SPNs has not been identified. These studies are therefore, at best, comparing apples to oranges. One study investigated mRNA and miR expression in 14 SPNs and found 1686 genes to be differentially expressed compared to normal pancreatic parenchyma (which is composed mostly of acinar cells) (122). These differentially expressed genes activated the Wnt pathway, Hedgehog $(\mathrm{HH})$ pathway, androgen-receptor $(\mathrm{AR})$ pathway and epithelial mesenchymal transition. Moreover, 79 miRs were differentially expressed in these SPNs (49 miRs upregulated, 30 miRs downregulated). By predicting miR targets, 17 of the 30 downregulated miRs possibly upregulated mRNAs in the Wnt/HH/AR pathways (123). A proteomic profile did not significantly confirm these pathways, but did find upregulation of several proteins involved in the Wnt pathway (122). Another mRNA analysis in SPN found the NOTCH pathway to be activated in addition to the Wnt Pathway (124). Large chromosomal rearrangements, aberrant methylation or other non-coding RNAs have not been investigated in SPN.

\section{ACINAR CELL CARCINOMA}

Acinar cell carcinoma (ACC) is a rare neoplasm accounting for $<1 \%$ of malignant pancreatic tumors. Median age of presentation is 56 years (SD: 15). Most cases occur in late adulthood, but $6 \%$ of cases occur between 8 and 15 years of age. There is no clear syndrome associated with ACC, but ACC has been reported in patients with Peutz-Jeghers syndrome, Lynch syndrome, Familial Adenomatous Polyposis, and in a patient with a germline BRCA1 mutation (125-128).

Although $60 \%$ of patients with ACC have distant metastasis at presentation (similar to PDAC), overall 5-year survival is 45\% (129). Some ACCs release digestive enzymes and other products into the blood stream, including alpha-fetoprotein and lipase $(130,131)$. About $15 \%$ of patients with ACC present with metastatic fat necrosis, peripheral eosinophilia and arthralgias caused by elevated serum lipase (132).

\section{Gross and microscopic findings}

Compared to PDAC, ACCs are relatively soft and well-circumscribed tumors. Microscopically, ACCs are reminiscent of normal exocrine pancreatic cells with enlarged uniform nuclei with prominent nucleoli and finely granular eosinophilic cytoplasm. The cells can form small 
acinar units or sheets without a distinctive architecture (Figure 6A and B) (5). Acinar cell carcinomas express pancreatic exocrine enzymes such as trypsin, chymotrypsin and lipase that can be detectable by immunohistochemistry (132). BCL10, normally expressed in normal acini, is also expressed in ACC and is helpful in the differential diagnosis between ACC and other pancreatic neoplasms such as PanNET and PDAC (Figure 7A and B) (133, 134). Also the monoclonal antibody 2P-1-2-1 can be used to show acinar differentiation (135). The microscopic differential diagnosis consists of neoplasms with a solid and cellular appearance like pancreatic neuroendocrine tumor, solid pseudopapillary neoplasm, pancreatoblastoma (Table 1).

\section{Genetic Signature: Sporadic ACC}

Whole exome sequencing of ACCs revealed that these tumors, on average, harbor a large number of mutations (131 nonsynonymous somatic mutations per tumor in one study). Also, chromosomal instability is seen with a relative high fractional allelic loss compared to PDAC. Chromosome $11 \mathrm{p}$ is lost in $~ 50 \%$ of ACC, suggesting that a locus on $11 \mathrm{p}$ may play an important role in ACC development $(136,137)$. Many other gains and losses have been reported including loss of the TP53 locus on 17p (25\%), the APC locus on $5 q 21$ (50\%), the SMAD4 locus on $18 q(60 \%)$ and gain of the CTNNB1 ( $\beta$-catenin) locus on 3p (137-140). Whole exome sequencing data further revealed that no single gene was mutated in more than $30 \%$ of ACCs. The genes targeted include SMAD4 (25\%); JAK1 (20\%); BRAF, RB1, TP53 (13\% each); APC, ARID1A, GNAS, MLL3, PTEN (9\% each) and ATM, BAP1, BRCA2 PALB2, MEN1, RNF43 (4\% each) (137). Recently, a review combined all ACC sequencing studies and found similar results: SMAD4 mutations in 19\% of ACC, CTNNB1/APC in 15\%, TP53 in $12 \%$, and BRAF in 6\% (139).

Ten percent of ACCs appear to be microsatellite instable and may thus be sensitive to immunotherapy $(19,139)$. In addition, a number of other potentially actionable mutations, such as BRCA and JAK1 mutations, have been found in ACCs (137). BRAF mutations are rarely seen in $A C C$; notably, comprehensive genomic profiling identified rearrangements in $23 \%$ of ACC involving either BRAF or RAS. The most prevalent fusion SND1-BRAF activated the MAPK pathway and made the cells sensitive for MEK inhibitor trametinib, so this pathway might be useful as therapeutic target for a subgroup of patients with ACC (141).

\section{Epigenetic alterations and MicroRNA}

The importance of the APC/ $\beta$-catenin pathway for ACC becomes more evident when 
methylation is taken into account. RASSF1 and APC were reported to be methylated in $60 \%$ and $56 \%$ of ACCs, respectively (142). A different study confirmed the high percentage of ACC with APC methylation, and also found significantly more MLH1 methylation in ACC compared to PDAC and PanNET (143).

MiR has only been studied in four ACCs in comparison to PanNETs. Surprisingly, 93\% of differentially upregulated miRs and $70 \%$ of differentially downregulated miRs in ACC compared to normal pancreas were also up- or downregulated in PanNET. No specific miR was up- or downregulated in ACC versus PanNET. Overexpression of miR-17, miR-20, miR21, miR-92-1, miR-103 and miR-107; and lack of expression of miR-155 was found in ACC (101).

\section{PANCREATOBLASTOMA}

Pancreatoblastoma (PB) is another rare tumor with acinar differentiation. PBs usually present in childhood at an average age of 5 years (SD: 2), but there is also a rare group that presents at adult age $(144,145)$. The overall 5 year survival is approximately $50 \%$. PB is associated with Beckwith-Wiedemann syndrome, an (epi)genetic overgrowth-cancer predisposition disorder characterized by exomphalos, macroglossia, and gigantism (146). As in ACC, serum alpha-fetoprotein and lipase can be elevated in PB and pancreatic panniculitis has also been reported.

\section{Gross and microscopic findings}

Tumors are very similar to ACC in their acinar differentiation. The distinguishing element in PB from other tumors with acinar differentiation are characteristic squamoid nests, which can vary in size and appearance and can even show keratinization (Figure 8). Neuroendocrine or ductal components may also be encountered, but acinar differentiation and squamoid nests are both required for the diagnosis. PB shares the same immunohistochemical markers for acinar differentiation with $A C C$, but can also stain positive for markers of ductal or neuroendocrine differentiation. SMAD4 expression is immunohistochemically lost in $20 \%$ (147), and abnormal nuclear expression of $\beta$-catenin can be seen, sometimes in the squamoid nests $(5,127)$. The microscopic differential diagnosis consists of neoplasms with a solid and cellular appearance like pancreatic neuroendocrine tumor, solid pseudopapillary neoplasm and acinar cell carcinoma (Table 1). In children, other tumors like Wilms tumor and hepatoblastoma should be considered. 


\section{Genetic signature and epigenetic alterations}

Patients with Beckwith-Wiedemann syndrome (germline loss of heterozygosity of chromosome 11p) have a significantly higher risk of pediatric tumors, amongst others pancreatoblastoma which has been reported in several BWS patients $(144,146)$. Interestingly loss of $11 \mathrm{p}$ also occurs in more than $80 \%$ of sporadic PBs (147). Likely, several genes on $11 p$ that are expressed according to their parental origin (imprinting) play a role in PB tumorigenesis (148). The APC/ $\beta$-catenin pathway also plays an important role with $40 \%$ to $60 \%$ of sporadic PBs having mutations in CTNN1B. In addition, a case with biallelic inactivation of $A P C$ in a FAP patient has been reported $(115,147)$. Aberrant methylation of the promoter RASSF1A was seen in in 2 cases $(149,150)$. No further characterization in epigenetics has been done.

\section{CONCLUSIONS}

The underlying alterations of pancreatic cancer demonstrate that the traditional histopathologic classification of these neoplasms has a solid genetic basis. The genetic changes within each tumor type add to the pathologic classification with the identification of new prognostic markers and new therapeutic targets.

Even with all of the advances in our understanding of genetics of pancreatic neoplasms, the cornerstone to a correct diagnosis is still traditional gross and microscopic examination. Especially the importance of gross inspection is often less appreciated, and yet this can already give important clues to a correct diagnosis. For instance, some tumors are typically solid whereas others are typically cystic. Also, location of a tumor in the pancreas (head, body of tail) and whether a tumor is well circumscribed or ill-defined can point in a certain direction. Most diagnoses of pancreatic tumors can be made without help of additional genetic studies although sometimes proof of a specific genetic alteration in a tumor can further establish a presumed diagnosis. The best example clearly is the SPN, in which virtually all cases have the same underlying CTNNB1 mutation and immunohistochemistry for $\beta$-catenin is routinely used in the diagnostic workup. Also, loss of SMAD4 immunohistochemistry is frequently used in daily practice to suggest pancreatic origin of an adenocarcinoma in a distant site in a patient with a pancreatic mass.

Slowly we are heading towards an era where the combination of classical morphologic pathology and genetic characterization will be essential to establish a more accurate diagnosis. Furthermore, genetic profiling is becoming more and more important for 
treatment choices; for instance with the choice for a targeted therapy, such as mTOR inhibitors in pancreatic neuroendocrine tumors or PARP inhibitors in BRCA deficient tumors. In the near future, stromal activation, miRNA and methylation markers might influence our choices by better predicting tumor behavior and prognosis. Ideally, would use our knowledge of genetic and epigenetic alterations to screen the blood and pancreatic juices for genetic alteration that identify patients with a high-risk precursor lesion or an early form of cancer. Although our understanding of the genetics of pancreatic cancer has immensely increased in the last decade, many years of research are still needed to integrate all this knowledge and translate it into day-to-day practice.

\section{COMPETING INTERESTS}

WMH, LAAB and GJAO have no competing interests to declare. $\mathrm{RHH}$ receives royalty payments from Myriad Genetics for the PALB2 invention, and RHH is on the Board of MiDiagnostics. Both relationships are overseen by the Johns Hopkins Office of Policy Coordination.

\section{ACKNOWLEDGEMENTS}

Dutch Cancer Society (KWF), Lisa Waller Hayes Foundation, Nijbakker-Morra foundation, Dutch Digestive Foundation (MLDS; CDG 14-02)

\section{REFERENCES}

1. Yu J, Blackford AL, Dal Molin M, Wolfgang CL, Goggins M. Time to progression of pancreatic ductal adenocarcinoma from low-to-high tumour stages. Gut. 2015;64(11):1783-9.

2. Siegel RL, Miller KD, Jemal A. Cancer statistics, 2015 CA Cancer J Clin. 2015;65(1):5-29.

3. Vincent A, Herman J, Schulick R, Hruban RH, Goggins M. Pancreatic cancer. Lancet. 2011;378(9791):607-20.

4. Rahib L, Smith BD, Aizenberg R, Rosenzweig AB,

Fleshman JM, Matrisian LM. Projecting cancer incidence and deaths to 2030: the unexpected burden of thyroid, liver, and pancreas cancers in the United States. Cancer Res. 2014;74(11):291321.

5. Bosman FT, Carneiro F, Hruban RH. WHO Classification of Tumours of the Digestive System: World Health Organization; $2010.417 \mathrm{p}$.

6. Hruban RH, Pitman MB, Klimstra DS. Tumors of the Pancreas: American Registry of Pathology; 2007 Jan 01.422 p. 7. Terada T, Ohta T, Sasaki M, Nakanuma Y, Kim YS. Expression of MUC apomucins in normal pancreas and pancreatic tumours. J Pathol. 1996;180(2):160-5.

8. Nagata K, Horinouchi M, Saitou M, Higashi M, Nomoto M, Goto M, et al. Mucin expression profile in pancreatic cancer and the precursor lesions. J Hepatobiliary Pancreat Surg 2007;14(3):243-54.

9. Takeda S, Nakao A, Ichihara T, Suzuki Y, Nonami T, Harada A, et al. Serum concentration and immunohistochemical localization of SPan-1 antigen in pancreatic cancer. A comparison with CA19-9 antigen. Hepatogastroenterology. 1991;38(2):143-8. 10. Brosens LA, Hackeng WM, Offerhaus GJ, Hruban RH, Wood LD. Pancreatic adenocarcinoma pathology: changing "landscape". J Gastrointest Oncol. 2015;6(4):358-74.

11. Basturk O, Hong SM, Wood LD, Adsay NV, Albores-Saavedra J, Biankin AV, et al. A Revised Classification System and Recommendations From the Baltimore Consensus Meeting for Neoplastic Precursor Lesions in the Pancreas. Am J Surg Pathol. 2015;39(12):1730-41.

12. Roberts NJ, Norris AL, Petersen GM, Bondy ML, Brand R, Gallinger S, et al. Whole Genome Sequencing Defines the Genetic Heterogeneity of Familial Pancreatic Cancer. Cancer Discov. 2016;6(2):166-75.

13. Jones $\mathrm{S}$, Hruban RH, Kamiyama M, Borges M, Zhang XS, Parsons DW, et al. Exomic Sequencing Identifies PALB2 as a Pancreatic Cancer Susceptibility Gene. Science. 2009;324(5924):217-. 
14. Zhen DB, Rabe KG, Gallinger S, Syngal S, Schwartz AG, Goggins MG, et al. BRCA1, BRCA2, PALB2, and CDKN2A mutations in familial pancreatic cancer: a PACGENE study. Genet Med. 2015;17(7):569-77.

15. Roberts NJ, Jiao Y, Yu J, Kopelovich L, Petersen GM, Bondy ML, et al. ATM mutations in patients with hereditary pancre atic cancer. Cancer Discov. 2012;2(1):41-6.

16. Hearle N, Schumacher V, Menko FH, Olschwang S, Boardman LA, Gille JJ, et al. Frequency and spectrum of cancers in the Peutz-Jeghers syndrome. Clin Cancer Res. 2006;12(10):320915.

17. Kastrinos F, Mukherjee B, Tayob N, Wang F, Sparr J,

Raymond VM, et al. Risk of pancreatic cancer in families with Lynch syndrome. JAMA. 2009;302(16):1790-5.

18. Canto MI, Harinck F, Hruban RH, Offerhaus GJ, Poley JW, Kamel I, et al. International Cancer of the Pancreas Screening (CAPS) Consortium summit on the management of patients with increased risk for familial pancreatic cancer. Gut. 2013;62(3):33947.

19. Le DT, Uram JN, Wang H, Bartlett BR, Kemberling $H$, Eyring AD, et al. PD-1 Blockade in Tumors with Mismatch-Repair Deficiency. N Engl J Med. 2015;372(26):2509-20.

20. Bhalla A, Saif MW. PARP-inhibitors in BRCA-associated pancreatic cancer. JOP. 2014;15(4):340-3.

21. Waddell N, Pajic M, Patch AM, Chang DK, Kassahn $\mathrm{KS}$, Bailey $\mathrm{P}$, et al. Whole genomes redefine the mutational landscape of pancreatic cancer. Nature. 2015;518(7540):495-501.

22. Childs EJ, Mocci E, Campa D, Bracci PM, Gallinger S, Goggins M, et al. Common variation at 2p13.3, 3q29, 7p13 and $17 q 25.1$ associated with susceptibility to pancreatic cancer. Nature Genetics. 2015;47(8):911-+.

23. Wolpin BM, Rizzato C, Kraft P, Kooperberg C, Petersen GM, Wang Z, et al. Genome-wide association study identifies multiple susceptibility loci for pancreatic cancer. Nat Genet. 2014;46(9):994-1000.

24. Wolpin BM, Kraft P, Xu M, Steplowski E, Olsson $M L$, Arslan AA, et al. Variant $A B O$ blood group alleles, secretor status, and risk of pancreatic cancer: results from the pancreatic cancer cohort consortium. Cancer Epidemiol Biomarkers Prev. 2010;19(12):3140-9

25. Biankin AV, Waddell N, Kassahn KS, Gingras MC, Muthuswamy LB, Johns AL, et al. Pancreatic cancer genomes reveal aberrations in axon guidance pathway genes. Nature. 2012;491(7424):399-405.

26. Jones S, Zhang X, Parsons DW, Lin JC, Leary RJ,

Angenendt $P$, et al. Core signaling pathways in human pancreatic cancers revealed by global genomic analyses. Science. 2008;321(5897):1801-6.

27. Witkiewicz AK, McMillan EA, Balaji U, Baek G, Lin WC, Mansour J, et al. Whole-exome sequencing of pancreatic cancer defines genetic diversity and therapeutic targets. Nat Commun. 2015;6:6744.

28. Griffin CA, Hruban RH, Morsberger LA, Ellingham T, Long PP, Jaffee EM, et al. Consistent chromosome abnormalities in adenocarcinoma of the pancreas. Cancer Res. 1995;55(11):23949.

29. Sausen M, Phallen J, Adleff V, Jones S, Leary RJ,

Barrett MT, et al. Clinical implications of genomic alterations in the tumour and circulation of pancreatic cancer patients. Nat Commun. 2015;6:7686.

30. Blackford A, Serrano OK, Wolfgang CL, Parmigiani G, Jones S, Zhang $X$, et al. SMAD4 gene mutations are associated with poor prognosis in pancreatic cancer. Clin Cancer Res. 2009; 15(14):4674-9.

31. Castro-Giner F, Ratcliffe P, Tomlinson I. The mini-driver model of polygenic cancer evolution. Nat Rev Cancer. 2015;15(11):680-5.

32. Yachida S, Jones S, Bozic I, Antal T, Leary R, Fu B, et al. Distant metastasis occurs late during the genetic evolution of pancreatic cancer. Nature. 2010;467(7319):1114-7.

33. Bettegowda C, Sausen M, Leary RJ, Kinde I,

Wang Y, Agrawal N, et al. Detection of circulating tumor DNA in early- and late-stage human malignancies. Sci Transl Med. 2014;6(224):224ra24.

34. Springer S, Wang Y, Dal Molin M, Masica DL, Jiao $Y$, Kinde I, et al. A combination of molecular markers and clinical features improve the classification of pancreatic cysts. Gastroenterology. 2015;149(6):1501-10.

35. Shimizu H, Horii A, Sunamura M, Motoi F, Egawa S, Unno M, et al. Identification of epigenetically silenced genes in human pancreatic cancer by a novel method "microarray coupled with methyl-CpG targeted transcriptional activation" (MeTA-array). Biochem Biophys Res Commun. 2011;411(1):162-7.

36. Tan AC, Jimeno A, Lin SH, Wheelhouse J, Chan F, Solomon A, et al. Characterizing DNA methylation patterns in pancreatic cancer genome. Mol Oncol. 2009;3(5-6):425-38.

37. Vincent A, Omura N, Hong SM, Jaffe A, Eshleman J,

Goggins M. Genome-wide analysis of promoter methylation associated with gene expression profile in pancreatic adenocarcinoma. Clin Cancer Res. 2011;17(13):4341-54.

38. Nones K, Waddell N, Song S, Patch AM, Miller D, Johns A, et al. Genome-wide DNA methylation patterns in pancreatic ductal adenocarcinoma reveal epigenetic deregulation of SLIT-ROBO, ITGA2 and MET signaling. Int J Cancer. 2014;135(5):1110-8.

39. Omura N, Li CP, Li A, Hong SM, Walter K, Jimeno A, et al. Genome-wide profiling of methylated promoters in pancreatic adenocarcinoma. Cancer Biol Ther. 2008;7(7):1146-56.

40. Zhao YX, Sun JF, Zhang HY, Guo SC, Gu J, Wang W, et al. High-frequency aberrantly methylated targets in pancreatic adenocarcinoma identified via global DNA methylation analysis using methylCap-seq. Clin Epigenetics. 2014;6(1):18.

41. Sato N, Fukushima N, Maitra A, Matsubayashi H,

Yeo CJ, Cameron JL, et al. Discovery of novel targets for aberrant methylation in pancreatic carcinoma using high-throughput microarrays. Cancer Research. 2003;63(13):3735-42.

42. Goonesekere NCW, Wang XS, Ludwig L, Guda

C. A Meta Analysis of Pancreatic Microarray Datasets Yields New Targets as Cancer Genes and Biomarkers. Plos One. 2014;9(4):e93046.

43. Hong SM, Park JY, Hruban RH, Goggins M. Molecular signatures of pancreatic cancer. Arch Pathol Lab Med. $2011 ; 135(6): 716-27$

44. Fukushima N, Sato N, Ueki T, Rosty C, Walter KM,

Wilentz RE, et al. Aberrant methylation of preproenkephalin and p16 genes in pancreatic intraepithelial neoplasia and pancreatic 
ductal adenocarcinoma. Am J Pathol. 2002;160(5):1573-81.

45. Moore PS, Sipos B, Orlandini S, Sorio C, Real FX,

Lemoine NR, et al. Genetic profile of 22 pancreatic carcinoma cell lines. Analysis of K-ras, p53, p16 and DPC4/Smad4. Virchows Arch. 2001;439(6):798-802.

46. Ueki T, Toyota M, Sohn T, Yeo CJ, Issa JP, Hruban RH, et al. Hypermethylation of multiple genes in pancreatic adenocarcinoma. Cancer Res. 2000;60(7):1835-9.

47. Matsubayashi H, Canto M, Sato N, Klein A, Abe T, Yamashita K, et al. DNA methylation alterations in the pancreatic juice of patients with suspected pancreatic disease. Cancer Res. 2006;66(2):1208-17.

48. Habbe N, Koorstra JBM, Mendell JT, Offerhaus GJ, Ryu JK, Feldmann G, et al. MicroRNA miR-155 is a biomarker of early pancreatic neoplasia. Cancer Biol Ther. 2009;8(4):340-6.

49. Frampton AE, Krell J, Jamieson NB, Gall TM, Giovannetti $E$, Funel $N$, et al. microRNAs with prognostic significance in pancreatic ductal adenocarcinoma: A meta-analysis. Eur J Cancer. 2015;51(11):1389-404.

50. Ma MZ, Kong X, Weng MZ, Cheng K, Gong W, Quan ZW, et al. Candidate microRNA biomarkers of pancreatic ductal adenocarcinoma: meta-analysis, experimental validation and clinical significance. J Exp Clin Cancer Res. 2013;32(1):71. 51. Yang JY, Sun YW, Liu DJ, Zhang JF, Li J, Hua R. MicroRNAs in stool samples as potential screening biomarkers for pancreatic ductal adenocarcinoma cancer. Am J Cancer Res. 2014;4(6):663-73.

52. Abue M, Yokoyama M, Shibuya R, Tamai K, Yamaguchi K, Sato I, et al. Circulating miR-483-3p and miR-21 is highly expressed in plasma of pancreatic cancer. Int J Oncol. 2014.

53. Volinia S, Calin GA, Liu CG, Ambs S, Cimmino A Petrocca F, et al. A microRNA expression signature of human solid tumors defines cancer gene targets. Proc Natl Acad Sci U S A. 2006; 103(7):2257-61.

54. Hatley ME, Patrick DM, Garcia MR, Richardson JA, Bassel-Duby R, van Rooij E, et al. Modulation of K-Ras-dependent lung tumorigenesis by MicroRNA-21. Cancer Cell. 2010;18(3):28293.

55. Selcuklu SD, Donoghue MTA, Spillane C. miR-21 as a key regulator of oncogenic processes. Biochem Soc T. 2009;37(Pt 4):918-25.

56. Toste PA, Li L, Kadera BE, Nguyen AH, Tran LM, Wu $\mathrm{N}$, et al. p85a is a microRNA target and affects chemosensitivity in pancreatic cancer. J Surg Res. 2015;196(2):285-93.

57. Wang CP, Sun Y, Wu HW, Yu SN, Zhang L, Meng YX, et al. Elevated miR-483-3p expression is an early event and indicates poor prognosis in pancreatic ductal adenocarcinoma. Tumor Biol. 2015;36(12):9447-56.

58. Bai ZH, Sun JL, Wang XB, Wang H, Pei HH, Zhang ZL. MicroRNA-153 is a prognostic marker and inhibits cell migration and invasion by targeting SNAI1 in human pancreatic ductal adenocarcinoma. Oncol Rep. 2015;34(2):595-602.

59. Xia X, Zhang K, Cen G, Jiang T, Cao J, Huang K, et al. MicroRNA-301a-3p promotes pancreatic cancer progression via negative regulation of SMAD4. Oncotarget. 2015;6(25):21046-63. 60. Zhu Z, Xu Y, Zhao J, Liu Q, Feng W, Fan J, et al. miR367 promotes epithelial-to-mesenchymal transition and invasion of pancreatic ductal adenocarcinoma cells by targeting the
Smad7-TGF- $\beta$ signalling pathway. Br J Cancer. 2015;112(8):136775.

61. Müller S, Raulefs S, Bruns P, Afonso-Grunz F, Plötner $A$, Thermann $R$, et al. Next-generation sequencing reveals novel differentially regulated mRNAs, IncRNAs, miRNAs, sdRNAs and a piRNA in pancreatic cancer. Mol Cancer. 2015;14(1):94.

62. Collisson EA, Sadanandam A, Olson P, Gibb WJ,

Truitt M, Gu SD, et al. Subtypes of pancreatic ductal adenocarcinoma and their differing responses to therapy. Nature Medicine. 2011;17(4):500-U140.

63. Moffitt RA, Marayati R, Flate EL, Volmar KE, Loeza SG, Hoadley KA, et al. Virtual microdissection identifies distinct tumorand stroma-specific subtypes of pancreatic ductal adenocarcinoma. Nat Genet. 2015;47(10):1168-78.

64. Harsha HC, Kandasamy K, Ranganathan P, Rani S,

Ramabadran S, Gollapudi S, et al. A compendium of potential biomarkers of pancreatic cancer. PLoS Med. 2009;6(4):e1000046. 65. Wang $Q$, Chaerkady R, Wu JA, Hwang HJ,

Papadopoulos N, Kopelovich L, et al. Mutant proteins as cancer-specific biomarkers. P Natl Acad Sci USA. 2011;108(6):2444-9. 66. Zhang Y, Choi M. Immune Therapy in Pancreatic Cancer: Now and the Future? Rev Recent Clin Trials. 2015;10(4):31725 .

67. Rosenberg SA, Restifo NP. Adoptive cell transfer as personalized immunotherapy for human cancer. Science. 2015;348(6230):62-8.

68. Xu Z, Pothula SP, Wilson JS, Apte MV. Pancreatic cancer and its stroma: a conspiracy theory. World J Gastroenterol. 2014;20(32):11216-29.

69. Rhim AD, Oberstein PE, Thomas DH, Mirek ET, Palermo CF, Sastra SA, et al. Stromal Elements Act to Restrain, Rather Than Support, Pancreatic Ductal Adenocarcinoma. Cancer Cell. 2014;25(6):735-47.

70. Stromnes IM, DelGiorno KE, Greenberg PD, Hingorani SR. Stromal reengineering to treat pancreas cancer. Carcinogenesis. 2014;35(7):1451-60.

71. Halfdanarson TR, Rabe KG, Rubin J, Petersen GM.

Pancreatic neuroendocrine tumors (PNETs): incidence, prognosis and recent trend toward improved survival. Ann Oncol. 2008;19(10):1727-33.

72. Ries L, Young JJ, Keel G, Eisner M, Lin Y, Horner M Cancer Survival Among Adults: US Department of Health and Human Services, National Institutes of Health, National Cancer Institute.; 2007.

73. Falconi M, Eriksson B, Kaltsas G, Bartsch DK, Capdevila J, Caplin M, et al. ENETS Consensus Guidelines Update for the Management of Patients with Functional Pancreatic Neuroendocrine Tumors and Non-Functional Pancreatic Neuroendocrine Tumors. Neuroendocrinology. 2016;103(2):153-71.

74. Rindi G, Falconi M, Klersy C, Albarello L, Boninsegna L, Buchler MW, et al. TNM staging of neoplasms of the endocrine pancreas: results from a large international cohort study. J Natl Cancer Inst. 2012;104(10):764-77.

75. Basturk $\mathrm{O}$, Yang ZH, Tang $\mathrm{LH}$, Hruban RH, Adsay $\mathrm{V}$, McCall CM, et al. The High-grade (WHO G3) Pancreatic Neuroendocrine Tumor Category Is Morphologically and Biologically Heterogenous and Includes Both Well Differentiated and Poorly Differentiated Neoplasms. American Journal of Surgical Pathology. 
2015;39(5):683-90.

76. Solcia E, Klöppel G, Sobin LH. Histological Typing of Endocrine Tumours: Springer Science \& Business Media; 2000. $156 \mathrm{p}$.

77. Hammond EH, Yowell RL, Flinner RL. Neuroendocrine carcinomas: role of immunocytochemistry and electron microscopy. Hum Pathol. 1998;29(12):1367-71.

78. Yachida S, Vakiani E, White CM, Zhong Y, Saunders T, Morgan R, et al. Small cell and large cell neuroendocrine carcinomas of the pancreas are genetically similar and distinct from well-differentiated pancreatic neuroendocrine tumors. Am J Surg Pathol. 2012;36(2):173-84.

79. Sipos B, Sperveslage J, Anlauf M, Hoffmeister M, Henopp T, Buch S, et al. Glucagon cell hyperplasia and neoplasia with and without glucagon receptor mutations. J Clin Endocrinol Metab. 2015;100(5):E783-8.

80. Kloppel G, Anlauf M, Perren A, Sipos B. Hyperplasia to neoplasia sequence of duodenal and pancreatic neuroendocrine diseases and pseudohyperplasia of the PP-cells in the pancreas. Endocr Pathol. 2014;25(2):181-5.

81. Esposito I, Segler A, Steiger K, Kloppel G. Pathology, genetics and precursors of human and experimental pancreatic neoplasms: An update. Pancreatology. 2015;15(6):598-610. 82. Jiao Y, Shi C, Edil BH, de Wilde RF, Klimstra DS, Maitra A, et al. DAXX/ATRX, MEN1, and mTOR pathway genes are frequently altered in pancreatic neuroendocrine tumors. Science. 2011;331(6021):1199-203.

83. Lubensky IA, Debelenko LV, Zhuang Z, Emmert-Buck MR, Dong Q, Chandrasekharappa S, et al. Allelic deletions on chromosome $11 \mathrm{q} 13$ in multiple tumors from individual MEN1 patients. Cancer Res. 1996;56(22):5272-8.

84. Görtz B, Roth J, Krähenmann A, de Krijger RR,

Muletta-Feurer S, Rütimann K, et al. Mutations and allelic deletions of the MEN1 gene are associated with a subset of sporadic endocrine pancreatic and neuroendocrine tumors and not restricted to foregut neoplasms. Am J Pathol. 1999;154(2):429-36.

85. Heaphy CM, de Wilde RF, Jiao Y, Klein AP, Edil BH, Shi C, et al. Altered telomeres in tumors with ATRX and DAXX mutations. Science. 2011;333(6041):425.

86. Yuan F, Shi M, Ji J, Shi H, Zhou C, Yu Y, et al. KRAS and DAXX/ATRX gene mutations are correlated with the clinicopathological features, advanced diseases, and poor prognosis in Chinese patients with pancreatic neuroendocrine tumors. Int J Biol Sci. 2014;10(9):957-65.

87. Marinoni I, Kurrer AS, Vassella E, Dettmer M, Rudolph $T$, Banz V, et al. Loss of DAXX and ATRX are associated with chromosome instability and reduced survival of patients with pancreatic neuroendocrine tumors. Gastroenterology. 2014;146(2):453-60 e5.

88. Schmitt AM, Schmid S, Rudolph T, Anlauf M, Prinz C, Kloppel G, et al. VHL inactivation is an important pathway for the development of malignant sporadic pancreatic endocrine tumors. Endocr-Relat Cancer. 2009;16(4):1219-27.

89. Nikiforova MN, Nikiforov YE, Biddinger P, Gnepp $D R$, Grosembacher LA, Wajchenberg BL, et al. Frequent loss of heterozygosity at chromosome 3p14.2-3p21 in human pancreatic islet cell tumours. Clin Endocrinol. 1999;51(1):27-33.

90. Ohki R, Saito K, Chen Y, Kawase T, Hiraoka N,
Saigawa R, et al. PHLDA3 is a novel tumor suppressor of pancreatic neuroendocrine tumors. P Natl Acad Sci USA. 2014;111(23):E2404-E13.

91. Floridia G, Grilli G, Salvatore M, Pescucci C, Moore

PS, Scarpa A, et al. Chromosomal alterations detected by comparative genomic hybridization in nonfunctioning endocrine pancreatic tumors. Cancer Genet Cytogenet. 2005;156(1):23-30.

92. Hessman O, Skogseid B, Westin G, Akerstrom

G. Multiple allelic deletions and intratumoral genetic heterogeneity in men1 pancreatic tumors. J Clin Endocrinol Metab. 2001;86(3):1355-61.

93. Hessman $O$, Lindberg $D$, Einarsson $A$, Lillhager $P$, Carling T, Grimelius L, et al. Genetic alterations on 3p, 11q13, and $18 q$ in nonfamilial and MEN 1-associated pancreatic endocrine tumors. Genes Chromosomes Cancer. 1999;26(3):258-64.

94. Perren A, Anlauf M, Henopp T, Rudolph T, Schmitt A, Raffel A, et al. Multiple endocrine neoplasia type 1 (MEN1): loss of one MEN1 allele in tumors and monohormonal endocrine cell clusters but not in islet hyperplasia of the pancreas. J Clin Endocrinol Metab. 2007:92(3):1118-28.

95. de Wilde RF, Heaphy CM, Maitra A, Meeker AK, Edil $\mathrm{BH}$, Wolfgang $\mathrm{CL}$, et al. Loss of ATRX or DAXX expression and concomitant acquisition of the alternative lengthening of telomeres phenotype are late events in a small subset of MEN-1 syndrome pancreatic neuroendocrine tumors. Mod Pathol. 2012;25(7):10339.

96. Stefanoli M, La Rosa S, Sahnane N, Romualdi C,

Pastorino R, Marando A, et al. Prognostic relevance of aberrant

DNA methylation in $\mathrm{g} 1$ and $\mathrm{g} 2$ pancreatic neuroendocrine tumors. Neuroendocrinology. 2014;100(1):26-34.

97. House MG, Herman JG, Guo MZ, Hooker CM, Schulick RD, Lillemoe KD, et al. Aberrant hypermethylation of tumor suppressor genes in pancreatic endocrine neoplasms. Ann Surg. 2003;238(3):423-31; discussion 31-2.

98. Malpeli G, Amato E, Dandrea M, Fumagalli C, Debattisti V, Boninsegna L, et al. Methylation-associated down-regulation of RASSF1A and up-regulation of RASSF1C in pancreatic endocrine tumors. BMC Cancer. 2011;11(1):351.

99. Estrabaud E, Lassot I, Blot G, Le Rouzic E, Tanchou V, Quemeneur E, et al. RASSF1C, an isoform of the tumor suppressor RASSF1A, promotes the accumulation of beta-catenin by interacting with betaTrCP. Cancer Res. 2007;67(3):1054-61.

100. Ram RR, Mendiratta S, Bodemann BO, Torres MJ, Eskiocak $U$, White MA. RASSF1A inactivation unleashes a tumor suppressor/oncogene cascade with context-dependent consequences on cell cycle progression. Mol Cell Biol. 2014;34(12):2350-8.

101. Roldo C, Missiaglia E, Hagan JP, Falconi M,

Capelli P, Bersani S, et al. MicroRNA expression abnormalities in pancreatic endocrine and acinar tumors are associated with distinctive pathologic features and clinical behavior. J Clin Oncol. 2006;24(29):4677-84.

102. Thorns C, Schurmann C, Gebauer N, Wallaschofski H, Kumpers C, Bernard V, et al. Global microRNA profiling of pancreatic neuroendocrine neoplasias. Anticancer Res. 2014;34(5):224954.

103. Maitra A, Hansel DE, Argani P, Ashfaq R, Rahman A,

Naji A, et al. Global expression analysis of well-differentiated pan creatic endocrine neoplasms using oligonucleotide microarrays. 
Clinical Cancer Research. 2003;9(16):5988-95

104. Speisky D, Duces A, Bieche I, Rebours V, Hammel P, Sauvanet A, et al. Molecular profiling of pancreatic neuroendocrine tumors in sporadic and Von Hippel-Lindau patients. Clin Cancer Res. 2012;18(10):2838-49.

105. Raymond E, Dahan L, Raoul JL, Bang YJ, Borbath I, Lombard-Bohas $C$, et al. Sunitinib malate for the treatment of pancreatic neuroendocrine tumors. N Engl J Med. 2011;364(6):50113.

106. Vinik Al, Raymond E. Pancreatic neuroendocrine tumors: approach to treatment with focus on sunitinib. Therap Adv Gastroenterol. 2013;6(5):396-411.

107. Pea A, Hruban RH, Wood LD. Genetics of pancreatic neuroendocrine tumors: implications for the clinic. Expert Rev Gastroenterol Hepatol. 2015;9(11):1407-19.

108. Yao JC, Shah MH, Ito T, Bohas CL, Wolin EM, Van Cut sem $E$, et al. Everolimus for advanced pancreatic neuroendocrine tumors. N Engl J Med. 2011;364(6):514-23.

109. Law JK, Ahmed A, Singh VK, Akshintala VS, Olson MT, Raman SP, et al. A systematic review of solid-pseudopapillary neoplasms: are these rare lesions? Pancreas. 2014;43(3):331-7.

110. Notohara K, Hamazaki S, Tsukayama C, Nakamoto S, Kawabata K, Mizobuchi K, et al. Solid-pseudopapillary tumor of the pancreas: immunohistochemical localization of neuroendocrine markers and CD10. Am J Surg Pathol. 2000;24(10):1361-71. 111. Miettinen M, Partanen S, Fraki O, Kivilaakso E. Papillary cystic tumor of the pancreas. An analysis of cellular differentiation by electron microscopy and immunohistochemistry. Am J Surg Pathol. 1987;11(11):855-65.

112. El-Bahrawy MA, Rowan A, Horncastle D, Tomlinson I, Theis BA, Russell RC, et al. E-cadherin/catenin complex status in solid pseudopapillary tumor of the pancreas. Am J Surg Pathol. 2008;32(1):1-7.

113. Guo Y, Yuan F, Deng H, Wang HF, Jin XL, Xiao JC. Paranuclear dot-like immunostaining for CD99: a unique staining pattern for diagnosing solid-pseudopapillary neoplasm of the pancreas. Am J Surg Pathol. 2011;35(6):799-806.

114. Singhi AD, Lilo M, Hruban RH, Cressman KL, Fuhrer K, Seethala RR. Overexpression of lymphoid enhancer-binding factor 1 (LEF1) in solid-pseudopapillary neoplasms of the pancreas. Mod Pathol. 2014;27(10):1355-63.

115. Tanaka Y, Kato K, Notohara K, Hojo H, ljiri R, Miyake T, et al. Frequent beta-catenin mutation and cytoplasmic/nuclear accumulation in pancreatic solid-pseudopapillary neoplasm. Cancer Res. 2001;61(23):8401-4.

116. Abraham SC, Klimstra DS, Wilentz RE, Yeo CJ, Conlon K, Brennan M, et al. Solid-pseudopapillary tumors of the pancreas are genetically distinct from pancreatic ductal adenocarcinomas and almost always harbor beta-catenin mutations. American Journal of Pathology. 2002;160(4):1361-9.

117. Ruo L, Coit DG, Brennan MF, Guillem JG. Long term follow-up of patients with familial adenomatous polyposis undergoing pancreaticoduodenal surgery. J Gastrointest Surg. 2002;6(5):671-5.

118. Inoue T, Nishi Y, Okumura F, Mizushima T, Nishie H, Iwasaki $\mathrm{H}$, et al. Solid pseudopapillary neoplasm of the pancreas associated with familial adenomatous polyposis. Intern Med. 2015;54(11):1349-55.
119. Tognarini I, Tonelli F, Nesi G, Martineti V, Galli G, Gozzini A, et al. In vitro effects of oestrogens, antioestrogens and SERMs on pancreatic solid pseudopapillary neoplasm-derived primary cell culture. Cell Oncol. 2010;32(5-6):331-43.

120. Muller-Hocker J, Zietz C, Sendelhofert A. Deregulated expression of cell cycle-associated proteins in solid pseudopapillary tumor of the pancreas. Modern Pathol. 2001;14(2):47-53.

121. Tiemann K, Heitling U, Kosmahl M, Kloppel G. Solid pseudopapillary neoplasms of the pancreas show an interruption of the Wnt-signaling pathway and express gene products of 11q. Modern Pathol. 2007;20(9):955-60.

122. Park M, Lim JS, Lee HJ, Na K, Lee MJ, Kang CM, et al. Distinct Protein Expression Profiles of Solid-Pseudopapillary Neoplasms of the Pancreas. J Proteome Res. 2015;14(8):3007-14. 123. Park M, Kim M, Hwang D, Park M, Kim WK, Kim SK, et al. Characterization of gene expression and activated signaling pathways in solid-pseudopapillary neoplasm of pancreas. Mod Pathol. 2014;27(4):580-93.

124. Cavard C, Audebourg A, Letourneur F, Audard V, Beuvon F, Cagnard N, et al. Gene expression profiling provides insights into the pathways involved in solid pseudopapillary neoplasm of the pancreas. Journal of Pathology. 2009;218(2):201-9.

125. Seket B, Saurin JC, Scoazec JY, Partensky C. [Pancreatic acinar cell carcinoma in a patient with familial adenomatous polyposis]. Gastroenterol Clin Biol. 2003;27(8-9):818-20.

126. Lowery MA, Klimstra DS, Shia JR, Yu KH, Allen PJ, Brennan MF, et al. Acinar Cell Carcinoma of the Pancreas: New Genetic and Treatment Insights into a Rare Malignancy. Oncologist. 2011;16(12):1714-20.

127. de Wilde RF, Ottenhof NA, Jansen M, Morsink FH, de Leng WW, Offerhaus GJ, et al. Analysis of LKB1 mutations and other molecular alterations in pancreatic acinar cell carcinoma. Mod Pathol. 2011;24(9):1229-36.

128. Liu W, Shia J, Gonen M, Lowery MA, O'Reilly EM, Klimstra DS. DNA mismatch repair abnormalities in acinar cell carcinoma of the pancreas: frequency and clinical significance. Pancreas. 2014;43(8):1264-70.

129. Wisnoski NC, Townsend CM, Jr., Nealon WH, Freeman JL, Riall TS. 672 patients with acinar cell carcinoma of the pancreas: a population-based comparison to pancreatic adenocarcinoma. Surgery. 2008;144(2):141-8.

130. Cingolani N, Shaco-Levy R, Farruggio A, Klimstra DS, Rosai J. Alpha-fetoprotein production by pancreatic tumors exhibiting acinar cell differentiation: study of five cases, one arising in a mediastinal teratoma. Hum Pathol. 2000;31(8):938-44.

131. Ono J, Sakamoto H, Sakoda K, Yagi Y, Hagio S, Sato

$E$, et al. Acinar cell carcinoma of the pancreas with elevated serum alpha-fetoprotein. Int Surg. 1984;69(4):361-4.

132. Klimstra DS, Heffess CS, Oertel JE, Rosai J. Acinar cell carcinoma of the pancreas. A clinicopathologic study of 28 cases. Am J Surg Pathol. 1992;16(9):815-37.

133. La Rosa S, Franzi F, Marchet S, Finzi G, Clerici M, Vigetti $D$, et al. The monoclonal anti-BCL10 antibody (clone 331.1) is a sensitive and specific marker of pancreatic acinar cell carcinoma and pancreatic metaplasia. Virchows Arch. 2009;454(2):133-42. 134. Hosoda W, Sasaki E, Murakami Y, Yamao K, Shimizu Y, Yatabe Y. BCL10 as a useful marker for pancreatic acinar cell carcinoma, especially using endoscopic ultrasound cytology 
specimens. Pathol Int. 2013;63(3):176-82.

135. Yasumoto M, Hamabashiri M, Akiba J, Ogasawara S, Naito Y, Taira T, et al. The utility of a novel antibody in the pathological diagnosis of pancreatic acinar cell carcinoma. J Clin Pathol. 2012;65(4):327-32.

136. Abraham SC, Wu TT, Hruban RH, Lee JH, Yeo CJ, Conlon K, et al. Genetic and immunohistochemical analysis of pancreatic acinar cell carcinoma: frequent allelic loss on chromosome $11 \mathrm{p}$ and alterations in the APC/beta-catenin pathway. Am J Pathol. 2002;160(3):953-62.

137. Jiao Y, Yonescu R, Offerhaus GJ, Klimstra DS, Maitra A, Eshleman JR, et al. Whole-exome sequencing of pancreatic neoplasms with acinar differentiation. J Pathol. 2014;232(4):42835.

138. Rigaud G, Moore PS, Zamboni G, Orlandini S, Taruscio D, Paradisi S, et al. Allelotype of pancreatic acinar cell carcinoma. Int J Cancer. 2000;88(5):772-7.

139. La Rosa S, Sessa F, Capella C. Acinar Cell Carcinoma of the Pancreas: Overview of Clinicopathologic Features and Insights into the Molecular Pathology. Front Med (Lausanne). 2015;2(Suppl 2):41.

140. Dewald GW, Smyrk TC, Thorland EC, McWilliams RR, Van Dyke DL, Keefe JG, et al. Fluorescence in situ hybridization to visualize genetic abnormalities in interphase cells of acinar cell carcinoma, ductal adenocarcinoma, and islet cell carcinoma of the pancreas. Mayo Clin Proc. 2009;84(9):801-10.

141. Chmielecki J, Hutchinson KE, Frampton GM, Chalmers ZR, Johnson A, Shi C, et al. Comprehensive genomic profiling of pancreatic acinar cell carcinomas identifies recurrent RAF fusions and frequent inactivation of DNA repair genes. Cancer Discov. 2014;4(12):1398-405.

142. Furlan D, Sahnane N, Bernasconi B, Frattini M, Tibiletti MG, Molinari F, et al. APC alterations are frequently involved in the pathogenesis of acinar cell carcinoma of the pancreas, mainly through gene loss and promoter hypermethylation. Virchows Archiv. 2014;464(5):553-64.

143. Guo M, Jia Y, Yu Z, House MG, Esteller M, Brock MV et al. Epigenetic changes associated with neoplasms of the exocrine and endocrine pancreas. Discov Med. 2014;17(92):67-73. 144. Dhebri AR, Connor S, Campbell F, Ghaneh P, Sutton R, Neoptolemos JP. Diagnosis, treatment and outcome of pancreatoblastoma. Pancreatology. 2004;4(5):441-51; discussion 52-3. 145. Salman B, Brat G, Yoon YS, Hruban RH, Singhi AD,

Fishman EK, et al. The diagnosis and surgical treatment of pancreatoblastoma in adults: a case series and review of the literature. J Gastrointest Surg. 2013;17(12):2153-61.

146. Weksberg R, Shuman C, Beckwith JB. Beckwith-Wiedemann syndrome. Eur J Hum Genet. 2010;18(1):8-14.

147. Abraham SC, Wu TT, Klimstra DS, Finn LS, Lee JH, Yeo CJ, et al. Distinctive molecular genetic alterations in sporadic and familial adenomatous polyposis-associated pancreatoblastomas : frequent alterations in the APC/beta-catenin pathway and chromosome 11p. Am J Pathol. 2001;159(5):1619-27.

148. Maher ER, Reik W. Beckwith-Wiedemann syndrome: imprinting in clusters revisited. J Clin Invest. 2000;105(3):247-52. 149. Wong IH, Chan J, Wong J, Tam PK. Ubiquitous aberrant RASSF1A promoter methylation in childhood neoplasia. Clin Cancer Res. 2004;10(3):994-1002.

150. Honda S, Okada T, Miyagi H, Minato M, Suzuki H, Taketomi A. Spontaneous rupture of an advanced pancreatoblastoma: Aberrant RASSF1A methylation and CTNNB1 mutation as molecular genetic markers. J Pediatr Surg. 2013;48(4):E29-E32. 151. Ghiorzo P. Genetic predisposition to pancreatic cancer. World J Gastroenterol. 2014;20(31):10778-89. 


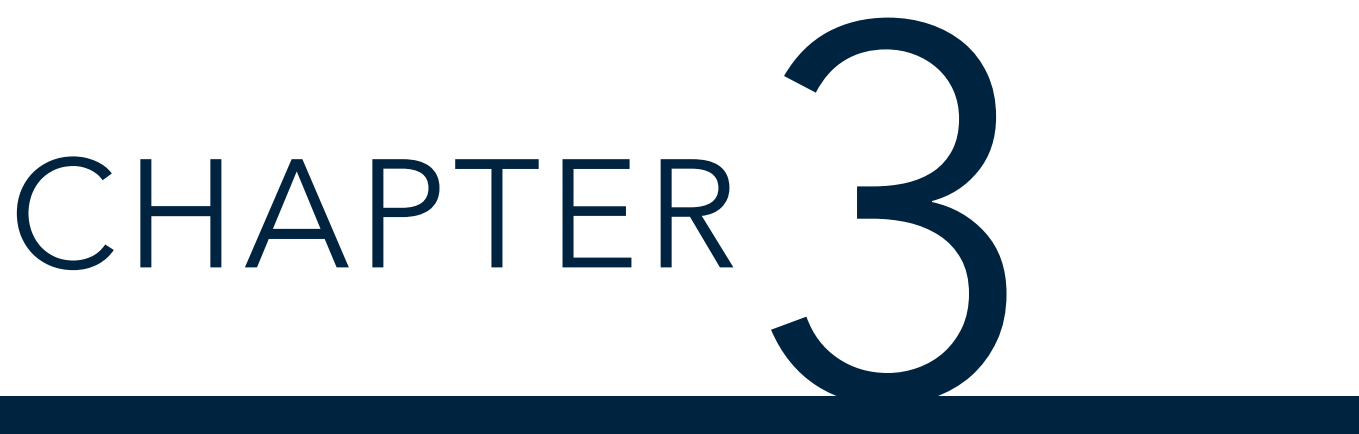

Aberrant Menin expression is an early event in pancreatic neuroendocrine tumorigenesis

Wenzel M. Hackeng, Lodewijk A. A. Brosens, Katherine E. Poruk, Michaël Noë, Waki Hosoda, Justin S. Poling, Anthony Rizzo, Martha Campbell-Thompson, Mark A. Atkinson, Björn Konukiewitz, Günter Klöppel, Christopher M. Heaphy, Alan K. Meeker, Laura D. Wood 


\section{ABSTRACT}

Pancreatic neuroendocrine tumors (PanNETs) are the second most common pancreatic malignancy and cause significant morbidity and mortality. Neuroendocrine microadenomas have been proposed as a potential precursor lesion for sporadic PanNETs. In this study, we applied telomere-specific fluorescent in situ hybridization (FISH) to a series of well-characterized sporadic neuroendocrine microadenomas and investigated the prevalence of alterations in known PanNET driver genes (MEN1 and $A T R X / D A X X)$ in these same tumors using immunohistochemistry for the encoded proteins. We identified aberrant Menin expression in 14 of 19 (74\%) microadenomas, suggesting that alterations in Menin, at least a subset of which were likely due to somatic mutation, are early events in pancreatic neuroendocrine tumorigenesis. In contrast, none of the microadenomas met criteria for the alternative lengthening of telomeres phenotype (ALT) based on telomere FISH, a phenotype that is strongly correlated to ATRX or DAXX mutations. Two of 15 microadenomas (13\%) were noted to have very rare abnormal bright telomere foci on FISH, suggestive of early ALT, but these lesions did not show loss of ATRX or DAXX protein expression by immunohistochemistry. Overall, these data suggest that loss of Menin is an early event in pancreatic neuroendocrine tumorigenesis and ATRX/DAXX loss and ALT are relatively late events. 


\section{INTRODUCTION}

Pancreatic neuroendocrine tumors (PanNET) are the second most common malignant neoplasm in the pancreas after ductal adenocarcinoma (1). Although usually less aggressive than pancreatic ductal adenocarcinoma, the prognosis of PanNETs is still poor, with overall 5 year survival averaging only 40\% (2). Recent studies have elucidated the mutational signature of sporadic PanNET; the driver genes in pancreatic neuroendocrine tumorigenesis are now known, but the timing of these alterations and their functional roles remain to be clarified. As neuroendocrine lesions are fairly common incidental findings (3), it is important to clarify which alterations account for the initiation of early lesions and which fuel the progression to more clinically aggressive neoplasms.

PanNETs usually arise sporadically but can also occur in patients with inherited cancer predisposition syndromes such as multiple endocrine neoplasia type 1 (MEN-1). Patients with MEN-1 syndrome have a germline mutation in the tumor suppressor gene MEN1 and have a $70 \%$ lifetime risk in developing PanNET (4). Somatic loss of the remaining wildtype allele of MEN1 (11q13) has been reported in up to 100\% of tumors in these syndromic patients (5-7). In patients without the inherited MEN-1 syndrome, $44 \%$ of sporadic PanNETs have somatic mutations in the MEN1 gene (8), and $19-44 \%$ of sporadic PanNETs have loss of heterozygosity at the MEN1 locus $(5,9)$.

In addition to frequent somatic mutations in MEN1, recent whole-exome and targeted sequencing studies have shown that $43 \%$ of sporadic PanNETs have somatic inactivating mutations in ATRX or DAXX (8). These genes encode members of a chromatin-remodeling complex which incorporates the histone variant H3.3 into telomeric DNA. Mutations in ATRX and DAXX are associated with the alternative lengthening of telomeres (ALT) phenotype, a telomerase independent mechanism of telomere maintenance (10). Recent studies have shown that mutations in ATRX and DAXX (with activation of ALT) in PanNETs correlate with larger tumor size, advanced tumor stage, and chromosomal instability, suggesting that these genetic alterations are late events in sporadic PanNET progression and/or that they confer a more aggressive phenotype (11).

Microadenomas of the pancreas are histologically similar to PanNETs but are by definition smaller than $5 \mathrm{~mm}$ (12). Although not universally accepted, some investigators believe that microadenomas are precursors to larger PanNETs. To date, early tumorigenesis in sporadic 
PanNETs remains understudied, while PanNET development has been well studied in MEN-1 patients. MEN-1 patients have many microadenomas, and it has been shown that $100 \%$ of microadenomas in these patients have allelic loss of the MEN1 locus (which does not occur in surrounding ducts, acinar tissue, and hyperplastic islets) (6, 13). Because of these genetic alterations, as well as the high risk of PanNET development in these patients, microadenomas are considered precursors to PanNETs in MEN-1 patients $(14,15)$. In contrast to early-occurring MEN1 alterations, ATRX and DAXX expression was reported to be retained in all MEN1-associated microadenomas, demonstrating that ATRX and DAXX loss, and therefore ALT, are also likely late alterations in PanNET tumorigenesis in MEN-1 patients (16).

Sporadic microadenomas provide insights into the early tumorigenesis of sporadic PanNETs, distinguishing early from late-occurring genetic alterations. In this study we analyzed 19 well-characterized sporadic microadenomas with fluorescence in situ hybridization (FISH) and immunohistochemistry (IHC) to analyze the alternative lengthening of telomeres (ALT) phenotype, ATRX and DAXX expression, and Menin expression $(10,17)$.

\section{MATERIALS AND METHODS}

\section{Patients and tissue microadenomas}

The study was approved by the Institutional Review Board of The John Hopkins Hospital and was exempt from the Institutional Review Board of the University of Florida. Microadenomas for this study were identified from two sources. First, transplantation-suitable pancreata were recovered from organ donors and processed with identification of non-syndromic microadenomas in 4 donors as previously described (18). Second, additional cases of sporadic microadenoma were identified in surgical specimens from the pathology archives of The Johns Hopkins Hospital. For all cases the following exclusion criteria were used: lesion size $>5 \mathrm{~mm}$, synchronous PanNET, and MEN-1 and VHL syndrome. In total 19 cases were included in the study, including 4 cases from organ donors and 15 cases from surgically resected pancreata. Eight 5 um sections were cut onto plus slides (Cardinal Health, OH, USA) for IHC. Before and after cutting plus slides, a hematoxylin and eosin-stained (H\&E) slide was made to confirm the presence of microadenoma on slides for IHC. All H\&E slides were reviewed by an expert in pancreatic pathology to confirm the diagnosis of microadenoma.

PanNETs for validation of the Menin antibody were taken from a previously sequenced group of PanNETs by Jiao et al. (8). Six cases with homozygous or heterozygous mutations 
in MEN1 and 4 with wildtype MEN1 were selected to validate the antibody (Table 1). All cases were well-differentiated. Two 5um sections were cut on plus slides (Cardinal Health) for IHC.

\section{Immunohistochemistry}

Menin: Paraffin was cleared using a heatblock at $70^{\circ} \mathrm{C}$ for 30 seconds, deparaffinized for 2 minutes in xylene, rehydrated in 2 minutes in 100\% EtOH, 2 minutes 95\% EtOH and 2 minutes $70 \% \mathrm{EtOH}$. Slides were placed in a preheated Target Retrieval Solution (1:10, Dako, CA, USA) in a vegetable steamer for 30 minutes and then washed twice with wash buffer ( $1: 10$, Dako). After 20 minutes cooling at room temperature, slides were incubated in $0.2 \%$ triton TBS for 5 minutes. Endogenous enzyme activity was blocked for 10 minutes in the dark (Dual Endogenous Enzyme Block, Dako) and primary antibody anti-menin (1:1000 dilution, catalog\# A300-105A, lot 5, Bethyl, TX, USA) was applied in 10\% FBS in TBS for 2 hours at room temperature. Slides were incubated for 30 minutes with horseradish peroxidase reagent (Labeled Polymer - Dako REAL EnVision-HRP, Rabbit-Mouse, Dako) at room temperature. DAB staining (Dako) was done according to protocol for 10 minutes, and slides were counterstained with hematoxylin. Slides were dehydrated in alcohol and xylene, and mounted with a coverglass. Assessment of Menin expression was independently performed by four pathologists blinded to mutation status and classified in 4 categories: 0 (absent), 1 (weak), 2 (moderate), 3 (strong), with separate scores for nucleus and cytoplasm. As an internal control, the labeling in each microadenoma was compared to the labeling in a nearby Islet, in which moderate nuclear labeling and weak cytoplasmic labeling were expected. Specifically, weak nuclear labeling was defined as labeling of less intensity than nearby islets, while moderate nuclear labeling was of equal intensity to nearby islets, and strong nuclear labeling was of greater intensity than nearby islets. Nuclear labeling of 0 or 1 was considered aberrant. For cytoplasmic labeling, weak cytoplasmic labeling was of equal intensity to nearby islets, while moderate cytoplasmic labeling was somewhat more intense than nearby islets, and strong cytoplasmic labeling was much more intense than nearby islets. Cytoplasmic labeling of 2 or 3 was considered aberrant. A consensus Menin status was then determined for each case, requiring agreement of three pathologists to call the labeling "aberrant". We required agreement of three pathologists, rather than unanimous agreement of four pathologists, because this correlated better in our PanNETs with known MEN1 mutation status.

ATRX/DAXX: Immunolabeling for the ATRX and DAXX proteins was performed on formalin- 
fixed, paraffin embedded sections as previously described (8). Briefly, heat-induced antigen retrieval was performed in a steamer using citrate buffer (catalog\# H-3300, Vector Laboratories, CA, USA) for 30 minutes. Endogenous peroxidase was blocked (catalog\# S2003, Dako) and serial sections were then incubated with primary antibody; anti-ATRX (1:400 dilution; catalog\# HPA001906, lot R00473, Sigma-Aldrich, MO, USA) or anti-DAXX (1:150 dilution; catalog\# HPA008736, lot A39105, Sigma-Aldrich) for 1 hour at room temperature. The primary antibodies were detected by 30 minute incubation with HRPlabeled secondary antibody (catalog\# PV6119, Leica Microsystems, IL, USA) followed by detection with 3,3'-Diaminobenzidine (Sigma Aldrich), counterstaining with Harris hematoxylin, rehydration and mounting. Only nuclear labeling of either protein was evaluated. The immunolabeled PanNET slides were assessed and scored by AKM and were designated as retained or lost. Internal controls included islets of Langerhans and ductal cells, which demonstrated strong nuclear immunolabeling for both ATRX and DAXX - labeling of these controls was required for evaluation of the section.

\section{Telomere-specific FISH and microscopy}

Combined telomere-specific FISH and immunofluorescence labeling for ATRX, DAXX and PML was conducted as previously described (19). Briefly, deparaffinized slides were hydrated, steamed for 20 minutes in citrate buffer (catalog\# H-3300; Vector Laboratories), dehydrated and hybridized with a Cy3-labeled peptide nucleic acid (PNA) probe complementary to the human telomere repeat sequence ([N-terminus to C-terminus] CCCTAACCCTAACCCTAA). As a positive control for hybridization efficiency, a FITC-labeled PNA probe having specificity for human centromeric DNA repeats (ATTCGTTGGAAACGGGA; CENP-B binding sequence) was also included in the hybridization solution (20). Following post-hybridization washes, the desired primary antibody was applied (anti-ATRX, as described above; anti-DAXX, as described above; anti-PML antibody, 45 minute incubation at 1:100 dilution; catalog\# PGM3, Dako), followed by application of species-appropriate Alexa 488 fluorescent secondary antibody (Molecular Probes Cat.\# A-11034 and A-11001) and nuclear counterstaining with DAPI. Slides were imaged with a Nikon 50i epifluorescence microscope equipped with X-Cite series 120 illuminator (EXFO Photonics Solutions Inc., CA, USA) and appropriate fluorescence excitation/emission filters. Grayscale images were captured with an attached Photometrics CoolsnapEZ digital camera, pseudo-colored, and merged using Nikon NES Elements software. Quantification from the digital images was conducted using Telometer, a custom software plugin created for the open source image analysis program ImageJ, freely available for download (http://bui2.win.ad.jhu.edu/telometer/). 

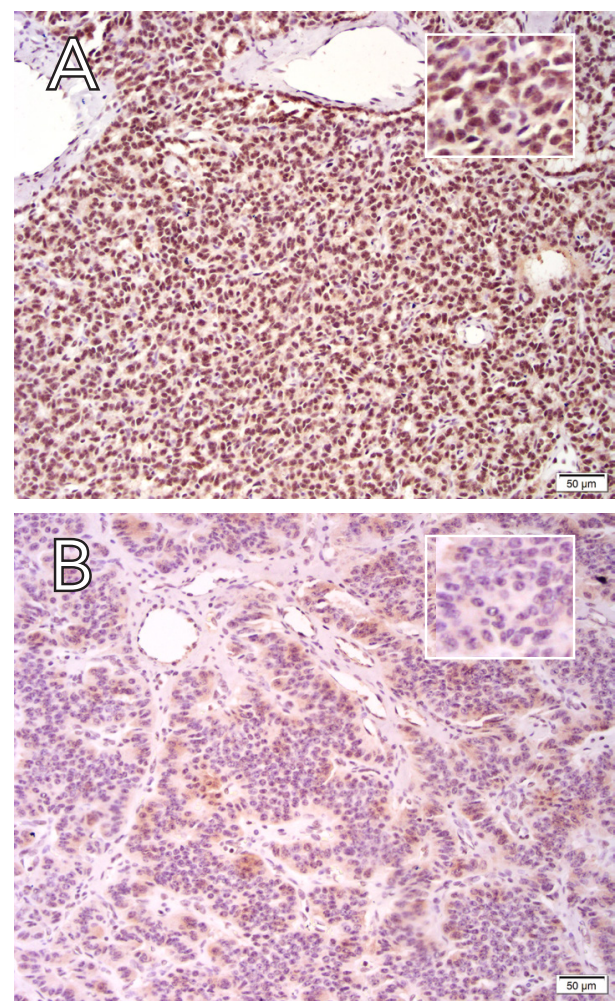

Figure 1. Immunohistochemistry for Menin in PanNETs with known MEN1 mutation status. A, MEN1-wildtype PanNET. Menin IHC and hematoxylin counterstain shows normal nuclear Menin expression and normal cytoplasmic Menin expression. Magnification box for zoomed-in view on cells. B, MEN1-mutated PanNET. Menin IHC and hematoxylin counterstain shows aberrant nuclear Menin expression and normal cytoplasmic Menin expression. Magnification box for zoomed-in view on cells.

Table 1. Immunohistochemistry for Menin in PanNETs with known MEN1 mutation status

\begin{tabular}{llllll}
\hline Sample & Nuclear Labeling & Cytoplasmic Labeling & MEN1 Status & Mutation & Type of Mutation \\
\hline PEN49 & Normal & $?$ & WT & & \\
PEN79 & Normal & Normal & WT & & \\
PEN76 & Normal & Normal & WT & & \\
PEN104 & Aberrant & Normal & WT & & HOM FS \\
PEN39 & Aberrant & Normal & MUT & c.159_165delCATCCCT & HOM NS \\
PEN29 & Aberrant & Aberrant & MUT & c.88G >T & c.79_95delCTGGGCCGAGAGGAGCC HET FS \\
PEN77 & Aberrant & Normal & MUT & c.249_252delGTCT & HET FS \\
PEN93 & Aberrant & $?$ & MUT & c.50_53delACGA & HOM FS \\
PEN10 & Aberrant & Not Scored & MUT & HET NS \\
PEN31 & Aberrant & Not Scored & MUT & c.1643C $>$ A & \\
\hline
\end{tabular}

Abbreviations: WT, wildtype; MUT, mutant; HOM, homozygous; HET, heterozygous; FS, frameshift; NS, nonsense 

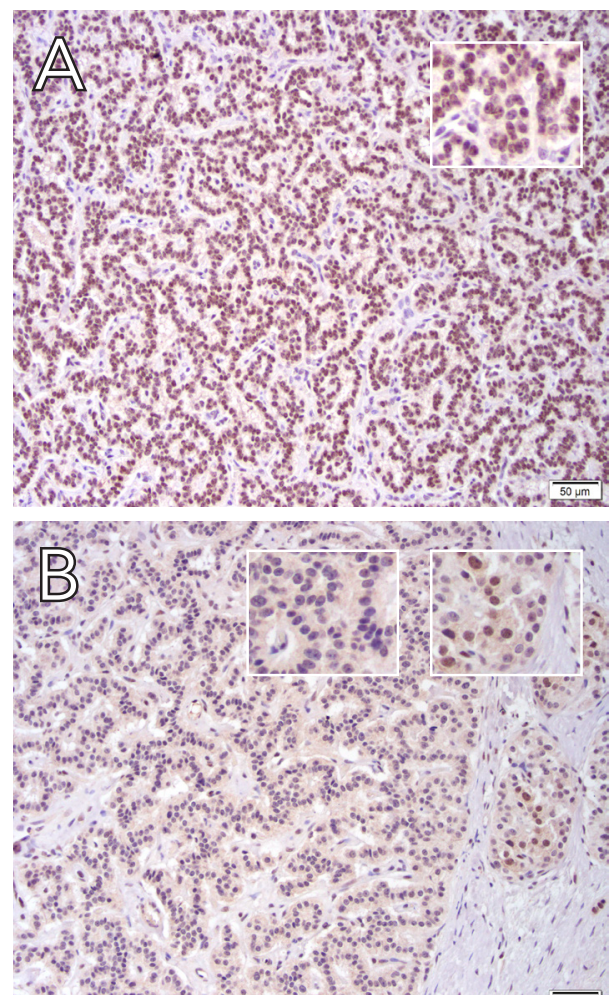

Figure 2. Immunohistochemistry for Menin in microadenomas. A, microadenoma with normal Menin labeling. Menin $\mathrm{IHC}$ and hematoxylin counterstain shows normal nuclear Menin expression and normal cytoplasmic Menin expression. B, microadenoma with aberrant Menin labeling. Menin IHC and hematoxylin counterstain shows aberrant nuclear Menin expression and normal cytoplasmic Menin expression. Note the nearby ducts with moderate nuclear Menin expression and weak cytoplasmic Menin expression. Magnification boxes for zoomed-in view on cells seen in the microadenoma and the nearby normal islets for comparison of staining.
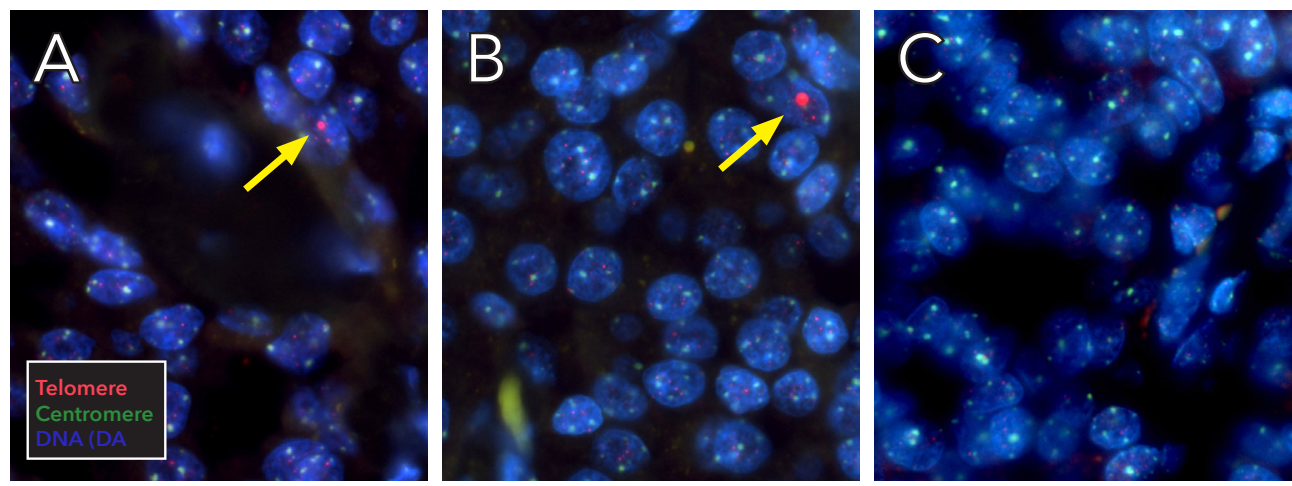

Figure 3. Telomere-specific FISH in microadenomas. A, representative image of microadenoma with single bright telomere focus (yellow arrow). B, representative image of same microadenoma in A with separate single bright telomere focus (yellow arrow). C, representative image of different microadenoma from same patient in A \& B showing diffuse short telomeres but no bright telomere foci. 
Table 2. Immunohistochemistry for Menin and telomere FISH in microadenoma

\begin{tabular}{|c|c|c|c|c|c|c|c|c|c|}
\hline Patient & Age & Sex & $\begin{array}{l}\text { Reason for } \\
\text { pancreatic } \\
\text { resection }\end{array}$ & Sample & $\begin{array}{l}\text { Size } \\
(\mathrm{mm})\end{array}$ & $\begin{array}{l}\text { Nuclear Menin } \\
\text { Labeling }\end{array}$ & $\begin{array}{l}\text { Cytoplasmic } \\
\text { Menin Labeling } \\
\end{array}$ & Telomere length & $\begin{array}{l}\text { Bright telomere } \\
\text { foci? }\end{array}$ \\
\hline Patient 1 & 51 & $M$ & PDAC & MA1 & 2 & Aberrant & Normal & Normal & No \\
\hline Patient 2 & 78 & M & PDAC & MA2 & 2 & Normal & Normal & Normal & No \\
\hline Patient 3 & 67 & M & PDAC & MA3 & 5 & Aberrant & Normal & Not Done & Not Done \\
\hline Patient 4 & 70 & M & SCA & MA4 & 4 & Normal & Normal & Short & No \\
\hline Patient 5 & 36 & $\mathrm{~F}$ & SCA & MA5 & 3 & Aberrant & Normal & Normal & No \\
\hline Patient 6 & 85 & M & PDAC & MA6 & 2 & Normal & Normal & Normal & No \\
\hline Patient 7 & 64 & M & PDAC & MA7 & 2 & Aberrant & Normal & Short & Yes \\
\hline Patient 8 & 49 & M & Pancreatitis & MA8 & 2 & Aberrant & Normal & Short & No \\
\hline Patient 8 & 49 & M & Pancreatitis & MA9 & 2 & Aberrant & Normal & Short & No \\
\hline Patient 9 & 77 & $\mathrm{~F}$ & IPMN & MA10 & 4 & Normal & Normal & Normal & No \\
\hline Patient 10 & 43 & M & IPMN & MA11 & 3 & Aberrant & Aberrant & Short & No \\
\hline Patient 11 & 68 & M & IPMN & MA12 & 2 & Aberrant & Normal & Not Done & Not Done \\
\hline Patient 12 & 85 & $\mathrm{~F}$ & SCA & MA13 & 4 & Aberrant & Normal & Heterogeneous & Yes \\
\hline Patient 12 & 85 & $\mathrm{~F}$ & SCA & MA14 & 5 & Aberrant & Normal & Short & No \\
\hline Patient 13 & 84 & $\mathrm{~F}$ & PDAC & MA15 & 4 & Aberrant & Normal & Short & No \\
\hline Patient 14 & 59 & M & Organ Donor & MA16 & 2 & Aberrant & Normal & Not Done & Not Done \\
\hline Patient 15 & 57 & $\mathrm{~F}$ & Organ Donor & MA17 & 5 & Aberrant & Normal & Not Done & Not Done \\
\hline Patient 16 & 53 & M & Organ Donor & MA18 & 1 & Normal & Normal & Not Done & Not Done \\
\hline Patient 17 & 61 & $\mathrm{~F}$ & Organ Donor & MA19 & 4 & Aberrant & Normal & Not Done & Not Done \\
\hline
\end{tabular}

Abbreviations: MA, microadenoma; PDAC, pancreatic ductal adenocarcinoma; SCA, serous cystadenoma; IPMN, intraductal papillary mucinous neoplasm

The FISH and immunolabeled slides were assessed and scored by AKM. Large, ultra-bright telomere repeat DNA aggregates are unique to ALT-positive cell populations and are significantly larger and brighter than the FISH signals emanating from individual telomeres in the same cell population. In this study, PanNETs were classified as ALT-positive if they met the following criteria: (i) the presence of ultra-bright, intra-nuclear foci of telomere FISH signals, with integrated total signal intensities for individual foci being $>10$ fold that of the per cell mean integrated signal intensities for all telomeric signals in individual benign stromal cells within the same case; (ii) $\geq 1 \%$ of neoplastic cells displaying ALT-associated telomeric DNA foci. Tumor samples lacking ALT-associated telomeric foci, or falling below the $1 \%$ of focus-positive call threshold, were considered ALT-negative. In addition, telomere length was qualitatively assessed. Fluorescence signal intensities from telomere-specific FISH are linearly related to the length of the telomere repeat DNA target (19). Telomeres of cells within neoplastic lesions were directly compared by visual inspection to those in normal-appearing, benign stromal cells and/or epithelial cells in the surrounding tissue. From this qualitative assessment, telomere lengths were scored as being either short, normal, or heterogeneous.

\section{Statistics}

Fisher's exact test was used to correlate aberrant labeling with parameters. 


\section{RESULTS}

\section{Immunohistochemical analysis of Menin expression in PanNETs with known MEN1 mutations}

To validate the Menin antibody, immunohistochemistry for Menin was performed on PanNETs with known MEN1 mutation status (six mutant and four wild type), as determined in a previous whole exome sequencing study of PanNETs (Table 1) (10). Three of four MEN1 wild type PanNETs showed normal nuclear labeling (Figure 1A), while the remaining MEN1 wild type PanNET was agreed to have aberrant nuclear labeling by all four reviewers. All six MEN1 mutant PanNETs had aberrant nuclear labeling (Figure 1B). Only one MEN1 mutant PanNETs had aberrant cytoplasmic labeling - the remaining wild type and mutant PanNETs had normal cytoplasmic labeling. Absent or weak nuclear labeling correlated with any MEN 1 mutation ( $p=0.033$, Fisher's exact test). No significant correlation between cytoplasmic labeling and mutational status was found.

\section{Immunohistochemical analysis of Menin expression in microadenomas}

Immunohistochemistry for Menin was performed on all 19 microadenomas (Table 2). Normal moderate nuclear labeling was seen in 5 of 19 microadenomas (Figure 2A). Aberrant nuclear labeling (as compared to the internal control of nearby normal islets) was seen in the remaining 14 of 19 microadenomas (74\%) (Figure 2B). This sample set included two patients with two separate microadenomas. In both patients, both microadenomas showed aberrant nuclear Menin expression.

\section{Alternative lengthening of telomeres and immunohistochemical analysis of ATRX and DAXX expression in microadenomas}

Telomere-specific FISH was performed on 13 microadenomas. Approximately half of the microadenomas had abnormally short telomeres. No microadenomas met the criteria for ALT, but bright telomere foci were seen in 2 of 13 successfully stained microadenomas (15\%). In these microadenomas, bright telomere foci were noted only in very rare cells, far below the $1 \%$ cutoff for designation of the neoplastic lesion as ALT. In both cases bright telomere foci were seen exclusively in the microadenomas and not in the surrounding tissue. ATRX and DAXX immunohistochemistry was performed on the two microadenomas with bright telomere foci, and expression of both proteins was retained in both cases. In these two cases, we also performed concomitant immunohistochemistry for ATRX/DAXX and telomere FISH on the same tissue section - these studies did not reveal ATRX or DAXX loss even in the specific individual cells with bright telomere foci. 


\section{DISCUSSION}

In this study, a majority of neuroendocrine microadenomas (74\%) had aberrant nuclear expression of Menin. Decreased nuclear expression of Menin was exclusively seen in microadenomas and not in surrounding islets or ductal cells, strongly suggesting that loss of Menin expression is a specific feature of neoplastic neuroendocrine cells. Truncating mutations can eliminate the C-terminal nuclear localization signals of the Menin protein, thus causing decreased Menin nuclear localization $(21,22)$. Despite the correlation between loss of nuclear Menin expression and mutations in sporadic PanNETs, it is not possible to definitively conclude mutations are present in the microadenomas analyzed in this study. Corbo et al. used the same Menin antibody to correlate MEN1 mutation status and Menin expression; they also found a correlation between truncating mutations and absence of nuclear labeling (17). However, only $37 \%$ of PanNETs studied by Corbo et al. with weak or absent nuclear Menin expression had MEN1 mutations, showing that mechanisms other than mutation can cause altered nuclear Menin expression. It is also not possible to rule out mutations when normal expression of nuclear Menin is seen, as non-truncating MEN1 mutations might not influence Menin nuclear localization or the antibody's epitope (17).

Our data show an imperfect correlation between nuclear Menin labeling and MEN1 mutation in PanNETs with known MEN1 mutation status, with one wild-type PanNET showing aberrant nuclear labeling. Importantly, the aberrant nuclear labeling in this wild type PanNET was agreed upon by all four reviewing pathologists, suggesting that this represents Menin alteration by an alternative mechanism rather than an artifact of subjective interpretation of immunohistochemistry. Still, the strong correlation of MEN1 mutation with aberrant nuclear expression in our ten PanNETs with known mutation status points to the utility of Menin immunohistochemistry as a surrogate for MEN1 alterations.

Although our data demonstrate the overall utility of Menin immunohistochemistry, the lack of unanimous consensus on Menin status in the majority of microadenomas does highlight some subjectivity inherent to immunohistochemical assays. Still, unanimous consensus on aberrant nuclear Menin labeling occurred in seven microadenomas (37\%), demonstrating Menin alterations in these lesions even when using the strictest criteria for consensus. Taken together, these data suggest that Menin alterations are an early event in PanNET tumorigenesis, although the number of underlying MEN1 mutations remains to be assessed. 
In the present study, bright telomere foci were seen in 2 of 13 microadenomas (15\%) analyzed with telomere-specific FISH. These abnormal foci were highly specific for the two microadenomas compared to surrounding normal tissue (Figures $3 \mathrm{~A}$ and 3B). Additionally, in one of the two cases, there was an additional, spatially distinct microadenoma, which lacked cells with ALT-like telomeric foci and harbored an abnormally short telomere phenotype (Figure 3C). However, abnormal telomere foci were only seen in a small proportion of cells on the slide (1-3 cells per microadenoma) and therefore did not meet the ALT threshold of $\geq 1 \%$ of neoplastic cells. Notably, costaining for ATRX and DAXX by immunofluorescence did not reveal any loss of nuclear expression of these proteins in either microadenoma.

Alterations in ATRX and DAXX, either by mutation or loss of nuclear ATRX or DAXX expression, have been previously shown to be perfectly correlated with the ALT phenotype in advanced PanNETs (10). In contrast, another study showed an imperfect correlation in earlier stage PanNETs, with retention of ATRX/DAXX expression in 15\% of PanNETs with ALT and lack of ALT phenotype in 29\% of PanNETs with ATRX/DAXX loss (11). Retention of ATRX and DAXX expression in our microadenomas suggests either that the appearance of ALT-associated foci precedes the acquisition of inactivating mutations in ATRX or DAXX, or that these two microadenomas do not have the same type of ALT that has been previously described in PanNETs. This phenotype of very rare abnormal telomere foci could, therefore, represent a type of pre-ALT or a completely separate phenomenon. Nevertheless, the lack of ALT in sporadic microadenomas is strikingly different compared to sporadic PanNETs (approximately 60\% of which possess ALT (10)), suggesting that acquisition or selection of the ALT phenotype occurs after progression from microadenoma to sporadic PanNET. In addition, a recent study reports loss of DAXX expression in $29 \%$ of microadenomas and loss of ATRX expression in $57 \%$ of microadenomas. Although these authors did not investigate ALT in their cohort, their reported frequency of ATRX/DAXX loss is much higher the frequency of ALT in our study, possibly suggesting a de-coupling of these two molecular events in early neuroendocrine tumorigenesis (23).

In conclusion, using PanNET cases with known MEN1 mutation status, we confirm the correlation between mutation status and aberrant nuclear expression of Menin, as assessed by immunohistochemistry. Aberrant Menin expression, likely due to MEN1 mutation in at least a subset of cases, is a key initiator in PanNET tumorigenesis, as Menin expression is altered in the majority of neuroendocrine microadenomas. Although none of the microadenomas 
met the criteria for ALT, rare cells with abnormal telomere foci that have previously been shown to be tightly associated with ALT were observed in two microadenomas. This suggests the possibility of an incompletely developed early ALT phenotype in these cases, though lack of alteration in ATRX/DAXX expression in these neoplasms further complicates interpretation. Still, it is clear that (in contrast to Menin alterations) aberrant expression of ATRX/DAXX is a later event in PanNET tumorigenesis. Further studies of microadenomas could identify other initiators of sporadic PanNET development and could help differentiate the mutations that initiate tumor development from those that drive tumor progression.

\section{DISCLOSURE/CONFLICT OF INTEREST:}

Dr. Wood is a paid consultant for Personal Genome Diagnostics.

\section{ACKNOWLEDGEMENTS:}

This study was supported by NIH grants NIH CA62924 and CA126607-06A1; Sigma Beta Sorority; Dutch Cancer Society (KWF); Lisa Waller Hayes Foundation; Nijbakker Morra Foundation; Juvenile Diabetes Research Fund grants 6-2006-1440 and 25-2013-268; University of Florida College of Medicine Department of Pathology 


\section{REFERENCES}

1. Yao JC, Eisner MP, Leary C, Dagohoy C, Phan A, Rashid A, et al. Population-based study of islet cell carcinoma. Ann Surg Oncol. 2007;14(12):3492-500.

2. Ries L, Young JJ, Keel G, Eisner M, Lin Y, Horner M. Cancer Survival Among Adults: US Department of Health and Human Services, National Institutes of Health, National Cancer Institute.; 2007.

3. Kimura W, Kuroda A, Morioka Y. Clinical pathology of endocrine tumors of the pancreas. Analysis of autopsy cases. Dig Dis Sci. 1991;36(7):933-42.

4. Marini F, Falchetti A, Luzi E, Tonelli F, Luisa BM. Multiple Endocrine Neoplasia Type 1 (MEN1) Syndrome. 2008.

5. Lubensky IA, Debelenko LV, Zhuang Z, Emmert-Buck MR, Dong Q, Chandrasekharappa S, et al. Allelic deletions on chromosome 11q13 in multiple tumors from individual MEN1 patients. Cancer Res. 1996;56(22):5272-8.

6. Hessman O, Skogseid B, Westin G, Akerstrom

G. Multiple allelic deletions and intratumoral genetic heterogeneity in men 1 pancreatic tumors. J Clin Endocrinol Metab. 2001;86(3):1355-61.

7. Hessman $O$, Lindberg D, Einarsson A, Lillhager $P$, Carling T, Grimelius L, et al. Genetic alterations on 3p, 11 q13, and $18 q$ in nonfamilial and MEN 1-associated pancreatic endocrine tumors. Genes Chromosomes Cancer. 1999;26(3):258-64.

8. Jiao Y, Shi C, Edil BH, de Wilde RF, Klimstra DS, Maitra A, et al. DAXX/ATRX, MEN1, and mTOR pathway genes are frequently altered in pancreatic neuroendocrine tumors. Science. 2011;331(6021):1199-203.

9. Görtz B, Roth J, Krähenmann A, de Krijger RR,

Muletta-Feurer S, Rütimann K, et al. Mutations and allelic deletions of the MEN1 gene are associated with a subset of sporadic endocrine pancreatic and neuroendocrine tumors and not restricted to foregut neoplasms. Am J Pathol. 1999;154(2):429-36.

10. Heaphy $\mathrm{CM}$, de Wilde RF, Jiao Y, Klein AP, Edil BH, Shi $C$, et al. Altered telomeres in tumors with ATRX and DAXX mutations. Science. 2011;333(6041):425.

11. Marinoni I, Kurrer AS, Vassella E, Dettmer M, Rudolph T, Banz V, et al. Loss of DAXX and ATRX are associated with chromosome instability and reduced survival of patients with pancreatic neuroendocrine tumors. Gastroenterology. 2014;146(2):453-60 e5.

12. Klimstra DS, Modlin IR, Coppola D, Lloyd RV, Suster S. The pathologic classification of neuroendocrine tumors: a review of nomenclature, grading, and staging systems. Pancreas. 2010;39(6):707-12.

13. Perren A, Anlauf M, Henopp T, Rudolph T, Schmitt A, Raffel A, et al. Multiple endocrine neoplasia type 1 (MEN1): loss of one MEN1 allele in tumors and monohormonal endocrine cell clusters but not in islet hyperplasia of the pancreas. J Clin Endocrinol Metab. 2007;92(3):1118-28.

14. Esposito I, Segler A, Steiger K, Kloppel G. Pathology, genetics and precursors of human and experimental pancreatic neoplasms: An update. Pancreatology. 2015;15(6):598-610.

15. Kloppel G, Anlauf M, Perren A, Sipos B. Hyperplasia

to neoplasia sequence of duodenal and pancreatic neuroen-

docrine diseases and pseudohyperplasia of the PP-cells in the pancreas. Endocr Pathol. 2014;25(2):181-5.

16. de Wilde RF, Heaphy CM, Maitra A, Meeker AK, Edi $\mathrm{BH}$, Wolfgang $\mathrm{CL}$, et al. Loss of ATRX or DAXX expression and concomitant acquisition of the alternative lengthening of telomeres phenotype are late events in a small subset of MEN-1 syndrome pancreatic neuroendocrine tumors. Mod Pathol. 2012;25(7):10339.

17. Corbo V, Dalai I, Scardoni M, Barbi S, Beghelli S, Bersani S, et al. MEN1 in pancreatic endocrine tumors: analysis of gene and protein status in 169 sporadic neoplasms reveals alterations in the vast majority of cases. Endocr Relat Cancer. 2010;17(3):771-83.

18. Campbell-Thompson M, Wasserfall C, Kaddis J, Albanese-O'Neill A, Staeva T, Nierras C, et al. Network for Pancreatic Organ Donors with Diabetes (nPOD): developing a tissue biobank for type 1 diabetes. Diabetes Metab Res Rev. 2012;28(7):608-17. 19. Meeker AK, Gage WR, Hicks JL, Simon I, Coffman JR, Platz EA, et al. Telomere length assessment in human archival tissues: combined telomere fluorescence in situ hybridization and immunostaining. Am J Pathol. 2002;160(4):1259-68.

20. Chen C, Hong YK, Ontiveros SD, Egholm M, Strauss WM. Single base discrimination of CENP-B repeats on mouse and human Chromosomes with PNA-FISH. Mamm Genome. 1999;10(1):13-8.

21. Guru SC, Goldsmith PK, Burns AL, Marx SJ, Spiegel AM, Collins FS, et al. Menin, the product of the MEN1 gene, is a nuclear protein. P Natl Acad Sci USA. 1998;95(4):1630-4.

22. La P, Desmond A, Hou Z, Silva AC, Schnepp RW, Hua $X$. Tumor suppressor menin: the essential role of nuclear localization signal domains in coordinating gene expression. Oncogene. 2006;25(25):3537-46.

23. Hadano A, Hirabayashi K, Yamada M, Kawanishi A, Takanashi Y, Kawaguchi Y, et al. Molecular alterations in sporadic pancreatic neuroendocrine microadenomas. Pancreatology. $2016 ; 16(3): 411-5$ 


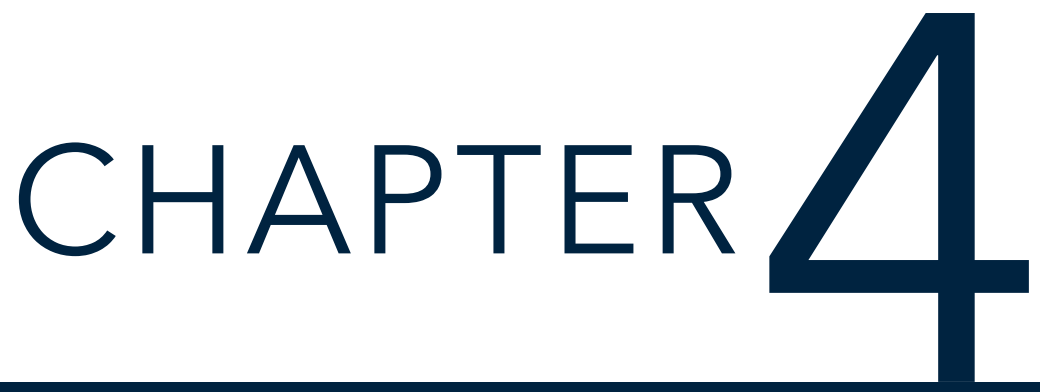

Glucagon-cell Hyperplasia and Neoplasia 


\section{DEFINITION}

Glucagon-cell hyperplasia and neoplasia (GCHN) is the histological phenotype of various rare underlying conditions. By pathogenesis it can be divided in reactive, functional and non-functional GCHN. Reactive GCHN is characterized by germline loss of function mutations in the glucagon receptor gene (GCGR) on chromosome 17q25.3, inherited in an autosomal recessive fashion (Mahvash syndrome (1)). Hyperaminoacidemia, the result of absent glucagon signalling specifically in the liver, causes pancreatic alpha cell hyperplasia and/or neoplasia, which in turn results in hyperglucagonemia without glucagonoma syndrome due to the defective or absent GCGR (2). Because of this, glucagon producing pancreatic neuroendocrine tumors (PanNETs) are per definition non-functional in reactive GCHN. Functional and non-functional GCHN lack GCGR mutations and several other genes in the glucagon signalling pathway have been suggested as pathogenic candidates. Functional GCHN presents with hyperglucagonemia and glucagonoma syndrome, while nonfunctional GCHN lacks both. 


\section{CLINICAL FEATURES}

\section{Incidence}

About half of reported cases of GCHN have been classified as reactive GCHN. While the incidence is unknown, the prevalence of reactive GCHN has been estimated to be about one in four million (3).

\section{Age and sex}

Patients with GCHN present around middle age with gross PanNETs (range 25-74 years). Recently a young girl (7 years) was diagnosed with reactive GCHN after a period of persistent hyperaminoacidemia. Both sexes are equally affected.

\section{Site}

So far, only endocrine pancreatic tissue has been reported to be affected in GCHN. As islets transplanted into the renal capsule of a murine GCHN model also are affected, heterotopic pancreas or metaplasia might also be.

\section{Treatment}

There are no guidelines for treatment. Because of the neoplastic potential treatment and follow-up as recommended for PanNET syndromes like MEN1 has been suggested. PanNETs should be resected following guidelines for non-functional PanNET. Restoring GCGR function would be an attractive treatment but has not been reported yet.

\section{Outcome}

Although cases are very rare, PanNETs develop in most reactive GCHN patients and less commonly in functional/non-functional GCHN. Lymph node and liver metastases are rare but have been reported in reactive and non-reactive GCHN. In the murine model of reactive GCHN, all mice developed microadenomas, most developed macroscopic PanNETs and liver metastases were occasionally seen (3).

\section{PATHOLOGY}

\section{Macroscopy}

Resection is performed for a pancreatic mass, GCHN is usually not suspected clinically. Solid and/or cystic, well-circumscribed tumors can be found on gross examination in an enlarged pancreas. In reactive GCHN, a higher number of (larger) macrotumors can be 

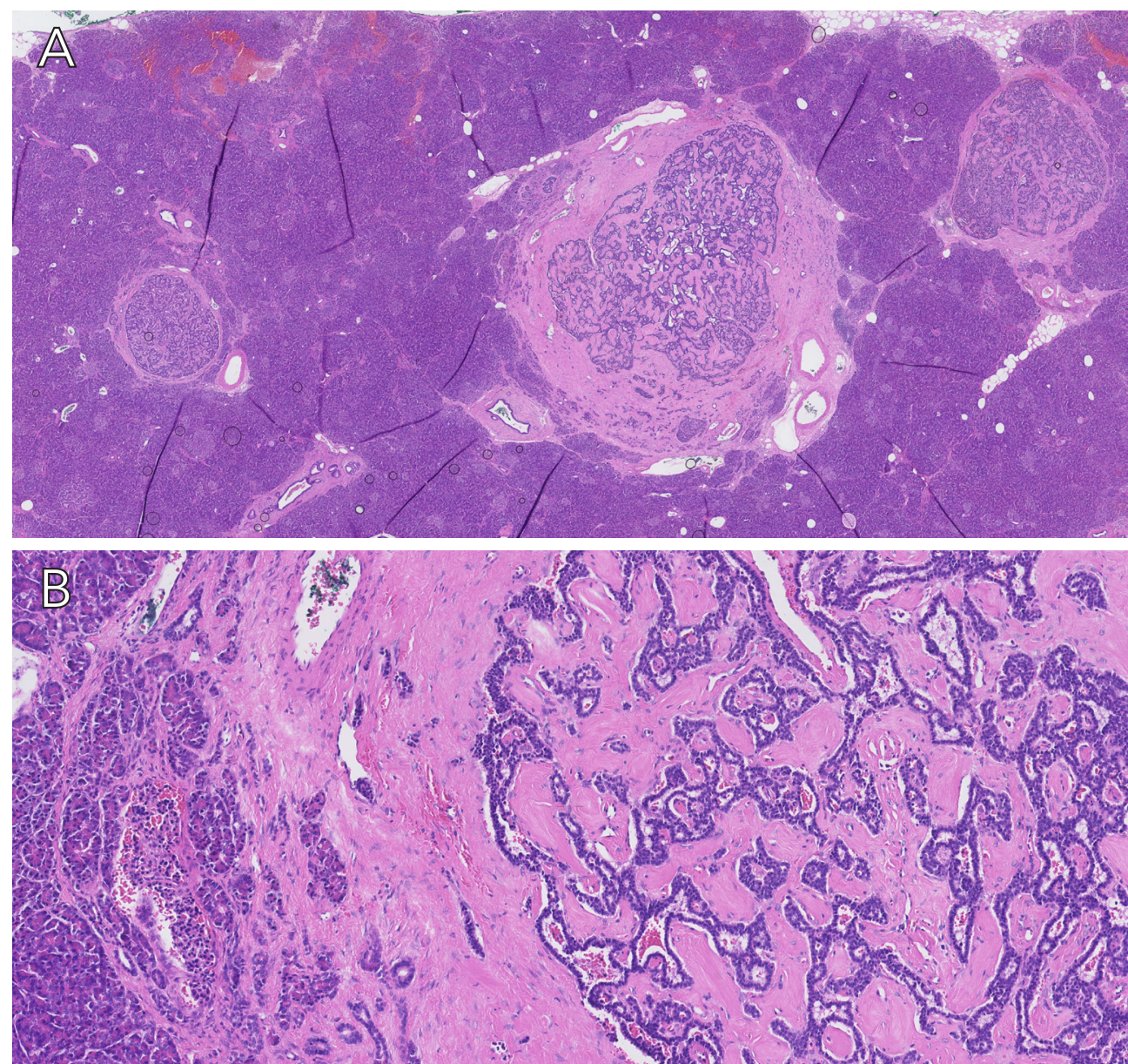

Figure 1. Glucagon-Cell Hyperplasia and Neoplasia. Pancreas with multiple neuroendocrine tumors, microadenomas, and hyperplastic islets, at different magnifications ( $A, 4 x ; B, 20 x$ original magnifications)

found (between 2-12), while fewer macrotumors are seen in non-reactive GCHN (4).

\section{Microscopy}

The pancreas may be diffusely involved and contain several microadenomas $(<5 \mathrm{~mm})$ and PanNETs ( $>5 \mathrm{~mm}$ ), in which a typical neuroendocrine tumor histology can be observed. Tumors can grow in a solid pattern, and calcifications can be found. Numerous (glucagoncell) hyperplastic islets and areas of nesiodioblastosis within ductolinsular structures are scattered through and around the neoplastic lesions. In reactive GCHN, more hyperplastic islets and microadenomas (mean 6.03 to $8.42 / 50 \mathrm{~mm} 2$ ) have been reported compared with non-reactive "wildtype GCGR" GCHN (3-5.16/50 mm2) (4). 

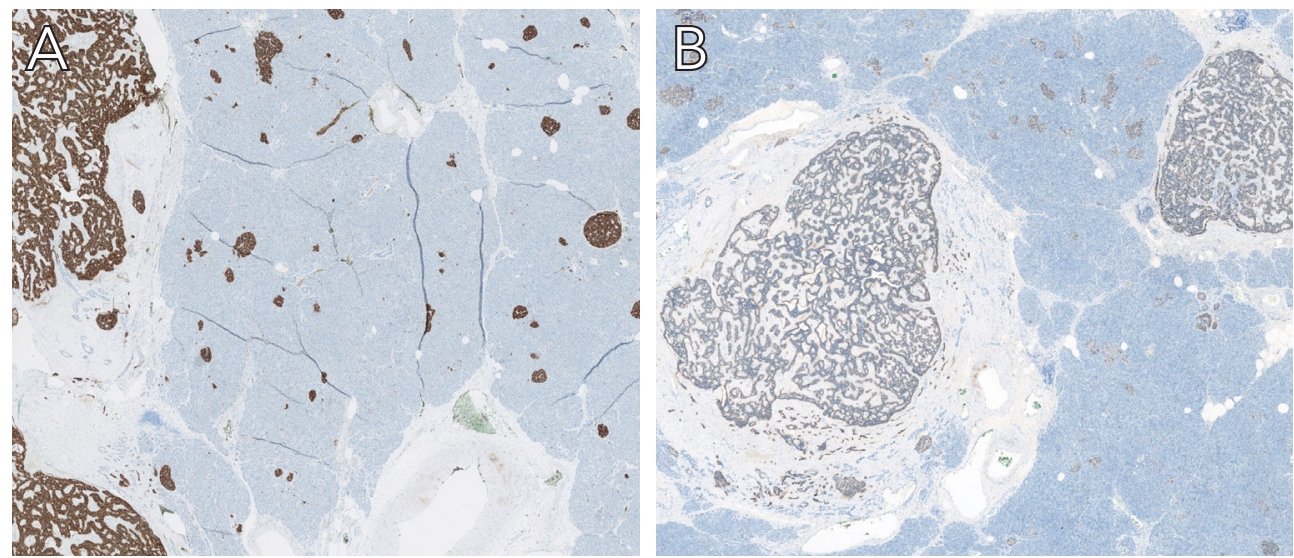

Figure 2. A, glucagon-Cell Hyperplasia and Neoplasia. Intense positivity for synaptophysin (10x original magnification) B, pancreas with multiple glucagon positive pancreatic neuroendocrine tumors, microadenomas and hyperplastic islets. Germline MEN1 or VHL mutations were ruled out in this case. Although GCGR germline mutation status is unknown, asymptomatic hyperglucagonemia is present and the phenotype is consistent with GCHN.

Glucagon immunohistochemistry with haematoxylin counterstaining (10x original magnification).

\section{Immunophenotype}

Tumors are mostly positive for the neuroendocrine markers synaptophysin and chromogranin A. Usually all tumors are positive for glucagon, although glucagon negative tumors have been reported. Pancreatic polypeptide is occasionally positive, all are negative for gastrin and insulin. All hyperplastic islets show predominant or exclusive glucagon expression, while some smaller islets keep normal peptide hormone expression. Ki-67 labeling index is usually less than $2 \%$ for all micro and macro tumors, although $5 \%$ has been reported in larger tumors.

\section{Molecular features}

Germline MEN1 and VHL mutations should be excluded for the diagnosis GCHN. In rare cases somatic mutations and allelic loss of MEN1 have been reported in reactive GCHN microadenomas. It is expected that secondary driving mutations contribute to neoplastic progression.

\section{Differential diagnosis}

MEN1 and VHLsyndrome are two germline genetic conditions with multiple neuroendocrine proliferations throughout the pancreas, and usually have additional clinical manifestations. Pancreatic disease in VHL syndrome is characterized by pancreatic serous cysts, mostly glucagon negative microadenomas and PanNETs, and no glucagon cell hyperplasia (5). In 
MEN1 syndrome, more than half of microadenomas and PanNETs can be glucagon positive and glucagon cell hyperplasia has been reported, but, in contrast to GCHN, other peptide hormone expression can also be observed in micro and macrotumors. In MEN1 as well as VHL syndrome, the number of hyperplastic islets and microadenomas is less than in GCHN (6).

\section{REFERENCES}

1. Yu R, Nissen NN, Dhall D, Heaney AP. Nesidioblastosis and hyperplasia of alpha cells, microglucagonoma, and nonfunctioning islet cell tumor of the pancreas: review of the literature. Pancreas. 2008;36(4):428-31.

2. Solloway MJ, Madjidi A, Gu C, Eastham-Anderson J, Clarke HJ, Kljavin N, et al. Glucagon Couples Hepatic Amino Acid Catabolism to mTOR-Dependent Regulation of alpha-Cell Mass. Cell Rep. 2015;12(3):495-510.

3. Lucas M, Yu V, Yu R. Mahvash disease: pancreatic neuroendocrine tumor syndrome caused by inactivating glucagon receptor mutation. J Mol Genet Med. 2013;7(84):17470862.1000084 .
4.

Sipos B, Sperveslage J, Anlauf M, Hoffmeister M, Henopp T, Buch S, et al. Glucagon cell hyperplasia and neoplasia with and without glucagon receptor mutations. J Clin Endocrino Metab. 2015;100(5):E783-8.

5. Lubensky IA, Pack S, Ault D, Vortmeyer AO, Libutti SK, Choyke PL, et al. Multiple neuroendocrine tumors of the pancreas in von Hippel-Lindau disease patients: histopathological and molecular genetic analysis. Am J Pathol. 1998;153(1):223-31. 6. Anlauf M, Schlenger R, Perren A, Bauersfeld J, Koch $\mathrm{CA}$, Dralle $\mathrm{H}$, et al. Microadenomatosis of the endocrine pancreas in patients with and without the multiple endocrine neoplasia type 1 syndrome. Am J Surg Pathol. 2006;30(5):560-74. 


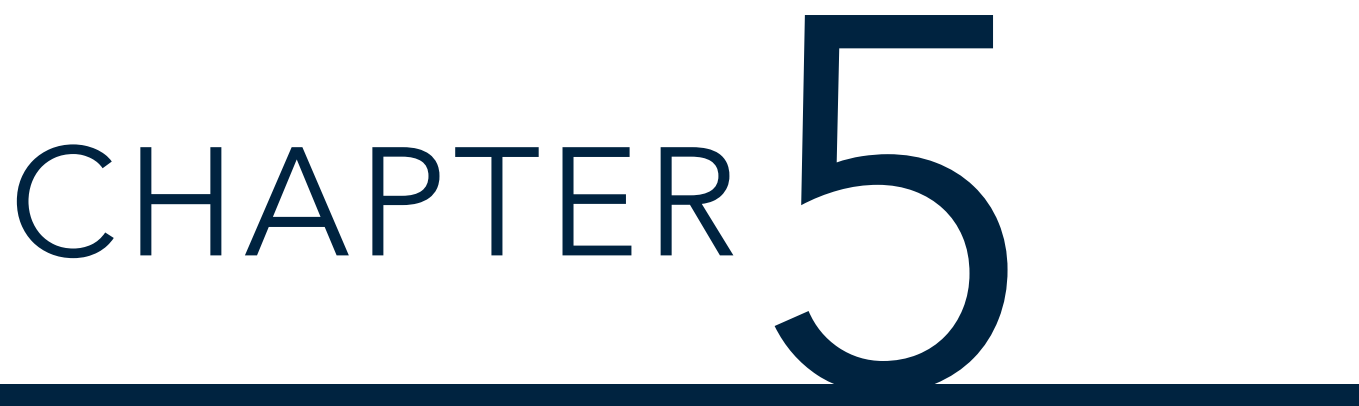

A Parathyroid-Gut Axis: Hypercalcemia and the Pathogenesis of Gastrinoma in Multiple Endocrine Neoplasia 1 


\section{ABSTRACT}

Patients with multiple endocrine neoplasia 1 (MEN1) syndrome have a germline mutation in the MEN1 gene. Loss of the wildtype allele can initiate endocrine tumorigenesis. Microscopic and macroscopic pituitary, parathyroid, and pancreatic tumors (referred to as the 3 P's) show loss of the wildtype MEN1 allele up to $100 \%$. In contrast, the duodenal gastrinoma pathogenesis in MEN1 syndrome follows a hyperplasia-to-neoplasia sequence. Gastrinomas have loss of heterozygosity of the MEN1 locus in $<50 \%$, and invariably coincide with linear, diffuse, or micronodular gastrin-cell hyperplasia. The factor initiating the gastrin-cell hyperplasia-to-neoplasia sequence is unknown. In this perspective, we argue that hypercalcemia may promote the gastrin-cell hyperplasia-to-neoplasia sequence through the calcium sensing receptor. Hypercalcemia is present in almost all patients with MEN1 syndrome due to parathyroid adenomas. We propose a parathyroid-gut axis, which could well explain why patients with MEN1 syndrome are regularly cured of duodenal gastrinoma after parathyroid surgery, and might cause MEN1 syndrome phenocopies in MEN1mutation negative individuals with parathyroid adenomas. This perspective on the pathogenesis of the gastrin-cell hyperplasia and neoplasia sequence sheds new light on tumorigenic mechanisms in neuroendocrine tumors and might open up novel areas of gastrinoma research. It may also shift focus in the treatment of MEN1 syndrome related gastrinoma to biochemical prevention. 


\section{BACKGROUND}

The tissue-specific variation of cancer risk can largely be explained by the normal proliferative activity of cells. Every time a cell divides, there is a small risk of acquiring somatic DNA mutations, which might in turn initiate tumorigenesis. The difference in cancer prevalence arising from colorectal mucosa - with very frequent cell divisions - and endocrine cells of pancreatic islets of Langerhans - with rare cell divisions - is considerable (1). However, in certain instances, the odds are stacked against the endocrine cells. Patients with MEN1 syndrome have a germline inactivating Multiple Endocrine Neoplasia 1 gene (MEN1) mutation. Menin, the MEN1 protein product, acts as a tumor suppressor in endocrine cells (2), and inactivation of both alleles can initiate tumorigenesis. Following Knudson's twohit hypothesis, loss of heterozygosity $(\mathrm{LOH})$ at the MEN1 locus on chromosome 11q13 or somatic mutation of the MEN1 wild-type allele is sufficient to initiate tumorigenesis, drastically increasing the risk of endocrine cancer, also in the pancreatic islets. Of note, the tumor suppressive effect of Menin is well-established in certain MEN1-associated endocrine organs, such as the parathyroid glands and the endocrine pancreas, but its role as tumor suppressor is more controversial in other cell-types. For example, Menin may have a dual oncogene activating/tumor suppressive role in breast cancer and prostate cancer (3-6).

Indeed, parathyroid adenomas and pituitary tumors develop in more than $90 \%$ and $40 \%$ of patients with MEN1 syndrome, respectively (7). Duodenopancreatic neuroendocrine tumors (NETs) are seen in virtually all patients with MEN1 syndrome (8). Endocrine tumors arising in patients with MEN1 syndrome often produce hormones causing clinical symptoms. Most patients have hypercalcemia due to excessive parathyroid hormone (PTH) secretion. Some patients may develop hypoglycemic events due to pancreatic insulinoma, or Zollinger Ellison Syndrome [ZES] i.e., increased gastric acid secretion due to duodenal gastrinomas.

In accordance with the Knudson theory, patients with MEN1 syndrome harbor loss of the wild-type MEN1 allele by LOH in 100\% of parathyroid adenomas (9), 100\% of pituitary tumors $(10,11), 83-90 \%$ of pancreatic NETs and 100\% of pancreatic microadenomas (neuroendocrine proliferation $<5 \mathrm{~mm})(9,12-14)$, while rare cases without $\mathrm{LOH}$ have somatic loss of function mutations of the wildtype allele (10). Remarkably, duodenal gastrinomas in MEN1 syndrome show a very different picture, with LOH of the MEN1 locus in only 21-46\% of tumors $(9,12,15)$. It was also noted that duodenal gastrinomas in MEN1 syndrome were invariably surrounded by areas of linear, nodular and diffuse enteroendocrine gastrin-cell 
(G-cell) hyperplasia, none of which had loss of the MEN1 locus at chromosome 11q13 (15). This is intriguing, because it strongly suggests that loss of the wild-type MEN1 allele is not the initiating driver of the G-cell proliferations and hyperplasia, and not a prerequisite for the multifocal neuroendocrine tumors in the duodenum. As a result, a duodenal G-cell hyperplasia to neoplasia sequence was proposed (16). However, if MEN1 does not stack the odds against $\mathrm{G}$-cells, what factor does $(17,18)$ ?

\section{HYPOTHESIS}

The nature of endocrine cells is to secrete enough hormones to meet demand. Overstimulation therefore commonly drives endocrine cell proliferation. As proliferation/ hyperplasia is associated with an increased risk of mutations, this results in increased tumor formation(1). Indeed, this is the case in the rare glucagon cell hyperplasia and neoplasia $(\mathrm{GCHN})$ syndrome $(19,20)$, affecting the pancreatic islets. In GCHN, a defective glucagon receptor results in reduced ureagenesis and gluconeogenesis related amino acid uptake in the liver. The resulting hyperaminoacidemia - the stimulating factor - promotes alphacell proliferation and hyperplasia, a feedback loop aiming to increase glucagon levels and restore the liver-alpha-cell axis (21). These proliferations increase the chance of acquiring mutations, amongst others in MEN1, eventually leading to development of multiple pancreatic alpha-cell tumors (20). Could a similar mechanism play a role in G-cell hyperplasia to neoplasia, especially in the setting of increased vulnerability due to germline heterozygous MEN1 mutations?

G-cells are present in the gastric antrum and duodenum, and are stimulated to release gastrin by luminal peptones or amino acids, parasympathetic vagal nerve reaction to stomach distention, and - notably - extracellular increase of calcium levels $(22,23)$. Hypercalcemia, commonly seen in MEN1 syndrome due to parathyroid adenomas, might therefore play a key role in initiating G-cell hyperplasia.

G-cells have a calcium-sensing receptor (CaSR) (22), that is also expressed in gastrinomas causing ZES (24). The ability of G-cells to react to calcium has long been exploited to support the diagnosis of ZES after intravenous calcium gluconate injection. Recently, it was shown in a murine model that CaSR in fact functions as a regulator of G-cell growth, and CaSR null mice have low numbers of G-cells (25). Moreover, in human gastric biopsies in the setting of absorptive hypercalciuria, characterized by increased CaSR sensitivity, G-cell 


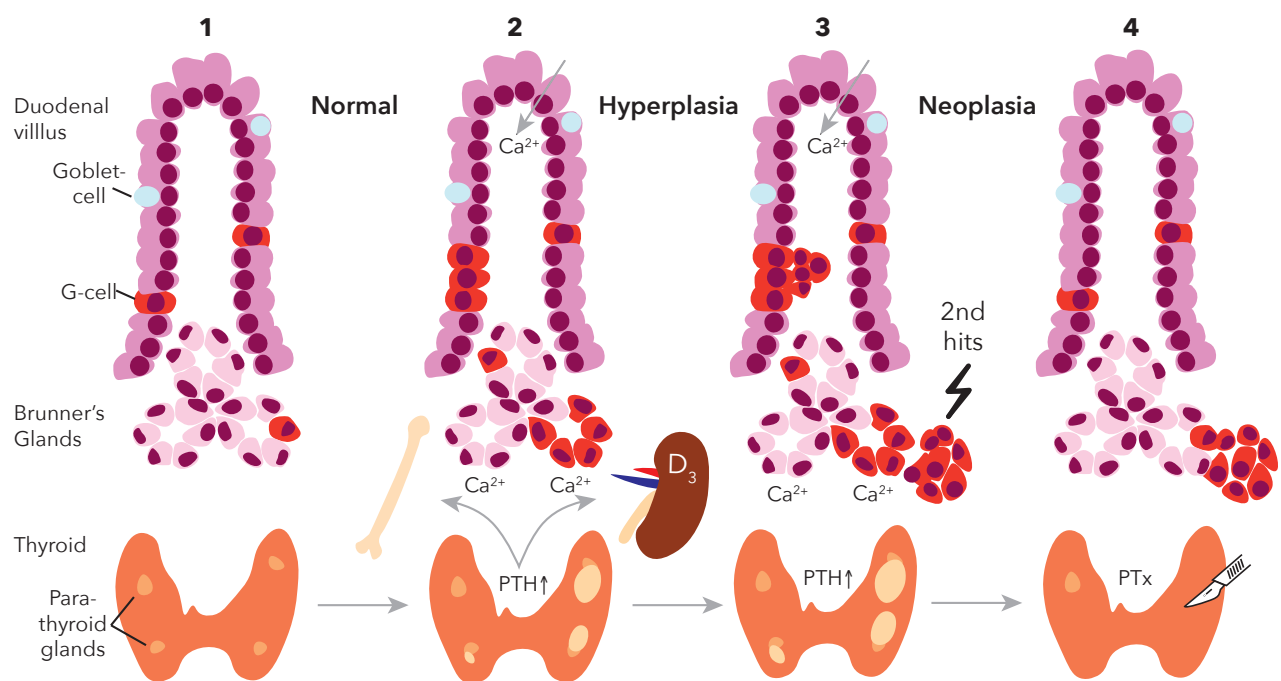

Figure 1. Hypothetical gastrinoma hyperplasia to neoplasia sequence in multiple endocrine neoplasia 1 (MEN1) syndrome. 1) Normal physiological condition of parathyroid glands and duodenal mucosa. 2) The development of parathyroid adenomas increases parathyroid hormone levels, which in turn results in hypercalcemia through its effects on the bones, kidneys and intestine. 3) Hypercalcemia may stimulate G-cell proliferation, which increases the risk of second hit inactivation of MEN1. 4) Progression of hyperplasia to neoplasia, by biallelic MEN1 inactivation, may cause independence over the initial stimulus, which may be reduced after parathyroid surgery.

hyperplasia was seen in all patients. In addition, nodular gastric ECL-cell hyperplasia and fundic gland polyps were observed in some cases, as often seen in ZES (26).

Almost all MEN1 syndrome patients develop parathyroid adenomas and experience hypercalcemia during their life. Similarly, duodenal G-cell hyperplasia is found in all patients with MEN1 (17). Furthermore, although the symptoms of ZES sometimes precede those of hyperparathyroidism, almost all patients with ZES have hyperparathyroidism (27). Studies have shown that following successful parathyroidectomy in MEN1 syndrome patients with ZES, $20 \%$ of patients were biochemically cured of ZES, without removing any duodenal or pancreatic NET (28). In line with this observation, chronic sporadic primary hyperparathyroidism may mimic MEN1 syndrome due to development of ZES or duodenal gastrinomas, thereby fulfilling the clinical criteria for MEN1 syndrome: gastrinomas were found to be common in MEN1 mutation-negative MEN1 syndrome probands, of which 90\% had primary hyperparathyroidism (29). In contrast with MEN1 mutation-positive probands, recurrent primary hyperparathyroidism was seen less often ( $9 \%$ versus $56 \%$ ). 
As calcium is well known to provoke gastrin secretion, parathyroid surgery can cure ZES, and CaSR plays a role in regulating the number of G-cells present in the mucosa, it is plausible that parathyroid disease plays a causal role in G-cell hyperplasia. Following the paradigm that the cancer risk of a particular cell type can in part be explained by the proliferative activity, this would imply that hyperparathyroidism may also increase risk of G-cell neoplasia (gastrinoma). In the setting of MEN1 syndrome, the heterozygous germline MEN1 defect makes G-cells even more prone to neoplastic progression, independent of their initial hormonal stimulus (Figure 1). However, it is clear that a proportion of duodenal gastrinomas will remain (functionally) dependent of their growth stimulus, as evidenced by the cured ZES patients after parathyroidectomy (28).

\section{CLINICAL CONSEQUENCES AND RESEARCH PROPOSALS}

Currently, indications for parathyroid surgery in MEN1 syndrome include hypercalciuria, nephrolithiasis, symptomatic hypercalcemia and reduced bone density. Surgery on young MEN1 syndrome patients with asymptomatic hyperparathyroidism is controversial in view of the risk of recurrence and more complicated re-surgery. However, in the light of the possible G-cell hyperplasia-neoplasia sequence in the duodenum, early presymptomatic control of biochemical parameters would be an interesting strategy to reduce the chance of developing malignant gastrinoma. The calcimimetic agent cinacalcet has shown to reduce the hypercalcemia by increasing CaSR sensitivity for extracellular calcium (30). CaSR is also expressed on the parathyroid chief cells secreting PTH, and under normal circumstances, calcium negatively regulates PTH secretion. By allosteric binding, cinacalcet increases calcium sensitivity and reduces PTH secretion, in turn decreasing calcium levels. However, it remains to be studied if increasing CaSR sensitivity might also stimulate G-cell proliferation, comparable to absorptive hypercalciuria (26), and eventually increase the risk of G-cell tumors, especially in the setting of a germline MEN1 mutation. Of note, treatment with cinacalcet has indeed been demonstrated to increase gastrin concentrations and gastric acid secretion in healthy individuals (31).

Unfortunately, no in vivo gastrinoma tumor model is available. The lack of gastrinomas in many murine MEN1 syndrome models has been puzzling, but importantly, MEN1 mice regularly lacked parathyroid adenomas or hypercalcemia (32). Mice with a gastrointestinal epithelium-specific deletion of Men1 might be especially suited to study the effect of cinacalcet or primary hyperparathyroidism on duodenal gastrinoma development (33). Studying human G-cell hyperplasia on biopsies pre- and post-parathyroid surgery 
would be very challenging, especially as it is yet unclear what the effect of proton pump inhibitors, calcium supplementation or cinacalcet might have on G-cell proliferation. Nevertheless, supported by extensive literature, we hypothesize that it is plausible that parathyroid adenomas enhance duodenal G-cell and gastrinoma activity. By promoting G-cell proliferation, hyperparathyroidism-induced hypercalcemia may be the missing link in the hyperplasia to neoplasia sequence of gastrinomas in MEN1 syndrome. The proposed parathyroid-gut axis might present novel gastrinoma treatment targets and opportunities. Early biochemical cure of parathyroid disease may decrease disease burden related to G-cell proliferations in MEN1 syndrome patients.

\section{CONFLICT OF INTEREST:}

the authors declare no potential conflicts of interest

\section{REFERENCES}

1. Tomasetti C, Vogelstein B. Cancer etiology. Variationin cancer risk among tissues can be explained by the number of stem cell divisions. Science. 2015;347(6217):78-81.

2. Dreijerink KMA, Timmers HTM, Brown M. Twenty years of menin: emerging opportunities for restoration of transcriptional regulation in MEN1. Endocr Relat Cancer. 2017;24(10):T135-T45.

3. Caslini C, Yang Z, El-Osta M, Milne TA, Slany RK, Hess $\mathrm{JL}$. Interaction of MLL amino terminal sequences with menin is required for transformation. Cancer Res. 2007;67(15):7275-83. 4. Imachi H, Murao K, Dobashi H, Bhuyan MM, Cao $X$, Kontani K, et al. Menin, a product of the MENI gene, binds to estrogen receptor to enhance its activity in breast cancer cells: possibility of a novel predictive factor for tamoxifen resistance. Breast Cancer Res Treat. 2010;122(2):395-407.

5. Dreijerink KMA, Groner AC, Vos ESM, Font-Tello A, Gu L, Chi D, et al. Enhancer-Mediated Oncogenic Function of the Menin Tumor Suppressor in Breast Cancer. Cell Rep. 2017; 18(10):2359-72.

6. Malik R, Khan AP, Asangani IA, Cieslik M, Prensner JR, Wang $X$, et al. Targeting the MLL complex in castration-resistant prostate cancer. Nat Med. 2015;21(4):344-52.

7. Verges B, Boureille F, Goudet P, Murat A, Beckers A, Sassolas G, et al. Pituitary disease in MEN type 1 (MEN1): data from the France-Belgium MEN1 multicenter study. J Clin Endocrinol Metab. 2002;87(2):457-65.

8. Anlauf M, Schlenger R, Perren A, Bauersfeld J, Koch $\mathrm{CA}$, Dralle $\mathrm{H}$, et al. Microadenomatosis of the endocrine pancreas in patients with and without the multiple endocrine neoplasia type 1 syndrome. Am J Surg Pathol. 2006;30(5):560-74.

9. Lubensky IA, Debelenko LV, Zhuang Z, Emmert-Buck MR, Dong Q, Chandrasekharappa S, et al. Allelic deletions on chromosome $11 \mathrm{q} 13$ in multiple tumors from individual MEN1 patients. Cancer Res. 1996;56(22):5272-8.
10. Pannett AA, Thakker RV. Somatic mutations in MEN type 1 tumors, consistent with the Knudson "two-hit" hypothesis. J Clin Endocrinol Metab. 2001;86(9):4371-4.

11. Weil RJ, Vortmeyer AO, Huang S, Boni R, Lubensky IA, Pack $S$, et al. 11q13 allelic loss in pituitary tumors in patients with multiple endocrine neoplasia syndrome type 1. Clin Cancer Res. 1998;4(7):1673-8.

12. Debelenko LV, Zhuang Z, Emmert-Buck MR, Chandrasekharappa SC, Manickam P, Guru SC, et al. Allelic deletions on chromosome 11q13 in multiple endocrine neoplasia type 1 -associated and sporadic gastrinomas and pancreatic endocrine tumors. Cancer Res. 1997;57(11):2238-43.

13. Hessman $O$, Lindberg D, Einarsson A, Lillhager $P$, Carling T, Grimelius L, et al. Genetic alterations on $3 p, 11 q 13$, and $18 q$ in nonfamilial and MEN 1-associated pancreatic endocrine tumors. Genes Chromosomes Cancer. 1999;26(3):258-64.

14. Perren A, Anlauf M, Henopp T, Rudolph T, Schmitt A, Raffel A, et al. Multiple endocrine neoplasia type 1 (MEN1): loss of one MEN1 allele in tumors and monohormonal endocrine cell clusters but not in islet hyperplasia of the pancreas. J Clin Endocrinol Metab. 2007;92(3):1118-28.

15. Anlauf M, Perren A, Henopp T, Rudolf T, Garbrecht $\mathrm{N}$, Schmitt A, et al. Allelic deletion of the MEN1 gene in duodenal gastrin and somatostatin cell neoplasms and their precursor lesions. Gut. 2007;56(5):637-44.

16. Anlauf M, Perren A, Meyer CL, Schmid S, Saremaslani $\mathrm{P}$, Kruse ML, et al. Precursor lesions in patients with multiple endocrine neoplasia type 1-associated duodenal gastrinomas. Gastroenterology. 2005;128(5):1187-98.

17. Kloppel G, Anlauf M, Perren A, Sipos B. Hyperplasia to neoplasia sequence of duodenal and pancreatic neuroendocrine diseases and pseudohyperplasia of the PP-cells in the pancreas. Endocr Pathol. 2014;25(2):181-5.

18. Pritchard DM. Pathogenesis of gastrinomas 
associated with multiple endocrine neoplasia type 1. Gut. 2007; 56(5):606-7

19. Yu R, Nissen NN, Dhall D, Heaney AP. Nesidioblastosis and hyperplasia of alpha cells, microglucagonoma, and nonfunctioning islet cell tumor of the pancreas: review of the literature. Pancreas. 2008;36(4):428-31.

20. Henopp T, Anlauf M, Schmitt A, Schlenger R, Zalatnai

A, Couvelard A, et al. Glucagon cell adenomatosis: a newly recognized disease of the endocrine pancreas. J Clin Endocrinol Metab. 2009;94(1):213-7.

21. Solloway MJ, Madjidi A, Gu C, Eastham-Anderson J, Clarke HJ, Kljavin N, et al. Glucagon Couples Hepatic Amino Acid Catabolism to mTOR-Dependent Regulation of alpha-Cell Mass. Cell Rep. 2015;12(3):495-510.

22. Ray JM, Squires PE, Curtis SB, Meloche MR, Buchan AM. Expression of the calcium-sensing receptor on human antral gastrin cells in culture. J Clin Invest. 1997;99(10):2328-33.

23. Boron WF, Boulpaep EL. Medical physiology: Elsevier Health Sciences; 2016

24. Goebel SU, Peghini PL, Goldsmith PK, Spiegel AM, Gibril F, Raffeld M, et al. Expression of the calcium-sensing receptor in gastrinomas. J Clin Endocrinol Metab. 2000;85(11):4131-7. 25. Feng J, Petersen CD, Coy DH, Jiang JK, Thomas CJ, Pollak MR, et al. Calcium-sensing receptor is a physiologic multimodal chemosensor regulating gastric G-cell growth and gastrin secretion. Proc Natl Acad Sci U S A. 2010;107(41):17791-6.

26. Declich P, Belloni J, Tavani E, Omazzi B, Bellone S, Bortoli $A$, et al. The morphological and immunohistochemical spectrum of gastric biopsies of patients with absorptive hypercal- ciuria. BMJ Open Gastroenterol. 2014;1(1):e000004.

27. Jensen RT, Cadiot G, Brandi ML, de Herder WW,

Kaltsas G, Komminoth P, et al. ENETS Consensus Guidelines for the management of patients with digestive neuroendocrine neoplasms: functional pancreatic endocrine tumor syndromes. Neuroendocrinology. 2012;95(2):98-119.

28. Norton JA, Venzon DJ, Berna MJ, Alexander HR,

Fraker DL, Libutti SK, et al. Prospective study of surgery for primary

hyperparathyroidism (HPT) in multiple endocrine neoplasia-type

1 and Zollinger-Ellison syndrome: long-term outcome of a more

virulent form of HPT. Ann Surg. 2008;247(3):501-10.

29. Kovesdi A, Toth M, Butz H, Szucs N, Sarman B, Pusztai

$P$, et al. True MEN1 or phenocopy? Evidence for geno-phenotypic correlations in MEN1 syndrome. Endocrine. 2019;65(2):451-9.

30. Giusti F, Cianferotti L, Gronchi G, Cioppi F, Masi

L, Faggiano A, et al. Cinacalcet therapy in patients affected by primary hyperparathyroidism associated to Multiple Endocrine Neoplasia Syndrome type 1 (MEN1). Endocrine. 2016;52(3):495506.

31. Ceglia L, Harris SS, Rasmussen HM, Dawson-Hughes B. Activation of the calcium sensing receptor stimulates gastrin and gastric acid secretion in healthy participants. Osteoporos Int. 2009;20(1):71-8.

32. Mohr H, Pellegata NS. Animal models of MEN1.

Endocr Relat Cancer. 2017;24(10):T161-T77.

33. Veniaminova NA, Hayes MM, Varney JM, Merchant JL. Conditional deletion of menin results in antral $\mathrm{G}$ cell hyperplasia and hypergastrinemia. Am J Physiol Gastrointest Liver Physiol. 2012;303(6):G752-64 


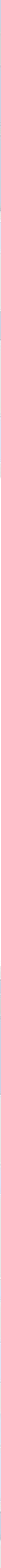




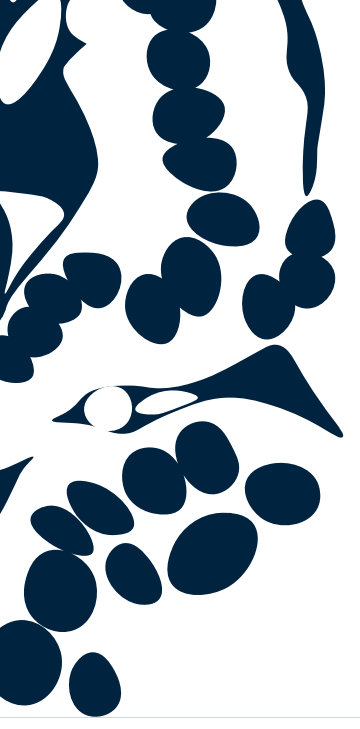

EPIGENETIC DIFFERENTIATION REFINES NEUROENDOCRINE TUMOR CLASSIFICATION
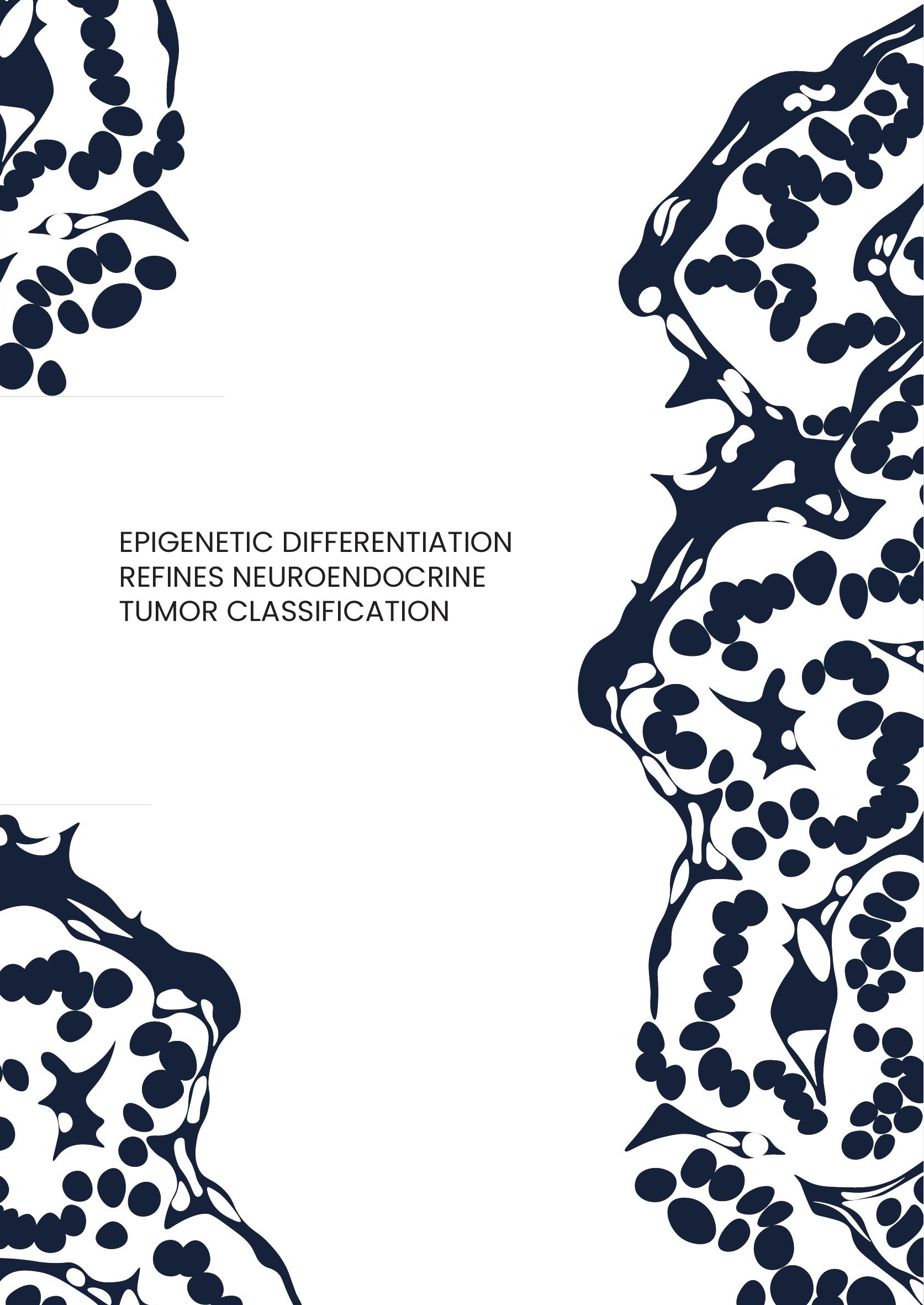


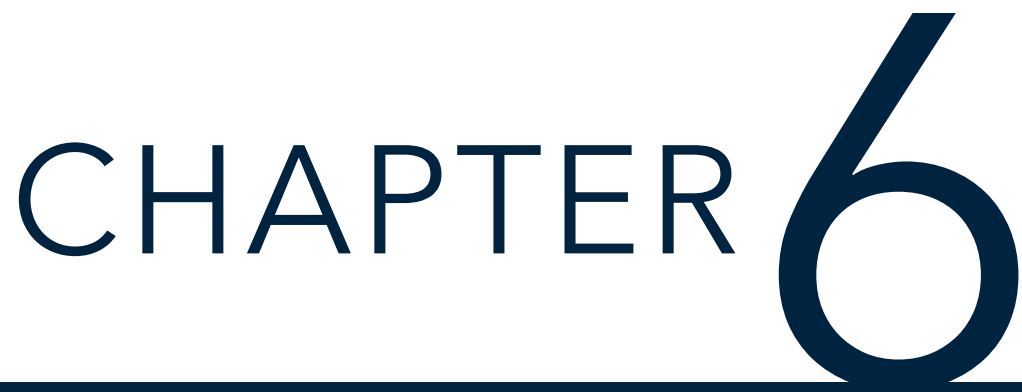

Assessment of ARX expression, a novel biomarker for metastatic risk in pancreatic neuroendocrine tumors, in endoscopic ultrasound fine needle aspiration 


\section{ABSTRACT}

The transcription factors ARX and PDX1, and alternative lengthening of telomeres (ALT) were recently described as prognostic markers for resected non-functional pancreatic neuroendocrine tumors (PanNETs). ALT positive tumors with ARX expression relapse most often. Currently, tumor size is the only pre-operative marker used to decide whether or not to operate, thus additional pre-operative prognostic markers are needed. Therefore, it is critical to assess the performance of these biomarkers on pre-operative cytologic specimens.

Endoscopic fine needle aspiration cellblock material and corresponding surgical specimens of 13 patients with PanNETs were assessed for histology, immunohistochemical staining of ARX, PDX1, Synaptophysin, and Ki67, telomerespecific fluorescence in situ hybridization to detect ALT, and then associated with clinicopathological features. Scoring for ARX and PDX1 was performed blinded by two independent observers.

Of the 13 surgical specimens, 8 were ARX+/PDX1-, 2 ARX-/PDX1+, and 3 ARX+/ PDX1+. Concordance between cytologic and surgical specimens for ARX protein expression was 100\%, whereas concordance for PDX1, ALT, and WHO tumor grade was $85 \%, 91 \%$ and $73 \%$, respectively. There was a perfect inter-observer agreement in ARX and PDX1 scoring.

ARX can reliably be determined in cytologic specimens and has low inter-observer variability. For cytology, false positive PDX1 expression was observed, possibly due to contamination or sampling, while ALT had a false negative case due to incomplete sampling. As previously observed, tumor grade is underestimated in cytologic specimens. Thus, ARX and ALT are the most promising markers to predict metastatic behavior in PanNETs, thereby warranting further validation in larger studies. 


\section{INTRODUCTION}

Neuroendocrine tumors of the pancreas (PanNET) are the second most common malignancy of the pancreas. Hepatic metastases are the leading cause of death in these patients and almost half have metastatic liver involvement at first presentation (1). However, for the other half, preventing liver metastases is the primary goal for treatment and followup, in particular for non-functional cases which comprise the largest proportion of PanNETs. Surgical resection of the primary tumor can reduce the risk of metastasis, but is associated with significant morbidity and mortality. Importantly, surgery may be unnecessary in some cases, as a subgroup of PanNETs is indolent and does not give metastases. The choice for surgery - a trade-off between the associated risks of surgery, post-surgical morbidity and the potential benefit of preventing metastasis - is difficult, as reliable pre-operative prediction of indolent or aggressive biology is lacking. Most established prognostic factors for development of metastasis, such as microscopic invasiveness, lymph node involvement, perineural invasion, and tumor grade can only reliably be determined after surgery. The only marker with prognostic value that can easily be pre-operatively assessed is tumor size, based on CT- or MR-imaging or endoscopic ultrasound, which is used as the key criterion to decide on surgery in non-functional PanNETs in European and American guidelines $(2,3)$. Thus, additional markers for pre-operative risk stratification are urgently needed.

Larger tumor size is an independent predictor of disease relapse after surgery, nevertheless multiple retrospective studies show that most patients with large tumors do not have recurrent disease or liver metastasis after resection, and are histologically "benign" grade 1 tumors without invasion or involved lymph nodes. It is likely that at least a subgroup of these patients could benefit from conservative therapy, as their tumors might actually be indolent, despite the large tumor size. Additional markers to identify this subgroup are necessary to reduce potential over-treatment of these patients. Conversely, pre-operative markers to identify the aggressive PanNETs of smaller size could be useful to identify patients that require earlier surgery instead of "wait and scan".

Several markers have the potential to identify high and low risk subgroups. PanNET tumorigenesis is commonly driven by mutations in the tumor suppressor genes MEN1, ATRX or DAXX, of which the latter two are associated with the alternative lengthening of telomeres phenotype (ALT) and correlate with worse prognosis and liver metastases (49). Protein loss, detected by immunohistochemistry (IHC), can be used as a surrogate for 
somatic inactivating mutations in $\operatorname{ATRX}$ and $\operatorname{DAXX}(9,10)$. Recently, promising results in determining these markers in pre-operative cytologic specimens have been published (11). Nevertheless ATRX/DAXX IHC poses some difficulties, such as the need for positive internal controls, intratumoral heterogeneity, and presence of mutations (e.g. missense) that alter function, but not protein expression or localization. Although assessment of ALT is more reliable, nearly $50 \%$ of metastatic PanNETs do not have ATRX/DAXX alterations or ALT, thereby emphasizing the need for additional predictive biomarkers (5).

Recent studies demonstrate that most PanNETs fall into two major subtypes, based on $\mathrm{H} 3 \mathrm{~K} 27 \mathrm{ac}$ and H3K4me2 signatures, which resemble either normal islet alpha or beta cells. As a surrogate marker of these subtypes, endocrine transcription factors ARX (alpha cell) and PDX1 (beta cell) can be used, and more than half of functional and non-functional PanNETs showed mutually exclusive expression of these transcription factors. Intriguingly, relapse was almost exclusively associated ARX positive, PDX1 negative or double negative group. PDX1 or double positive expression on the other hand identified a more indolent group, in which only few tumors relapsed (12). Similarly, Chan et al showed, using RNA sequencing and whole genome methylation, that the ARX positive, PDX1 negative subgroup was often associated with somatic mutations in ATRX, DAXX or MEN1 and a worse prognosis (13).

Thus, ARX and PDX1, in combination with ALT, may be prognostic markers to identify low and high-risk subgroups pre-operatively on cytology, as staining of these proteins seems to identify these alpha and beta cell-like subgroups robustly. Although, a prerequisite to be able to consider these markers for routine clinical use is to determine if cytologic material can be reliably used to detect tumor subtype. In addition, inter-observer agreement between pathologists must be high and methodology for sampling by the gastroenterologist must be established and reproducible. This study aims to answer these questions and provide the framework for further optimization of ARX and PDX1 staining in combination with ALT as preoperative markers, thereby justifying independent validation of these markers in large, prospective trials.

\section{METHODS}

\section{Patient materials}

This study was approved by the UMC Utrecht Biobank Research Ethics Committee. The pathology archives were searched for cytology paraffin blocks and corresponding surgical specimens with the diagnosis neuroendocrine tumor/atypical cells of the pancreas. If paraffin blocks were available, the presence of tumor material was confirmed by a 
hematoxylin and eosin stained slide. Data was collected from the pathology report (age, gender, macroscopic size, grade, lymph nodes, margins, type of paraffin block) and patient files (hormone production, genetic syndromes, endoscopic ultrasound aspiration or biopsy, tumor size, follow-up, development of liver metastases).

\section{Immunohistochemistry and Fluorescence in Situ Hybridization}

Consecutive $4 \mu \mathrm{m}$ sections of formalin-fixed paraffin embedded tissue per case were cleared for 10 minutes at $60 \mathrm{C}$ and deparaffinized in xylene. Endogenous peroxidase was blocked by immersion in 0,6\% H2O2 (Merck 7210, Kenilworth, NJ) in methanol for 15 minutes. Antigen retrieval was performed by boiling slides in a $10 \mathrm{mM}$ citrate solution ( $\mathrm{pH} 6$ ) for 20 minutes or a 10/1 mM Tris/EDTA solution ( $\mathrm{pH}$ 9) in the case of synaptophysin. Nonspecific binding was reduced by blocking with Protein Block Serum Free (Dako, Santa Clara, CA, X0909,). ARX (Millipore Burlington, MA, MABN102, clone 11F6.2, 1:2000), PDX1 (Abcam, Cambridge, United Kingdom, AB134150, clone EPR 3358(2), 1:2000), Synaptophysin (Novocastra, Amsterdam, The Netherlands, NCL-L-SYNAP-299, clone 27G12, 1:100) and Ki67 (Thermo Fisher, MA, USA, SP6, Lot. 9106S1805D, 1:100) antibodies were diluted in Normal Antibody Diluent (Immunologic, Duiven, The Netherlands) and incubated for 60 minutes at room temperature. After post antibody blocking (Immunologic) for 15 minutes, the secondary antibody Poly-HRP-goat anti Mouse/Rabbit lgG (Immunologic, Cat. no.VWRKDPVB110HRP) was incubated for 30 minutes. Peroxidase activity was detected by DAB (Sigma, St. Louis, MO D5637) or Bright-DAB for Ki67 slides (cat. no. VWRKBS04-110, Immunologic) as chromogen for 8 minutes. Slides were shortly counterstained with hematoxylin and coverslips were mounted with Pertex (Histolab, Askim, Sweden).

The telomere-specific fluorescence in situ hybridization (FISH) was performed as previously described (14). Briefly, cleared and deparaffinized sections were boiled in $10 \mathrm{mM}$ citrate solution ( $\mathrm{pH}$ 6) for 20 minutes. Slides were washed, dried, and the telomere probe (TelC Cy3, F1002 Lot no. 180723PL-01, 100nM, Panagene, Yuseong-gu, Republic of Korea) and the hybridization control centromere probe (Cent-FITC F3013 Lot No. 172865, 100nM, Panagene) diluted in hybridization mix (50\% deionized Formamide, 50\% SCC 4x, 5\% Dextran sulfate, Tween-20 0.5\%) were added. Slides were covered and after 5 minutes denaturing at $84^{\circ} \mathrm{C}$, slides were quickly cooled and hybridized overnight at $37 \mathrm{C}$. The next day, slides were washed in two cycles of 3 times PBS and 1 time PNA wash buffer (70\% Formamide, 30\% dH2O, 10mM Tris). Slides were then counterstained with DAPI in PBS 2ug/ml (Sigma-Aldrich, D9542). Slides were washed with dH20, dried and mounted 
with Vectashield (H-1000, Vector laboratories, Amsterdam, The Netherlands) and cooled before imaging. Imaging was done with a DM5500 B epifluorescence microscope (Leica Microsystems, Rijswijk, The Netherlands). Photos were made with Leica application Suite X with a $Z$ stack of 14 steps.

All scoring was performed in a blinded fashion for clinical or case specific information. Areas with clumped synaptophysin positive cells in the cytology were annotated for scoring of Ki67, ARX, and PDX1 expression and for assessment of telomere FISH. ARX and PDX1 $\mathrm{IHC}$ scoring was performed by two independent researchers $(\mathrm{WMH}$, a medical doctor with more than 4 years of experience in PanNET research, and LAAB, a gastrointestinal pathologist with 10 years of experience in NET diagnostics and research) blinded for each other's results. Both researchers had previous experience with scoring ARX and PDX1 on whole tumor and tissue microarray specimens. Disagreements (if present) were resolved by consensus. Negative protein expression in tumor tissue was defined as weak nuclear staining of ARX or PDX 1 in $<50 \%$ of cells or strong nuclear staining in $<10 \%$ of cells. Positive ARX or PDX1 expression was called if weak- nuclear staining was present in $>50 \%$ of cells or strong nuclear staining $>10 \%$ of cells as previously described.

The Ki67 labeling index was counted in at least 2000 cells in for the surgical specimens by digital image analysis with Sectra (PACS, Sectra AB, Linköping, Sweden) in the areas with highest labeling as previously described.(15) For the cytologic specimens, all cells in the synaptophysin labeled areas were digitally counted. All digital counts were visually confirmed. Grading was performed following 2017 WHO classification based on Ki67 proliferation index (16). If the pathology report mentioned higher tumor grade than the Ki67 count for the surgical specimens, the highest grade was used for the tumor characteristics and dichotomous comparisons with cytology, as higher labeling areas could have been missed in the tested slides. For the continuous comparison between surgical specimens and cytology, the digitally measured Ki67 labeling index was used.

ALT positivity was defined as ultra-bright, intranuclear telomere FISH signals, more than 10x the signal intensity of combined telomeres of normal stromal/endothelial cells, if present in $>1 \%$ of cells determined by visual assessment at $20 \times(\mathrm{WMH})$ (9). For the surgical specimens, 100x magnification greyscale images were obtained of areas with suspected ultrabright foci surrounded by stromal cells, and telomere signals were quantified and confirmed using Telometer (a free custom software ImageJ plug-in, downloaded from demarzolab. 
Table 1. Patient characteristics

\begin{tabular}{|c|c|}
\hline & All cases \\
\hline Included cases: H\&E cytology representative, n (\%) & $20(100)$ \\
\hline Formalin-fixed paraffin-embedded & 2 \\
\hline Formalin-fixed agar-gelatin paraffin-embedded & 12 \\
\hline CytoLyt-fixed and formalin -fixed paraffin-embedded & 5 \\
\hline CytoLyt-fixed Cellient & 1 \\
\hline Male gender, n (\%) & $13(65)$ \\
\hline Age \pm SD (yr.) & $52.9(14.8)$ \\
\hline Non-functional PanNET, n (\%) & $18(90)$ \\
\hline Insulinoma, $\mathrm{n}$ & 2 \\
\hline Sporadic, n (\%) & $16(80)$ \\
\hline MEN1, $\mathrm{n}$ & 2 \\
\hline TSC, $\mathrm{n}$ & 2 \\
\hline EUS FNA, n (\%) & $15(75)$ \\
\hline EUS FNB, $n$ & 1 \\
\hline EUS not specified, $\mathrm{n}$ & 4 \\
\hline Location Head, n (\%) & $5(25)$ \\
\hline Location Body, n (\%) & $3(15)$ \\
\hline Location Tail, n (\%) & $12(60)$ \\
\hline Resection primary tumor, $\mathrm{n}$ & 13 \\
\hline - $\quad$ Size \pm SD $(\mathrm{cm})$ & $5.2(6.2)$ \\
\hline - $\quad T 1, n(\%$ of resected) & $8(62)$ \\
\hline - $\quad \mathrm{T} 2, \mathrm{n}(\%$ of resected) & $5(38)$ \\
\hline - $\quad$ Radical, $\mathrm{n}(\%)$ & $9(69)$ \\
\hline - $\quad$ + Lymph nodes, $\mathrm{n}(\%)$ & $5(38)$ \\
\hline - $\quad$ - Lymph nodes, n (\%) & $6(46)$ \\
\hline - $\quad$ No nodes reported, $n$ & $2(15)$ \\
\hline No resection & 7 \\
\hline - $\quad$ Size $\pm S D(\mathrm{~cm})$ & $1.7(0.7)$ \\
\hline Liver metastasis, n (\%) & $5(25)$ \\
\hline
\end{tabular}
ne neoplasia 1; TSC, tuberous sclerosis complex; EUS, endoscopic ultrasound; FNA/B, fine needle aspiration/biopsy.

pathology.jhmi.edu/telometer) and analyzed as previously described. For the cytologic specimens, ultrabright foci were not confirmed with Telometer due to common high background fluorescence. Centromeric FISH signals were used to exclude non-specific foci and as positive hybridization control. If less than 100 cells could be counted in the cytology by the automated Ki67 count or no ultrabright foci were observed, cases were called non informative.

\section{Statistics}

Data was analyzed in SPSS version 25 (IBM Nederland, Amsterdam, The Netherlands). To determine inter-observer agreement, the percentage of agreement and the Kappa value were calculated. For comparisons between matched samples (cytologic and surgical specimens) the percentage of concordance, sensitivity, specificity and the Kappa value were calculated. To assess prognostic value, dichotomous comparisons were analyzed using Fisher's exact test. Continuous comparisons like Ki67 labeling index were made using the Wilcoxon signed rank test (17). 


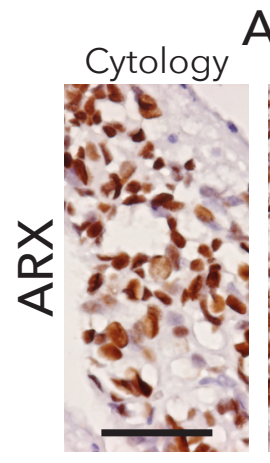

A B
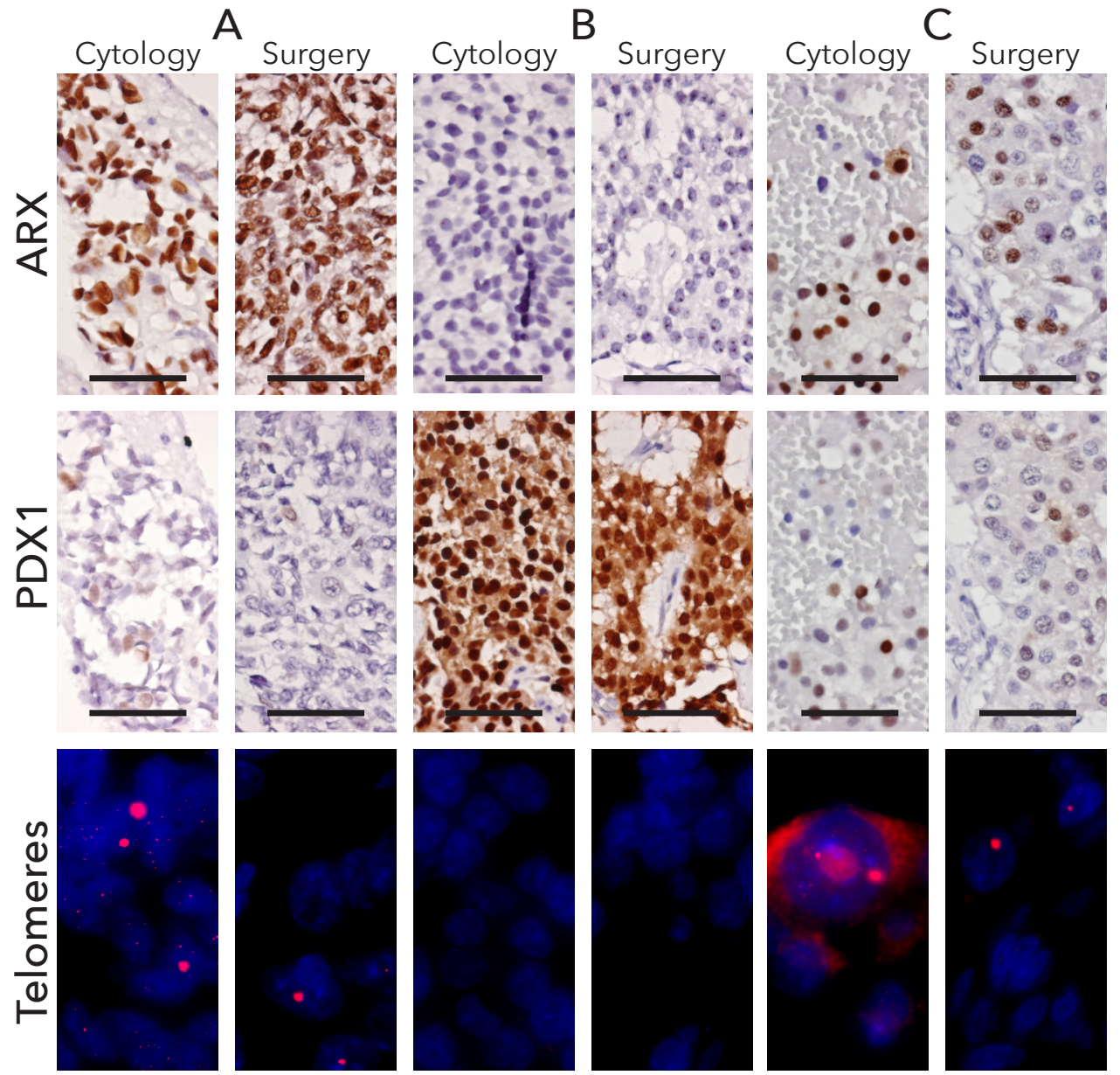

Figure 1. Immunohistochemistry and telomere-specific fluorescence in situ hybridization of cytologic and surgical specimens. Representative images of cytologic and surgical specimens. A, patient 13; ARX positive, PDX1 negative tumor, ALT positive. B, patient 6; ARX negative, PDX1 positive tumor, without ALT. C, patient 10, discordant case for PDX1 expression; ARX positive, PDX1 negative, ALT positive surgical specimen. PDX1 positive cells are present in the surgical specimen but are below the defined cut-off. Also note the cytoplasmic background fluorescence in the telomere FISH cytologic specimen. IHC at 40x magnification, 50 $\mathrm{mm}$ scale bar. Telomere FISH at 100x, nucleus visible as DAPI blue and ultrabright telomeric signals in the red channel.

\section{RESULTS}

\section{Patient and material characteristics}

Endoscopic fine needle aspiration cellblock material from 20 patients with a PanNET diagnosis, 13 of which had corresponding surgical specimens, was included (table 1). 14 cytologic specimens were formalin fixed, agar-gelatin or paraffin embedded blocks, the 


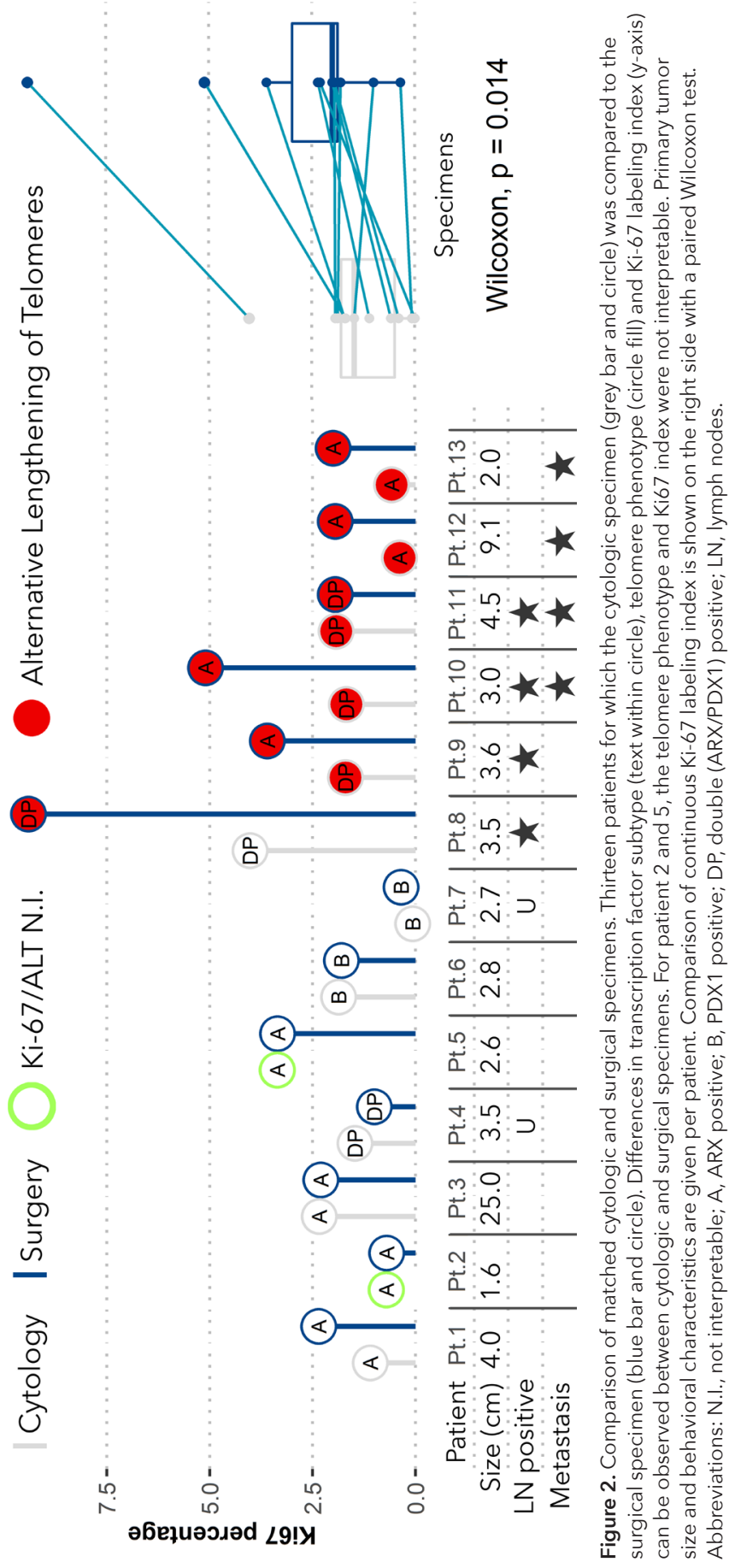


Table 2. Kappa coefficients of comparison surgical and cytologic specimens for prognostic markers

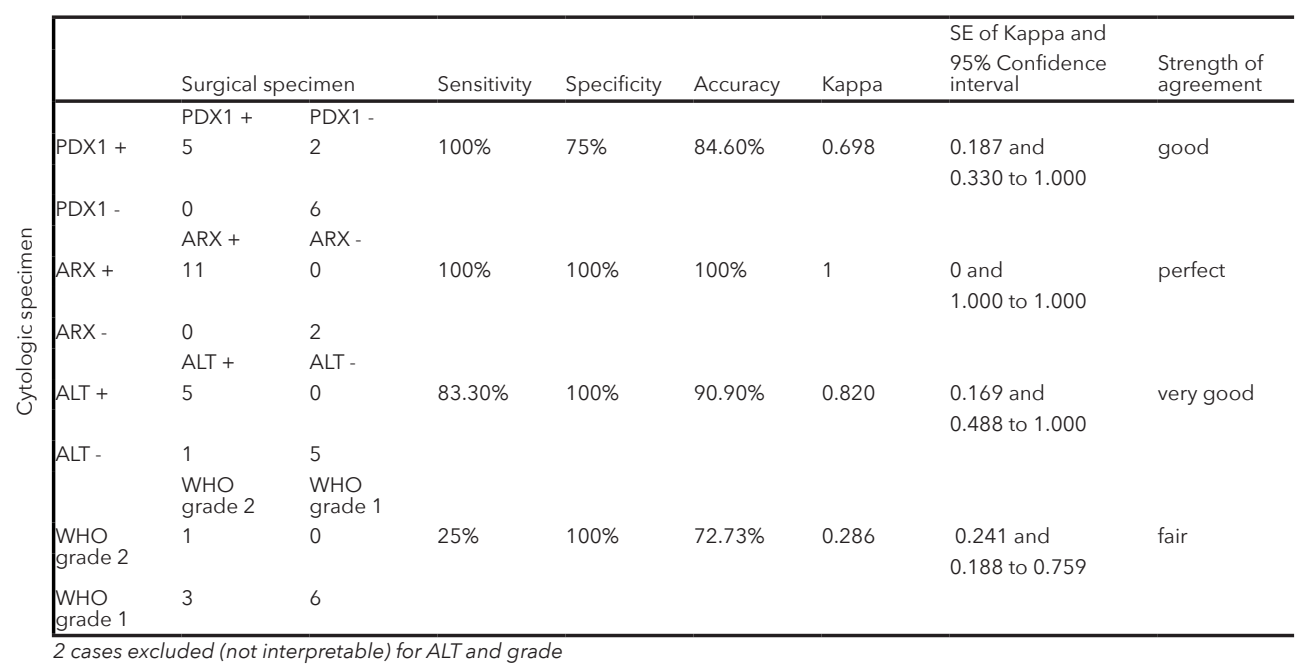

remainder was fixed with Cytolyt and subsequently with formalin. One case was Cytolyt fixed and embedded as Cellient (paraffin) block. All surgical specimens were FFPE blocks.

\section{Cytologic versus surgical specimens}

Overall, using a representative surgical specimen tumor block as reference standard, $85 \%$ of the cytology cases (11/13 correct) were accurate in determining tumor transcription factor subtype (ARX+/PDX1-, ARX-/PDX1+, ARX+/PDX1+, ARX-/PDX1-)(Fig 1). Concordance of cytologic and surgical specimens for ARX and PDX1 IHC, ALT status, and WHO tumor grade was 100\% (Kappa $=1)$, 85\% (Kappa $=0.70), 91 \%($ Kappa $=0.82)$ and $73 \%($ Kappa $=0.29$ ), while the sensitivity and specificity were 100\%/100\%, 100\%/75\%,83\%/100\% and $25 \% / 100 \%$, respectively (table 2 ). Two ARX+/PDX1- cases in the surgical specimens were scored as ARX+/PDX1+ in the cytology specimens. In these cases, additional tumor blocks from the surgical specimen were stained to exclude intratumoral heterogeneity often seen in larger tumors which could explain the discrepancy, but the size was average (3.0 and 3.6 $\mathrm{cm}$ ) and no demarcated heterogeneous expression was noted. Both surgical specimens approached the defined cut-offs for positive expression. Focal areas of more than $50 \%$ of weakly positive cells were found, as well as areas with few strong positive cells, however these fell just below the defined cut-offs for the whole slide (Fig 1C). Also, one PDX1 negative surgical specimen was surrounded by stroma with many strong positive PDX1 positive 
islets of Langerhans (which would have been synaptophysin positive in the cytology). In both discrepant cases, more than 500 cells were present in the cytologic specimens. In one case (patient 11), both observers noted heterogeneous (patchy) positive PDX1 expression in the primary tumor, which was also independently noted when scoring the corresponding cytologic specimen. All other cases showed homogeneous protein expression throughout the tumor.

One case with ALT was missed by cytology. For this case 498 cells were counted in the cytology. Although one possible ultrabright focus was found in this cytologic specimen, it was below the $1 \%$ cutoff. Only one out of four WHO grade 2 tumors were correctly identified by cytology, the continuous Ki67 labeling index in cytology significantly underestimated the percentage of Ki67 positive cells compared to the surgical specimen. (Wilcoxon $\mathrm{P}=$ 0.014, Fig 2). Concordance was not better in smaller tumors or cytologic specimens with higher cell counts.

\section{Cytology methods, inter-observer agreement}

There were no clear variations in immunogenicity for IHC observed between different fixatives, FNA or FNB. Both discrepant cases were agar FFPE (2/13). For the single Cellient block material, the Ki67 percentage count failed material due to loss of morphology and lack of cell clusters, and the telomere FISH was not interpretable due to hybridization failure. There was perfect inter-observer agreement in scoring the cytology cases and the primary tumors for ARX and PDX1 (Kappa 1).

\section{Clinical behavior}

Of 20 patients, 5 developed liver metastases during follow-up (supplementary table 1). Marker expression was preferably determined in the surgical specimens, but in cytologic specimens if surgery was not performed. All metastatic tumors expressed ARX, two also expressed PDX1 (double positive). In the non-metastatic tumors 12 out of 15 had ARX expression of which 2 were double positive, while 2 tumors had sole PDX1 expression and 1 tumor had no ARX or PDX1 expression (double negative) (supplementary table 1). Mean size was not significantly different in metastatic versus non-metastatic cases (4.3 vs $3.9 \mathrm{~cm}$ ). Only ALT positivity was significantly associated with liver metastases (Fisher's exact $p=0.0014)$. 


\section{DISCUSSION}

ARX expression was found to be perfectly concordant between pre-operative cytologic specimens and post-operative surgical specimens and there was no inter-observer variation. ALT was confirmed to be a reliable marker with very good concordance between cytologic and surgical specimens (11). Furthermore, the presence of ALT was the best predictor of metastatic tumor behavior. Most cytologic specimens were correctly classified as normal or ALT, but one ALT positive case was missed. PDX1 showed a fair concordance between cytologic and surgical specimens, but did not identify indolent tumors in this small cohort (12). PDX1 expression has been described before in the metastatic setting (18). Furthermore, in contrast to ARX, PDX1 is expressed in pancreatic acinar and ductal cells (supplementary Fig 1), and more abundantly in duodenum and islets of Langerhans, causing potential false positive PDX1 staining due to contamination - a possible explanation for the discrepancies in this study. Also, focal variation in staining intensity and percentage together with scoring cut-offs make reliable interpretation of PDX1 on cytology more prone to error. Since reliable cytologic determination of PDX1 is critical to determine the prognostic subgroups as described in Cejas et al., cytologic PDX1 interpretation should be performed with caution, but concerning prognostic value, this study was too limited in size to draw strong conclusions. The high percentage of ARX positive non-functioning PanNETs $(17 / 18)$ compared to $50-60 \%$ in recent studies $(12,13)$, might be caused by selection bias and reflect more aggressive/faster growing tumors that warranted biopsy.

Ki67 count in cytologic specimens generally underestimated Ki67 count as seen in corresponding surgical specimens (Fig 2). This led to three grade 2 tumors to be erroneously classified as grade 1 tumors based on the Ki67 count in the cytologic specimen. Difficulties of correctly diagnosing intermediate grade or grade 2 PanNETs in cytology have often been reported and usually lead to an underestimation of grade (19-25). It has been shown that accuracy decreases with larger tumors (with possible heterogeneity) and paucicellular cytologic specimens, but this was not seen in the current study $(21,24,26,27)$.

The use of synaptophysin stained slides, annotated for areas with clusters of NET cells proved to be very useful and time saving in maximizing the cell count from cytologic specimens, without losing specificity (e.g. lymphocytes or reactive tissue can have Ki67 positivity, and necrotic debris and blood often have non-specific binding fluorescent foci which need to be manually check to be excluded). Theoretically, a multiplex/double synaptophysin 
immunofluorescence staining and telomere FISH would have been even more accurate, but this was not feasible due to intense (cytoplasmic) background fluorescence often observed in the cytologic specimens (Fig 1C). Nevertheless, our methodology of using consecutive slides was sufficient as the clusters of cells were always identified exactly as on the annotated slides.

While 12/13 cases were concordant between cytology and the resection for ALT status, one ALT positive case was missed on cytology, which can be explained by the definition of the cut-off to call ALT (>1\%). In fact, one ultrabright focus was observed in this false negative cytologic specimen (1/498), while not a single focus was found in any of the cytologic specimens of the true ALT negative cases. It might therefore be better to use a higher cutoff (e.g. 1000 cells) to determine if a sample has normal telomeres instead of the 100 cells used in this study, or to call ALT positivity in case of any focus is seen in cytology, irrespective of percentage, as these foci appear to be very specific for ALT in cytology.

To conclude, ALT (detected by telomere FISH) and ARX protein expression (detected by $\mathrm{IHC}$ ) can reliably be determined in cytologic specimens, irrespective of aspiration or most fixation methods. In contrast, PDX1 is less attractive for pre-operative use. Ki67 count in cytology frequently underestimates tumor grade and should therefore be interpreted with great caution. Further validation of ARX and PDX1 protein expression in larger retrospective cohorts should be performed to definitively determine their true prognostic value while prospective pre-operative validation of ALT as a biomarker in PanNET is warranted.

\section{CONFLICTS OF INTEREST:}

None

\section{ACKNOWLEDGMENTS}

We thank T. Peeters and A. Meeker for their help with setting up the fluorescence in situ hybridization assay. Maag Darm Lever Stichting (Dutch Digestive Foundation) CDG 14-020

\section{REFERENCES}

1. Keutgen XM, Schadde E, Pommier RF, Halfdanarson TR, Howe JR, Kebebew E. Metastatic neuroendocrine tumors of the gastrointestinal tract and pancreas: A surgeon's plea to centering attention on the liver. Semin Oncol. 2018;45(4):232-5.

2. vila J, Caplin M, et al. ENETS Consensus Guidelines Update for the
Management of Patients with Functional Pancreatic Neuroendocrine Tumors and Non-Functional Pancreatic Neuroendocrine Tumors. Neuroendocrinology. 2016;103(2):153-71.

3. Kunz PL, Reidy-Lagunes D, Anthony LB, Bertino EM, Brendtro K, Chan JA, et al. Consensus guidelines for the management and treatment of neuroendocrine tumors. Pancreas. 


\section{3;42(4):557-77.}

4. Marinoni I, Kurrer AS, Vassella E, Dettmer M, Rudolph T, Banz V, et al. Loss of DAXX and ATRX are associated with chromosome instability and reduced survival of patients with pancreatic neuroendocrine tumors. Gastroenterology. 2014;146(2):453-60 e5.

5. Singhi AD, Liu TC, Roncaioli JL, Cao D, Zeh HJ,

Zureikat AH, et al. Alternative Lengthening of Telomeres and Loss of DAXX/ATRX Expression Predicts Metastatic Disease and Poor Survival in Patients with Pancreatic Neuroendocrine Tumors. Clin Cancer Res. 2017;23(2):600-9.

6. Kim JY, Brosnan-Cashman JA, An S, Kim SJ, Song

$\mathrm{KB}$, Kim MS, et al. Alternative Lengthening of Telomeres in Primary Pancreatic Neuroendocrine Tumors Is Associated with Aggressive Clinical Behavior and Poor Survival. Clin Cancer Res. 2017;23(6):1598-606.

7. Chou A, Itchins M, de Reuver PR, Arena J, Clarkson $A$, Sheen A, et al. ATRX loss is an independent predictor of poor survival in pancreatic neuroendocrine tumors. Hum Pathol. 2018;82:249-57

8. Park JK, Paik WH, Lee K, Ryu JK, Lee SH, Kim YT. DAXX/ATRX and MEN1 genes are strong prognostic markers in pancreatic neuroendocrine tumors. Oncotarget. 2017;8(30):49796-806.

9. Heaphy CM, de Wilde RF, Jiao Y, Klein AP, Edil BH, Shi $C$, et al. Altered telomeres in tumors with ATRX and DAXX mutations. Science. 2011;333(6041):425.

10. Hechtman JF, Klimstra DS, Nanjangud G, Frosina D,

Shia J, Jungbluth AA. Performance of DAXX Immunohistochemistry as a Screen for DAXX Mutations in Pancreatic Neuroendocrine Tumors. Pancreas. 2019;48(3):396-9.

11. VandenBussche CJ, Allison DB, Graham MK, Charu $\mathrm{V}$, Lennon $A M$, Wolfgang $C L$, et al. Alternative lengthening of telomeres and ATRX/DAXX loss can be reliably detected in FNAs of pancreatic neuroendocrine tumors. Cancer Cytopathol. 2017;125(7):544-51.

12. Cejas P, Drier Y, Dreijerink KMA, Brosens LAA, Deshpande $V$, Epstein $C B$, et al. Enhancer signatures stratify and predict outcomes of non-functional pancreatic neuroendocrine tumors. Nat Med. 2019;25(8):1260-5.

13. Chan CS, Laddha SV, Lewis PW, Koletsky MS, Robzyk K, Da Silva E, et al. ATRX, DAXX or MEN1 mutant pancreatic neuroendocrine tumors are a distinct alpha-cell signature subgroup. Nat Commun. 2018;9(1):4158.

14. Cesare AJ, Heaphy CM, O'Sullivan RJ. Visualization of Telomere Integrity and Function In Vitro and In Vivo Using Immunofluorescence Techniques. Curr Protoc Cytom. 2015;73:12 $401-31$.

15. Conemans EB, Brosens LAA, Raicu-lonita GM, Pieterman CRC, de Herder WW, Dekkers OM, et al. Prognostic value of WHO grade in pancreatic neuro-endocrine tumors in Multiple Endocrine Neoplasia type 1: Results from the DutchMEN1 Study Group. Pancreatology. 2017;17(5):766-72.

16. Inzani F, Petrone G, Rindi G. The New World Health Organization Classification for Pancreatic Neuroendocrine Neoplasia. Endocrinol Metab Clin North Am. 2018;47(3):463-70.

17. Carlinfante G, Baccarini P, Berretti D, Cassetti T, Cavina $\mathrm{M}$, Conigliaro $\mathrm{R}$, et al. Ki-67 cytological index can distinguish well-differentiated from poorly differentiated pancreatic neuroendocrine tumors: a comparative cytohistological study of 53 cases. Virchows Arch. 2014;465(1):49-55.

18. Yang Z, Klimstra DS, Hruban RH, Tang LH. Immunohistochemical Characterization of the Origins of Metastatic Well-differentiated Neuroendocrine Tumors to the Liver. Am J Surg Pathol. 2017;41(7):915-22.

19. Weynand B, Borbath I, Bernard V, Sempoux C, Gigot JF, Hubert $C$, et al. Pancreatic neuroendocrine tumour grading on endoscopic ultrasound-guided fine needle aspiration: high reproducibility and inter-observer agreement of the Ki-67 labelling index. Cytopathology. 2014;25(6):389-95.

20. Rebours V, Cordova J, Couvelard A, Fabre M, Palazzo $\mathrm{L}$, Vullierme MP, et al. Can pancreatic neuroendocrine tumour biopsy accurately determine pathological characteristics? Dig Liver Dis. 2015;47(11):973-7.

21. Fujimori N, Osoegawa T, Lee L, Tachibana Y, Aso A, Kubo $\mathrm{H}$, et al. Efficacy of endoscopic ultrasonography and endoscopic ultrasonography-guided fine-needle aspiration for the diagnosis and grading of pancreatic neuroendocrine tumors. Scand J Gastroenterol. 2016;51(2):245-52.

22. Diaz Del Arco C, Diaz Perez JA, Ortega Medina L, Sastre Valera J, Fernandez Acenero MJ. Reliability of Ki-67 Determination in FNA Samples for Grading Pancreatic Neuroendocrine Tumors. Endocr Pathol. 2016;27(4):276-83.

23. Hwang HS, Kim Y, An S, Kim SJ, Kim JY, Kim SY, et al. Grading by the Ki-67 Labeling Index of Endoscopic Ultrasound-Guided Fine Needle Aspiration Biopsy Specimens of Pancreatic Neuroendocrine Tumors Can Be Underestimated. Pancreas. 2018;47(10):1296-303.

24. Boutsen L, Jouret-Mourin A, Borbath I, van Maanen A, Weynand B. Accuracy of Pancreatic Neuroendocrine Tumour Grading by Endoscopic Ultrasound-Guided Fine Needle Aspiration: Analysis of a Large Cohort and Perspectives for Improvement. Neuroendocrinology. 2018;106(2):158-66.

25. Cui Y, Khanna LG, Saqi A, Crapanzano JP, Mitchell JM, Sethi A, et al. The Role of Endoscopic Ultrasound-Guided Ki67 in

The Management of Non-Functioning Pancreatic Neuroendocrine Tumors. Clin Endosc. 2019.

26. Hasegawa T, Yamao K, Hijioka S, Bhatia V, Mizuno N, Hara K, et al. Evaluation of Ki-67 index in EUS-FNA specimens for the assessment of malignancy risk in pancreatic neuroendocrine tumors. Endoscopy. 2014;46(1):32-8.

27. Unno J, Kanno A, Masamune A, Kasajima A, Fujishima $F$, Ishida $K$, et al. The usefulness of endoscopic ultrasound-guided fine-needle aspiration for the diagnosis of pancreatic neuroendocrine tumors based on the World Health Organization classification. Scand J Gastroenterol. 2014;49(11):1367-74. 


\section{SUPPLEMENTARY MATERIALS}

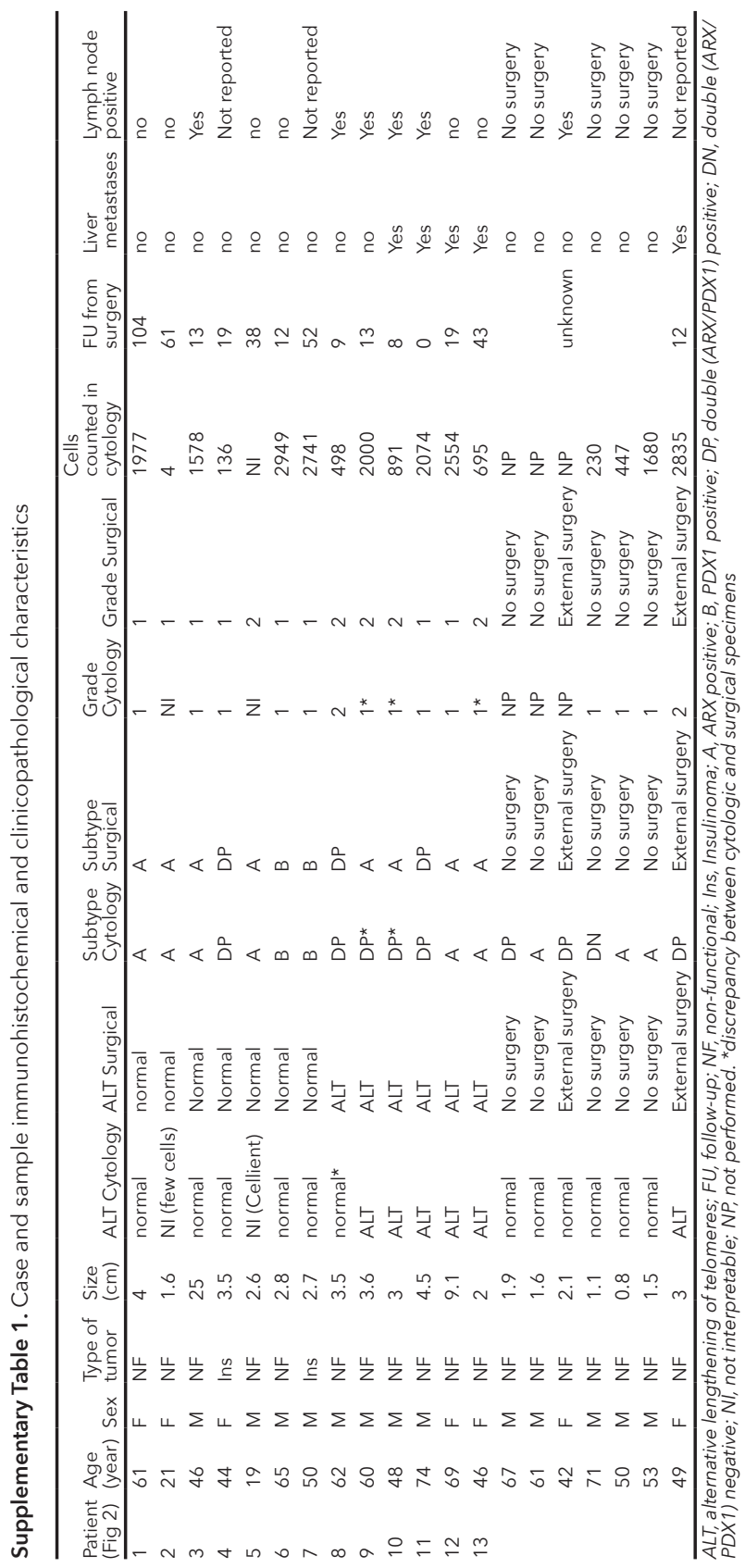




\section{PDX1}

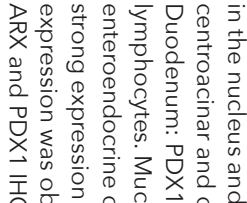

둥ํำ

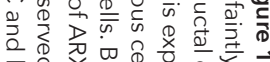

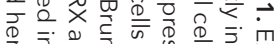

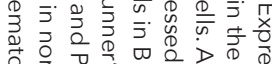

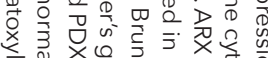

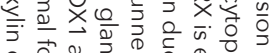
กิ

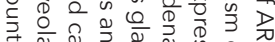

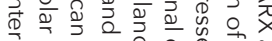

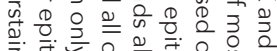

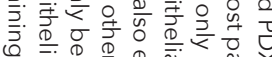

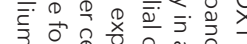

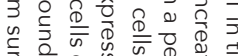

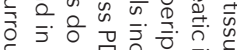
ว 윽

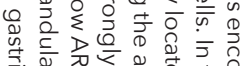

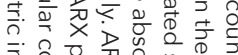

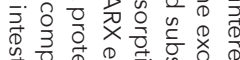

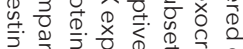

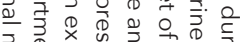
उ. 责年出.

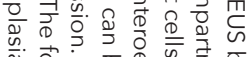

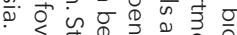

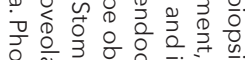

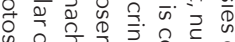

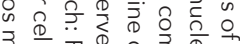

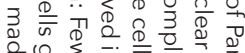

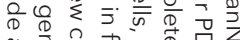

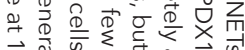

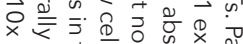

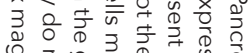
늘.

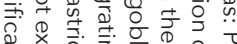

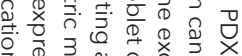

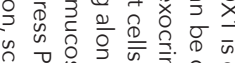

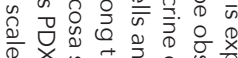

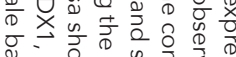

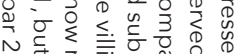
다. $3 \equiv 3 \stackrel{2}{2}$

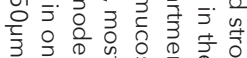

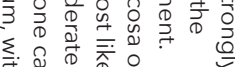
垨食南交
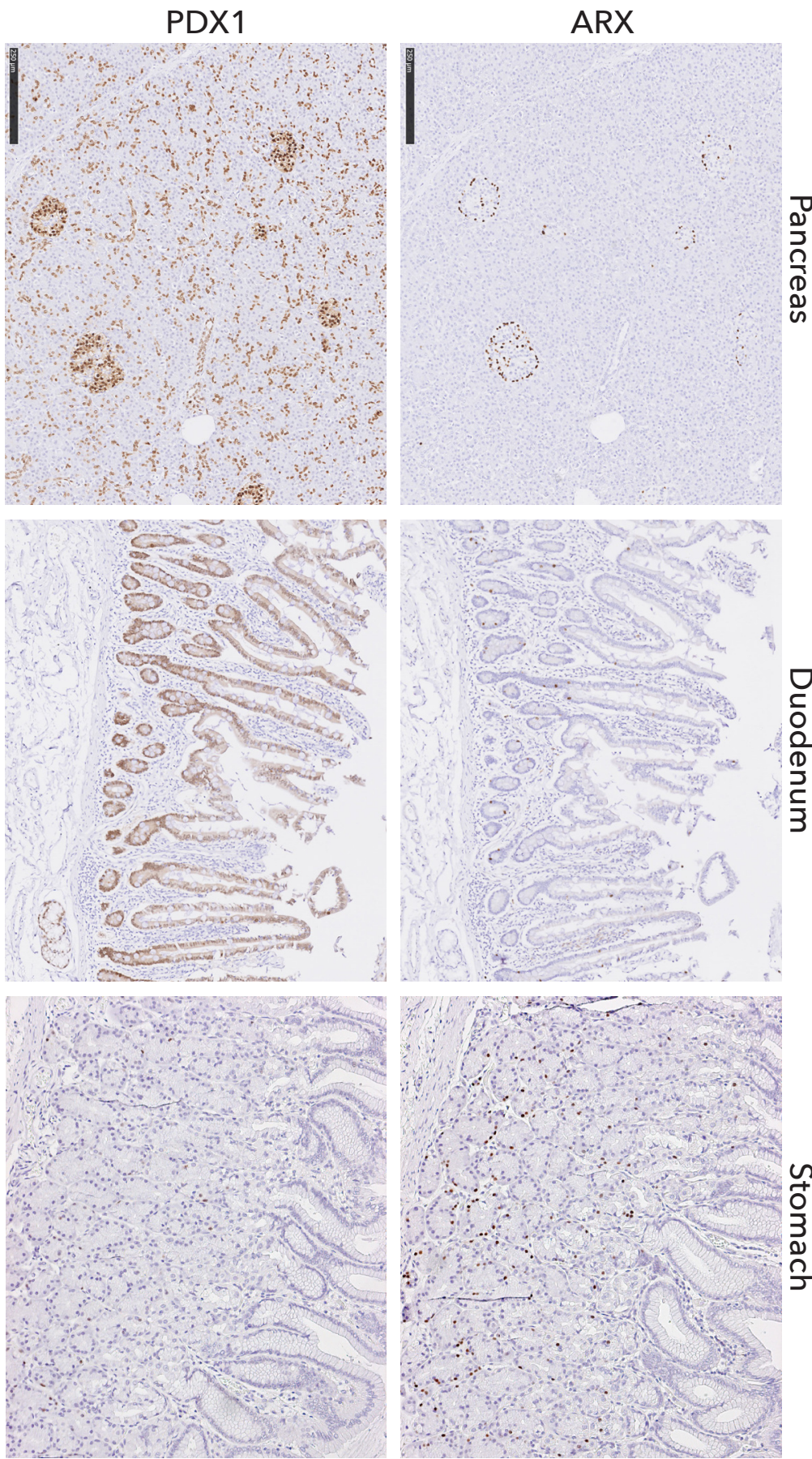

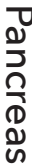

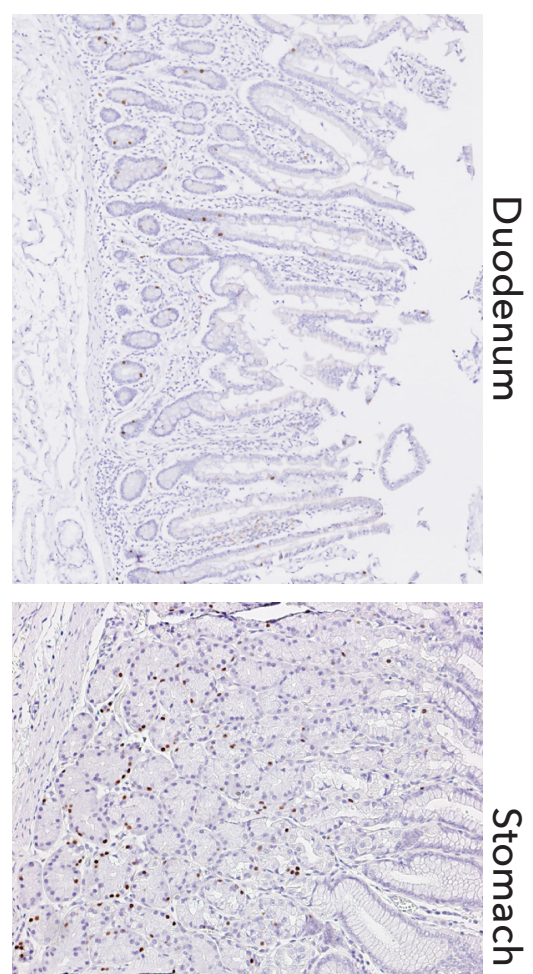



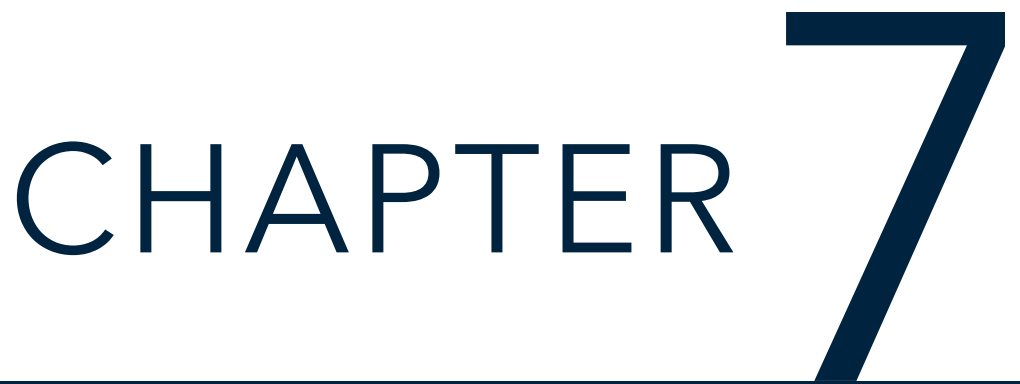

Alternative Lengthening of Telomeres and Differential Expression of Endocrine Transcription Factors Distinguish Metastatic and Nonmetastatic Insulinomas

Wenzel M. Hackeng, Willemien Schelhaas, Folkert H.M. Morsink, Charlotte M. Heidsma, Susanne van Eeden, Gerlof D. Valk, Menno R. Vriens, Christopher M. Heaphy, Els J.M. Nieveen van Dijkum, G. Johan A. Offerhaus, Koen M.A. Dreijerink, Lodewijk A.A. Brosens 


\section{ABSTRACT}

Insulin-producing pancreatic neuroendocrine tumors (PanNETs)/insulinomas are generally considered to be indolent tumors with an excellent prognosis after complete resection. However, some insulinomas have a poor prognosis due to relapses and metastatic disease. Recently, studies in non-functional PanNETs indicated that behavior can be stratified according to alpha- and beta-cell differentiation, as defined by expression of the transcription factors ARX and PDX1, respectively. It is unknown whether similar mechanisms play a role in insulinomas. Therefore, we determined ARX and PDX1 expression in a cohort of 35 sporadic primary insulinomas and 2 liver metastases of inoperable primary insulinomas. In addition, WHO-grade and loss of ATRX or DAXX were determined by immunohistochemistry, and alternative lengthening of telomeres (ALT) and CDKN2A status by fluorescence in situ hybridization. These findings were correlated with tumor characteristics and clinical follow-up data.

In total, 5 out of 37 insulinoma patients developed metastatic disease. Metastatic insulinomas were all larger than $3 \mathrm{~cm}$, whereas the indolent insulinomas were smaller ( $p$-value $<0.05)$. All three primary insulinomas that metastasized showed ARX expression, 2/3 showed ALT, and $1 / 3$ had a homozygous deletion of CDKN2A as opposed to absence of ARX expression, ALT, or CDKN2A deletions in the 32 nonmetastatic cases. The two liver metastases also showed ARX expression and ALT (2/2). The presence of ARX expression, which is usually absent in beta-cells, and genetic alterations not seen in indolent insulinomas strongly suggest a distinct tumorigenic mechanism in malignant insulinomas, with similarities to non-functional PanNETs. These observations may inform future follow-up strategies after insulinoma surgery. 


\section{INTRODUCTION}

Insulinomas are the most common functional pancreatic neuroendocrine tumor (PanNET) type and are diagnosed by the triad of hypoglycemic symptoms, low blood glucose concentrations, and relief of symptoms after glucose administration. Most insulinomas are indolent tumors: there are few mitoses (low grade) and metastases are very rare (1, 2). In contrast, $40-50 \%$ of non-functional PanNETs present with liver metastases at time of initial diagnosis $(3,4)$. Surgery for insulinomas is primarily indicated to alleviate symptoms of hypoglycemia. Survival after surgery is not different from the general population $(5,6)$. There are no insulinoma-specific international recommendations for follow-up after surgery $(5,7)$. Nevertheless, about $10 \%$ of insulinoma patients develop metastases that are mostly present at time of initial diagnosis, but may sometimes develop years after resection of the primary tumor (8). Median survival is less than 2 years in patients with metastatic insulinoma, similar to metastatic non-functional PanNET (9-11).

Because of the rarity of metastatic insulinomas little is known about the mechanisms of tumorigenesis. Recent sequencing studies identified recurrent $Y Y 1$ gene mutations in insulinomas; however relapses or metastases were rarely reported in the studied cohorts (12-15). There is a need to increase our understanding of insulinoma development, in particular of metastatic insulinomas. This will improve identification of patients at risk for recurrence, who may benefit from follow-up after surgery and thereby earlier detection and treatment of metastases.

Metastatic non-functional PanNETs are more common and have been characterized more extensively. Next-generation sequencing studies have demonstrated that sporadic PanNETs harbor relatively few gene mutations. The genes that are most frequently mutated are MEN1, ATRX and DAXX $(12,16,17)$. Mutually exclusive mutations in ATRX or DAXX - coding for two chromatin modifying proteins which form a histone chaperone complex - are associated with the alternative lengthening of telomeres phenotype (ALT) (18). Immunohistochemical loss of ATRX and DAXX can be used as a surrogate marker of inactivating mutations of ATRX or $D A X X$, respectively $(18,19)$. The presence of either of these alterations is associated with recurrence and liver metastases (20-25). In addition to ATRX or DAXX mutations and ALT, loss of ARID1A, loss of H3K36 trimethylation (H3K36me3) by SETD2 function dysfunction, and CDKN2A deletions were also reported to be of prognostic value for non-functional PanNET (26). A recent finding in non-functional PanNETs is that alpha- or beta-cell types-of- 
origin may predict clinical behavior $(20,27)$. Beta-cell like non-functional PanNETs, marked by expression of the endocrine transcription factor PDX1, were generally indolent, while almost all relapses were observed in the group of alpha-cell like non-functional PanNETs marked by ARX expression. Furthermore, somatic mutations in ATRX, DAXX, and MEN1 and acquisition of ALT were observed more often in the alpha-type non-functional PanNETs (20, 27).

Whether the ARX and PDX1 transcription factors play a role in the clinical behavior of insulinomas is not known. We determined protein expression of ARX and PDX1 together with ATRX, DAXX, ARID1A, and H3K36me3 by immunohistochemistry, as well as ALT and CDKN2A deletions by fluorescence in situ hybridization (FISH), in a cohort of clinically defined sporadic insulinomas.

\section{MATERIALS AND METHODS}

\section{Study Cohort}

The study was approved by the University Medical Center (UMC) Utrecht Biobank Research Ethics Committee. Tissue-microarrays (TMAs) were constructed of primary sporadic insulinomas resected between 1991 - 2017 and 1997 - 2017 for the UMC Utrecht and Amsterdam UMC, respectively. Inherited cases (e.g. MEN1 syndrome) were excluded. Neuroendocrine tumor diagnosis was confirmed by an experienced gastrointestinal pathologist ( $L A A B)$. Three $0.6 \mathrm{~mm}$ cores per tumor were randomly taken from annotated tumor areas in formalin-fixed paraffin-embedded (FFPE) blocks. In case of multiple tumors, the largest tumor was used. Biopsies of insulinoma liver metastases were identified by a search in the UMC Utrecht pathology archive.

Information on age, sex, multifocality, surgery type, surgery date, tumor size, location, grade, resection margin and lymph nodes was collected from pathology reports. If possible, macroscopic tumor size was used. Free margins were interpreted as R0, also if the distance was less than $1 \mathrm{~mm}$ from the resection margin. Medical records were reviewed to collect information on the functional status of the tumor, presence of genetic syndromes, and follow-up. Events of tumor relapse (local recurrence, liver metastases, or other metastases) were either histologically proven or diagnosed by the treating clinician. The first radiological evidence of proven relapse was used as event time point. Follow-up time is counted from date of surgery until described events, death, or was censored at the last visit to a relevant hospital clinician (surgery, internal medicine, endocrinology, gastroenterology, or 
Table 1. Antibodies and protocol variations

\begin{tabular}{|c|c|c|c|c|c|c|c|}
\hline $\begin{array}{l}\text { Antibody } \\
\text { target }\end{array}$ & Company & Name & $\begin{array}{l}\text { Species and } \\
\text { (clone) }\end{array}$ & $\begin{array}{l}\text { Pre-treat- } \\
\text { ment }\end{array}$ & $\begin{array}{l}\text { Dilution / Time / } \\
\text { Temperature }\end{array}$ & Substrate & Scoring Method \\
\hline$\overline{\mathrm{DAXX}}$ & $\begin{array}{l}\text { Atlas antibo- } \\
\text { dies, Bromma, } \\
\text { Sweden }\end{array}$ & HPA008736 & Rabbit PAB & $\begin{array}{l}\text { ARS/pH6 } \\
20 \text { min. }\end{array}$ & 1:100 1 hour RT & Bright-DAB & $\begin{array}{l}\text { Negative if positive nuclear } \\
\text { staining }<5 \% \text { of tumor cells }\end{array}$ \\
\hline ATRX & $\begin{array}{l}\text { Sigma, St. Louis, } \\
\text { MO }\end{array}$ & HPA0001906 & Rabbit PAB & $\begin{array}{l}\text { ARS/pH9 } \\
20 \mathrm{~min}\end{array}$ & $\begin{array}{l}\text { 1:400 overnight } \\
4^{\circ} \mathrm{C}\end{array}$ & $\mathrm{DAB}$ & $\begin{array}{l}\text { Negative if positive nuclear } \\
\text { staining }<5 \% \text { of tumor cells }\end{array}$ \\
\hline ARX & $\begin{array}{l}\text { Millipore, Bur- } \\
\text { lington, MA }\end{array}$ & MABN102 & $\begin{array}{l}\text { Mouse MAB } \\
(11 \mathrm{~F} 6.2)\end{array}$ & $\begin{array}{l}\text { ARS/pH6 } \\
20 \mathrm{~min} .\end{array}$ & $\begin{array}{l}\text { 1:2000 } 1 \text { hour } \\
\text { RT }\end{array}$ & DAB & $\begin{array}{l}\text { Positive if weak nuclear } \\
\text { staining }>50 \% \text { or intermedi- } \\
\text { ate/strong nuclear staining } \\
>10 \% \text { of tumor cells }\end{array}$ \\
\hline PDX1 & $\begin{array}{l}\text { Abcam, Cam- } \\
\text { bridge, United } \\
\text { Kingdom }\end{array}$ & $a b 134150$ & $\begin{array}{l}\text { Rabbit MAB } \\
\text { (EPR3358(2)) }\end{array}$ & $\begin{array}{l}\text { ARS/pH6 } \\
20 \mathrm{~min} .\end{array}$ & $\begin{array}{l}\text { 1:2000 } 1 \text { hour } \\
\text { RT }\end{array}$ & DAB & $\begin{array}{l}\text { Positive if weak nuclear } \\
\text { staining }>50 \% \text { or intermedi- } \\
\text { ate/strong nuclear staining } \\
>10 \% \text { of tumor cells }\end{array}$ \\
\hline Ki67 & $\begin{array}{l}\text { Immunologic, } \\
\text { Duiven, The } \\
\text { Netherlands }\end{array}$ & $\begin{array}{l}\text { VWR- } \\
\text { KILM9252-C05 }\end{array}$ & $\begin{array}{l}\text { Mouse MAB } \\
\text { (MIB1) }\end{array}$ & $\begin{array}{l}\text { ARS /pH6 } \\
20 \text { min. }\end{array}$ & 1:200 1 hour RT & DAB & $\begin{array}{l}\text { Digital image analysis of } \\
\text { nuclear expression in at } \\
\text { least } 2000 \text { tumor cells }\end{array}$ \\
\hline Glucagon & $\begin{array}{l}\text { Cell Marque, } \\
\text { Rocklin, CA }\end{array}$ & 259A-15 & Rabbit PAB & $\begin{array}{l}\text { ARS } / \mathrm{pH} 6 \\
20 \mathrm{~min} .\end{array}$ & 1:100 1 hour RT & Bright-DAB & $\begin{array}{l}\text { Positive if cytoplasmic } \\
\text { staining }>10 \% \text { of tumor } \\
\text { cells, scattered if }<10 \% \text { of } \\
\text { tumor cells }\end{array}$ \\
\hline Insulin & $\begin{array}{l}\text { Dako, Santa } \\
\text { Clara, CA }\end{array}$ & A564 & Rabbit PAB & $\begin{array}{l}\text { ARS } / \mathrm{pH} 6 \\
20 \mathrm{~min} .\end{array}$ & 1:100 1 hour RT & DAB & $\begin{array}{l}\text { Positive if cytoplasmic } \\
\text { staining }>10 \% \text { of tumor } \\
\text { cells, scattered if }<10 \% \text { of } \\
\text { tumor cells }\end{array}$ \\
\hline $\begin{array}{l}\text { Histone } \\
\text { H3K- } \\
36 \mathrm{me} 3\end{array}$ & $\begin{array}{l}\text { Abcam, Cam- } \\
\text { bridge, United } \\
\text { Kingdom }\end{array}$ & $a b 9050$ & Rabbit PAB & $\begin{array}{l}\text { ARS } / \mathrm{pH} 6 \\
20 \text { min. }\end{array}$ & $\begin{array}{l}\text { 1:2000 } 1 \text { hour } \\
\text { RT }\end{array}$ & DAB & $\begin{array}{l}\text { Negative if positive nuclear } \\
\text { staining }<30 \% \text { of tumor cells }\end{array}$ \\
\hline ARID1A & $\begin{array}{l}\text { Abcam, Cam- } \\
\text { bridge, United } \\
\text { Kingdom }\end{array}$ & $a b 182560$ & $\begin{array}{l}\text { Rabbit MAB } \\
\text { (EPR13501) }\end{array}$ & $\begin{array}{l}\text { ARS /pH6 } \\
20 \text { min. }\end{array}$ & $\begin{array}{l}\text { 1:1000 } 1 \text { hour } \\
\text { RT }\end{array}$ & DAB & $\begin{array}{l}\text { Negative if positive nuclear } \\
\text { staining }<5 \% \text { of tumor cells }\end{array}$ \\
\hline
\end{tabular}

Abbreviations: $M A B$, monoclonal antibody; $P A B$, polyclonal antibody; $A R S$, antigen retrieval solution; $R T$, room temperature

oncology) or most recent clinic visit. For overall survival, any cause of death and the most recent clinic visit were used. Relapse was defined as any distant metastasis (liver or other location) or local recurrence. Relapse-free, distant-free, and liver metastases-free survival were censored at last visit to a relevant hospital clinician.

\section{Immunohistochemistry}

$4 \mu \mathrm{m}$ sections of FFPE tissue were cleared at $60^{\circ} \mathrm{C}$ and deparaffinized in xylene. Endogenous peroxidase was blocked by immersion in 0.6\% H2O2 (7210, Merck, Kenilworth, United States of America) in methanol for 15 minutes. Antigen retrieval was performed by cooking slides in a $10 \mathrm{mM}$ citrate $(\mathrm{pH}$ 6) or 10/1 mM Tris/EDTA ( $\mathrm{pH}$ 9) solution for 20 minutes. Nonspecific binding was reduced by with Protein Block Serum Free (X0909, Dako, Santa Clara, United States of America). Antibodies were diluted in Normal Antibody Diluent (Immunologic, Duiven, The Netherlands) and applied on the slides (Table 1). After incubation of post antibody blocking solution for 15 minutes (Immunologic), the secondary antibody PolyHRP-goat anti Mouse/Rabbit IgG (cat. no. VWRKDPVB110HRP, Immunologic) was incubated for 30 minutes. Peroxidase activity was detected by DAB (D5637, Sigma, St. Louis, United States of America) or Bright-DAB (cat. no. VWRKBS04-110, Immunologic) as chromogen 
for 8 minutes. After all incubation steps, except the protein block, slides were washed with PBS-Tween-20 0.1\% four times. Slides were counterstained with hematoxylin and mounted with Pertex (Histolab, Askim, Sweden).

Scoring was performed by at least two independent researchers ( $W M H, W S, L A A B)$, blinded for each other's results and clinical information. Disagreements were resolved by consensus. For ARX and PDX1, negative protein expression in tumor tissue was defined as weak nuclear staining in $<50 \%$ of cells or strong nuclear staining in $<10 \%$ of cells. Positive expression was defined as weak nuclear staining in $>50 \%$ of cells or intermediate/ strong nuclear staining $>10 \%$ of cells (27). For insulin and glucagon, cytoplasmic staining of $>10 \%$ of cells was considered positive expression for the respective peptide hormone. Normal islets, containing a mix of cells expressing ornot expressing the respective peptide hormone were used as positive and negative controls, respectively. If $<10 \%$ of cells had expression, cases were called scattered. DAXX, ATRX, ARID1A was considered negative if $<5 \%$ of cells had positive nuclear staining and if there was non-tumoral tissue present with positive nuclear staining serving as internal control, e.g. islets of Langerhans, stromal cells, endothelial cells or lymphocytes $(26,28)$. All negative cases in the TMA were also stained on whole sections to confirm the results. For H3K36me 3 loss, a cut-off of $30 \%$ of cells was used $(26,29)$. Negative cases without a positive internal control were non-informative. All cytoplasmic staining was ignored.

Ki67 labelling index (LI) was counted in at least 2000 cells by digital image analysis with Sectra (PACS, Sectra AB, Linköping, Sweden), as previously described (30). Digital counts were confirmed by visual assessment. PanNETs were graded by the 2017 WHO criteria (Ki67 G1 <3\%, G2 3 to 20\%, G3 >20\%) (31). If the pathology report also mentioned tumor grade based on Ki67 or mitoses per 10 HPF, the highest grade was used for further analysis as the location of tumor cores not always represents the most proliferative region.

\section{Fluorescence in situ hybridization (FISH)}

After deparaffinization in xylene, $4 \mu \mathrm{m}$ FFPE sections for CDKN2A/CEN9 FISH were pretreated in $0.2 \mathrm{~N} \mathrm{HCL}$ for 20 minutes, cooked in a $10 \mathrm{mM}$ citrate buffer ( $\mathrm{pH} 6)$ for 20 minutes and washed in PBS. Slides were then digested in proteinase $\mathrm{K}$ buffer for 10 minutes at $37^{\circ} \mathrm{C}(5 \mu \mathrm{M}$ Tris- $\mathrm{HCL}, 1 \mu \mathrm{M}$ EDTA, $1 \mu \mathrm{M} \mathrm{NaCl}, 10 \mathrm{mg} / \mathrm{L}$ Proteinase K), washed with PBS and dried. $10 \mu \mathrm{l}$ of CDKN2A/CEN9 probe mix (CDKN2A/CEN 9 Dual Color probe, Zytolight, Bremerhaven, Germany) was applied per slide. Slides were denatured at $78^{\circ} \mathrm{C}$ for 5 
Table 2. Patient and tumor characteristics

\begin{tabular}{|c|c|c|}
\hline & & Insulinomas \\
\hline \multirow[t]{2}{*}{$\overline{\operatorname{Sex}(\%)}$} & Male & $16(46 \%)$ \\
\hline & Female & $19(54 \%)$ \\
\hline Age at surgery & Mean ( $\pm S D)$ & $55( \pm 18)$ \\
\hline Median Follow-up & $\begin{array}{l}\text { Reverse KM + (Mean, IQR } \\
\text { months) }\end{array}$ & $49(57,5-81)$ \\
\hline Median Follow-up & $\begin{array}{l}\text { Observation time; (Mean, IQR } \\
\text { months) }\end{array}$ & $30(52,5-58)$ \\
\hline Relapse & Yes & $3(9 \%)$ \\
\hline (Liver/Other/Local) & No & $32(91 \%)$ \\
\hline \multirow[t]{2}{*}{ Liver Metastases (\%) } & Yes & $3(9 \%)$ \\
\hline & No & $32(91 \%)$ \\
\hline Other distant & Yes & $1(3 \%)$ \\
\hline Metastasis (\%) & No & $34(97 \%)$ \\
\hline Local & Yes & $1(3 \%)$ \\
\hline Recurrence (\%) & No & $34(97 \%)$ \\
\hline Death of all causes $(\%)$ & No & $35(100 \%)$ \\
\hline \multirow[t]{5}{*}{ Location (\%) } & Head & $4(11 \%)$ \\
\hline & Corpus & $4(11 \%)$ \\
\hline & Tail & $18(51 \%)$ \\
\hline & Multifocal & $2(6 \%)$ \\
\hline & Unknown & $7(20 \%)$ \\
\hline Tumor Size $\left(c_{1}\right)$ & $( \pm S D)$ & $1.97( \pm 1.84)$ \\
\hline$\geq 2 \mathrm{~cm}(\%)$ & & $10(29 \%)$ \\
\hline$\geq 3 \mathrm{~cm}(\%)$ & & $3(9 \%)$ \\
\hline \multirow[t]{4}{*}{ Grade (\%) } & 1 & $31(89 \%)$ \\
\hline & 2 & $3(9 \%)$ \\
\hline & 3 & $0(0 \%)$ \\
\hline & Missing & $1(3 \%)$ \\
\hline \multirow[t]{4}{*}{ Resection Margins (\%) } & Free & $17(49 \%)$ \\
\hline & Involved & $7(20 \%)$ \\
\hline & Unsure & $8(23 \%)$ \\
\hline & Not mentioned & $3(9 \%)$ \\
\hline \multirow[t]{3}{*}{ Lymph nodes (\%) } & Free & $14(40 \%)$ \\
\hline & Involved & $0(0 \%)$ \\
\hline & Not Mentioned & $21(60 \%)$ \\
\hline \multirow[t]{2}{*}{$\operatorname{ARX}(\%)$} & Positive & $3(9 \%)$ \\
\hline & Negative & $32(7 \%)$ \\
\hline \multirow[t]{2}{*}{ PDX1 (\%) } & Positive & $34(97 \%)$ \\
\hline & Negative & $1(3 \%)$ \\
\hline \multirow[t]{4}{*}{ ATRX/DAXX (\%) } & ATRX negative & $0(0 \%)$ \\
\hline & DAXX negative & $1(3 \%)$ \\
\hline & Both positive & $33(94 \%)$ \\
\hline & Missing & $1(3 \%)$ \\
\hline \multirow[t]{3}{*}{ Telomeres } & $\begin{array}{l}\text { Alterative Lengthening of } \\
\text { Telomeres }\end{array}$ & $2(6 \%)$ \\
\hline & Normal Telomeres & $31(89 \%)$ \\
\hline & Missing & $2(6 \%)$ \\
\hline \multirow[t]{5}{*}{ CDKN2A } & Normal & $18(51 \%)$ \\
\hline & Monosomal & $1(3 \%)$ \\
\hline & Homozygous loss & $1(3 \%)$ \\
\hline & Not interpretable & $11(31 \%)$ \\
\hline & Not tested & 4 \\
\hline \multirow[t]{3}{*}{ ARID1A and H3K36me3 } & Both positive & $30(97 \%)$ \\
\hline & Missing & $1(3 \%)$ \\
\hline & Not Tested & 4 \\
\hline
\end{tabular}

Abbreviations: $S D$, standard deviation; KM, Kaplan Meier; IQR, interquartile range. 
Continued Table 2. Patient and tumor characteristics

\begin{tabular}{lll}
\hline & & Insulinomas \\
\hline Insulin (\%) & Positive & $28(90 \%)$ \\
& Scattered & $2(7 \%)$ \\
& Negative & $0(0 \%)$ \\
& Missing & $1(3 \%)$ \\
& Not tested & 4 \\
Glucagon (\%) & Positive & $13(42 \%)$ \\
& Scattered & $5(16 \%)$ \\
& Negative & $12(39 \%)$ \\
& Missing & $1(3 \%)$ \\
& Nnot tested & 4 \\
\hline
\end{tabular}

Abbreviations: SD, standard deviation; KM, Kaplan Meier; IQR, interquartile range.

minutes and cooled on ice for 5 minutes. Hybridization was performed in a ThermoBrite (Abbott Laboratories, Chicago, IL) at $37^{\circ} \mathrm{C}$ overnight. After removing coverslips, slides were washed in washing buffer (WB) $1\left(0.4 \times\right.$ SCC, $0.5 \%$ NP- $\left.40,73^{\circ} \mathrm{C}\right)$, WB 2 ( $2 \times$ SCC, $0.1 \%$ NP-40, room temperature), WB 3 ( $2 \times$ SCC, room temperature), and PBS, for 2, 1, 5 minutes, and 20 seconds respectively. Nuclei were counterstained and mounted with Vectashield with DAPI ( $\mathrm{H}-1200$, Vector laboratories, Amsterdam, The Netherlands)

Slides for telomere/centromere FISH were cooked in $10 \mathrm{mM}$ citrate buffer $(\mathrm{pH} 6)$ for 20 minutes, washed in dH2O and dried. Probes (TelC-Cy3 F1002 PNA 180723PL-01, Cent-FITC, F3013 172865, Panagene, Daejeon, Republic of Korea) were diluted in hybridization mix ( $50 \%$ deionized Formamide, 50\% SCC 4x, 5\% Dextran sulphate, Tween-20 0.5\%) at a 400nM concentration and applied on the slides. After 5 minutes denaturation at $84^{\circ} \mathrm{C}$, slides were cooled on ice for 5 minutes before hybridizing at $37^{\circ} \mathrm{C}$ overnight. After removing coverslips, slides were washed in two cycles of $1 \times$ WB (70\% Formamide, 30\% dH2O, 10mM Tris, 15 minutes) and $3 \times$ PBS (2 minutes each time). Nuclei were counterstained with DAPI in PBS 2ug/ml (Sigma-Aldrich, D9542) and coverslips were mounted with Vectashield (H-1000, Vector laboratories). Slides were stored at $4^{\circ} \mathrm{C}$ before imaging and viewed with a Leica DM5500 B using appropriate excitation and emission filters. Images were made at 100x magnification with a Z stack of 14 steps in Leica application Suite X (Leica Microsystems, Rijswijk, The Netherlands).

The numbers of CDKN2A gene probes and centromere probes were counted in at least 50 tumor cells for each case (WMH, WS). At least 9 photographs of tumor tissue (confirmed on H\&E) were made for counting of cells. Only intact non-overlapping nuclei with at least 
one centromere probe were counted. Multiple signals separated $\leq 1$ signal distance were counted as one. If no signals were observed (gene and centromere) in the tumor and surrounding stromal cells or if there was too much background, cases were called non-informative. Homozygous loss was defined as at least $20 \%$ of counted cells lacking CDKN2A probe signals with at least 1 CEP9 probe. Hemizygous deletion of CDKN2A was concluded if $45 \%$ of counted cells had 1 CDKN2A probe and 2 CEP9 probes; monosomy of chromosome 9 (which can be considered hemizygous loss) if $15 \%$ of cells had 1 CDKN2A probe and 1 CEP9 probe. Cut-off values were based on previous literature $(26,32)$. If results were discordant, additional photos were made and at least 100 cells were counted (WMH).

ALT positivity was defined as ultra-bright, intra-nuclear telomere FISH signals, 10x the signal intensity of cumulative single telomere sum intensities in normal stromal/endothelial cells, which are present in more than $1 \%$ of cells $(18,33)$. The percentage of ALT cells $>1 \%$ was determined on 20x magnification by visual assessment ( $\mathrm{WMH})$ in areas of tumor tissue (annotated in H\&E slide), with a cut-off of more than 20 ultra-bright foci per tumor core (max 2000 cells). If less than $1 \%$ by visual assessment, all ultra-bright foci were counted. All ultra-bright foci were confirmed at 100x magnification. Using the same laser/microscope settings, representative ultra-bright foci near stromal cells were photographed and digital greyscale TIFF images exported for each fluorophore channel. Telomere signals were quantified using Telometer (a free custom software ImageJ plug-in, downloaded from (demarzolab.pathology.jhmi.edu/telometer). Rolling ball radius was set at the maximal telomeric signal diameter, which was measured per photograph. Signals were separated by the draw function if necessary. Centromere signals were used as positive hybridization efficiency control and to confirm specific probe binding.

\section{Statistics}

Data was managed and statistical tests were performed in SPSS version 25 (IBM Nederland, Amsterdam, The Netherlands). The Kaplan Meier method was used to plot the effect of variables on events occurring over time, and significance was assessed by the log-rank test. The $\mathrm{X} 2$ or Fishers exact test was used when comparing categorical data between groups. Follow-up time was calculated using a reverse Kaplan Meier for relapse-free survival and observation time was calculated from date of surgery until last visit to a relevant hospital

clinician, irrespective of events. $P$ values $<0.05$ were considered significant. Data was visualized in R version 3.5.1 using packages Beeswarm and Survplot. 


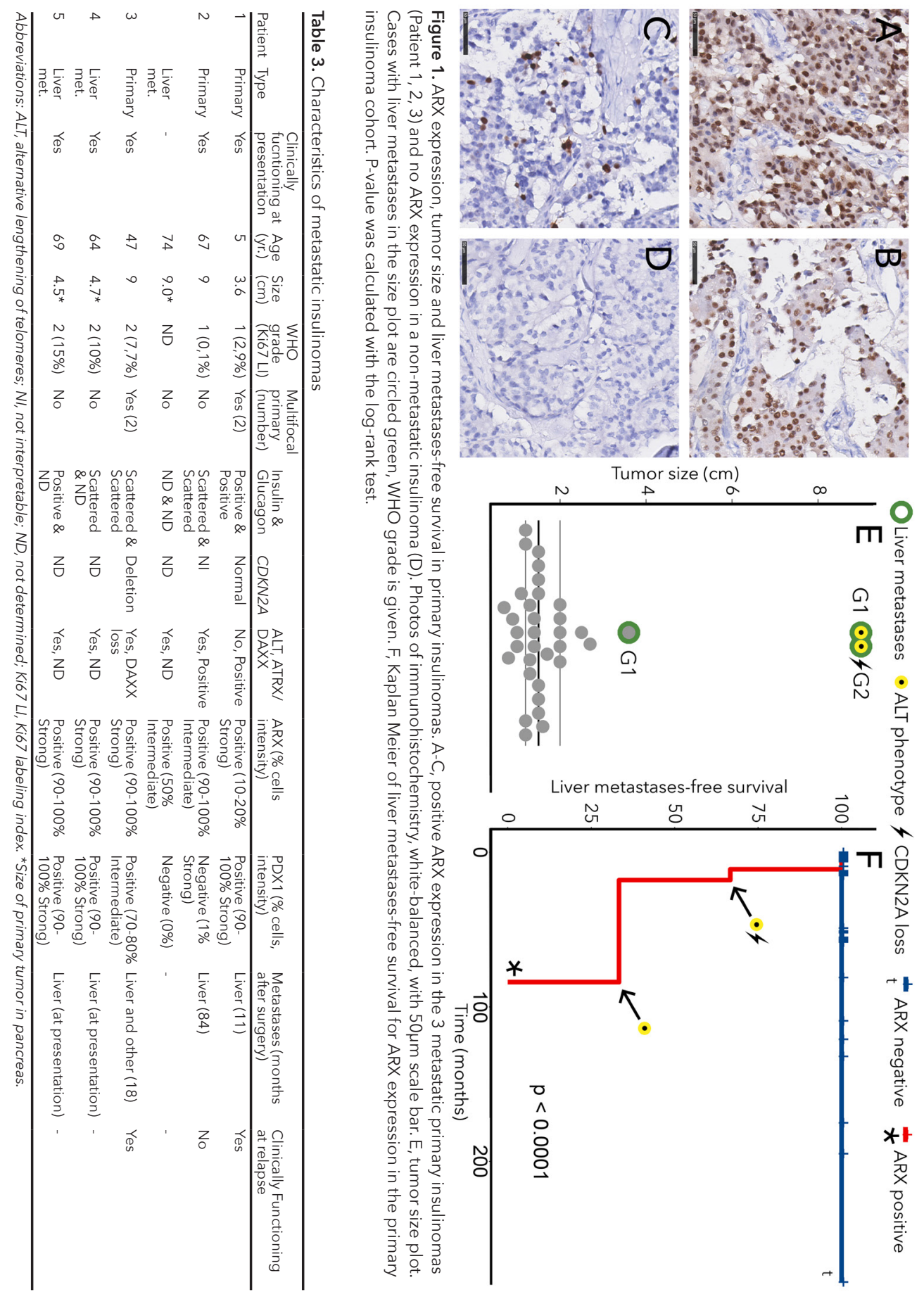




\section{RESULTS}

The cohort consisted of 35 primary insulinomas. The clinical characteristics of the patients are listed in Table 2. All cases were confirmed to be clinically functioning insulinomas by the treating hospital clinician. Three patients developed liver metastases during follow-up, one of whom also had local recurrence and tumor depositions around the uterine appendages. While all metastastic primary cases initially presented with symptomatic hypoglycemia, only two out of three cases had hypoglycemic events at relapse by liver metastasis (Table 3). Overall survival could not be analyzed as there were no deaths during follow-up. The metastatic primary insulinomas were larger than the indolent primary insulinomas, but this did not reach statistical significance (mean 32 indolent insulinomas $1.48 \mathrm{~cm}, \mathrm{SD}$ 0.45, mean 3 metastatic insulinomas $7.20 \mathrm{~cm}$, SD 3.12, t-test p-value $=0.086$; Figure 1). In addition, biopsies of liver metastases of 2 patients with inoperable primary insulinoma were available (Table 3), as well as a biopsy of a corresponding liver metastasis of one of the primary insulinomas in the cohort (Patient 2, Table 3). When the sizes of the primary tumors of the inoperable insulinoma patients were included, the 5 metastatic insulinomas were significantly larger than the indolent cases (mean $1.48 \mathrm{~cm}$, SD 0.45 , metastatic mean $6.16 \mathrm{~cm}, \mathrm{SD} 2.63$, t-test $P=0.016$ ). A size cut-off of $3 \mathrm{~cm}$ perfectly separated all metastatic from indolent insulinomas.

Positive or scattered insulin expression was observed in all insulinomas. Positive or scattered glucagon expression was seen in about $60 \%$ of insulinomas. PDX 1 expression was observed in 34/35 primary insulinomas (97\%), and 2/3 liver metastases; in contrast, only 3/35 (9\%) of primary insulinomas were ARX positive, while all liver metastases were ARX positive (Figure 1, Tables 2 and 3). All ARX positive primary insulinomas developed metastases during follow-up. The ARX positive percentage of cells varied between $10 \%$ and $90 \%$ of cells (Figure 1, Table 3) and PDX1 expression was intermediate to strong in 70-100\% of cells of all positive cases. Two (of 3 ) metastasizing primary insulinomas were multifocal (2 tumors per patient). To exclude the possibility of the smaller tumor being the insulinoma and the larger tumor (tested in the TMA) possibly being an ARX positive non-functional PanNET, whole slides of all multifocal tumors were stained for ARX, PDX1 and insulin. ARX, PDX1, and insulin expression was identical between tumors of the same patient. Thus, strong ARX expression in more than $10 \%$ of cells identified metastatic insulinomas with a $100 \%$ sensitivity and specificity. Of note, one of the metastatic insulinomas showed areas with $<10 \%$ ARX positive cells with scattered positive cells when assessing the whole slide (Patient 
1, Figure 1C). Although no obvious heterogeneity was seen between different tumor cores when scoring for ARX and PDX1, it is conceivable that the number of ARX positive cells was underestimated using TMA cores for other cases. We therefore sought to further confirm our results by reviewing all ARX negative insulinomas by digital image analyses on the TMA similar to the Ki67 count (30). In all these cases expression was observed in far less than $10 \%$ of cells, most often absent or in less than $1 \%$ of cells.

Of 33 insulinomas interpretable by telomere FISH, 2 cases (6\%) had ALT and both developed liver metastases during follow up (Figure 1, Table 3). All tested liver metastasis biopsies had ALT. All insulinomas $(n=34)$ with tumor tissue present in the TMAs had ATRX expression, only one case had heterogenic DAXX loss (also ALT positive). Insulin, glucagon, ARID1A, H3K36me3 IHC and CDKN2A FISH were only tested on 31 cases, as for the last 4 insulinoma cases no unstained TMA slides were available. All cases $(n=30)$ with with tumor tissue present in the TMA had retained H3K36me3 and ARID1A expression. One case with homozygous CDKN2A deletion and one case with monosomal CDKN2A were observed (of 21 interpretable cases; Table 3). The case with homozygous CDKN2A loss developed liver metastases. The metastatic primary insulinomas were grade $1(2 / 3)$ or grade $2(1 / 3)$, both metastasis biopsies were grade 2 (Ki67 labeling index, Table 3).

\section{DISCUSSION}

All metastatic insulinomas in this cohort were larger than $3 \mathrm{~cm}$. Strikingly, all metastatic insulinoma lesions showed ARX expression, which was not observed in any of the indolent primary insulinomas. 4 out of 5 (80\%) metastatic insulinomas had ALT - not reported before in insulinomas - while none of the indolent insulinomas showed ALT. Interestingly, the two recent studies that identified ARX as marker for PanNET relapse after surgery, also included non-metastatic insulinomas: Cejas et al. found no ARX immunohistochemical expression in 17 primary insulinomas (27) and Chan et al. reported ARX mRNA expression (alpha signature) in 1 out of 3 insulinomas. None of the insulinomas in these studies metastasized, but remarkably, the ARX expressing case in the study of Chan et al was also ATRX mutated and very large $(8 \mathrm{~cm})$, while all the negative cases were small $(<2 \mathrm{~cm})$ and ATRX, DAXX, MEN1 wildtype (20). These results are in line with our observation that there is an association between size, ALT and ARX expression in insulinomas. To our knowledge, ALT has not been described before in any insulinoma. Retention of ATRX/DAXX protein expression in one of the insulinomas with ALT may be explained by non-truncating mutations, translocations, or other underlying mutations causing the ALT phenotype (34). In the literature, presence 
of somatic ATRX and DAXX mutations or protein loss detected by immunohistochemistry is uncommon in sporadic insulinoma - in contrast to non-functional PanNET $(12-15,26)$. A recent large whole genome sequencing study definitively established that insulinomas and non-functional PanNETs have distinct genetic underpinnings, and recurrent copy number variations together with ATRX and DAXX mutations are a characteristic feature of non-functional PanNETs (12). However, such alterations might be more prevalent in metastatic insulinomas, of which only few are present in the cohorts previously reported in the literature. Interestingly, in a cohort with multiple rare malignant/metastatic insulinomas, a high number of chromosomal aberrations was strongly associated with metastases (35). As ATRXIDAXX mutations and ALT correlate with copy number variations and chromosomal instability $(21,36)$, this may be a reflection of ATRXIDAXX mutations that were not tested at that time. Loss of the tumor suppressor CDKN2A has been reported once before in a malignant insulinoma (37), and was recently described as a marker of malignant behavior in non-functional PanNET (26).

Several studies have reported large insulinomas to be malignant more often, and the late symptomatology suggests relatively low or acquired insulin production (Figure 2) (1, 38, 39). The ARX transcription factor is not expected to be expressed in insulinomas (40), as it is not expressed in pancreatic beta cells $(41,42)$. In contrast, around $50-60 \%$ of non-functional sporadic PanNETs express ARX (27). Although focal nesidioblastosis was considered as explanation for ARX expression, it was deemed highly unlikely due to the characteristic tumor morphology, random peptide hormone expression, and the presence of metastases (43).

Acquired insulin production could be the result of transdifferentiation of ARX positive non-functional PanNETs or (subclinical) glucagonomas. The latter phenomenon has been shown in mice $(44,45)$, and a recent report described a human non-functional PanNET that progressed into a metastatic insulinoma with liver metastases over the course of 2 years (46). Importantly, all malignant cases in this study had hypoglycemic symptoms at presentation, which resolved after surgery of the primary tumor. All but one metachronous liver metastases (Patient 2) gave new episodes of symptomatic hypoglycemia when diagnosed. Interestingly, in patient 2, both the primary and the corresponding liver metastasis only had few PDX1 positive cells (potential insulin producing cells), so perhaps the solitary liver metastasis $(3 \mathrm{~cm})$ did not grow to a size in which a low percentage of cells could have caused symptoms. 


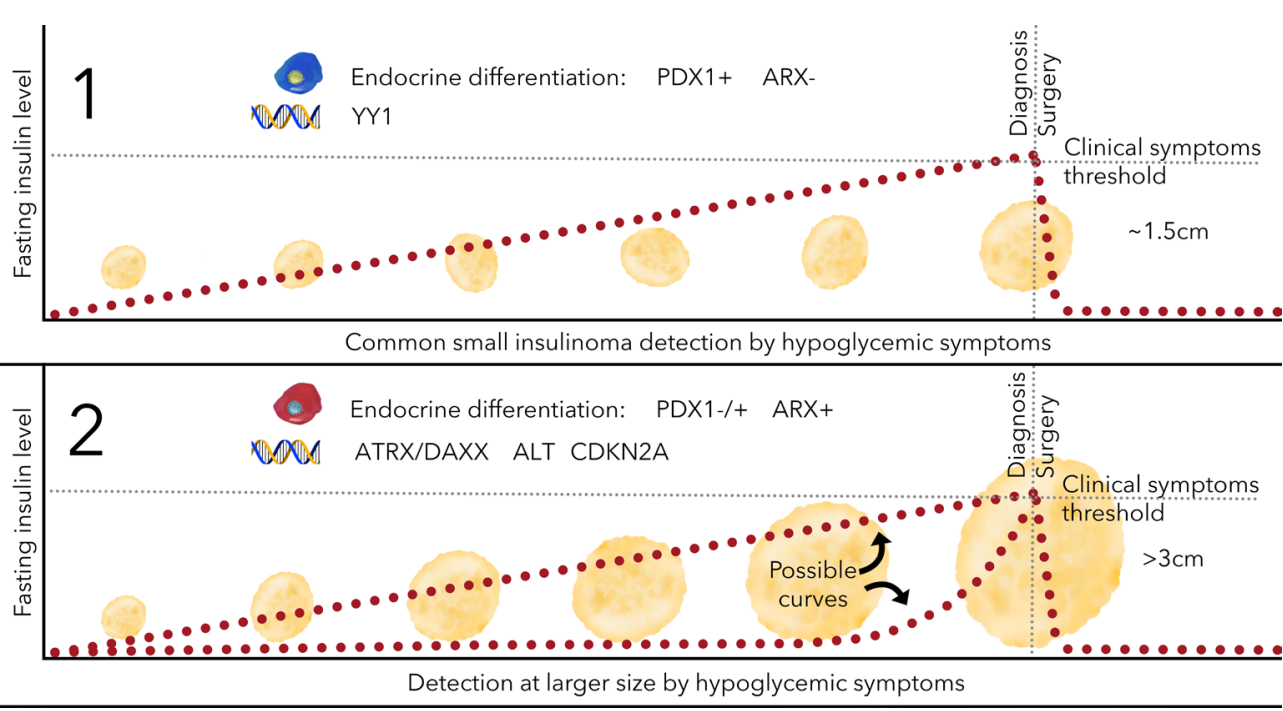

Figure 2. Tumorigenic mechanisms in clinically defined insulinomas. Hypothetic distinct pathways of tumorigenesis in clinical insulinomas based on previously published data in combination with current findings $(1,12,26,35)$. 1, typical small insulinomas characterized by recurrent $Y Y 1$ mutations (25\%), neutral or amplified chromosomal copy numbers, and endocrine transcription factor expression consistent with normal beta-cell differentiation (PDX1+/ ARX-). 2, large insulinomas with distinct tumorigenic mechanisms often seen in non-functional PanNETs and endocrine transcription factor expression inconsistent with normal beta-cell differentiation (ARX+).

There are several strengths of this study. The assay to assess expression of the nuclear transcription factor ARX is very robust. Whole slides that were stained show no variable expression due to fixation, and pancreatic islets, enteroendocrine duodenal/antrum cells serve as positive internal controls. Although several other markers have been proposed based on molecular analyses, protein or mRNA expression, this is the first study to show a clear association of one single immunohistochemical marker with malignant behavior in insulinomas $(35,47,48)$.

The small cohort size and number of metastatic cases is a limitation of this study, caused by the fact that metastatic insulinomas are very rare. Validation of our findings in other cohorts would be of important value. For the limitations inherent to cut-off scoring systems and TMAs, we have tried to minimize their effect by scoring blinded by two independent observers, confirming key percentages by digital analysis, and staining whole slides to confirm immunohistochemical expression when necessary. 
To conclude, large tumor size $(>3 \mathrm{~cm})$ was confirmed to be a strong marker of metastatic behavior in insulinomas. In addition, we found that large metastatic insulinomas are driven by tumorigenic mechanisms often seen in non-functional PanNETs, but not in small indolent insulinomas. Furthermore, we demonstrate that ARX - which is normally not present in betacells - is expressed in a subset of insulinomas and is associated with large tumor size, ALT and metastatic disease. In contrast, ARX expression was absent in any of the small indolent insulinomas in this study or the previous literature. We hypothesize that cellular differentiation and tumorigenic mechanisms more closely related to non-functional PanNETs are important for the development of malignant insulinoma. Our findings with regard to rare metastatic insulinomas may be of value to personalize follow-up and treatment strategies in the future.

\section{CONFLICT OF INTEREST:}

The authors declare that they have no conflict of interest.

\section{ACKNOWLEDGEMENTS}

This work was supported by the Dutch Digestive Foundation/Maag Lever Darm Stichting (grant number CDG 14-020). We thank T. Peeters and A. Meeker for their help with setting up the fluorescence in situ hybridization assay and use of Telometer and P. Cejas for confirming the IHC scoring protocol. 


\section{REFERENCES}

1. Camara-de-Souza AB, Toyoshima MTK, Giannella ML, Freire DS, Camacho CP, Lourenco DM, Jr., et al. Insulinoma: A retrospective study analyzing the differences between benign and malignant tumors. Pancreatology. 2018;18(3):298-303.

2. Nikfarjam M, Warshaw AL, Axelrod L, Deshpande $V$, Thayer SP, Ferrone CR, et al. Improved contemporary surgical management of insulinomas: a 25-year experience at the Massachusetts General Hospital. Ann Surg. 2008;247(1):165-72.

3. Keutgen XM, Schadde E, Pommier RF, Halfdanarson TR, Howe JR, Kebebew E. Metastatic neuroendocrine tumors of the gastrointestinal tract and pancreas: A surgeon's plea to centering attention on the liver. Semin Oncol. 2018;45(4):232-5.

4.

Man D, Wu J, Shen Z, Zhu X. Prognosis of patients with neuroendocrine tumor: a SEER database analysis. Cancer Manag Res. 2018;10:5629-38.

5. Falconi M, Eriksson B, Kaltsas G, Bartsch DK, Capdevila J, Caplin M, et al. ENETS Consensus Guidelines Update for the Management of Patients with Functional Pancreatic Neuroendocrine Tumors and Non-Functional Pancreatic Neuroendocrine Tumors. Neuroendocrinology. 2016;103(2):153-71.

6. Service FJ, McMahon MM, O'Brien PC, Ballard DJ. Functioning insulinoma--incidence, recurrence, and long-term sur vival of patients: a 60-year study. Mayo Clin Proc. 1991;66(7):7119.

7. Kunz PL, Reidy-Lagunes D, Anthony LB, Bertino EM, Brendtro K, Chan JA, et al. Consensus guidelines for the management and treatment of neuroendocrine tumors. Pancreas. 2013;42(4):557-77.

8. Hirshberg B, Cochran C, Skarulis MC, Libutti SK, Alexander HR, Wood BJ, et al. Malignant insulinoma: spectrum of unusual clinical features. Cancer. 2005;104(2):264-72.

9. Dasari A, Shen C, Halperin D, Zhao B, Zhou S, Xu Y, et al. Trends in the Incidence, Prevalence, and Survival Outcomes in Patients With Neuroendocrine Tumors in the United States. JAMA Oncol. 2017;3(10):1335-42.

10. Yao JC, Eisner MP, Leary C, Dagohoy C, Phan A, Rashid A, et al. Population-based study of islet cell carcinoma. Ann Surg Oncol. 2007;14(12):3492-500.

11. Keutgen XM, Nilubol N, Kebebew E. Malignant-func tioning neuroendocrine tumors of the pancreas: A survival analysis. Surgery. 2016;159(5):1382-9.

12. Hong X, Qiao S, Li F, Wang W, Jiang R, Wu H, et al. Whole-genome sequencing reveals distinct genetic bases for insulinomas and non-functional pancreatic neuroendocrine tumours: leading to a new classification system. Gut. 2019.

13. Wang $\mathrm{H}$, Bender A, Wang P, Karakose E, Inabnet WB Libutti SK, et al. Insights into beta cell regeneration for diabetes via integration of molecular landscapes in human insulinomas. Nat Commun. 2017;8(1):767.

14. Cao Y, Gao Z, Li L, Jiang X, Shan A, Cai J, et al. Whole exome sequencing of insulinoma reveals recurrent $T 372 \mathrm{R}$ mutations in YY1. Nat Commun. 2013;4:2810.

15. Lichtenauer UD, Di Dalmazi G, Slater EP, Wieland T, Kuebart A, Schmittfull A, et al. Frequency and clinical correlates of somatic Ying Yang 1 mutations in sporadic insulinomas. J Clin Endocrinol Metab. 2015;100(5):E776-82.
16. Jiao Y, Shi C, Edil BH, de Wilde RF, Klimstra DS,

Maitra A, et al. DAXX/ATRX, MEN1, and mTOR pathway genes are frequently altered in pancreatic neuroendocrine tumors. Science. 2011;331(6021):1199-203.

17. Scarpa A, Chang DK, Nones K, Corbo V, Patch A-M, Bailey $P$, et al. Whole-genome landscape of pancreatic neuroendocrine tumours. Nature. 2017;543(7643):65-71.

18. Heaphy CM, de Wilde RF, Jiao Y, Klein AP, Edil BH, Shi $C$, et al. Altered telomeres in tumors with ATRX and DAXX mutations. Science. 2011;333(6041):425.

19. Hechtman JF, Klimstra DS, Nanjangud G, Frosina D, Shia J, Jungbluth AA. Performance of DAXX Immunohistochemistry as a Screen for DAXX Mutations in Pancreatic Neuroendocrine Tumors. Pancreas. 2019;48(3):396-9.

20. Chan CS, Laddha SV, Lewis PW, Koletsky MS, Robzyk

K, Da Silva E, et al. ATRX, DAXX or MEN1 mutant pancreatic

neuroendocrine tumors are a distinct alpha-cell signature subgroup. Nat Commun. 2018;9(1):4158.

21. Marinoni I, Kurrer AS, Vassella E, Dettmer M, Rudolph $T$, Banz $V$, et al. Loss of DAXX and ATRX are associated with chromosome instability and reduced survival of patients with pancreatic neuroendocrine tumors. Gastroenterology. 2014;146(2):453-60 e5.

22. Singhi AD, Liu TC, Roncaioli JL, Cao D, Zeh HJ,

Zureikat $A H$, et al. Alternative Lengthening of Telomeres and Loss of DAXX/ATRX Expression Predicts Metastatic Disease and Poor Survival in Patients with Pancreatic Neuroendocrine Tumors. Clin Cancer Res. 2017;23(2):600-9.

23. Kim JY, Brosnan-Cashman JA, An S, Kim SJ, Song

KB, Kim MS, et al. Alternative Lengthening of Telomeres in Primary Pancreatic Neuroendocrine Tumors Is Associated with Aggressive Clinical Behavior and Poor Survival. Clin Cancer Res. 2017;23(6):1598-606.

24. Chou A, Itchins M, de Reuver PR, Arena J, Clarkson A, Sheen A, et al. ATRX loss is an independent predictor of poor survival in pancreatic neuroendocrine tumors. Hum Pathol. 2018;82:249-57

25. Park JK, Paik WH, Lee K, Ryu JK, Lee SH, Kim YT. DAXX/ATRX and MEN1 genes are strong prognostic markers in pancreatic neuroendocrine tumors. Oncotarget. 2017;8(30):49796-806.

26. Roy S, LaFramboise WA, Liu T-C, Cao D, Luvison A Miller C, et al. Loss of Chromatin-Remodeling Proteins and/or CDKN2A Associates With Metastasis of Pancreatic Neuroendocrine Tumors and Reduced Patient Survival Times. Gastroenterology. 2018;154(8):2060-3.e8.

27. Cejas P, Drier Y, Dreijerink KMA, Brosens LAA, Deshpande $V$, Epstein $C B$, et al. Enhancer signatures stratify and predict outcomes of non-functional pancreatic neuroendocrine tumors. Nat Med. 2019;25(8):1260-5.

28. de Wilde RF, Heaphy CM, Maitra A, Meeker AK, Edil $\mathrm{BH}$, Wolfgang $\mathrm{CL}$, et al. Loss of ATRX or DAXX expression and concomitant acquisition of the alternative lengthening of telomeres phenotype are late events in a small subset of MEN-1 syndrome pancreatic neuroendocrine tumors. Mod Pathol. 2012;25(7):10339. 


\section{Chapter 7}

29

Ho TH, Park IY, Zhao H, Tong P, Champion MD, Yan $\mathrm{H}$, et al. High-resolution profiling of histone h3 lysine 36 trimethylation in metastatic renal cell carcinoma. Oncogene. 2016;35(12):1565-74.

30. Conemans EB, Brosens LAA, Raicu-Ionita GM, Pieterman CRC, de Herder WW, Dekkers OM, et al. Prognostic value of WHO grade in pancreatic neuro-endocrine tumors in Multiple Endocrine Neoplasia type 1: Results from the DutchMEN1 Study Group. Pancreatology. 2017;17(5):766-72.

31. Inzani F, Petrone G, Rindi G. The New World Health Organization Classification for Pancreatic Neuroendocrine Neoplasia. Endocrinol Metab Clin North Am. 2018;47(3):463-70.

32. Chung CT, Santos Gda C, Hwang DM, Ludkovski O, Pintilie M, Squire JA, et al. FISH assay development for the detection of $\mathrm{p} 16 / \mathrm{CDKN} 2 \mathrm{~A}$ deletion in malignant pleural mesothelioma. J Clin Pathol. 2010;63(7):630-4.

33 Hackeng WM, Morsink FHM, Moons LMG, Heaphy CM, Offerhaus GJA, Dreijerink KMA, et al. Assessment of ARX expression, a novel biomarker for metastatic risk in pancreatic neuroendocrine tumors, in endoscopic ultrasound fine-needle aspiration. Diagn Cytopathol. 2019:Accepted for publication. 34. Brosnan-Cashman JA, Graham MK, Heaphy CM. Genetic alterations associated with ALTered telomeres. Oncotarget. 2018;9(73):33739-40.

35. Jonkers YM, Claessen SM, Perren A, Schmid S, Komminoth $P$, Verhofstad AA, et al. Chromosomal instability predicts metastatic disease in patients with insulinomas. Endocr Relat Cancer. 2005;12(2):435-47.

36. Pea A, Yu J, Marchionni L, Noe M, Luchini C, Pulvirenti A, et al. Genetic Analysis of Small Well-differentiated Pancreatic Neuroendocrine Tumors Identifies Subgroups With Differing Risks of Liver Metastases. Ann Surg. 2020;271(3):566-73.

37. Bartsch DK, Kersting M, Wild A, Ramaswamy A Gerdes B, Schuermann M, et al. Low frequency of p16(INK4a) alterations in insulinomas. Digestion. 2000;62(2-3):171-7.

38. Ueda K, Taira T, Hakoda H, Nakata S, Okata S, Nagai

$T$, et al. Giant insulinoma: report of a case and review of published reports. Surg Case Rep. 2016;2(1):136.

39. Crippa S, Zerbi A, Boninsegna L, Capitanio V, Partelli S, Balzano G, et al. Surgical management of insulinomas: short- and long-term outcomes after enucleations and pancreatic resections. Arch Surg. 2012;147(3):261-6.

40. Wang Z, You J, Xu S, Hua Z, Zhang W, Deng T, et al. Colocalization of insulin and glucagon in insulinoma cells and developing pancreatic endocrine cells. Biochem Biophys Res Commun. 2015;461(4):598-604

41. Baron M, Veres A, Wolock SL, Faust AL, Gaujoux R Vetere $A$, et al. A Single-Cell Transcriptomic Map of the Human and Mouse Pancreas Reveals Inter- and Intra-cell Population Structure. Cell Syst. 2016;3(4):346-60 e4.

42. Muraro MJ, Dharmadhikari G, Grun D, Groen N, Dielen T, Jansen E, et al. A Single-Cell Transcriptome Atlas of the Human Pancreas. Cell Syst. 2016;3(4):385-94 e3.

43. Bhatti TR, Ganapathy K, Huppmann AR, Conlin L, Boodhansingh KE, MacMullen C, et al. Histologic and Molecular Profile of Pediatric Insulinomas: Evidence of a Paternal Parent-of-Origin Effect. J Clin Endocrinol Metab. 2016;101(3):91422.

44. Bertolino $\mathrm{P}$, Tong WM, Herrera PL, Casse $\mathrm{H}$, Zhang CX, Wang ZQ. Pancreatic beta-cell-specific ablation of the multiple endocrine neoplasia type 1 (MEN1) gene causes full penetrance of insulinoma development in mice. Cancer Res. 2003;63(16):4836-41.

45. Lu J, Herrera PL, Carreira C, Bonnavion R, Seigne C, Calender A, et al. Alpha cell-specific Men1 ablation triggers the transdifferentiation of glucagon-expressing cells and insulinoma development. Gastroenterology. 2010;138(5):1954-65.

46. Arslan MS, Ozbek M, Karakose M, Tutal E, Ucan B, Yilmazer D, et al. Transformation of nonfunctioning pancreatic tu mor into malignant insulinoma after 3 years: an uncommon clinical course of insulinoma. Arch Endocrinol Metab. 2015;59(3):270-2. 47. Alkatout I, Friemel J, Sitek B, Anlauf M, Eisenach PA Stuhler K, et al. Novel prognostic markers revealed by a proteomic approach separating benign from malignant insulinomas. Mod Pathol. 2015;28(1):69-79.

48. Murat Cde B, da Rosa PW, Fortes MA, Correa L, Machado MC, Novak EM, et al. Differential expression of genes encoding proteins of the HGF/MET system in insulinomas. Diabetol Metab Syndr. 2015; 7:84. 


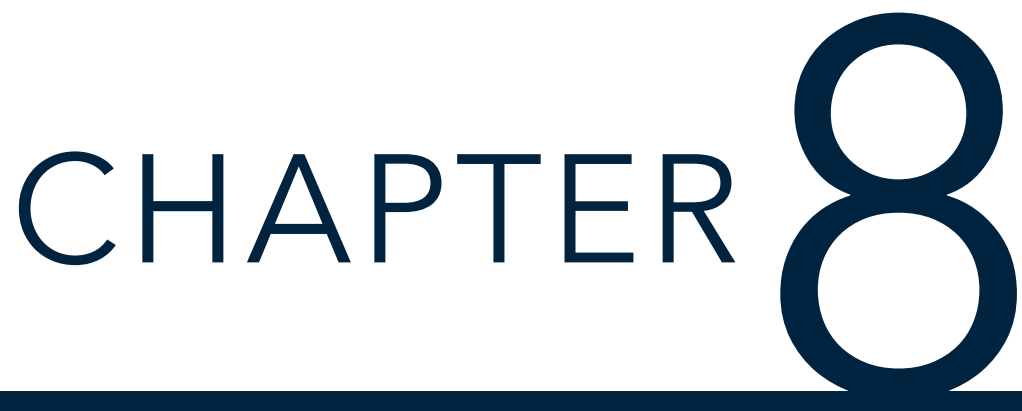

Non-Functional Pancreatic Neuroendocrine Tumors: ATRX/DAXX and Alternative Lengthening of Telomeres (ALT) Assess Prognosis Independently from Islet-Cell Subtype and Tumor Size

Wenzel M. Hackeng, Lodewijk A.A. Brosens, Joo Young Kim, Roderick J. O’Sullivan, You-Na Sung, Ta-Chiang Liu, Dengfeng Cao, Michelle Heayn, Jacqueline Brosnan-Cashman, Soyeon An, Folkert H.M. Morsink, Charlotte M. Heidsma, Gerlof D. Valk, Menno R. Vriens, Els J.M. Nieveen van Dijkum, G. Johan A. Offerhaus, Koen M.A. Dreijerink, Herbert J. Zeh, Amer H. Zureikat, Melissa E. Hogg, Kenneth Lee, David Geller, J. Wallis Marsh, Alessandro Paniccia, Melanie C. Ongchin, James F. Pingpank, Nathan Bahary, Muaz Aijazi, Randall E. Brand, Jennifer S. Chennat, Rohit Das, Kenneth E. Fasanella, Asif Khalid, Kevin McGrath, Savreet Sarkaria, Harkirat Singh, Adam Slivka, Michael A. Nalesnik, Xiaoli Han, Marina N. Nikiforova, Rita T. Lawlor, Andrea Mafficini, Borislav Rusev, Vincenzo Corbo, Claudio Luchini, Samantha Bersani, Antonio Pea, Sara Cingarlini, Luca Landoni, Roberto Salvia, Massimo Milione, Michele Milella, Aldo Scarpa, Seung-Mo Hong, Christopher M. Heaphy, Aatur D. Singhi 


\section{ABSTRACT}

Recent studies have found ARX/PDX1, ATRX/DAXX, and alternative lengthening of telomeres (ALT) to be promising prognostic biomarkers for non-functional pancreatic neuroendocrine tumors (NF-PanNETs). However, they have not been comprehensively evaluated, especially among small NF-PanNETs $(\leq 2.0 \mathrm{~cm}$ ). Moreover, their status in neuroendocrine tumors (NETs) from other sites remains unknown.

An international cohort of 1,322 NETs was evaluated by immunolabeling for ARX/ PDX1 and ATRX/DAXX, and telomere-specific fluorescence in situ hybridization for ALT. This cohort included 561 primary NF-PanNETs, 107 NF-PanNET metastases, and 654 primary, non-pancreatic non-functional NETs and NET metastases. The results were correlated with numerous clinicopathologic features including relapse-free survival (RFS).

ATRX/DAXX loss and ALT were associated with several adverse prognostic findings and distant metastasis/recurrence $(p<0.001)$. The 5 -year RFS rates for patients with ATRX/DAXX-negative and ALT-positive NF-PanNETs were $40 \%$ and $42 \%$ as compared to $85 \%$ and $86 \%$ for wild-type NF-PanNETs $(p<0.001$ and $p<0.001)$. Shorter 5 -year RFS rates for $\leq 2.0 \mathrm{~cm}$ NF-PanNETs patients were also seen with ATRX/DAXX loss (65\% vs. 92\%, $p=0.003$ ) and ALT (60\% vs. 93\%, $p<0.001)$. By multivariate analysis, ATRX/DAXX and ALT status were independent prognostic factors for RFS. Conversely, classifying NF-PanNETs by ARX/PDX1 expression did not independently correlate with RFS. Except for $4 \%$ of pulmonary carcinoids, ATRX/DAXX loss and ALT were only identified in primary (25\% and $29 \%$ ) and NF-PanNET metastases ( $62 \%$ and $71 \%$ ).

ATRX/DAXX and ALT should be considered in the prognostic evaluation of NFPanNETs including $\leq 2.0 \mathrm{~cm}$ tumors, and are highly specific for pancreatic origin among NET metastases of unknown primary. 


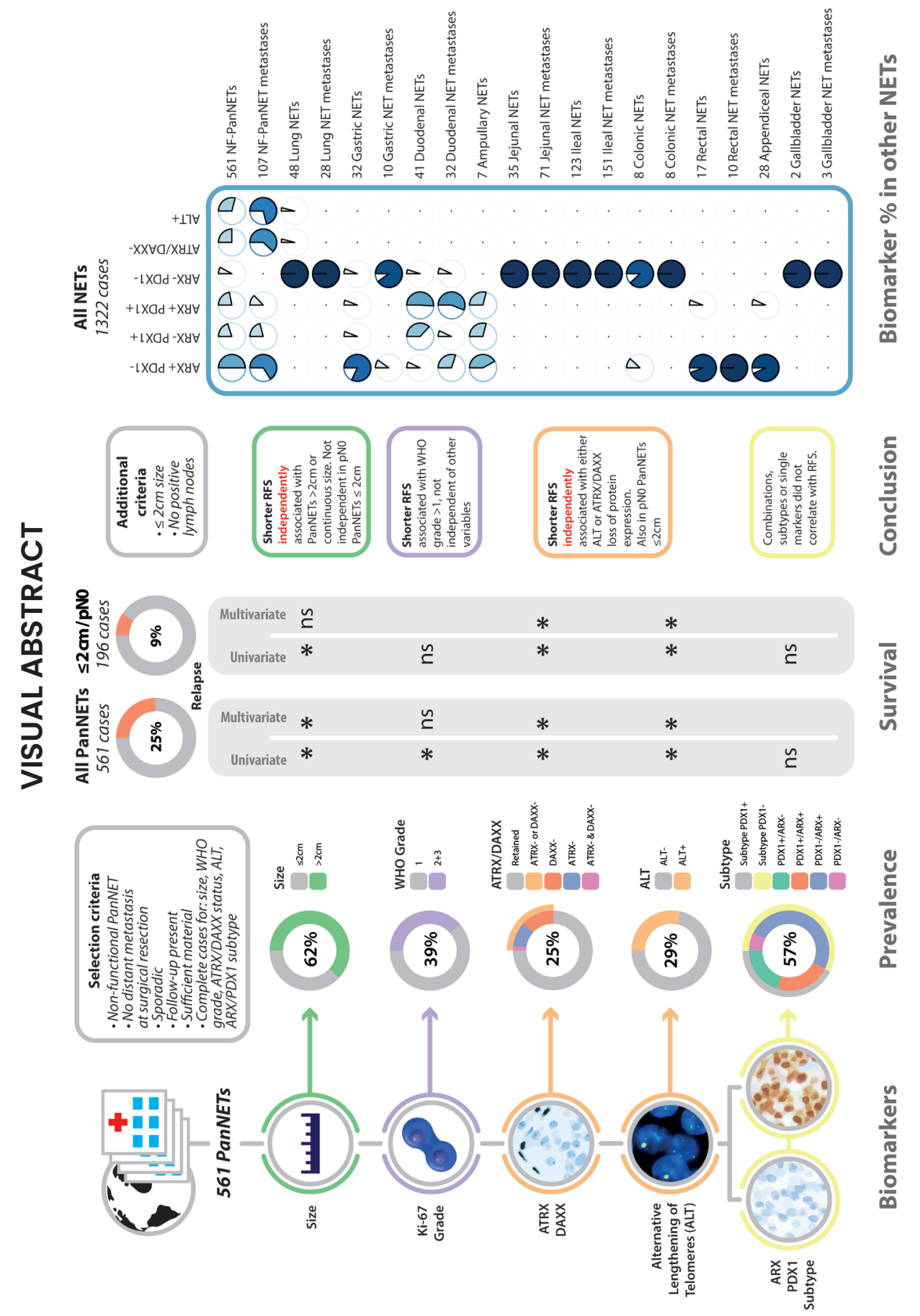




\section{WHAT IS ALREADY KNOWN ABOUT THIS SUBJECT?}

- The frequent detection of non-functional pancreatic neuroendocrine tumors (NFPanNETs), especially those measuring $\leq 2.0 \mathrm{~cm}$, represents a treatment dilemma.

- While most NF-PanNETs are clinically indolent, a subset may behave aggressively and metastasize widely.

- Advancements in molecular techniques have identified several prognostic biomarkers for PanNETs and include the status of ARX, PDX1, ATRX, DAXX, and alternative lengthening of telomeres (ALT).

- The evaluation of these putative biomarkers has largely been restricted to singlecenter studies, limited in study cohort size and assessed in diverse patient populations, such as those with functional PanNETs, synchronous distant metastases, and germline predisposition syndromes.

- The prognostic utility of ARX, PDX1, ATRX, DAXX, and ALT in small NF-PanNETs $(\leq 2.0 \mathrm{~cm})$ and their status in neuroendocrine tumors (NETs) from other sites are unknown.

\section{WHAT ARE THE NEW FINDINGS?}

- Within a multi-institutional, international cohort of NF-PanNETs, loss of ATRX/DAXX and the presence of ALT correlated with several adverse prognostic pathologic features and distant metastases/recurrence upon follow-up.

- Relapse-free survival (RFS) rates were shorter for patients, and, in particular, for those patients with small NF-PanNETs $(\leq 2.0 \mathrm{~cm}$ ) with ATRX/DAXX loss and/or ALT positivity.

- The status of ATRX/DAXX and ALT were independent, negative prognostic biomarkers for NF-PanNETs.

- Classifying NF-PanNETs by ARX/PDX1 expression did not independently correlate with RFS.

-In addition to primary NF-PanNETs and NF-PanNET metastases, only a small subset of pulmonary carcinoids exhibited ATRX/DAXX loss and ALT.

\section{HOW MIGHT IT IMPACT ON CLINICAL PRACTICE IN THE FORESEEABLE FUTURE?}

- Incorporating the status of ATRX/DAXX by immunohistochemistry and ALT by telomere-specific fluorescence in situ hybridization as prognostic biomarkers for NFPanNETs is clinically feasible and inexpensive.

- In the setting of a NET metastasis of unknown origin, ATRX/DAXX and ALT are highly specific diagnostic biomarkers to indicate the possibility of a pancreatic primary. 


\section{INTRODUCTION}

Pancreatic neuroendocrine tumors (PanNETs) are the 2nd most common malignancy of the pancreas and comprise a heterogeneous group of neoplasms. Historically, the incidence of PanNETs has been low, and patients often presented with hormonal hypersecretion (functional PanNETs). However, with increasing use of abdominal imaging, the incidence of PanNETs, especially non-functional PanNETs (NF-PanNETs), has risen rapidly (1). The 5 -year survival of patients with NF-PanNETs is reported to be as low as $54 \%$ and is highly dependent on the presence of distant metastases (2). Patients with localized disease have a reported 5 -year survival of $93 \%$ as compared to $27 \%$ for patients with metastatic disease. Many patients develop infiltrative and widely metastatic neoplasms, while others may present with slow growing, indolent tumors. In fact, in recent years, the overtreatment of NFPanNETs has been a subject of debate and an observational approach may be warranted for a subset of patients $(3,4)$.

Routinely used prognostic biomarkers for NF-PanNETs are tumor size, grade, and stage. Current recommendations by the National Comprehensive Cancer Network, European Neuroendocrine Tumor Society and North American Neuroendocrine Tumor Society are to surgically resect NF-PanNETs $>2.0 \mathrm{~cm}$ in size with negative margins and regional lymphadenectomy due to their association with metastatic spread (5-7). A limited surgical procedure, or even surveillance, can be considered in NF-PanNETs $\leq 2.0 \mathrm{~cm}$ as these neoplasms often do not metastasize. Nevertheless, NF-PanNETs $\leq 2.0 \mathrm{~cm}$ can be aggressive, including those initially classified as clinically indolent (8-11). In addition to tumor size, the World Health Organization (WHO) advocates grading of NF-PanNETs based on proliferative activity that includes mitotic index and Ki-67 immunohistochemistry (12). Many studies suggest tumors of at least WHO grade 2 are associated with a worse clinical behavior (13). However, both mitotic index and Ki-67 measurements are susceptible to sampling issues, interpretation errors, and may not truly reflect the clinical behavior of these neoplasms as WHO grade 1 tumors can also develop distant metastases $(14,15)$. Thus, additional biomarkers are needed to improve the prognostic classification and management of patients.

Advancements in molecular technologies have identified several putative prognostic biomarkers for NF-PanNETs. Whole exome and whole genome sequencing studies have found recurrent alterations in alpha-thalassemia/mental retardation X-linked chromatin 
remodeler $(A T R X)$ and death domain-associated protein (DAXX) that are associated with metastatic disease (16-20). Mutations in these genes often result in loss of nuclear expression of their respective proteins by immunohistochemistry. Furthermore, ATRX/ DAXX loss frequently coincides with the presence of alternative lengthening of telomeres $(\mathrm{ALT})$, a telomerase-independent telomere maintenance mechanism, which can reliably be assayed using telomere-specific fluorescence in situ hybridization (FISH) (21). Loss of ATRX/DAXX and the presence of ALT correlate with a shorter relapse-free survival (RFS) (2224). Additionally, whole transcriptome and epigenome studies have found the differential expression of the transcription factors, aristaless-related homeobox gene (ARX) and pancreatic and duodenal homeobox 1 (PDX1), can also determine the risk of metastatic disease $(25,26)$. PDX1 expression is typically associated with an indolent clinical behavior, while the expression of ARX or the lack of both proteins correlates with an aggressive disease course. The status of these transcription factors in PanNETs was recently reported to be a prognostic biomarker for RFS and independent of tumor size, WHO grade, and ALT (26).

However, the prognostic significance of ARX and PDX1 in NF-PanNETs was assessed in a study cohort limited in number and enriched towards patients with germline predisposition syndromes (26). In contrast, loss of ATRX/DAXX and the presence of ALT in NF-PanNETs have been evaluated by multiple investigators, but their clinical utility amongst patients with NF-PanNETs of $\leq 2.0 \mathrm{~cm}$ has not been comprehensively analyzed. Moreover, the status of ARX, PDX1, ATRX/DAXX, and ALT in NF-PanNET metastases and neuroendocrine tumors (NETs) from other organ sites remains largely unexplored and may be of clinical and prognostic relevance within these neoplasms. In fact, in addition to the pancreas, the expression of ARX and PDX1 are key factors for endocrine progenitor cells of other organ sites (27). Therefore, the objectives of this study were to [1] determine the correlation of ARX and PDX1 expression with clinicopathologic features in a large, international, multi-institutional cohort of sporadic NF-PanNETs without distant metastatic disease at presentation, [2] validate the prognostic significance of these transcription factors, ATRX/ DAXX, and ALT in NF-PanNETs and include a separate analysis among tumors of $\leq 2.0 \mathrm{~cm}$, and [3] comprehensively evaluate the status of all 5 biomarkers in NF-PanNETs metastases and NETs from other organ sites. 


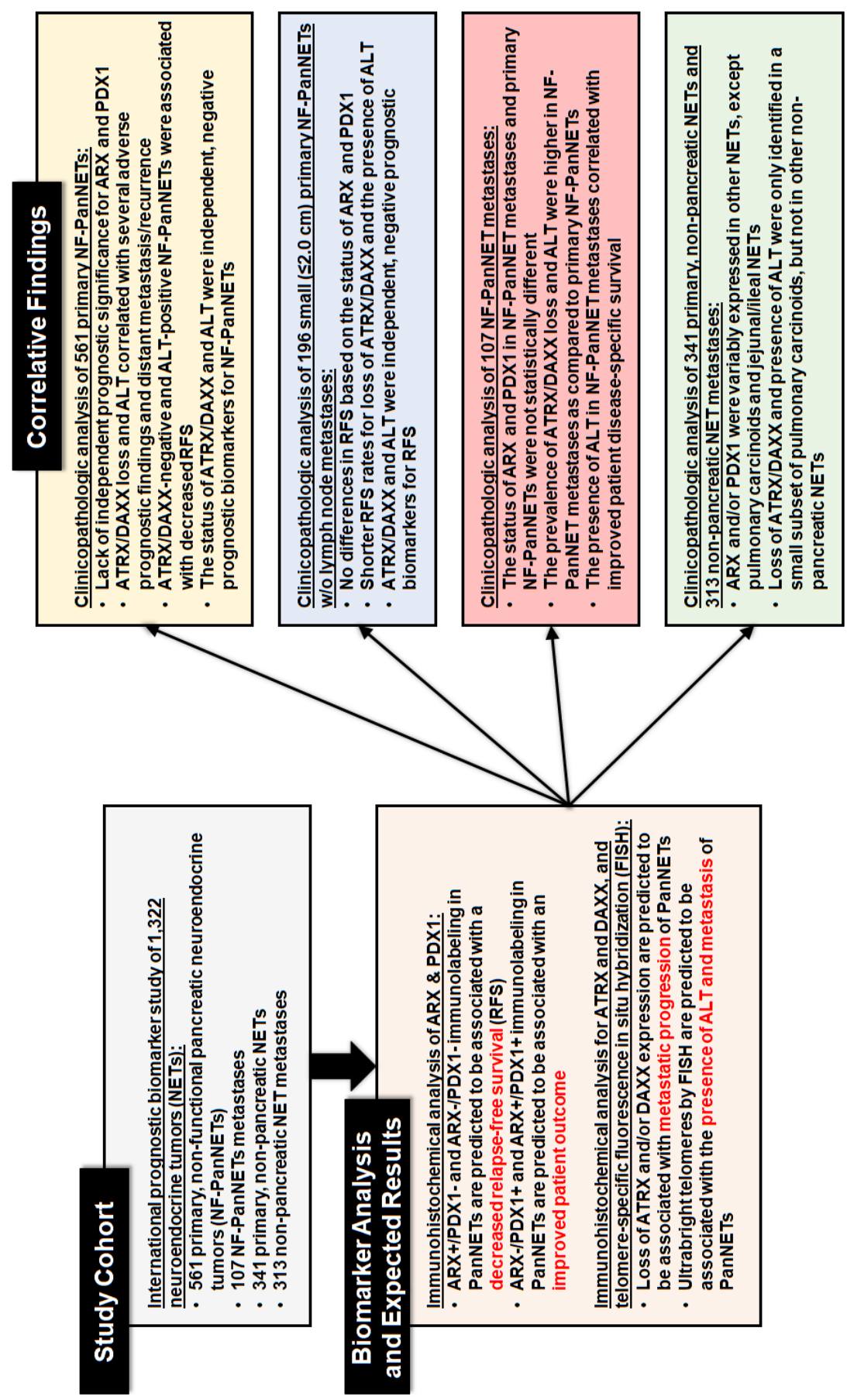

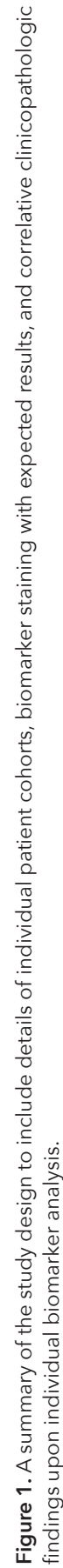




\section{MATERIALS AND METHODS}

\section{Study design and case selection}

The study design is summarized in Figure 1 and includes the overall patient cohort, biomarker staining and expected results, and correlative clinicopathologic findings. Study approval was obtained from the University Medical Center (UMC) Utrecht Biobank Research Ethics Committee, Verona University and Hospital Trust Ethical Committee, Asan Medical Center, and Institutional Review Boards from Washington University and University of Pittsburgh (IRB\# PRO13020493). The surgical pathology archives from Amsterdam UMC, UMC Utrecht, ARC-Net Research Center, Asan Medical Center, Barnes-Jewish Hospital and University of Pittsburgh Medical Center (UPMC) were queried for neuroendocrine neoplasms of the pancreas between 1991 and 2017 that underwent enucleation, central pancreatectomy, pancreaticoduodenectomy or distal pancreatectomy. Cases were crossreferenced with clinical and follow-up data obtained from patient paper and/or electronic medical records. The study inclusion criteria consisted of the following: a solitary, clinically non-functional, well-differentiated neuroendocrine tumor (confirmed with positive immunolabeling for neuroendocrine markers: synaptophysin and/or chromogranin A) centered within the pancreas; absence of a confirmed or suspected genetic syndrome associated with pancreatic neuroendocrine neoplasms: multiple endocrine neoplasia type 1 (MEN1) syndrome, von Hippel-Lindau (VHL) syndrome, neurofibromatosis type 1 (NF1) syndrome, familial atypical multiple mole melanoma (FAMMM) syndrome and tuberous sclerosis complex (TSC) syndrome; surveillance and survival data; and cases with sufficient material for ancillary studies. Patients with a confirmed or suspected genetic syndrome associated with PanNETs were excluded considering these patients may develop multiple primary PanNETs, and, within these scenarios, sampling of each primary from the same patient is impractical for prognostication purposes. It is important to underscore that exclusion of patients was based on both clinical and germline data; however, formal germline testing for all patients included within this study was not performed.

In total, 561 patients with a resected NF-PanNET fulfilled the aforementioned criteria and consisted of the following: 28 patients from Amsterdam UMC, 27 patients from UMC Utrecht, and 82 patients from ARC-Net Research Center (Supplementary Table 1), 168 patients from Asan Medical Center (Supplementary Table 2); and 79 patients from BarnesJewish Hospital and 177 patients from UPMC (Supplementary Table 3). Within the same timeframe, the surgical pathology archives at Barnes-Jewish Hospital and UPMC were 
also queried to identify NF-PanNET distant organ metastases and yielded 107 patients with sufficient pathologic material available of their metastatic specimen for ancillary studies. Further, NETs from other organ sites to include primaries $(n=341)$ and distant organ metastases $(n=313)$ were identified within the UPMC surgical pathology archives for subsequent analysis. Archival pathologic material from all 1,322 neoplasms was used to create high-density tissue microarrays (TMAs) as previously described (18, 23, 24, 28, 29). While the TMAs were used for telomere-specific fluorescence in situ hybridization (FISH), whole tumor sections for 256 primary NF-PanNETs, 63 NF-PanNET metastases and 132 primary, non-pancreatic NETs, and 62 non-pancreatic NET metastases were evaluated immunohistochemically for ARX, PDX1, ATRX and DAXX.

Clinical and demographic data were reviewed for each case. Corresponding pathology gross reports and hematoxylin-and-eosin (H\&E) stained slides were also reviewed for the following pathologic features: tumor size, location, lymphovascular invasion, perineural invasion, extension outside of the pancreas and regional lymph node metastasis. Each primary NF-PanNET was graded using the 2017/2019 World Health Organization (WHO) classification system for pancreatic neuroendocrine neoplasms on the basis of mitotic rate and Ki-67 immunohistochemistry as Grade 1 (G1), <2 mitoses/2 mm2 and Ki-67 of $<3 \%$; Grade 2 (G2), 2 to 20 mitoses/2 mm2 or Ki-67 of 3\% to 20\%; or Grade 3 (G3), > 20 mitoses/2 mm2 or Ki-67 of $>20 \%$. $(12,30)$ For cases with discordant mitotic rate and $\mathrm{Ki}-67$ measurements, the highest grade was assigned. Pathologic primary tumor classification was determined according to the 8th edition of the American Joint Committee on Cancer (AJCC) Staging Manual, which is based on tumor size and extent of invasion (31). Followup information was extracted from the patient's medical records to include data on surveillance, relapse-free survival (RFS), and disease-specific survival (DSS). Pancreatic and non-pancreatic neuroendocrine carcinomas were excluded from this study.

\section{Immunohistochemistry}

TMAs or whole section tumor slides were deparaffinized with serial xylene treatments and subjected to antigen retrieval using heated citrate solution. Immunolabeling for ARX (MABN102 mouse monoclonal, Millipore), PDX1 (AB134150 rabbit monoclonal, Abcam), ATRX (HPA001906 rabbit polyclonal, Sigma Aldrich), and DAXX (HPA008736 rabbit polyclonal, Sigma Aldrich or AB32140 rabbit monoclonal, Abcam) were performed either on an automated Ventana Benchmark XT system using the biotin-free Ventana OptiView 
DAB IHC Detection Kit (Ventana Medical Systems) or manually (Supplementary Materials and Methods).

Assessment of ARX, PDX1, ATRX, and DAXX was done blinded to any patient data including outcome. For ARX and PDX1, "positive" expression was defined as nuclear staining of $>10 \%$ of neoplastic cells as previously described $(28,32)$. Tumor tissue with $\leq 10 \%$ of nuclear expression was scored as "negative" expression. Surrounding islets within the uninvolved pancreas were used as a positive internal control. Immunolabeling for ATRX and DAXX was considered "positive" or preserved if $>5 \%$ of neoplastic cells had nuclear staining, as previously described $(22,23)$. Neoplasms were scored as "negative" or loss of staining for ATRX or DAXX if the pattern was that of cytoplasmic accumulation with nuclear clearing, as long as adequate internal controls (e.g., nuclear labeling of adjacent endothelial cells, lymphocytes and/or islets of Langerhans) were present. Intratumoral heterogeneity or heterogeneous staining of ATRX and DAXX was defined as the clear presence of two distinct populations of tumor cells demonstrating preserved and loss of nuclear staining (24). For subsequent statistical analysis, cases with heterogeneous staining were scored as loss or negative staining.

\section{Fluorescence in situ hybridization}

Telomere-specific FISH was performed as previously described using a fluorescently tagged telomeric-C PNA probe for each TMA (Supplementary Materials and Methods) (33). Scoring for ALT was performed by assessing at least 250 nuclei from all for each case. Using previously described criteria, ALT-positive cases were defined by the presence of large, ultrabright intranuclear foci consistent with telomere FISH signals in at least $1 \%$ of tumor nuclei and the total signal intensity for individual foci $>10$ fold than telomere signals from stromal cells. $(23,24)$ Importantly, areas of necrosis were excluded from evaluation.

\section{Statistical analysis}

Chi-squared analysis or Fisher exact tests were used to compare categorical data, and MannWhitney $U$ test was used to compare continuous variables. Survival curves were constructed using the Kaplan-Meier method and differences between groups were evaluated by the log-rank test. Relapse-free survival (RFS) was calculated from the date of surgery to the date of first distant metastasis/local recurrence after surgery and censored at the date of last follow-up or death. The prognostic significance of clinical and pathologic characteristics was determined using univariate Cox regression analysis. Multivariate analyses of significant 


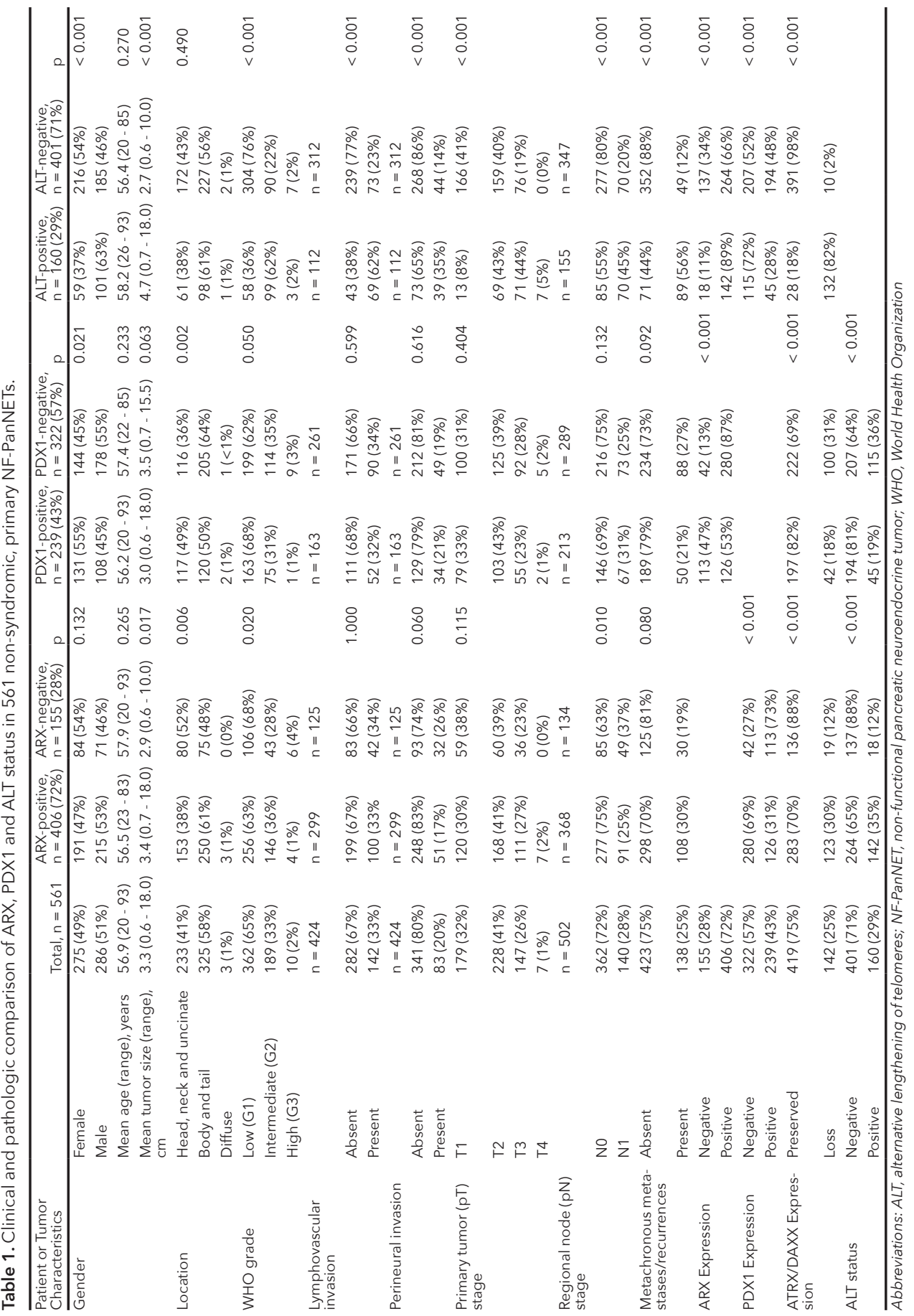




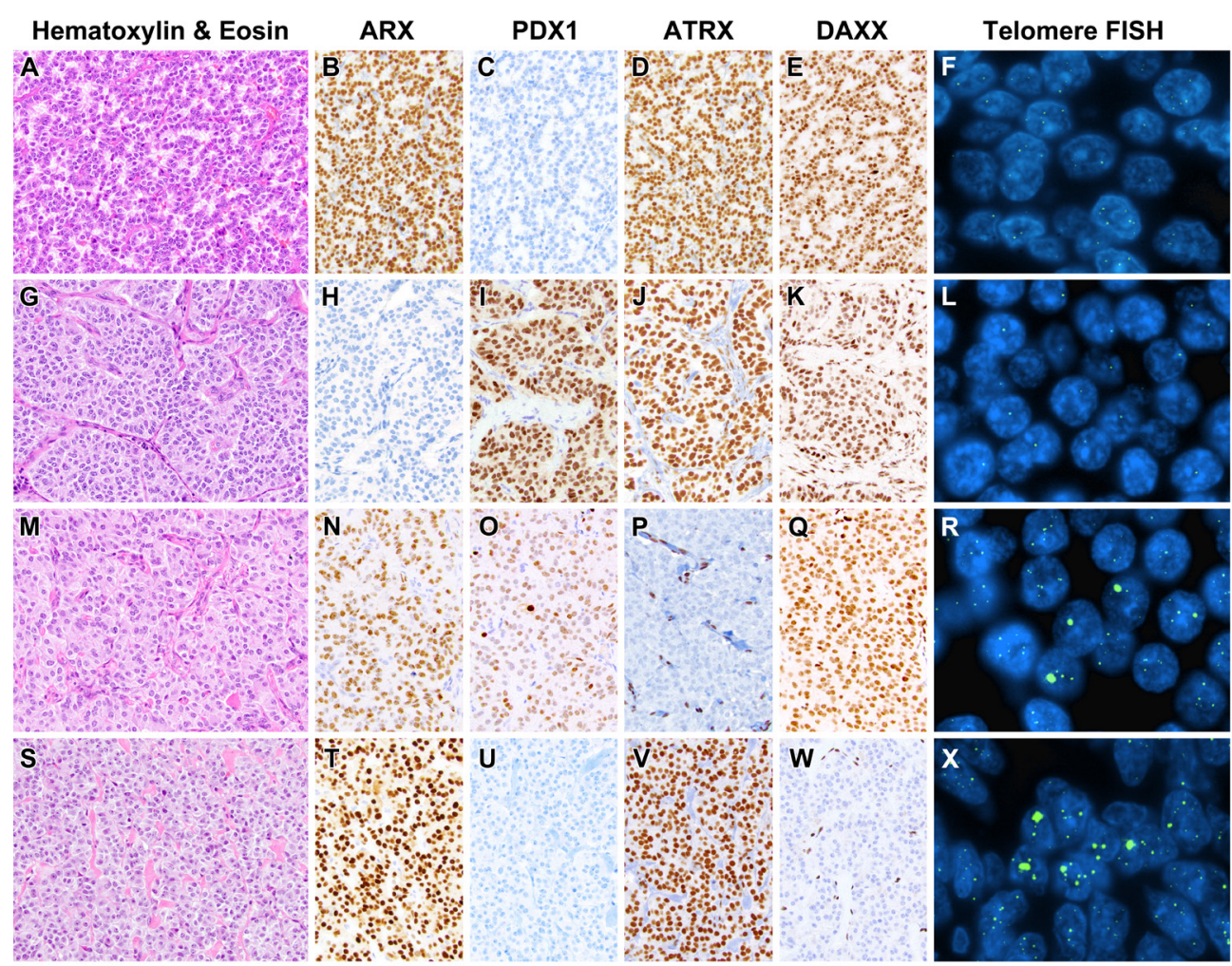

Figure 2. Representative examples of non-functional pancreatic neuroendocrine tumors (NF-PanNETs) that have been assessed by immunolabeling for ARX, PDX, ATRX and DAXX, and telomere-specific fluorescence in situ hybridization (FISH) for ALT. (A) NF-PanNET with positive expression for ARX (B) and negative expression for PDX1 (C), while both ATRX (D) and DAXX (E) exhibited preserved nuclear expression and an absence of alternative lengthening of telomeres (ALT) (F). (G) NF-PanNET with negative expression for ARX (H) and positive expression for PDX1 (I), while both ATRX (J) and DAXX (K) had preserved expression and an absence of ALT (L). (M) NF-PanNET with expression for both ARX (N) and PDX1 (O), but loss of nuclear ATRX (P) and preserved nuclear expression for DAXX (Q). The loss of ATRX expression correlated with the presence of large, ultrabright intranuclear foci by telomere-specific FISH, consistent with ALT (R). (S) NF-PanNET with positive ARX expression (T), negative PDX1 expression (U), preserved ATRX expression (V) and loss of DAXX expression (W). Similar to loss of ATRX, DAXXnegative NF-PanNETs were associated with large, ultrabright telomere signals, consistent with ALT (X).

risk factors by univariate analysis were performed using Cox proportional hazard regression to identify independent risk factors for RFS. Disease-specific survival (DSS) for patients with NF-PanNETs metastases was calculated from the date of primary diagnosis and date of metastatic diagnosis to the date of death due to disease or date of last follow-up (if death did not occur). All statistical analyses were performed using the SPSS Statistical software, version 25 (IBM, Armonk, NY) and statistical significance was defined as a $p$ value of $<0.05$. 

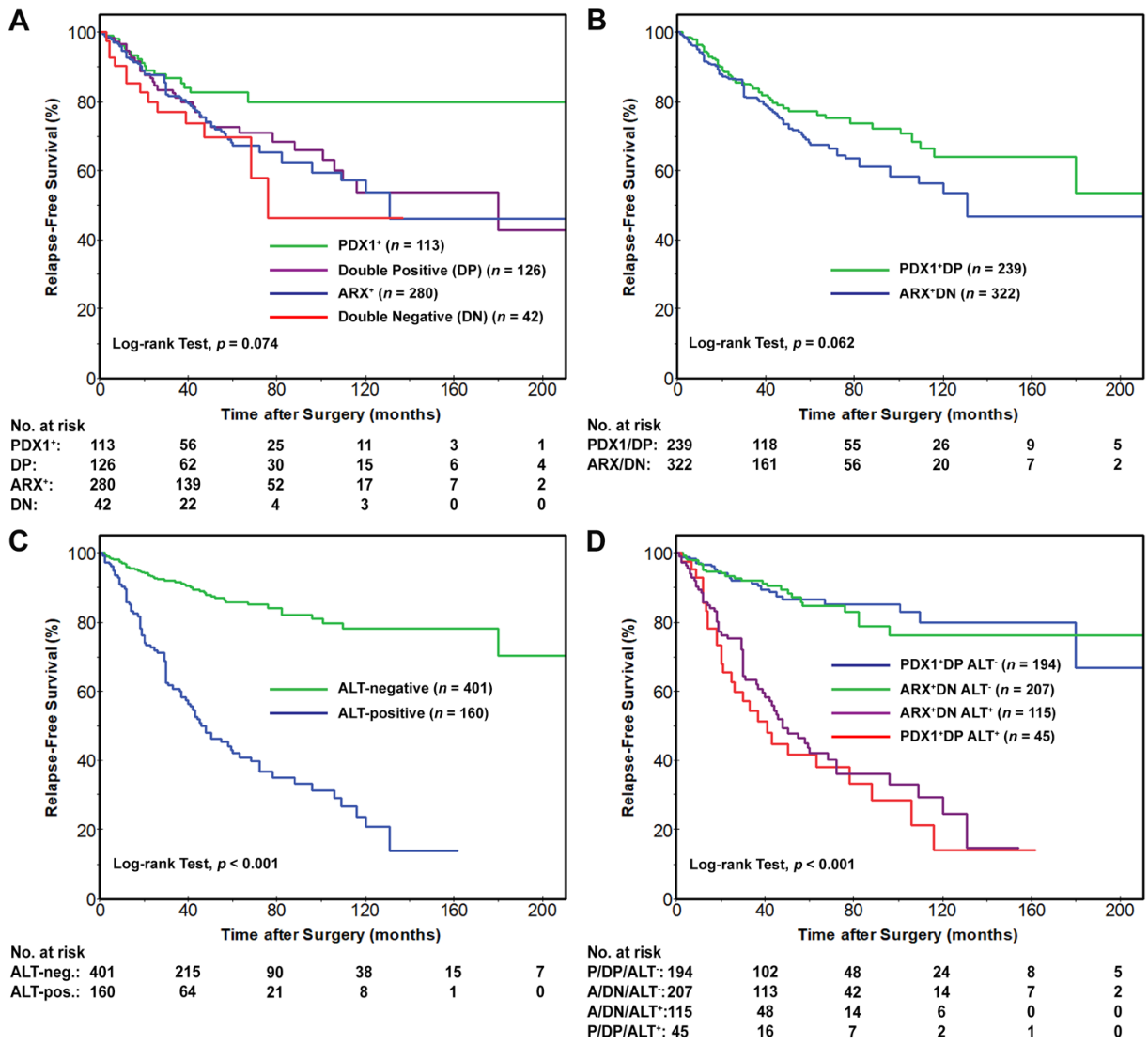

Figure 3. Kaplan-Meier curves comparing relapse-free survival (RFS) after surgical resection for patients with NF-PanNETs. (A) No statistically significant differences in RFS were identified between patients with NF-PanNETs $(n=561)$ that were classified into four immunophenotypic groups: ARX-positive/PDX1-negative (ARX+), ARXnegative/PDX1-positive (PDX+), ARX-positive/PDX1-positive ("double positive", DP), and ARX-negative/PDX1negative ("double negative", DN). (B) Similarly, no RFS difference was seen between patients with ARX++DN NFPanNETs and patients with PDX $1^{+}+$DP NF-PanNETs. However, (C) the RFS for patients with ALT-positive NF-PanNETs was significantly shorter than patients with ALT-negative NF-PanNETs. In addition, (D) the combination of ALT status and the immunophenotypic subgroups, $A R X^{+}+D N$ and PDX $1^{+}+D P$, did not demonstrate any prognostic benefit over ALT status alone.

The Cox proportional hazard assumptions were inspected for each covariate and graphed using scaled Schoenfeld residuals. For each covariate, and each model as a whole, none of the Cox proportional hazard assumptions were violated. These analyses were performed with the survival and surviminer packages for R version 4.0.2. 


\section{RESULTS}

\section{The clinicopathologic features of ARX, PDX1, ATRX/DAXX, and ALT in NF-PanNETs}

Among an international, multi-institutional cohort of 561 non-syndromic and non-functional PanNETs (NF-PanNETs) without distant metastases at surgical resection, nuclear expression for ARX and PDX1 was identified in 406 (72\%) and 239 (43\%) cases (Figure 2), respectively, and summarized in Table 1 and Supplementary Data. Expression of both proteins was present in 126 (22\%) NF-PanNETs and absent in 42 (8\%) NF-PanNETs. In comparison to ARX-negative NF-PanNETs, ARX expression correlated with larger tumor size, pancreatic body/tail location, high WHO grade, negative regional lymph node involvement, loss of ATRX/DAXX immunolabeling, and ALT ( $p<0.050$ for all). PDX1-positive NF-PanNETs were more frequently found in female patients and associated with pancreatic head/uncinate/ neck location, preserved immunolabeling for ATRX/DAXX, and an absence of ALT $(p<0.050$ for all).

Based on ARX and PDX1 status, the NF-PanNET study cohort was subdivided into the following 4 immunophenotypic groups: 280 (50\%) ARX-positive/PDX1-negative, 113 (20\%) ARX-negative/PDX1-positive, 126 (22\%)ARX-positive/PDX1-positive ("double positive", DP), and 42 (8\%) ARX-negative/PDX1-negative ("double negative", DN) cases (Supplementary Table 4). Further, in accordance with Cejas et al.(26), NF-PanNETs classified as ARX-positive/ PDX1-negative, and "double negative" were combined into a single group (ARX++DN, $\mathrm{n}=322$ [57\%]) and summarized in Supplementary Table 5. Likewise, ARX-negative/PDXpositive, and "double positive" NF-PanNETs were categorized together (PDX1++DP, n=239 [43\%]). In relation to PDX1++DP NF-PanNETs, ARX++DN NF-PanNETs were more likely to occur in male patients, show pancreatic body/tail location, have loss of ATRX/DAXX immunolabeling and exhibit $\operatorname{ALT}(p<0.050$ for all).

The presence of ALT and ATRX/DAXX loss in NF-PanNETs was identified in 160 (29\%) and 142 (25\%) cases, respectively (Table 1 and Supplementary Table 6). Further, among the entire study cohort, ALT positivity and/or loss of ATRX/DAXX were detected in 170 (30\%) NF-PanNETs. ALT-positive NF-PanNETs, as compared to ALT-negative NF-PanNETs, were associated with a male predilection, and loss of ATRX/DAXX immunolabeling $(p<0.001$ for all). In addition, ALT-positive NF-PanNETs exhibited larger tumor size, high WHO grade, lymphovascular invasion, perineural invasion, advanced pathologic T-stage, regional lymph node metastases, and metachronous (postoperative) distant metastases/recurrences 
A

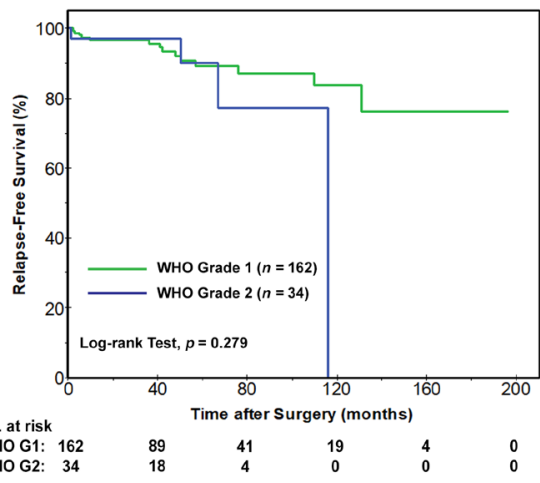

B

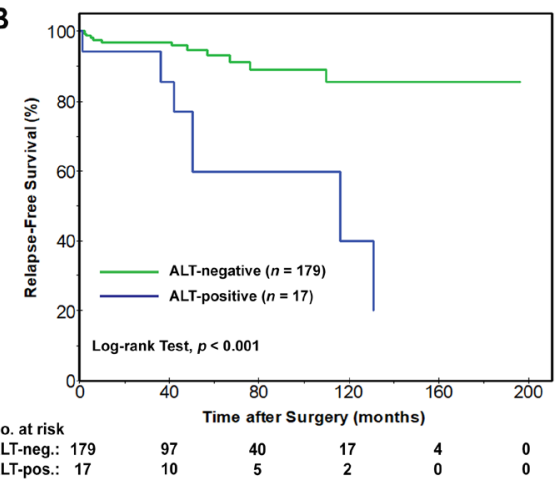

Figure 4. Kaplan-Meier curves for RFS among patients with NF-PanNETs of $\leq 2.0 \mathrm{~cm}$ and without regional lymph node metastases $(n=196)$. Based on the 2017/2019 World Health Organization (WHO) grading system for NFPanNETs, (A) no statistically significant difference in RFS was identified; however, (B) the presence of ALT was associated with shorter RFS.

$(p<0.001$ for all). Similar associations were seen with ATRX/DAXX loss (Supplementary Table 5) and the combination of ALT positivity and/or loss of ATRX/DAXX.

\section{The prognostic significance of ARX, PDX1, ATRX/DAXX, and ALT in NF-PanNETs}

An analysis of relapse-free survival (RFS) revealed no statistically significant differences based on the status of ARX, PDX1, or among the 4 immunophenotypic groups (Figure $3 A)$. Furthermore, no RFS differences were observed between patients with $A R X^{+}+D N$ NF-PanNETs and patients with PDX $1^{+}+$DP NF-PanNETs (Figure 3B). In contrast, the RFS for patients with ALT-positive NF-PanNETs was significantly shorter than patients with ALT-negative NF-PanNETs (Figure 3C). Patient RFS rates associated with ALT-positive NF-PanNETs were $86 \%, 42 \%$ and $21 \%$ at 1,5 and 10 years, respectively, as compared to the ALT-negative NF-PanNETs with $96 \%, 86 \%$ and $78 \%$ at 1,5 and 10 years, respectively $(p<0.001)$. However, the combination of ALT status and the immunophenotypic subgroups, $\mathrm{ARX}^{+}+\mathrm{DN}$ and $\mathrm{PDX} 1^{+}+\mathrm{DP}$, did not demonstrate any additional prognostic relevance over ALT status alone (Figure 3D). Of note, a longer RFS was identified among patients with ARXnegative/PDX1-positive NF-PanNETs as compared to the remaining patients $(p=0.014)$; however, there was a lack of additional prognostic significance upon accounting for ALT (Supplementary Figure 1). Loss of ATRX/DAXX immunolabeling was also associated with shorter RFS with 1-, 5- and 10-year rates of 90\%, $40 \%$ and $20 \%$, respectively, as compared to $96 \%, 85 \%$ and $78 \%$, respectively, for preserved ATRX/DAXX immunolabeling $(p<0.001)$. 


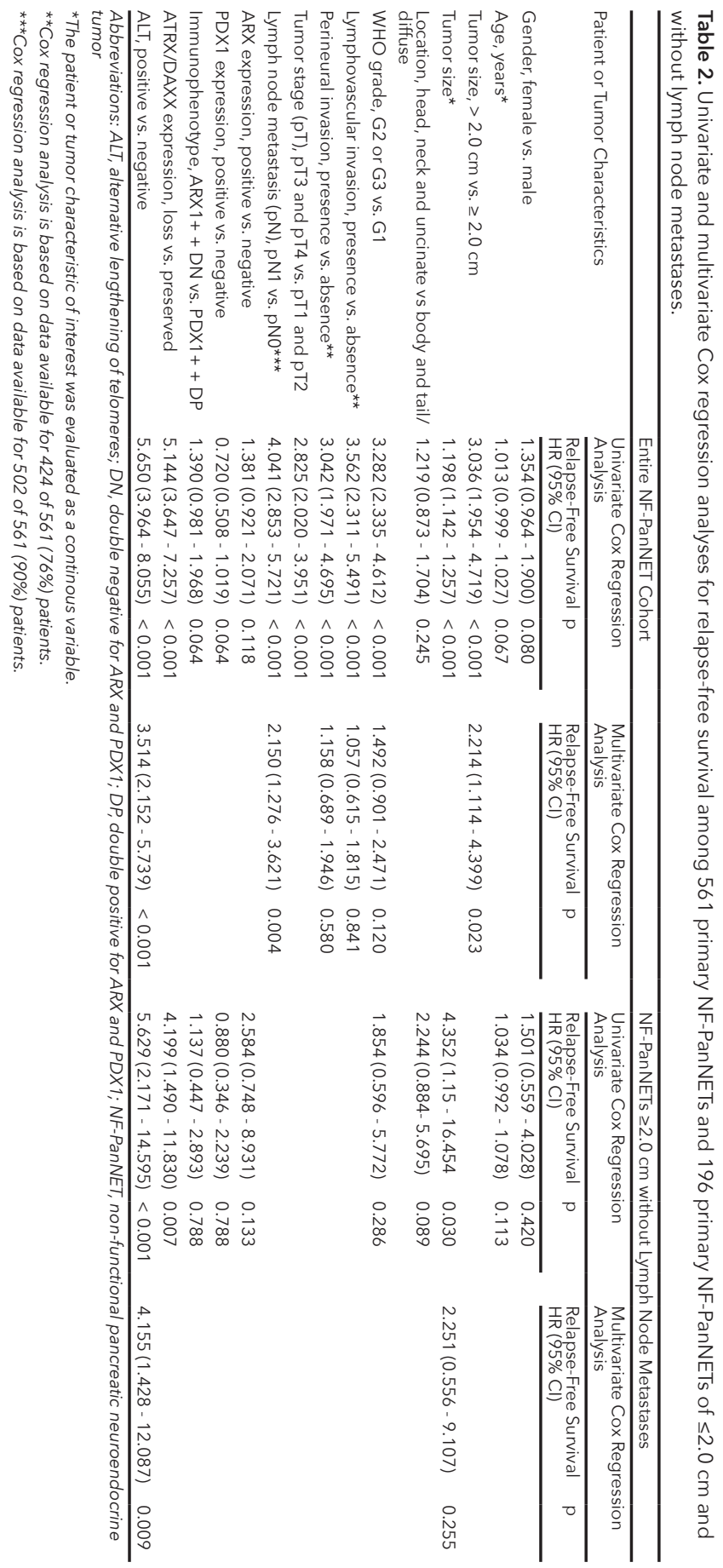


Table 3. Comparison of ARX, PDX1, ATRX/DAXX and ALT status among 1,322 neuroendocrine tumors of the pancreas and other organ sites.

\begin{tabular}{|c|c|c|c|c|c|c|c|}
\hline Tumor type & $\begin{array}{l}\text { Total, } \\
\mathrm{n}=1,322\end{array}$ & $\begin{array}{l}\text { ARX-positive/ } \\
\text { PDX1-negative }\end{array}$ & $\begin{array}{l}\text { ARX-negative/ } \\
\text { PDX1-positive }\end{array}$ & $\begin{array}{l}\text { ARX-positive/ } \\
\text { PDX1-positive }\end{array}$ & $\begin{array}{l}\text { ARX-negative/ } \\
\text { PDX1-negative }\end{array}$ & $\begin{array}{l}\text { Loss of ATRX/ } \\
\text { DAXX }\end{array}$ & $\begin{array}{l}\text { ALT- } \\
\text { positive }\end{array}$ \\
\hline Primary NF-PanNET & 561 & $280(50 \%)$ & $113(20 \%)$ & $126(22 \%)$ & $42(8 \%)$ & $142(25 \%)$ & $160(29 \%)$ \\
\hline NF-PanNET metastases & 107 & $71(66 \%)$ & $22(21 \%)$ & $14(13 \%)$ & $0(0 \%)$ & $66(62 \%)$ & $76(71 \%)$ \\
\hline $\begin{array}{l}\text { Primary pulmonary carcinoid/ } \\
\text { atypical carcinoid }\end{array}$ & 48 & $0(0 \%)$ & $0(0 \%)$ & $0(0 \%)$ & $48(100 \%)$ & $2(4 \%)$ & $2(4 \%)$ \\
\hline $\begin{array}{l}\text { Carcinoid/atypical carcinoid } \\
\text { metastases }\end{array}$ & 28 & $0(0 \%)$ & $0(0 \%)$ & $0(0 \%)$ & $28(100 \%)$ & $0(0 \%)$ & $0(0 \%)$ \\
\hline Primary gastric NET & 32 & $26(82 \%)$ & $2(6 \%)$ & $2(6 \%)$ & $2(6 \%)$ & $0(0 \%)$ & $0(0 \%)$ \\
\hline Gastric NET metastases & 10 & $1(10 \%)$ & $0(0 \%)$ & $0(0 \%)$ & $9(90 \%)$ & $0(0 \%)$ & $0(0 \%)$ \\
\hline Primary duodenal NET & 41 & $3(7 \%)$ & $15(37 \%)$ & $21(51 \%)$ & $2(5 \%)$ & $0(0 \%)$ & $0(0 \%)$ \\
\hline Duodenal NET metastases & 32 & $9(30 \%)$ & $2(7 \%)$ & $19(56 \%)$ & $2(7 \%)$ & $0(0 \%)$ & $0(0 \%)$ \\
\hline Primary ampullary NET & 7 & $3(42 \%)$ & $2(29 \%)$ & $2(29 \%)$ & $0(0 \%)$ & $0(0 \%)$ & $0(0 \%)$ \\
\hline Primary jejunal NET & 35 & $0(0 \%)$ & $0(0 \%)$ & $0(0 \%)$ & $35(100 \%)$ & $0(0 \%)$ & $0(0 \%)$ \\
\hline Jejunal NET metastases & 71 & $0(0 \%)$ & $0(0 \%)$ & $0(0 \%)$ & $71(100 \%)$ & $0(0 \%)$ & $0(0 \%)$ \\
\hline Primary ileal NET & 123 & $0(0 \%)$ & $0(0 \%)$ & $0(0 \%)$ & $123(100 \%)$ & $0(0 \%)$ & $0(0 \%)$ \\
\hline Ileal NET metastases & 151 & $0(0 \%)$ & $0(0 \%)$ & $0(0 \%)$ & $151(100 \%)$ & $0(0 \%)$ & $0(0 \%)$ \\
\hline Primary colonic NET & 8 & $1(12 \%)$ & $0(0 \%)$ & $0(0 \%)$ & $7(88 \%)$ & $0(0 \%)$ & $0(0 \%)$ \\
\hline Colonic NET metastases & 8 & $0(0 \%)$ & $0(0 \%)$ & $0(0 \%)$ & $8(100 \%)$ & $0(0 \%)$ & $0(0 \%)$ \\
\hline Primary rectal NET & 17 & $16(94 \%)$ & $0(0 \%)$ & $1(6 \%)$ & $0(0 \%)$ & $0(0 \%)$ & $0(0 \%)$ \\
\hline Rectal NET metastases & 10 & $10(100 \%)$ & $0(0 \%)$ & $0(0 \%)$ & $0(0 \%)$ & $0(0 \%)$ & $0(0 \%)$ \\
\hline Primary appendiceal NET & 28 & $26(93 \%)$ & $0(0 \%)$ & $2(7 \%)$ & $0(0 \%)$ & $0(0 \%)$ & $0(0 \%)$ \\
\hline Primary gallbladder NET & 2 & $0(0 \%)$ & $0(0 \%)$ & $0(0 \%)$ & $2(100 \%)$ & $0(0 \%)$ & $0(0 \%)$ \\
\hline Gallbladder NET metastases & 3 & $0(0 \%)$ & $0(0 \%)$ & $0(0 \%)$ & $3(100 \%)$ & $0(0 \%)$ & $0(0 \%)$ \\
\hline
\end{tabular}

Abbreviations: ALT, alternative lengthening of telomeres; NET, neuroendocrine tumor; NF-PanNET, non-functional pancreatic neuroendocrine tumor

The combination of ALT-positive and/or ATRX/DAXX-negative NF-PanNETs correlated with shorter RFS rates of $90 \%, 42 \%$ and $21 \%$ at 1,5 and 10 years, respectively, as compared to ALT-negative and ATRX/DAXX positive NF-PanNETs with $97 \%, 87 \%$ and $80 \%$ at 1, 5 and 10 years, respectively $(p<0.001)$.

Results of univariate and multivariate Cox regression analysis for RFS in relation to various clinicopathologic features including ARX, PDX1, ATRX/DAXX, and ALT status are shown in Table 2. By univariate analysis, shorter RFS was associated with tumor size $>2.0 \mathrm{~cm}$, tumor size as a continuous variable, G2-to-G3 WHO grade, lymphovascular invasion, perineural invasion, advanced tumor stage (pT3 and pT4 vs. pT1 and pT2), regional lymph node metastasis, loss of ATRX/DAXX immunolabeling, and the presence of ALT $(p<0.001$ for all). Multivariate analysis was used to determine the prognostic significance of ALT for RFS and included tumor size $>2.0 \mathrm{~cm}$, WHO grade, lymphovascular invasion, perineural invasion, and regional lymph node metastasis. The presence of ALT was an independent prognostic factor for RFS $(p<0.001)$, and similar results were observed with loss of ATRX/ DAXX immunolabeling when substituted for ALT or combined with ALT ( $p<0.001$ for both). 


\section{Prognostic biomarkers for NF-PanNETs $\leq 2.0 \mathrm{~cm}$ and without regional lymph node metastases}

Considering the difficulties in predicting the clinical behavior of NF-PanNETs $\leq 2.0 \mathrm{~cm}$ and without regional lymph node metastases, a separate subanalysis was performed for 196 patients with small $(\leq 2.0 \mathrm{~cm}$ ) NF-PanNETs (Supplementary Table 7). No statistically significant differences in RFS were identified based on the status of ARX, PDX1, four immunophenotypic groups, $A R X^{+}+D N$ and $P D X 1^{+}+D P$, and $W H O$ grade (Figure 4A). Shorter RFS rates were seen with loss of ATRX/DAXX immunolabeling and the presence of ALT. The 1 -, 5- and 10-year RFS rates were $92 \%, 65 \%$ and $32 \%$, respectively, for patients with loss of ATRX/DAXX as compared to $97 \%, 92 \%$ and $85 \%$, respectively, for patients with preserved ATRX/DAXX ( $p=0.003$ ). Similarly, 1-, 5-, and 10-year RFS rates for patients with ALT-positive NF-PanNETs were $94 \%, 60 \%$ and $40 \%$, respectively, as compared to $97 \%, 93 \%$ and $86 \%$, respectively, for patients with ALT-negative NF-PanNETs $(p<0.001)$ (Figure 4B).

By univariate Cox regression analysis of cases $\leq 2.0 \mathrm{~cm}$, tumor size as a continuous variable, loss of ATRX/DAXX immunolabeling, and the presence of ALT ( $p<0.050$ for all) were the only tumor characteristics associated with shorter RFS. Moreover, within a multivariate model that included tumor size and ALT status, the presence of ALT was an independent prognostic factor for RFS $(p=0.009)$ and interchangeable with loss of ATRX/DAXX immunolabeling $(p=0.042)$. Recursive partitioning for tumor size was attempted to determine an optimal prognostic threshold within this subgroup and yielded an optimal $\leq 1.6 \mathrm{~cm}$ cutoff $(p=0.017$ ) (Supplementary Table 8). The 1-, 5- and 10-year RFS rates for patients with NF-PanNETs of $\leq 1.6 \mathrm{~cm}$ were $98 \%, 92 \%$ and $92 \%$, respectively, as compared to $95 \%, 85 \%$ and $61 \%$, respectively, for patients with NF-PanNETs of $>1.6 \mathrm{~cm}$. However, by multivariate analysis, the presence of ALT $(p=0.011)$ and the loss of ATRX/DAXX immunolabeling $(p=0.044)$ were independent prognostic factors, but tumor size of $\leq 1.6 \mathrm{~cm}$ was not significant in either multivariate model.

\section{The status of ARX, PDX1, ATRX/DAXX, and ALT in NF-PanNET metastases, other primary NETs and NET metastases}

Within a separate cohort of 107 NF-PanNET metastases from various distant sites, nuclear expression for ARX and PDX1 expression was identified in 85 (79\%) and 36 (34\%) cases, respectively (Table 3 and Supplementary Data). In comparison to 561 primary NF-PanNETs, the status of ARX and PDX1 in NF-PanNET metastases was not statistically different. Conversely, the presence of ALT was detected in a higher proportion of NF-PanNET 
metastases $(n=76,71 \%)$ as compared to primary NF-PanNETs $(n=160,29 \%[p<0.001])$. A similar correlation was found for ATRX/DAXX loss among 66 (62\%) NF-PanNET metastases and $142(25 \%)$ primary NF-PanNETs $(p<0.001)$.

A clinicopathologic analysis of NF-PanNETs metastases revealed an association between ALT-positive NF-PanNET metastases and male gender, primary pancreatic body/ tail location, positive ARX expression, negative PDX1 expression, loss of ATRX/DAXX expression, and more likely to present as metachronous vs. synchronous disease $(p<0.05)$ (Supplementary Table 9). However, 5- and 10-year disease-specific survival (DSS) rates were longer for patients with ALT-positive NF-PanNET metastases with respect to the time after primary diagnosis ( $72 \%$ and $45 \%$ vs. $51 \%$ and $24 \%$, respective, $p=0.027$ ) and time after diagnosis of metastatic disease $(61 \%$ and $38 \%$ vs. $38 \%$ and $17 \%$, respectively, $p=0.043$ ) (Supplementary Figure 2). A similar statistical association between ATRX/DAXX loss and DSS was not observed.

In addition to primary NF-PanNETs and NF-PanNET metastases, a cohort of 341 primary, non-pancreatic NETs and 313 NET metastases were evaluated for ARX and PDX1 (Table 3 and Supplementary Data). Except for lung, jejunal, ileal and gallbladder NETs, both ARX and PDX1 were expressed in NETs from multiple organ sites (Supplementary Figure 3) but their status did not demonstrate clinicopathologic nor prognostic significance (Supplementary Table 10). In contrast, ALT was detected in only 2 (of 48, 4\%) non-metastatic carcinoids of the lung and both cases exhibited ATRX loss by immunohistochemistry (Supplementary Figure 4). Considering the small number of lung carcinoids that were ALT-positive and showed loss of ATRX, a prognostic analysis could not be performed. None of the remaining primary, non-pancreatic NETs nor NET metastases exhibited ALT or loss of ATRX/DAXX immunolabeling.

\section{DISCUSSION}

Improvements and advancements in pathologic assessment, biochemical analysis, and molecular techniques have led to the investigation of multiple biomarkers to determine prognosis and guide treatment for NF-PanNETs (11, 13, 19, 34-36). Whilst some biomarkers, such as mitotic count and Ki-67 proliferation index, have been incorporated into the routine evaluation of NF-PanNETs, most are not used in clinical practice as they have been inadequately studied in patient specimens as to demonstrate their prognostic value. Using a large, international, multi-institutional cohort of NF-PanNETs from patients without 
a germline predisposition syndrome or distant metastases at clinical presentation, we validated the utility of ATRX/DAXX by immunolabeling and ALT by telomere-specific FISH as prognostic biomarkers. Loss of ATRX/DAXX and the presence of ALT correlated with known adverse prognostic features for NF-PanNETs, such as large tumor size, high WHO grade, lymphovascular invasion, perineural invasion, advanced pathologic T-stage, and regional lymph node metastases. Importantly, ATRX/DAXX loss and ALT were independent prognostic biomarkers for shorter RFS among patients with NF-PanNETs.

While DNA repair and recombination have been implicated in ALT, the exact mechanisms underlying this telomere maintenance program are still being elucidated (37, 38). However, ALT frequently coincides with alterations in ATRX and/or DAXX (21). Inactivation of ATRX and/ or DAXX are late genomic events in the pathogenesis of NF-PanNETs and supported by the identification of intratumoral heterogeneity for both proteins and ALT.(24) The prognostic findings presented herein are consistent with previous studies evaluating ATRX/DAXX and ALT $(18,22-24)$. Nonetheless, these reports have included diverse patient populations and, in certain aspects, are potentially of limited clinical relevance in demonstrating the utility of a NF-PanNET prognostic biomarker. For example, prior publications have not specifically addressed patients with NF-PanNETs of $\leq 2.0 \mathrm{~cm}$ and without regional lymph node metastases. These neoplasms are frequently identified incidentally by radiographic imaging and represent a clinical dilemma as to whether surgical management or continued surveillance is indicated. Based on our findings, the loss of ATRX/DAXX and the presence of ALT are associated with shorter RFS for NF-PanNET patients with $\leq 2.0 \mathrm{~cm}$ tumors and without regional lymph node metastases. Moreover, ATRX/DAXX loss and ALT positivity are independent, negative prognostic biomarkers for this subset of neoplasms. Given the large number of patients analyzed across multiple geographic regions, our study seems sufficient to consider incorporating the status of ATRX/DAXX and/or ALT to the routine evaluation of NF-PanNETs and potentially the preoperative assessment of patients with small $(\leq 2.0 \mathrm{~cm})$ NF-PanNETs using cytopathologic cell block material and/or core needle biopsies.(32, 39) In support of the prognostic significance of ATRX/DAXX loss and ALT positivity among primary NF-PanNETs, both biomarkers were detected at a higher prevalence in NF-PanNET metastases. While the presence of ALT in NF-PanNETs correlated with the development of distant metastases, an interesting quandary was an improved DSS for patients with ALTpositive NF-PanNET metastases as compared to patients with ALT-negative NF-PanNET metastases. At first glance, these findings are seemingly contradictory; however, the results are in agreement with previous studies. Jiao et al. and Dogeas et al. both reported PanNET 
patients with ALT-positive liver metastases had a longer survival time and Kim et al. observed that patients with ALT-positive primary tumors had better overall survival $(16,23,40)$. Thus, the prognostic utility of ALT in NF-PanNETs may vary based on the disease context.

Except for 2 (4\%) pulmonary carcinoids, the loss of ATRX/DAXX and the presence of ALT were not seen in other primary non-pancreatic NETs and NET metastases. Among non-pancreatic NETs, a relative paucity of ATRX and/or DAXX genomic alterations have been reported by whole exome sequencing and whole genomic sequencing studies (41-44). Thus, ATRX/ DAXX loss and ALT may also serve as diagnostic biomarkers to suggest pancreatic origin for NET metastases where a primary site cannot be clinically determined; but, requires further studies to confirm these findings (45). Additionally, our results indicate that ATRX/DAXX loss and ALT activation play important roles in the pathogenesis of NF-PanNETs, although not in NETs from other organ sites. In contrast, ARX and PDX1 are differentially expressed in gastric, duodenal, ampullary, colonic, rectal, and appendiceal NETs. These findings are not surprising as both ARX and PDX1 are critical to the development of not only the islets of Langerhans, but also enteroendocrine cells of the gastrointestinal tract (27). Interestingly, jejunal and ileal NETs were consistently negative for ARX and PDX1, while duodenal and ampullary NETs were positive for either transcription factor in $94 \%$ of cases.

In addition to the status of ATRX/DAXX and ALT, ARX and PDX1 have also been reported to be prognostic biomarkers for NF-PanNETs. Recently, Cejas et al. reported that locoregional recurrences and distant metastases almost exclusively occurred in patients with ARX+PDX1- and "double negative" (DN) NF-PanNETs (26). However, the authors' patient cohort was enriched for patients with MEN1 syndrome. The absence of MEN1 patients within our study may explain the lack of statistical significance observed in the incidence of postoperative distant metastases/recurrences and RFS based on ARX++DN status and PDX1 expression (PDX1++DP). In fact, we found neither subgroup was associated with known adverse prognostic pathologic features for NF-PanNETs. Expression of ARX in NFPanNETs, regardless of PDX1 status, did correlate with large tumor size, high WHO grade, the presence of regional lymph node metastases, ALT positivity and loss of ATRX/DAXX; however, ARX expression was not associated with RFS in neither our full study cohort nor the subcohort of $\leq 2.0 \mathrm{~cm}$ NF-PanNETs without lymph node metastases.

It is worth noting that there are a few limitations to our study. This study represents one of the largest series of NF-PanNETs to be prognostically analyzed for molecular biomarkers, 
but it is still retrospective in design and may suffer from surgical selection bias. In addition, although regional lymph node metastases can be challenging to detect preoperatively, not all patients had sufficient regional lymph nodes for evaluation. Among patients with primary NF-PanNETs, $11 \%$ underwent a limited pancreatectomy procedure (e.g., enucleation or central pancreatectomy), and, as a result, regional lymphadenectomy was suboptimal for these patients. Enucleation and central pancreatectomy procedures are typically done in the setting of small NF-PanNETs $(\leq 2.0 \mathrm{~cm})$ because most of these tumors have an indolent clinical course. Within our study cohort, 18 of 196 (9\%) of small NF-PanNETs developed postoperative distant metastases/recurrences and $28 \%$ and $39 \%$ of these cases were ATRX/ DAXX-negative and ALT-positive, respectively. In comparison, loss of ATRX/DAXX and the presence of ALT were identified in only $5 \%$ and $6 \%$, respectively, of non-metastatic, small NF-PanNETs. Thus, incorporating the status of ATRX/DAXX and ALT among $\leq 2.0 \mathrm{~cm}$ NF-PanNETs without regional lymph node metastases could aid in triaging patients for appropriate surgical management to ensure complete regional lymph node dissection. Additionally, the thresholds used for ARX and PDX1 immunolabeling within our study may not be the same as those used by Cejas et al. (26). Herein, we defined positive expression at a cutoff of $>10 \%$ immunolabeling of neoplastic nuclei. While this may partially explain the discrepancy between the results of these two studies, the proportion of ARX+, PDX1+, DP and DN NF-PanNETs within our cohort was 50\%, 20\%, 22\% and $8 \%$, respectively, and similar to the cohort reported by Cejas et al of $43 \%, 19 \%, 28 \%$ and $10 \%$, respectively (26). Furthermore, ARX and PDX1 cannot be discounted as prognostic markers and may be useful in the setting of MEN1 syndrome. Finally, we used tissue microarrays (TMAs) to evaluate a subset of NF-PanNETs and NETs from other organ sites. As a TMA consists of "punches" of tumor tissue, each sample may not be representative of the entire tumor as compared to whole tissue sections. However, the potentially scant amount of tumor in TMAs can be beneficial at it allows for simulation of needle core biopsies, and, hence, useful in determining the utility of prognostic and diagnostic biomarkers by mimicking preoperative specimens. Moreover, there were no statistically significant differences between NF-PanNETs evaluated using TMAs and NF-PanNETs evaluated using whole sections. It is also important to underscore that immunolabeling for ATRX and DAXX and telomere-specific FISH for ALT were performed at multiple institutions and the results were consistent across individual sites. Hence, our study further demonstrates the broad feasibility of implementing these biomarkers into clinical practice. 
In summary, we report the comprehensive assessment of ARX, PDX1, ATRX, DAXX, and ALT in a large, international and multi-institutional cohort of non-syndromic NF-PanNETs without synchronous distant metastases. Although the expression of ARX correlated with known adverse prognostic features, such as larger tumor size and high WHO grade, there was a lack of association with RFS. Comparatively, NF-PanNETs with ALT positivity or ATRX/DAXX loss showed several adverse prognostic factors and these patients had shorter RFS. Both the presence of ALT and loss of ATRX/DAXX in NF-PanNETs were negative, independent prognostic biomarkers for RFS including patients with small NF-PanNETs $(\leq 2.0 \mathrm{~cm}$ ), which represent a clinical dilemma for surgical management. Moreover, analyzing a cohort of $>1,300$ pancreatic and non-pancreatic NETs and metastases, the presence of ALT and loss of ATRX/DAXX were highly specific, diagnostic biomarkers of pancreatic origin. While further prospective studies are warranted, our findings underscore the utility of ALT and ATRX/DAXX as prognostic biomarkers for the preoperative and postoperative evaluation of NF-PanNETs, and, in the setting of a NET metastasis of unknown origin, as highly specific diagnostic biomarkers to indicate the possibility of a pancreatic primary.

\section{ACKNOWLEDGEMENTS}

The authors would like to thank Mrs. Kate Smith and Mrs. Lynn Wolkenstein for outstanding administrative assistance. In addition, the authors thank Drs. Ralph H. Hruban and Matthew H. Kulke for helpful comments and suggestions.

\section{DISCLOSURE/CONFLICT OF INTEREST}

Dr. Singhi has received an honorarium from Foundation Medicine, Inc. The remaining authors have disclosed that they have no financial interests, arrangements, affiliations, or commercial interests with the manufacturers of any products discussed in this article or their competitors.

\section{FUNDING}

This study was supported in part by grants from the Dutch Digestive Foundation/Maag Lever Darm Stichting (CDG 14-020) (to W.M. Hackeng. and L.A.A. Brosens); Associazione Italiana Ricerca Cancro (AIRC 5×1000 n. 12182 and Start up n. 18718), European Community ERANET PMTR-pNET (cod. D18TR5, B46C17000260001), Italian Ministry of Health (FIMPCUP_J38D19000690001), Fondazione Cariverona: Oncology Biobank Project (prot. 203885/2017) (to A. Scarpa); NRF-2016R1A2B4009381 from the National Research 
Foundation of Korea (to S.-M. Hong); the Basic/Translational Science Investigator Award from the North American Neuroendocrine Tumor Society supported by the Neuroendocrine Tumor Research Foundation (to C. M Heaphy); National Pancreas Foundation, Sky Foundation and the Pittsburgh Liver Research Center at the University of Pittsburgh (NIH/ NIDDK P30DK120531) (to A. D. Singhi).

\section{REFERENCES}

1. Dasari A, Shen C, Halperin D, Zhao B, Zhou S, Xu Y, et al. Trends in the Incidence, Prevalence, and Survival Outcomes in Patients With Neuroendocrine Tumors in the United States. JAMA Oncol. 2017;3(10):1335-42.

2. Howlader N, Noone AM, Krapcho M, Miller D, Brest A, Yu M, et al. SEER Cancer Statistics Review, 1975-2017 Bethesda, MD: National Cancer Institute; 2019 [Available from: https://seer. cancer.gov/csr/1975_2017/.

3. Zhang IY, Zhao J, Fernandez-Del Castillo C, Braun Y, Razmdjou S, Warshaw AL, et al. Operative Versus Nonoperative Management of Nonfunctioning Pancreatic Neuroendocrine Tumors. J Gastrointest Surg. 2016;20(2):277-83.

4. Sadot E, Reidy-Lagunes DL, Tang LH, Do RK, Gonen M, D'Angelica MI, et al. Observation versus Resection for Small Asymptomatic Pancreatic Neuroendocrine Tumors: A Matched Case-Control Study. Ann Surg Oncol. 2016;23(4):1361-70.

5. Network NCC. Neuroendocrine and adrenal tumors (Version 1.2019) 2019 [Available from: https://www.nccn.org/ professionals/physician_gls/pdf/neuroendocrine.pdf.

6. Falconi M, Eriksson B, Kaltsas G, Bartsch DK, Capdevila J, Caplin M, et al. ENETS Consensus Guidelines Update for the Management of Patients with Functional Pancreatic Neuroendocrine Tumors and Non-Functional Pancreatic Neuroendocrine Tumors. Neuroendocrinology. 2016;103(2):153-71.

$7 . \quad H o w e$ JR, Merchant NB, Conrad C, Keutgen XM, Hallet J, Drebin JA, et al. The North American Neuroendocrine Tumor Society Consensus Paper on the Surgical Management of Pancreatic Neuroendocrine Tumors. Pancreas. 2020;49(1):1-33.

8. Haynes AB, Deshpande V, Ingkakul T, Vagefi PA, Szymonifka J, Thayer SP, et al. Implications of incidentally discovered, nonfunctioning pancreatic endocrine tumors: short-term and long-term patient outcomes. Arch Surg. 2011;146(5):534-8.

9. Kuo EJ, Salem RR. Population-level analysis of pancreatic neuroendocrine tumors $2 \mathrm{~cm}$ or less in size. Ann Surg Oncol. 2013;20(9):2815-21.

10. Cherenfant J, Stocker SJ, Gage MK, Du H,

Thurow TA, Odeleye M, et al. Predicting aggressive behavior in nonfunctioning pancreatic neuroendocrine tumors. Surgery. 2013;154(4):785-91; discussion 91-3.

11. Pea A, Yu J, Marchionni L, Noe M, Luchini C, Pulvirenti A, et al. Genetic Analysis of Small Well-differentiated Pancreatic Neuroendocrine Tumors Identifies Subgroups With Differing Risks of Liver Metastases. Ann Surg. 2020;271(3):566-73.

12. Lloyd RV, Osamura RY, Klöppel G, Rosai J. WHO Classification of Tumours of Endocrine Organs. 4th ed. Lyon, France: IARC Press; 2017.
13. McCall CM, Shi C, Cornish TC, Klimstra DS, Tang LH, Basturk $\mathrm{O}$, et al. Grading of well-differentiated pancreatic neuroendocrine tumors is improved by the inclusion of both Ki67 proliferative index and mitotic rate. Am J Surg Pathol. 2013;37(11):1671-7. 14. Reid MD, Bagci P, Ohike N, Saka B, Erbarut Seven I, Dursun N, et al. Calculation of the Ki67 index in pancreatic neuroendocrine tumors: a comparative analysis of four counting methodologies. Mod Pathol. 2016;29(1):93.

15. Hwang HS, Kim Y, An S, Kim SJ, Kim JY, Kim SY, et al. Grading by the Ki-67 Labeling Index of Endoscopic Ultrasound-Guided Fine Needle Aspiration Biopsy Specimens of Pancreatic Neuroendocrine Tumors Can Be Underestimated. Pancreas. 2018;47(10):1296-303.

16. Jiao Y, Shi C, Edil BH, de Wilde RF, Klimstra DS, Maitra A, et al. DAXX/ATRX, MEN1, and mTOR pathway genes are frequently altered in pancreatic neuroendocrine tumors. Science. 2011;331(6021):1199-203.

17. Scarpa A, Chang DK, Nones K, Corbo V, Patch AM, Bailey $P$, et al. Whole-genome landscape of pancreatic neuroendocrine tumours. Nature. 2017;543(7643):65-71.

18. Roy S, LaFramboise WA, Liu TC, Cao D, Luvison A, Miller $C$, et al. Loss of Chromatin-Remodeling Proteins and/or CDKN2A Associates With Metastasis of Pancreatic Neuroendocrine Tumors and Reduced Patient Survival Times. Gastroenterology. 2018;154(8):2060-3 e8.

19. Hong X, Qiao S, Li F, Wang W, Jiang R, Wu H, et al Whole-genome sequencing reveals distinct genetic bases for insulinomas and non-functional pancreatic neuroendocrine tumours: leading to a new classification system. Gut. 2020;69(5):877-87.

20. Di Domenico A, Pipinikas CP, Maire RS, Brautigam K, Simillion C, Dettmer MS, et al. Epigenetic landscape of pancreatic neuroendocrine tumours reveals distinct cells of origin and means of tumour progression. Commun Biol. 2020;3(1):740.

21. Heaphy CM, de Wilde RF, Jiao Y, Klein AP, Edil BH,

Shi $C$, et al. Altered telomeres in tumors with ATRX and DAXX mutations. Science. 2011;333(6041):425.

22. Marinoni I, Kurrer AS, Vassella E, Dettmer M, Rudolph T, Banz V, et al. Loss of DAXX and ATRX are associated with chromosome instability and reduced survival of patients with pancreatic neuroendocrine tumors. Gastroenterology. 2014;146(2):453-60 e5.

23. Kim JY, Brosnan-Cashman JA, An S, Kim SJ, Song KB, Kim MS, et al. Alternative Lengthening of Telomeres in Primary Pancreatic Neuroendocrine Tumors Is Associated with Aggressive Clinical Behavior and Poor Survival. Clin Cancer Res. 2017;23(6):1598-606. 


\section{Chapter 8}

24. Singhi AD, Liu TC, Roncaioli JL, Cao D, Zeh HJ

Zureikat $\mathrm{AH}$, et al. Alternative Lengthening of Telomeres and Loss of DAXX/ATRX Expression Predicts Metastatic Disease and Poor Survival in Patients with Pancreatic Neuroendocrine Tumors. Clin Cancer Res. 2017;23(2):600-9.

25. Chan CS, Laddha SV, Lewis PW, Koletsky MS, Robzyk K, Da Silva E, et al. ATRX, DAXX or MEN1 mutant pancreatic neuroendocrine tumors are a distinct alpha-cell signature subgroup. Nat Commun. 2018;9(1):4158.

26. Cejas P, Drier Y, Dreijerink KMA, Brosens LAA, Deshpande $V$, Epstein $C B$, et al. Enhancer signatures stratify and predict outcomes of non-functional pancreatic neuroendocrine tumors. Nat Med. 2019;25(8):1260-5.

27. Du A, McCracken KW, Walp ER, Terry NA, Klein TJ, Han $A$, et al. Arx is required for normal enteroendocrine cell development in mice and humans. Dev Biol. 2012;365(1):175-88. 28. Hackeng WM, Schelhaas W, Morsink FHM, Heidsma $\mathrm{CM}$, van Eeden S, Valk GD, et al. Alternative Lengthening of Telomeres and Differential Expression of Endocrine Transcription Factors Distinguish Metastatic and Non-metastatic Insulinomas. Endocr Pathol. 2020;31(2):108-18.

29. Corbo V, Dalai I, Scardoni M, Barbi S, Beghelli S, Bersani S, et al. MEN1 in pancreatic endocrine tumors: analysis of gene and protein status in 169 sporadic neoplasms reveals alterations in the vast majority of cases. Endocr Relat Cancer. 2010;17(3):771-83.

30. Rindi G, Klimstra DS, Abedi-Ardekani B, Asa SL, Bosman FT, Brambilla E, et al. A common classification framework for neuroendocrine neoplasms: an International Agency for Research on Cancer (IARC) and World Health Organization (WHO) expert consensus proposal. Mod Pathol. 2018;31(12):1770-86. 31. Amin MB, Edge SB, Green F, Byrd DR, Brookland RK, Washington MK, et al. AJCC Cancer Staging Manual. Cancer AJCo, editor: Springer International Publishing; 2017. 32. Hackeng WM, Morsink FHM, Moons LMG, Heaphy CM, Offerhaus GJA, Dreijerink KMA, et al. Assessment of ARX expression, a novel biomarker for metastatic risk in pancreatic neuroendocrine tumors, in endoscopic ultrasound fine-needle aspiration. Diagn Cytopathol. 2020;48(4):308-15.

33. Cesare AJ, Heaphy CM, O'Sullivan RJ. Visualization of Telomere Integrity and Function In Vitro and In Vivo Using Immunofluorescence Techniques. Curr Protoc Cytom. 2015;73:12 $401-31$.

34. Ellison TA, Wolfgang CL, Shi C, Cameron JL, Murakami P, Mun LJ, et al. A single institution's 26-year experience with nonfunctional pancreatic neuroendocrine tumors: a validation of current staging systems and a new prognostic nomogram. Ann
Surg. 2014;259(2):204-12

35. Fung AD, Cohen C, Kavuri S, Lawson D, Gao X, Reid MD. Phosphohistone $\mathrm{H} 3$ and $\mathrm{Ki}-67$ labeling indices in cytologic specimens from well-differentiated neuroendocrine tumors of the gastrointestinal tract and pancreas: a comparative analysis using automated image cytometry. Acta Cytol. 2013;57(5):501-8. 36. Estrella JS, Broaddus RR, Mathews A, Milton DR, Yao JC, Wang $\mathrm{H}$, et al. Progesterone receptor and PTEN expression predict survival in patients with low- and intermediate-grade pancreatic neuroendocrine tumors. Arch Pathol Lab Med. 2014;138(8):1027-36.

37. Dilley RL, Verma P, Cho NW, Winters HD, Wondisford AR, Greenberg RA. Break-induced telomere synthesis underlies alternative telomere maintenance. Nature. 2016;539(7627):54-8. 38. Zhang JM, Yadav T, Ouyang J, Lan L, Zou L. Alternative Lengthening of Telomeres through Two Distinct Break-Induced Replication Pathways. Cell Rep. 2019;26(4):955-68 e3. 39. VandenBussche CJ, Allison DB, Graham MK, Charu $\mathrm{V}$, Lennon AM, Wolfgang $C L$, et al. Alternative lengthening of telomeres and ATRX/DAXX loss can be reliably detected in FNAs of pancreatic neuroendocrine tumors. Cancer Cytopathol. 2017; 125(7):544-51.

40. Dogeas E, Karagkounis G, Heaphy CM, Hirose K, Pawlik TM, Wolfgang CL, et al. Alternative lengthening of telomeres predicts site of origin in neuroendocrine tumor liver metastases. $J$ Am Coll Surg. 2014;218(4):628-35.

41. Karpathakis A, Dibra H, Pipinikas C, Feber A, Morris T, Francis J, et al. Prognostic Impact of Novel Molecular Subtypes of Small Intestinal Neuroendocrine Tumor. Clin Cancer Res. 2016;22(1):250-8.

42. Francis JM, Kiezun A, Ramos AH, Serra S, Pedamallu CS, Qian ZR, et al. Somatic mutation of CDKN1B in small intestine neuroendocrine tumors. Nat Genet. 2013;45(12):1483-6.

43. Simbolo M, Mafficini A, Sikora KO, Fassan M, Barbi S, Corbo $V$, et al. Lung neuroendocrine tumours: deep sequencing of the four World Health Organization histotypes reveals chromatin-remodelling genes as major players and a prognostic role for TERT, RB1, MEN1 and KMT2D. J Pathol. 2017;241(4):488-500. 44. Asiedu MK, Thomas CF, Jr., Dong J, Schulte SC, Khadka P, Sun Z, et al. Pathways Impacted by Genomic Alterations in Pulmonary Carcinoid Tumors. Clin Cancer Res. 2018;24(7):1691704.

45. Hackeng WM, Dreijerink KMA, de Leng WWJ,

Morsink FH, Valk GD, Vriens MR, et al. Genome methylation accurately predicts neuroendocrine tumor origin - an online tool. Clin Cancer Res. 2020. 


\section{SUPPLEMENTARY MATERIALS AND METHODS}

\section{Immunohistochemistry}

Immunohistochemistry for ARX, PDX1, ATRX and DAXX was performed manually for 305 primary NF-PanNET using TMAs. Deparaffinized TMA slides were treated with serial xylene treatments and subjected to antigen retrieval using either heated citrate or ethylenediaminetetraacetic acid solution. Blocking of endogenous peroxidase activity was performed prior or after antigen retrieval with $0.6 \% \mathrm{H} 2 \mathrm{O} 2$ (Merck) in methanol for 15 min. Upon treatment for non-specific binding, TMA sections were incubated with primary antibodies for 1 hour at room temperature or overnight at 4oC, followed by secondary antibody (Leica Microsystems or Immunologic) for $30 \mathrm{~min}$ and detected with 3,30-diaminobenzidine (Sigma Aldrich or Immunologic) for $10 \mathrm{~min}$. Washing steps were performed with phosphate buffered saline containing $0.1 \%$ Tween-20 for at least 5 min. Sections were counterstained with hematoxylin, dehydrated, and mounted.

\section{Fluorescence in situ hybridization (FISH)}

Telomere-specific FISH was performed as previously described (1-4). In brief, TMA sections were cut at $4-u m$, incubated for $30 \mathrm{~min}$ at $55 / 56 \circ \mathrm{C}$ or $10 \mathrm{~min}$ at $65 \circ \mathrm{C}$, washed three times for $5 \mathrm{~min}$ in xylene, rinsed in successive 100\%, 95\%, and 70\% ethanol baths, and washed in double-distilled $\mathrm{H} 2 \mathrm{O}$ and, for a subset of cases, $1 \%$ Tween before being placed in antigen unmasking solution in a boiling steamer for 20 to $30 \mathrm{~min}$. Next, slides were rinsed in double-distilled $\mathrm{H} 2 \mathrm{O}$ and dehydrated in successive ethanol washes of $70 \%, 95 \%$, and $100 \%$. Slides were incubated at 72 oC for 10 min or $84 \circ \mathrm{C}$ for 5 min with a fluorescently tagged (either Cy3 or Alexa-488) telomeric-C peptide nucleic acid (PNA) probe complementary to the mammalian telomere repeat sequence ([ $\mathrm{N}$-terminus to C-terminus] CCCTAACCCTAACCCTAA) and hybridized overnight in a dark humidified chamber. Following post-hybridization washes, the slides were counterstained with DAPI (Sigma Aldrich) and mounted with prolong anti-fade mounting medium. Finally, slides were analyzed on a fluorescent light microscope.

\section{SUPPLEMENTARY DATA}

Primary non-functional pancreatic neuroendocrine tumor study cohort

The study cohort consisted of 561 patients with a non-syndromic and non-functional, solitary pancreatic neuroendocrine tumor (NF-PanNET) treated by pancreatectomy with curative intent. No synchronous distant metastases were identified the time of surgical resection. 
Patients ranged in age from 20 to 93 years (mean, 56.9; median, 57.0 years) with a slight predominance in male gender (286 of 561, 51\%). The tumors were predominantly located within the pancreatic body and tail $(n=325,58 \%)$ and ranged in size from 0.6 to $18 \mathrm{~cm}$ (mean, $3.3 \mathrm{~cm}$; median, $2.6 \mathrm{~cm}$ ). Although all PanNETs were morphologically well-differentiated, on the basis of mitotic rate and Ki-67 proliferation index, PanNETs were classified into the following WHO grades: 362 (65\%) grade 1 (G1), 189 (33\%) grade 2 (G2), and 10 (2\%) grade 3 (G3). Data regarding lymphovascular and perineural invasion was available for 424 (76\%) cases and present for 142 (of 424,33\%) and 83 (20\%) tumors, respectively. Using the AJCC prognostic staging system (eighth edition), the NF-PanNETs were classified into the following pathologic tumor (pT) stages: 179 (32\%) pT1, 228 (41\%) pT2, 147 (26\%) pT3 and 7 (1\%) pT4. Regional lymph nodes were submitted for histologic evaluation in 502 (89\%) cases with involvement of 140 (of 502, 28\%) cases. Follow-up information was available for all patients and postoperative (metachronous) distant metastases/recurrences were identified in 138 (25\%) patients. The relapse-free survival (RFS) rates for this study cohort were $95 \%$ at 1 year, $72 \%$ at 5 years and $60 \%$ at 10 years. Of note, no statistically significant clinicopathologic differences were identified among patients from each of the submitting institutions (Supplementary Tables 1, 2 and 3).

In addition to immunohistochemistry for ARX and PDX1, and telomere-specific FISH for ALT, ATRX and DAXX immunolabeling were performed for all 561 NF-PanNETs. Loss of nuclear expression for ATRX, DAXX or both was identified in 56 (10\%), 73 (13\%), and 14 (3\%) NF-PanNETs, respectively. Intratumoral heterogeneity or heterogeneous staining was identified for 3 cases and was only detected among whole tumor sections. These cases consisted of 2 NF-PanNETs with loss of ATRX and 1 NF-PanNET with loss of DAXX. Heterogeneous staining was characterized by a clear presence of two distinct populations of tumor cells demonstrating preserved and loss of nuclear staining for the aforementioned protein. Based on the status of ATRX/DAXX, NF-PanNETs with loss of ATRX/DAXX correlated with a male predilection $(p=0.026)$, larger tumor size $(p<0.001)$, high WHO grade $(p<0.001)$, lymphovascular invasion $(p<0.001)$, perineural invasion ( $p$ $<0.001)$, advanced pathologic T-stage $(p<0.001)$, regional lymph node metastases $(p<$ 0.001 ), metachronous (postoperative) distant metastases $(p<0.001)$ and the presence of $\operatorname{ALT}(p<0.001$ ) (Supplementary Table 5). Of note, among the 138 cases that developed metachronous distant metastases/recurrences, 81 (59\%) patients had loss of ATRX/DAXX within their primary NF-PanNET. Similarly, 89 (65\%) patients had ALT-positive primary NFPanNETs (Table 1). 


\section{NF-PanNET metastases and non-pancreatic neuroendocrine tumor study cohort}

Distant metastases from 107 patients with NF-PanNETs had sufficient pathologic material for further ancillary studies (Table 3). Fourteen (13\%) patients had synchronous metastases, 48 (45\%) had metachronous metastases and 45 (42\%) had both. The sites of metastases varied and included 95 (89\%) liver, 5 (5\%) non-regional lymph nodes, 5 (5\%) omentum, 1 (1\%) diaphragm, and 1 (1\%) ovary. At initial presentation, patients ranged in age from 31 to 85 years (mean, 60.2; median, 59.0 years) with a slight predominance in male gender (54 of 107, 51\%). The corresponding primary tumors were predominantly located within the pancreatic body and tail $(n=64,60 \%)$ and ranged in size from 1.0 to $18 \mathrm{~cm}$ (mean, $5.3 \mathrm{~cm}$; median, $4.5 \mathrm{~cm}$ ). On the basis of mitotic rate and $\mathrm{Ki}-67$ proliferation index, the primary tumors were classified into the following WHO grades: 29 (27\%) grade 1 (G1), 72 (67\%) grade 2 (G2), and 6 (6\%) grade 3 (G3). In comparison, NF-PanNET metastases were categorized as: 20 (19\%) G1, 79 (74\%) G2 and 8 (7\%) G3. Detailed follow-up information was available for all patients and deaths due to disease were documented for 63 (59\%) patients. Disease-specific survival (DSS) was calculated from the date of primary diagnosis and the date of metastatic diagnosis to the date of death due to disease. DSS rates for this study cohort from the time of primary diagnosis were $95 \%$ at 1 year, $66 \%$ at 5 years and $39 \%$ at 10 years. In comparison, DSS rates for this study cohort from the time of metastasis were $91 \%$ at 1 year, $54 \%$ at 5 years and $32 \%$ at 10 years.

The expression of ARX, PDX1, and both proteins were identified in 71 (66\%), 22 (21\%), and $14(13 \%)$ cases, respectively. Further, loss of ATRX, DAXX and both proteins were detected in $22(21 \%), 34$ (32\%) and 10 (9\%) NF-PanNET metastases. The presence of ALT by telomerespecific FISH was seen in 76 (71\%) of NF-PanNET metastases. Based on ARX, PDX1 and ATRX/DAXX expression, DSS rates from the time of primary diagnosis and from the time of metastasis to death due to disease were not statistically significant $(p>0.05)$.

Primary non-pancreatic neuroendocrine tumors (NETs) were collected from 341 patients and included 48 lung typical/atypical carcinoid tumors, 32 gastric NETs, 41 duodenal NETs, 7 ampullary NETs, 35 jejunal NETs, 123 ileal NETs, 8 colonic NETs, 17 rectal NETs, 28 appendiceal NETs, and 2 gallbladder NETs (Table 3). None of the NETs were clinically functional, and, among 32 gastric NETs, 19 cases were associated with autoimmune metaplastic atrophic gastritis (AMAG). In addition to primary non-pancreatic NETs, 313 nonpancreatic NET metastases were assessed and included 28 cases from the lung (typical/ 
atypical carcinoid), 10 from the stomach, 32 from the duodenum, 71 from the jejunum, 151 from the ileum, 8 from the colon, 10 from the rectum and 3 from the gallbladder. None of the NET metastases were functional and none of the 10 gastric NET metastases arose in the setting of AMAG.

At initial presentation, patients ranged in age from 28 to 87 years (mean, 51.7; median, 55.0 years) with a slight male predominance (347 of 654, 53\%). Among the 341 primary NETs, tumors ranged in size from 0.2 to $7 \mathrm{~cm}$ (mean, $4.7 \mathrm{~cm}$; median, $4.1 \mathrm{~cm}$ ). On the basis of mitotic rate and Ki-67 proliferation index, the 341 primary NETs and 313 NET metastases were classified into the following WHO grades: 510 (78\%) grade 1 (G1), 124 (19\%) grade 2 (G2), and 20 (3\%) grade 3 (G3). Follow-up information was available for 571 of 654 (87\%) patients and ranged from 2 months to 143 months. Among patients with 341 primary NETs, metachronous distant metastases/recurrences were documented for 98 (29\%) patients.

\section{REFERENCES:}

1. Cesare AJ, Heaphy CM, O'Sullivan RJ. Visualization of Telomere Integrity and Function In Vitro and In Vivo Using Immunofluorescence Techniques. Curr Protoc Cytom. 2015;73:12 $401-31$.

2. Singhi AD, Liu TC, Roncaioli JL, Cao D, Zeh HJ, Zureikat $A H$, et al. Alternative Lengthening of Telomeres and Loss of DAXX/ATRX Expression Predicts Metastatic Disease and Poor Survival in Patients with Pancreatic Neuroendocrine Tumors. Clin Cancer Res. 2017;23(2):600-9.

3. Kim JY, Brosnan-Cashman JA, An S, Kim SJ, Song
KB, Kim MS, et al. Alternative Lengthening of Telomeres in Primary Pancreatic Neuroendocrine Tumors Is Associated with Aggressive Clinical Behavior and Poor Survival. Clin Cancer Res. 2017;23(6):1598-606.

4. Hackeng WM, Schelhaas W, Morsink FHM, Heidsma CM, van Eeden S, Valk GD, et al. Alternative Lengthening of Telomeres and Differential Expression of Endocrine Transcription Factors Distinguish Metastatic and Non-metastatic Insulinomas. Endocr Pathol. 2020. 


\section{SUPPLEMENTARY FIGURES}

A

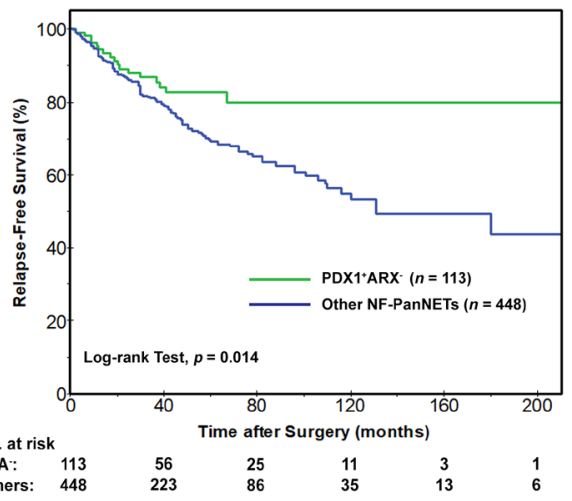

B

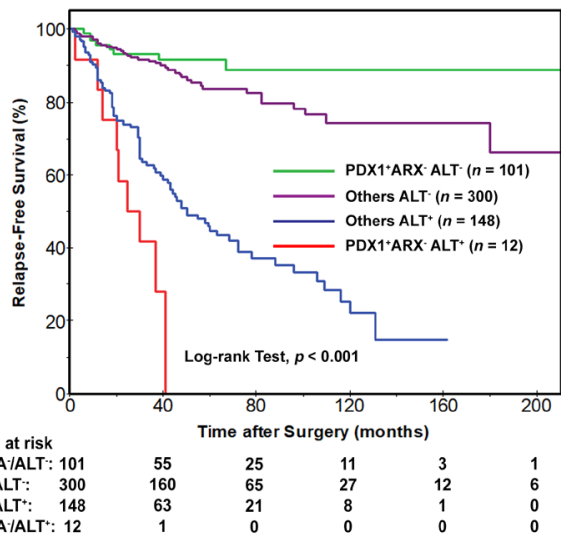

C

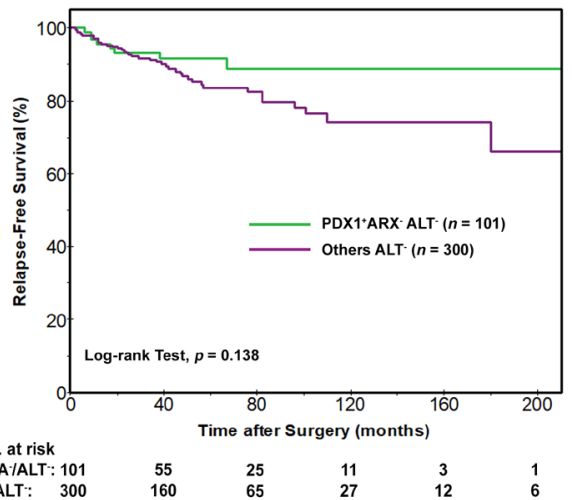

D

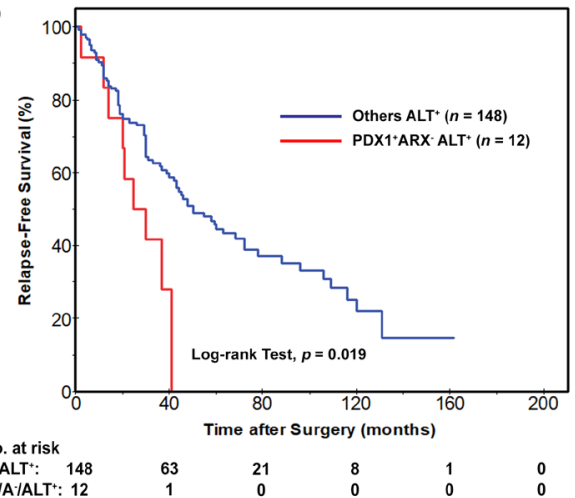

Supplementary Figure 1. Kaplan-Meier curves comparing relapse-free survival (RFS) after surgical resection for patients with PDX1-positive/ARX-negative (PDX1+ARX-) NF-PanNETs versus the remaining cohort. (A) Patients with PDX1+ARX- NF-PanNETs had improved RFS as compared to patients with other NF-PanNETs. However, (B) the prognostic significance between these two cohorts was dependent on ALT status. Among 401 ALT-negative (ALT-) NF-PanNETs, (C) no statistically significant difference in RFS was identified between PDX1+ARX-NF-PanNETs versus the remaining cohort. Interestingly, among 160 ALT-positive (ALT+) NF-PanNETs, (D) a reverse association or shorter RFS was seen for patients with PDX1+ARX-NF-PanNETs as compared to the remaining cohort. This is however likely due to a small number of PDX1+ARX-ALT+ NF-PanNET patients. 

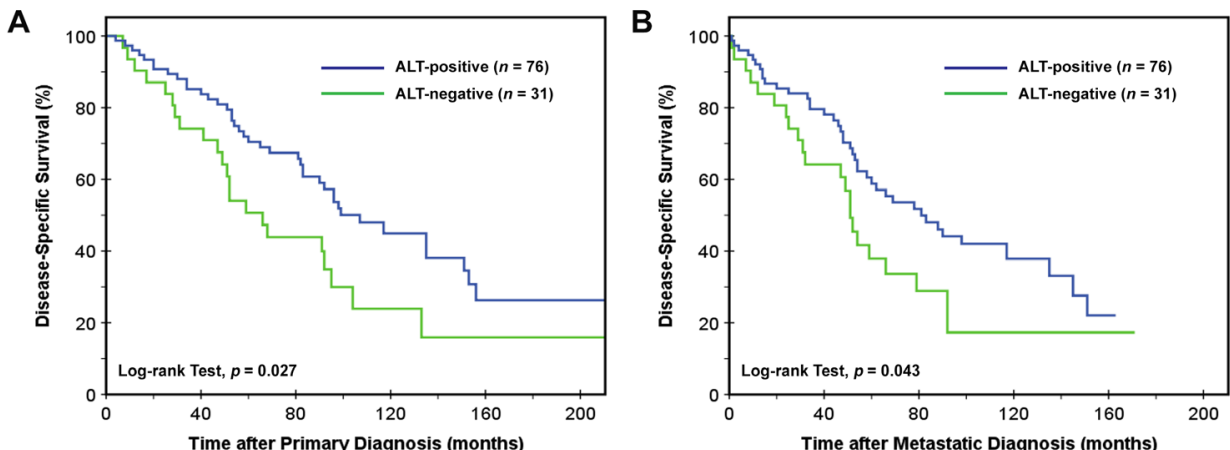

No. at risk

Time after Primary Diagnosis (months)

No. at risk

ALT-pos.: 76
ALT-neg.: 31

$\begin{array}{llcll}60 & 41 & 15 & 5 & 2 \\ 23 & 12 & 4 & 2 & 1\end{array}$

ALT-pos.: 76

Time after Metastatic Diagnosis (months)

\section{Hematoxylin \& Eosin ARX}
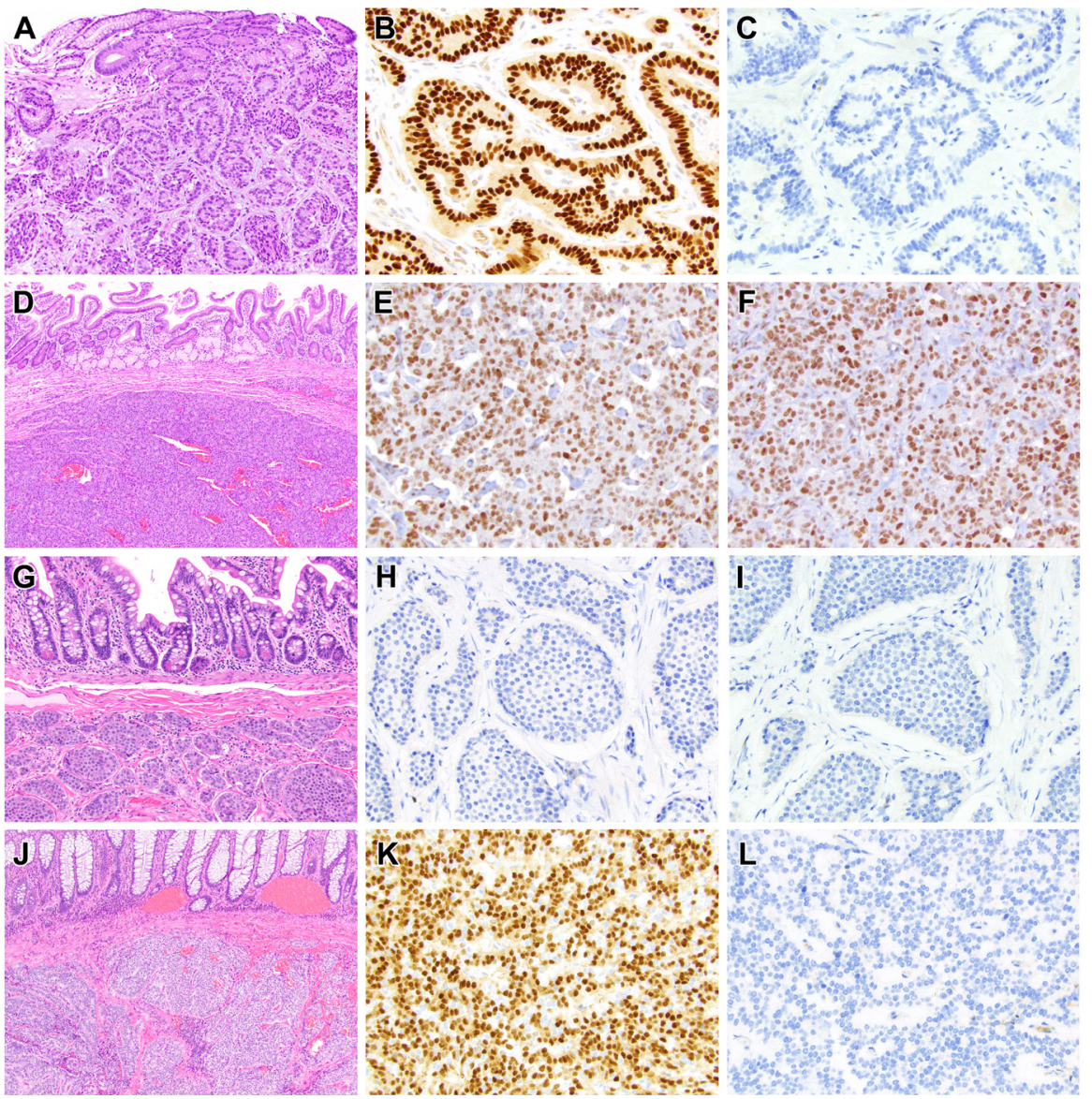
4 page top 142 Supplementary Figure 2. Kaplan-Meier curves of NF-PanNET patients with distant metastases comparing disease-specific survival (DSS) after primary diagnosis (A) and diagnosis of metastatic disease (B). In contrast to patients with a primary NF-PanNET, patients with ALT-positive metastatic NF-PanNETs had longer DSS as compared to patients with ALT-negative metastatic NF-PanNETs.
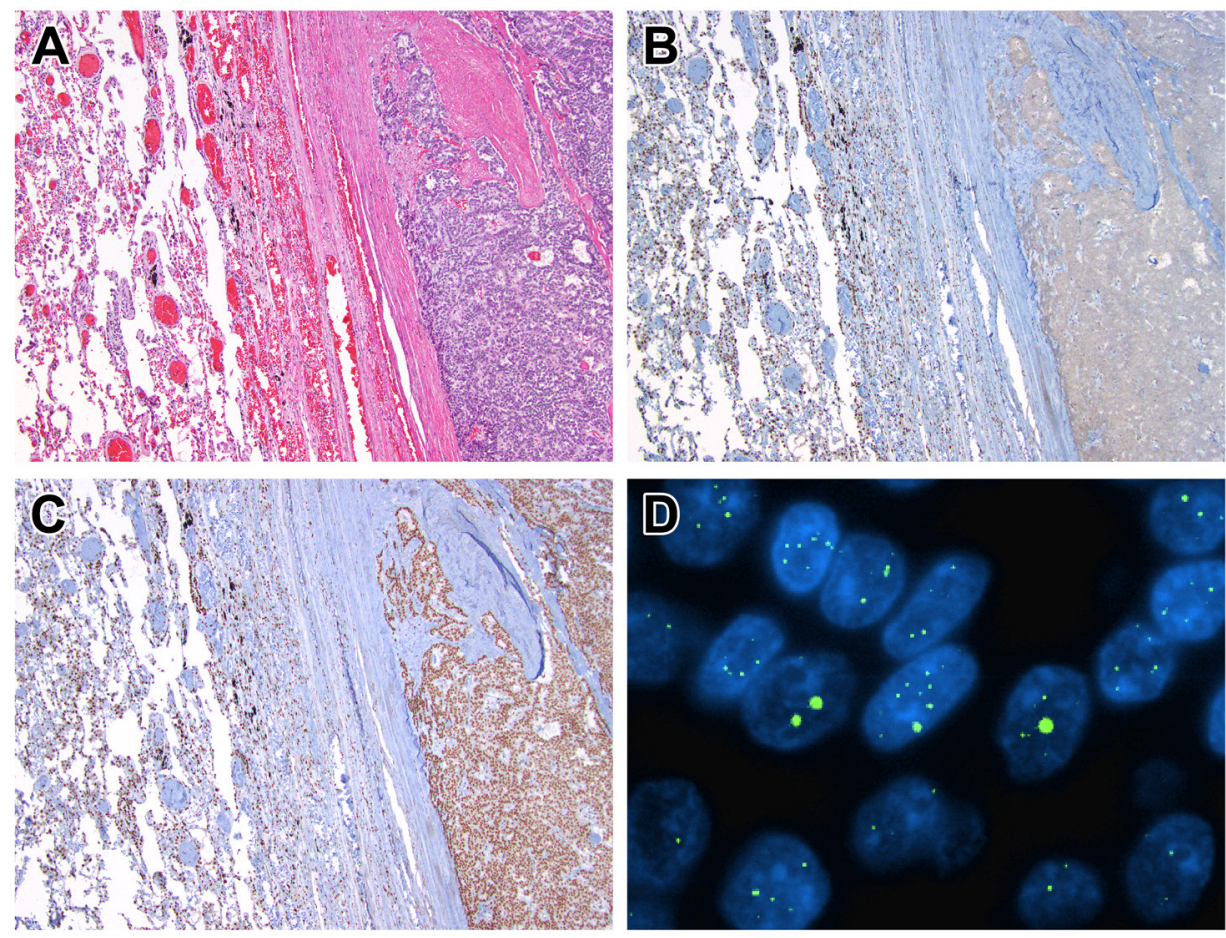

Supplementary Figure 4. Representative example of a (A) lung carcinoid with (B) loss of ATRX nuclear expression and (C) preserved nuclear expression for DAXX. Similar to NF-PanNETs, loss of ATRX and/or DAXX is associated with the presence of large, ultrabright intranuclear foci by telomere-specific FISH, (D) consistent with ALT.

4 Page bottom 142 Supplementary Figure 3. Representative examples of non-pancreatic neuroendocrine tumors (NETs) that were immunolabeled for ARX and PDX1. The majority of gastric NETs (A) were ARX-positive (B) and PDX1-negative (C). Duodenal NETs (D) were more often either ARX-positive/PDX1-positive (E and F) or ARXnegative/PDX1-positive (not shown). In contrast, jejunal and ileal NETs (G) were consistently negative for ARX (H) and PDX1 (I). Finally, rectal NETs (J) were frequently ARX-positive (K) and PDX1-negative (L). 


\section{SUPPLEMENTARY TABLES}

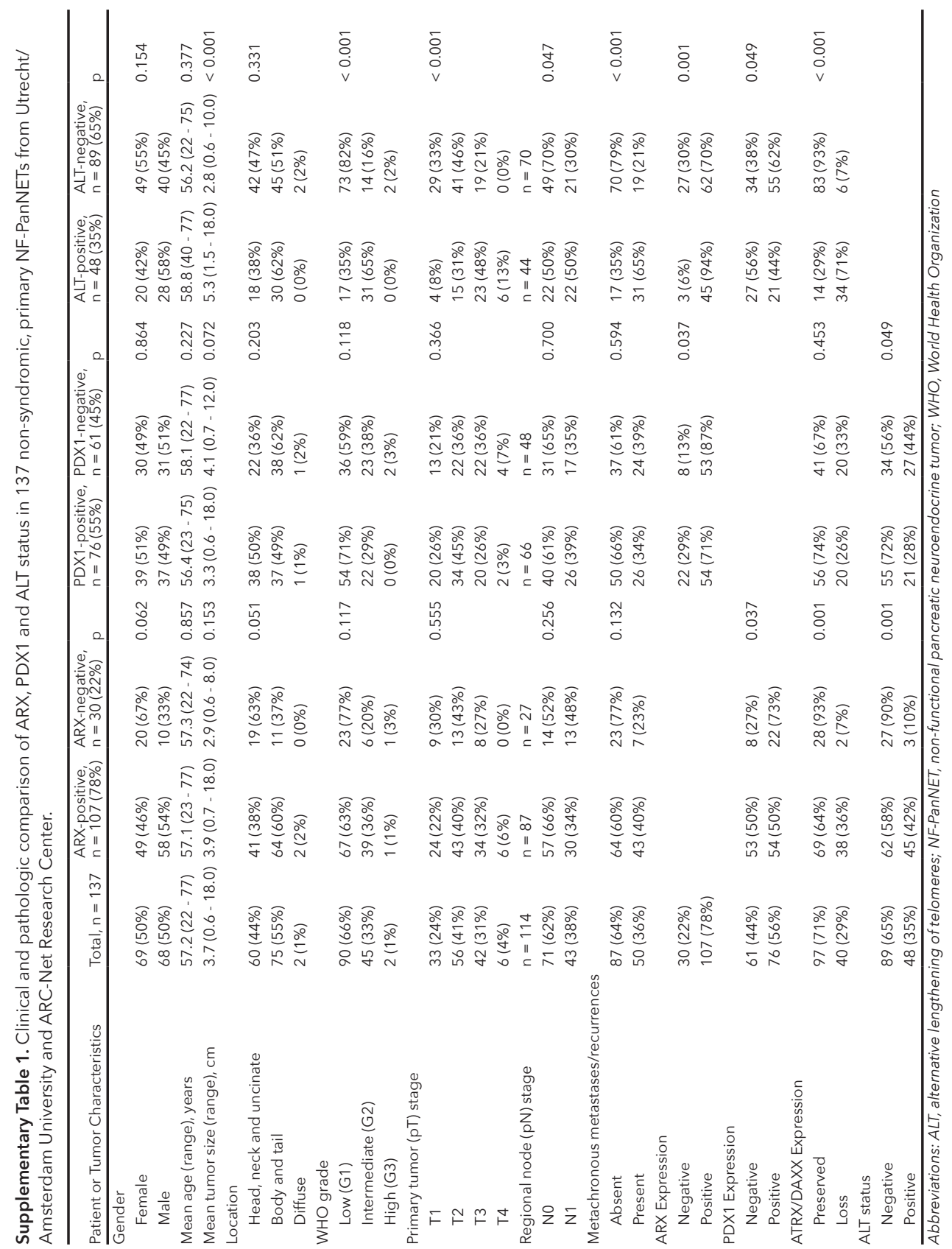




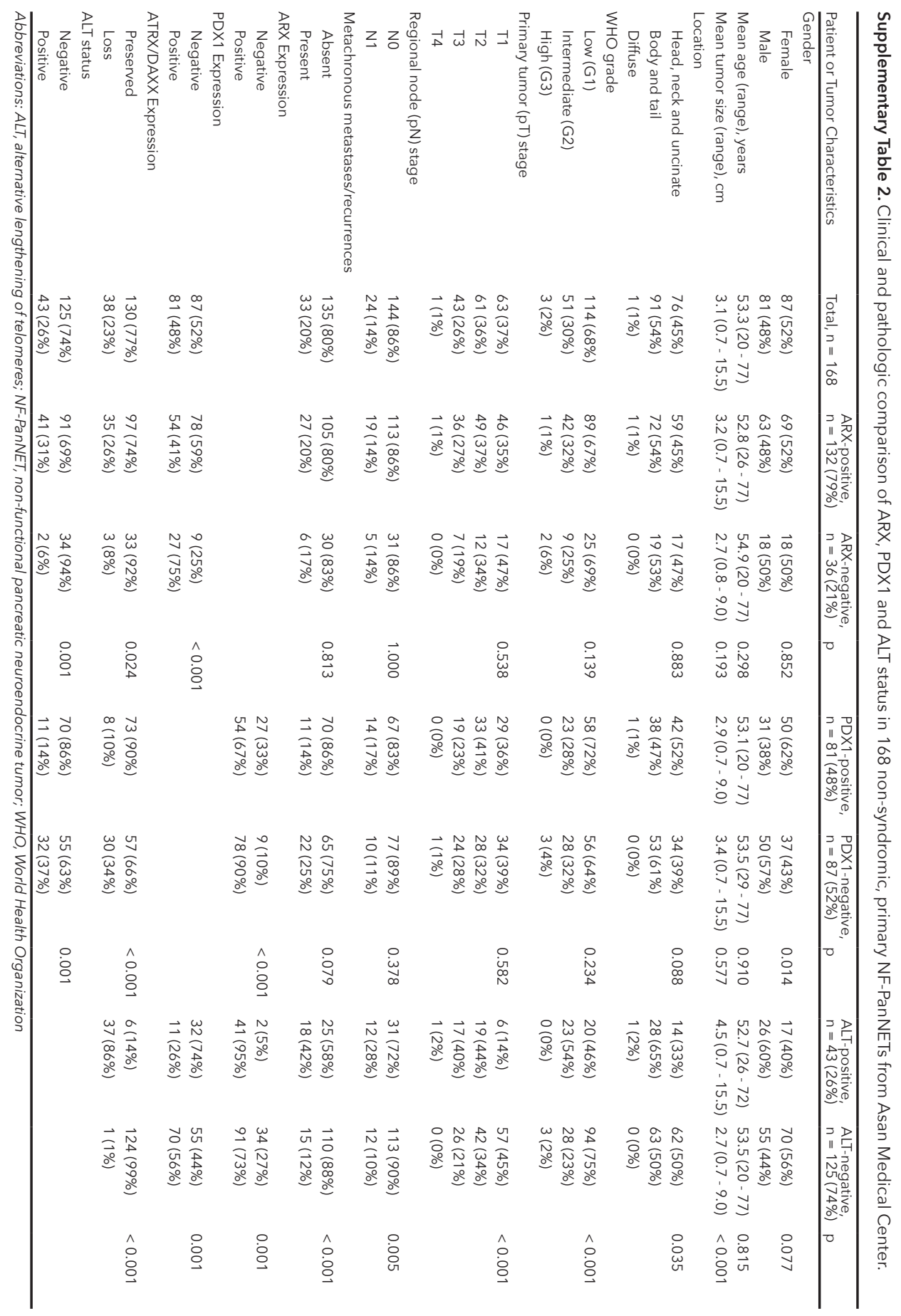




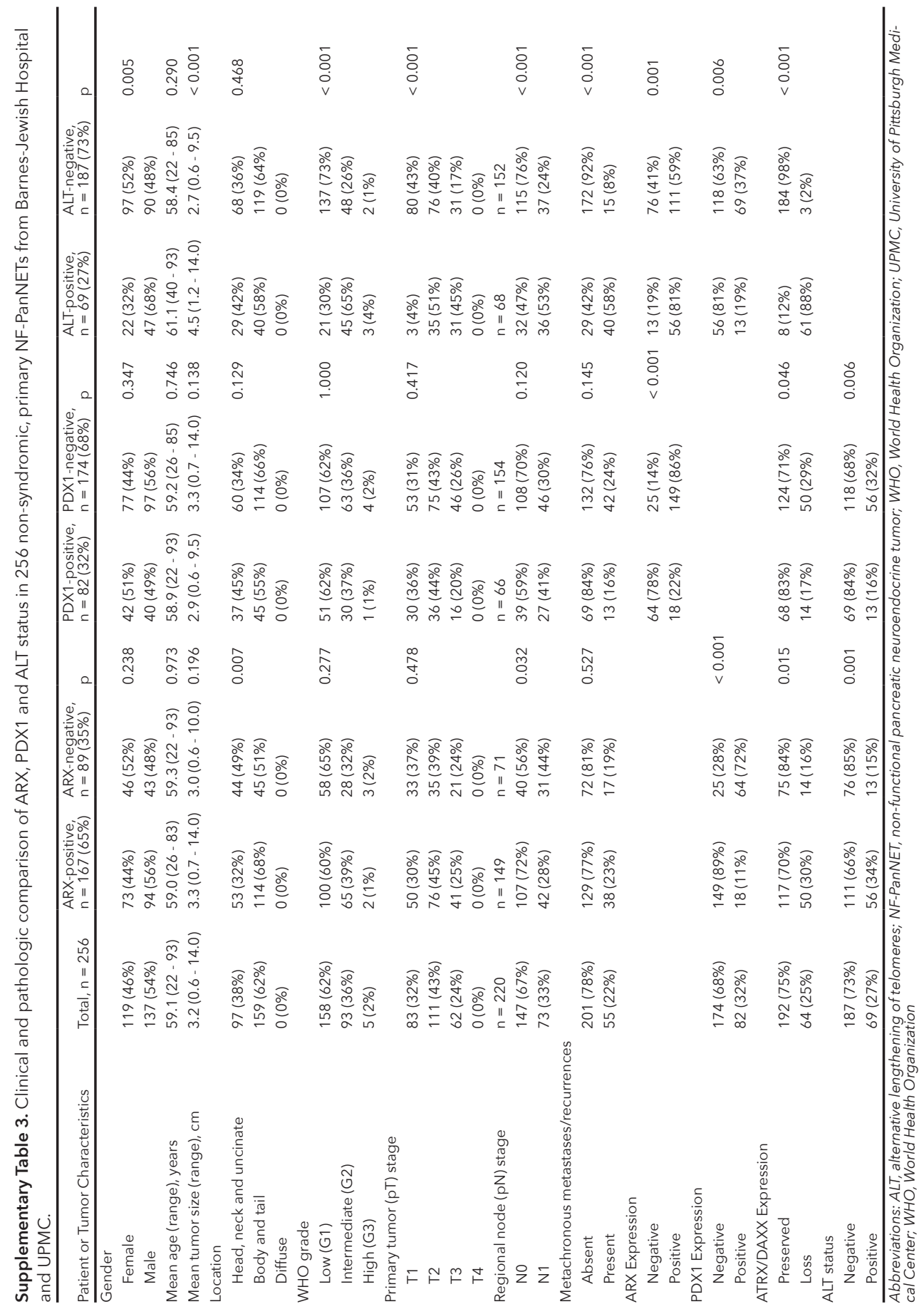


Supplementary Table 4. Clinical and pathologic comparison of 561 primary NF-PanNETs based on the status of ARX and PDX1.

\begin{tabular}{|c|c|c|c|c|c|}
\hline Patient or Tumor Characteristics & $\begin{array}{l}\text { ARX-positive/ } \\
\text { PDX1-negative, } \\
n=280(50 \%)\end{array}$ & $\begin{array}{l}\text { ARX-negative/ } \\
\text { PDX1-positive, } \\
\mathrm{n}=113(20 \%)\end{array}$ & $\begin{array}{l}\text { ARX-positive/ } \\
\text { PDX1-positive } \\
(D P), n=126 \\
(22 \%)\end{array}$ & $\begin{array}{l}\text { ARX-negative/ } \\
\text { PDX1-negative } \\
(D N), n=42 \\
(8 \%)\end{array}$ & $\mathrm{p}$ \\
\hline \multicolumn{6}{|l|}{ Gender } \\
\hline Female & $120(43 \%)$ & $60(53 \%)$ & $71(56 \%)$ & $24(57 \%)$ & 0.031 \\
\hline Male & $160(57 \%)$ & $53(47 \%)$ & $55(44 \%)$ & $18(43 \%)$ & \\
\hline Mean age (range), years & $57.5(26-83)$ & $58.2(20-93)$ & $54.2(23-76)$ & $56.9(22-85)$ & 0.050 \\
\hline Mean tumor size (range), $\mathrm{cm}$ & $3.5(0.7-15.5)$ & $2.8(0.6-9.5)$ & $3.2(0.7-18.0)$ & $3.2(0.7-10.0)$ & 0.049 \\
\hline \multicolumn{6}{|l|}{ Location } \\
\hline Head, neck and uncinate & $91(33 \%)$ & $55(49 \%)$ & $62(49 \%)$ & $25(60 \%)$ & $<0.001$ \\
\hline Body and tail & $188(67 \%)$ & $58(51 \%)$ & $62(49 \%)$ & $17(40 \%)$ & \\
\hline Diffuse & $1(<1 \%)$ & $0(0 \%)$ & $2(2 \%)$ & $0(0 \%)$ & \\
\hline \multicolumn{6}{|l|}{ WHO grade } \\
\hline Low (G1) & $175(63 \%)$ & $82(73 \%)$ & $81(64 \%)$ & $24(57 \%)$ & 0.003 \\
\hline Intermediate (G2) & $101(36 \%)$ & $30(26 \%)$ & $45(36 \%)$ & $13(31 \%)$ & \\
\hline High (G3) & $4(1 \%)$ & $1(1 \%)$ & $0(0 \%)$ & $5(12 \%)$ & \\
\hline Lymphovascular invasion & $n=227$ & $\mathrm{n}=91$ & $\mathrm{n}=72$ & $n=34$ & \\
\hline Absent & $148(65 \%)$ & $60(66 \%)$ & $51(71 \%)$ & $23(68 \%)$ & 0.865 \\
\hline Present & $79(35 \%)$ & $31(34 \%)$ & $21(29 \%)$ & $11(32 \%)$ & \\
\hline Perineural invasion & $n=227$ & $n=91$ & $n=72$ & $n=34$ & \\
\hline Absent & $188(83 \%)$ & $69(76 \%)$ & $60(83 \%)$ & $24(71 \%)$ & 0.208 \\
\hline Present & $39(17 \%)$ & $22(24 \%)$ & $12(17 \%)$ & $10(29 \%)$ & \\
\hline \multicolumn{6}{|l|}{ Primary tumor ( $p T$ ) stage } \\
\hline T1 & $88(31 \%)$ & $47(42 \%)$ & $32(25 \%)$ & $12(29 \%)$ & 0.065 \\
\hline $\mathrm{T} 2$ & $106(38 \%)$ & $41(36 \%)$ & $62(49 \%)$ & $19(45 \%)$ & \\
\hline T3 & $81(29 \%)$ & $25(22 \%)$ & $30(24 \%)$ & $11(26 \%)$ & \\
\hline T4 & $5(2 \%)$ & $0(0 \%)$ & $2(2 \%)$ & $0(0 \%)$ & \\
\hline Regional node (pN) stage & $n=250$ & $\mathrm{n}=95$ & $\mathrm{n}=118$ & $n=39$ & \\
\hline No & $193(77 \%)$ & $62(65 \%)$ & $84(71 \%)$ & $23(59 \%)$ & 0.031 \\
\hline N1 & $57(23 \%)$ & $33(35 \%)$ & $34(29 \%)$ & $16(41 \%)$ & \\
\hline \multicolumn{6}{|c|}{ Metachronous metastases/recurrences } \\
\hline Absent & $205(73 \%)$ & $96(85 \%)$ & $93(74 \%)$ & $29(69 \%)$ & 0.048 \\
\hline Present & $75(27 \%)$ & $17(15 \%)$ & $33(26 \%)$ & $13(31 \%)$ & \\
\hline \multicolumn{6}{|l|}{ ATRX/DAXX Expression } \\
\hline Preserved & $187(67 \%)$ & $101(89 \%)$ & $96(76 \%)$ & $35(83 \%)$ & $<0.001$ \\
\hline Loss & $93(33 \%)$ & $12(11 \%)$ & $30(24 \%)$ & $7(17 \%)$ & \\
\hline \multicolumn{6}{|l|}{ ALT status } \\
\hline Negative & $171(61 \%)$ & $101(89 \%)$ & $93(74 \%)$ & $36(86 \%)$ & $<0.001$ \\
\hline Positive & $109(39 \%)$ & $12(11 \%)$ & $33(26 \%)$ & $6(14 \%)$ & \\
\hline
\end{tabular}

Abbreviations: ALT, alternative lengthening of telomeres; $D N$, double negative for $A R X$ and PDX1; DP, double positive for $A R X$ and PDX1; NF-PanNET, non-functional pancreatic neuroendocrine tumor; WHO, World Health Organization 


\section{Chapter 8}

Supplementary Table 5. Clinical and pathologic comparison of primary NF-PanNETs based on the status of ARX+/ DN, PDX1+/DP and ATRX/DAXX

\begin{tabular}{|c|c|c|c|c|c|c|}
\hline Patient or Tumor Characteristics & $\begin{array}{l}\mathrm{ARX}++\mathrm{DN} \\
\mathrm{n}=322(57 \%)\end{array}$ & $\begin{array}{l}\mathrm{PDX} 1++\mathrm{DP} \\
\mathrm{n}=239(43 \%)\end{array}$ & $\mathrm{p}$ & $\begin{array}{l}\text { Loss of ATRX/ } \\
\text { DAXX } \\
n=142(25 \%)\end{array}$ & $\begin{array}{l}\text { Preserved } \\
\text { ATRX/DAXX, } \\
n=419(75 \%)\end{array}$ & $\mathrm{p}$ \\
\hline \multicolumn{7}{|l|}{$\overline{\text { Gender }}$} \\
\hline Female & $144(45 \%)$ & $131(55 \%)$ & 0.021 & $58(41 \%)$ & $217(52 \%)$ & 0.026 \\
\hline Male & $178(55 \%)$ & $108(45 \%)$ & & $84(59 \%)$ & $202(48 \%)$ & \\
\hline Mean age (range), years & $57.4(22-85)$ & $56.2(20-93)$ & 0.223 & $58.6(26-93)$ & $56.3(20-85)$ & 0.135 \\
\hline Mean tumor size (range), $\mathrm{cm}$ & $3.5(0.7-15.5)$ & $3.0(0.6-18.0)$ & 0.063 & $4.8(0.9-18.0)$ & $2.8(0.6-10.0)$ & $<0.001$ \\
\hline \multicolumn{7}{|l|}{ Location } \\
\hline Head, neck and uncinate & $116(36 \%)$ & $117(49 \%)$ & 0.002 & $50(35 \%)$ & $183(44 \%)$ & 0.133 \\
\hline Body and tail & $205(64 \%)$ & 120 (49\%) & & $91(64 \%)$ & $234(56 \%)$ & \\
\hline Diffuse & $1(<1 \%)$ & $2(1 \%)$ & & $1(1 \%)$ & $2(<1 \%)$ & \\
\hline \multicolumn{7}{|l|}{ WHO grade } \\
\hline Low (G1) & $199(62 \%)$ & $163(68 \%)$ & 0.050 & $55(39 \%)$ & 307 (73\%) & $<0.001$ \\
\hline Intermediate (G2) & $114(35 \%)$ & 75 (32\%) & & $84(59 \%)$ & 105 (25\%) & \\
\hline High (G3) & $9(3 \%)$ & $1(<1 \%)$ & & $3(2 \%)$ & $7(2 \%)$ & \\
\hline Lymphovascular invasion & $\mathrm{n}=261$ & $\mathrm{n}=163$ & & $\mathrm{n}=102$ & $n=322$ & \\
\hline Absent & $171(66 \%)$ & $111(68 \%)$ & 0.599 & $37(36 \%)$ & 245 (76\%) & $<0.001$ \\
\hline Present & $90(34 \%)$ & $52(32 \%)$ & & $65(64 \%)$ & $77(24 \%)$ & \\
\hline Perineural invasion & $n=261$ & $\mathrm{n}=163$ & & $\mathrm{n}=102$ & $n=322$ & \\
\hline Absent & $212(81 \%)$ & $129(79 \%)$ & 0.616 & $66(65 \%)$ & 275 (85\%) & $<0.001$ \\
\hline Present & $49(19 \%)$ & $34(21 \%)$ & & $36(35 \%)$ & $47(15 \%)$ & \\
\hline \multicolumn{7}{|l|}{ Primary tumor (pT) stage } \\
\hline T1 & $100(31 \%)$ & $79(33 \%)$ & 0.404 & $8(6 \%)$ & $171(41 \%)$ & $<0.001$ \\
\hline $\mathrm{T} 2$ & $125(39 \%)$ & $103(43 \%)$ & & $63(44 \%)$ & 165 (39\%) & \\
\hline T3 & $92(28 \%)$ & $55(23 \%)$ & & $67(47 \%)$ & $80(19 \%)$ & \\
\hline $\mathrm{T} 4$ & $5(2 \%)$ & $2(1 \%)$ & & $4(3 \%)$ & $3(1 \%)$ & \\
\hline Regional node ( $\mathrm{pN}$ ) stage & $n=289$ & $n=213$ & & $n=139$ & $n=363$ & \\
\hline NO & $216(75 \%)$ & $146(69 \%)$ & 0.132 & 77 (55\%) & 285 (79\%) & $<0.001$ \\
\hline N1 & $73(25 \%)$ & $67(31 \%)$ & & $62(45 \%)$ & $78(21 \%)$ & \\
\hline \multicolumn{7}{|l|}{$\begin{array}{l}\text { Metachronous distant metastases/ } \\
\text { recurrences }\end{array}$} \\
\hline Absent & $234(74 \%)$ & $189(79 \%)$ & 0.092 & $61(43 \%)$ & $362(86 \%)$ & $<0.001$ \\
\hline Present & $88(26 \%)$ & $50(21 \%)$ & & $81(57 \%)$ & $57(14 \%)$ & \\
\hline \multicolumn{7}{|l|}{ ARX expression } \\
\hline Negative & $42(13 \%)$ & $113(47 \%)$ & $<0.001$ & $19(13 \%)$ & $136(32 \%)$ & $<0.001$ \\
\hline Positive & $280(87 \%)$ & $126(53 \%)$ & & 123 (87\%) & $283(68 \%)$ & \\
\hline \multicolumn{7}{|l|}{ PDX1 expression } \\
\hline Negative & $322(100 \%)$ & $0(0 \%)$ & $<0.001$ & $100(70 \%)$ & $222(53 \%)$ & $<0.001$ \\
\hline Positive & $0(0 \%)$ & $239(100 \%)$ & & $42(30 \%)$ & $197(47 \%)$ & \\
\hline \multicolumn{7}{|l|}{ ATRX/DAXX expression } \\
\hline Preserved & $222(69 \%)$ & $197(82 \%)$ & $<0.001$ & & & \\
\hline Loss & $100(31 \%)$ & $42(18 \%)$ & & & & \\
\hline \multicolumn{7}{|l|}{ ALT status } \\
\hline Negative & 207 (64\%) & $194(81 \%)$ & $<0.001$ & $10(7 \%)$ & $391(93 \%)$ & $<0.001$ \\
\hline Positive & $115(36 \%)$ & 45 (19\%) & & $132(93 \%)$ & $28(7 \%)$ & \\
\hline
\end{tabular}

Abbreviations: ALT, alternative lengthening of telomeres; $D N$, double negative for $A R X$ and PDX 1 ; $P$, double positive for $A R X$ and PDX1; NF-PanNET, non-functional pancreatic neuroendocrine tumor; WHO, World Health Organization 
Supplementary Table 6. Clinical and pathologic comparison of ATRX and DAXX status in 561 non-syndromic, primary NF-PanNETs.

\begin{tabular}{|c|c|c|c|c|c|c|c|}
\hline Patient or Tumor Characteristics & Total, $n=561$ & $\begin{array}{l}\text { Loss of ATRX, } \\
\mathrm{n}=70(12 \%)\end{array}$ & $\begin{array}{l}\text { Preserved } \\
\text { ATRX, } \\
\mathrm{n}=491(88 \%)\end{array}$ & $\mathrm{p}$ & $\begin{array}{l}\text { Loss of DAXX } \\
n=87(16 \%)\end{array}$ & $\begin{array}{l}\text { Preserved } \\
\text { DAXX } \\
n=474(84 \%)\end{array}$ & $\mathrm{p}$ \\
\hline \multicolumn{8}{|l|}{ Gender } \\
\hline Female & 275 (49\%) & $27(39 \%)$ & $248(50 \%)$ & 0.073 & $39(45 \%)$ & $236(50 \%)$ & 0.416 \\
\hline Male & $286(51 \%)$ & $43(61 \%)$ & $243(50 \%)$ & & $48(55 \%)$ & $238(50 \%)$ & \\
\hline Mean age (range), years & $56.9(20-93)$ & $56.8(29-93)$ & $56.9(20-85)$ & 0.604 & $59.7(26-82)$ & $56.4(20-93)$ & 0.025 \\
\hline Mean tumor size (range), $\mathrm{cm}$ & $3.3(0.6-18.0)$ & $4.9(1.1-15.5)$ & $3.0(0.6-18.0)$ & $<0.001$ & $4.7(0.9-18.0)$ & $3.0(0.6-15.5)$ & $<0.001$ \\
\hline \multicolumn{8}{|l|}{ Location } \\
\hline Head, neck and uncinate & $233(41 \%)$ & $28(40 \%)$ & $205(42 \%)$ & 0.864 & $30(35 \%)$ & $203(43 \%)$ & 0.174 \\
\hline Body and tail & $325(58 \%)$ & $42(60 \%)$ & $283(57 \%)$ & & $56(64 \%)$ & $269(56 \%)$ & \\
\hline Diffuse & $3(1 \%)$ & $0(0 \%)$ & $3(1 \%)$ & & $1(1 \%)$ & $2(1 \%)$ & \\
\hline \multicolumn{8}{|l|}{ WHO grade } \\
\hline Low (G1) & $362(65 \%)$ & $21(30 \%)$ & $341(69 \%)$ & $<0.001$ & $38(44 \%)$ & $324(68 \%)$ & $<0.001$ \\
\hline Intermediate (G2) & $189(33 \%)$ & $47(67 \%)$ & $142(29 \%)$ & & 47 (54\%) & $142(30 \%)$ & \\
\hline High (G3) & $10(2 \%)$ & $2(3 \%)$ & $8(2 \%)$ & & $2(2 \%)$ & $8(2 \%)$ & \\
\hline Lymphovascular invasion & $n=424$ & $\mathrm{n}=51$ & $n=373$ & & $n=62$ & $n=362$ & \\
\hline Absent & $282(67 \%)$ & $18(35 \%)$ & $264(71 \%)$ & $<0.001$ & $23(37 \%)$ & $259(72 \%)$ & $<0.001$ \\
\hline Present & 142 (33\%) & $33(65 \%)$ & 109 (29\%) & & $39(63 \%)$ & $103(28 \%)$ & \\
\hline Perineural invasion & $n=424$ & $\mathrm{n}=51$ & $n=373$ & & $n=62$ & $n=362$ & \\
\hline Absent & $341(80 \%)$ & $33(65 \%)$ & $308(83 \%)$ & 0.004 & $41(66 \%)$ & $300(83 \%)$ & 0.005 \\
\hline Present & $83(20 \%)$ & $18(35 \%)$ & $65(17 \%)$ & & $21(34 \%)$ & $62(17 \%)$ & \\
\hline \multicolumn{8}{|l|}{ Primary tumor ( $\mathrm{pT}$ ) stage } \\
\hline $\mathrm{T} 1$ & $179(32 \%)$ & $4(6 \%)$ & $175(36 \%)$ & $<0.001$ & $4(5 \%)$ & $175(37 \%)$ & $<0.001$ \\
\hline $\mathrm{T} 2$ & $228(41 \%)$ & 30 (43\%) & $198(40 \%)$ & & 42 (48\%) & $186(39 \%)$ & \\
\hline T3 & $147(26 \%)$ & $34(48 \%)$ & $113(23 \%)$ & & 39 (45\%) & $108(23 \%)$ & \\
\hline $\mathrm{T} 4$ & $7(1 \%)$ & $2(3 \%)$ & $5(1 \%)$ & & $2(2 \%)$ & $5(1 \%)$ & \\
\hline Regional node $(\mathrm{pN})$ stage & $n=502$ & $n=68$ & $n=434$ & & $n=86$ & $n=416$ & \\
\hline NO & $362(72 \%)$ & $40(59 \%)$ & $322(74 \%)$ & 0.013 & $48(56 \%)$ & $314(76 \%)$ & $<0.001$ \\
\hline N1 & $140(28 \%)$ & $28(41 \%)$ & $112(26 \%)$ & & $38(44 \%)$ & $102(24 \%)$ & \\
\hline \multicolumn{8}{|l|}{$\begin{array}{l}\text { Metachronous metastases/ } \\
\text { recurrences }\end{array}$} \\
\hline Absent & $423(75 \%)$ & $30(43 \%)$ & 393 (80\%) & $<0.001$ & 38 (44\%) & $385(81 \%)$ & $<0.001$ \\
\hline Present & $138(25 \%)$ & 40 (57\%) & $98(20 \%)$ & & 49 (56\%) & 89 (19\%) & \\
\hline \multicolumn{8}{|l|}{ ARX Expression } \\
\hline Negative & 155 (28\%) & $10(14 \%)$ & 145 (30\%) & 0.007 & $12(14 \%)$ & $143(30 \%)$ & 0.002 \\
\hline Positive & $406(72 \%)$ & $60(86 \%)$ & $346(70 \%)$ & & 75 (86\%) & $331(70 \%)$ & \\
\hline \multicolumn{8}{|l|}{ PDX1 Expression } \\
\hline Negative & 322 (57\%) & $53(76 \%)$ & $269(55 \%)$ & 0.001 & 59 (68\%) & $263(56 \%)$ & 0.034 \\
\hline Positive & $239(43 \%)$ & $17(24 \%)$ & $222(45 \%)$ & & $28(32 \%)$ & $211(44 \%)$ & \\
\hline \multicolumn{8}{|l|}{ ATRX Expression } \\
\hline Preserved & $491(88 \%)$ & & & & $73(84 \%)$ & $418(88 \%)$ & 0.289 \\
\hline Loss & $70(12 \%)$ & & & & $14(16 \%)$ & $56(12 \%)$ & \\
\hline \multicolumn{8}{|l|}{ DAXX Expression } \\
\hline Preserved & $474(84 \%)$ & $56(80 \%)$ & $418(85 \%)$ & 0.289 & & & \\
\hline Loss & $87(16 \%)$ & $14(20 \%)$ & $73(15 \%)$ & & & & \\
\hline \multicolumn{8}{|l|}{ ALT status } \\
\hline Negative & $401(71 \%)$ & $4(6 \%)$ & $397(81 \%)$ & $<0.001$ & $7(8 \%)$ & $394(83 \%)$ & $<0.001$ \\
\hline Positive & $160(29 \%)$ & $66(94 \%)$ & $97(19 \%)$ & & $80(92 \%)$ & $80(17 \%)$ & \\
\hline
\end{tabular}

Abbreviations: ALT, alternative lengthening of telomeres; NF-PanNET, non-functional pancreatic neuroendocrine tumor; WHO, World Health Organization 


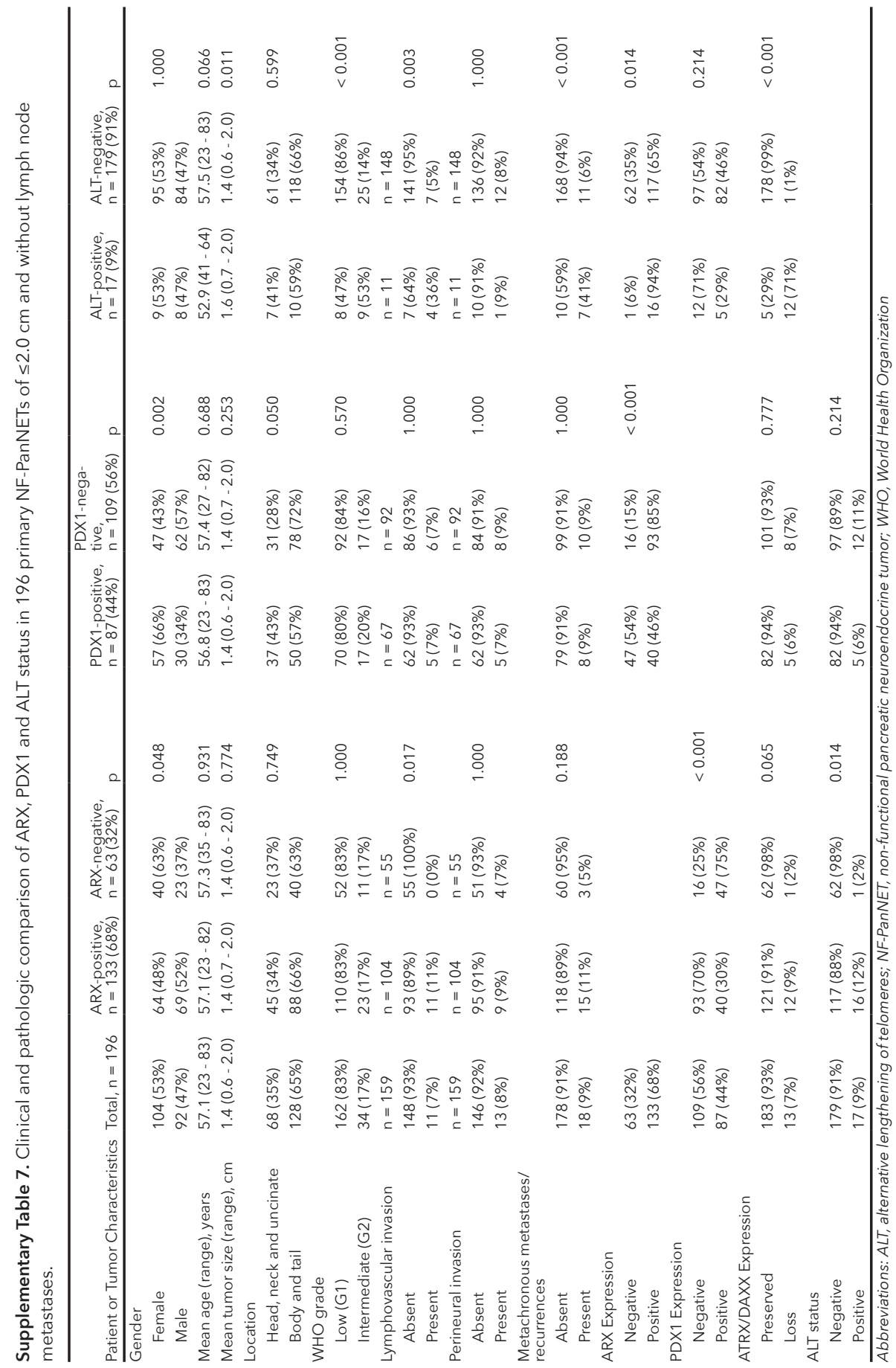


Supplementary Table 8. RFS rates based on tumor size thresholds for NF-PanNETs of $\leq 2.0 \mathrm{~cm}$ and without lymph node metastases.

\begin{tabular}{|c|c|c|c|}
\hline Tumor size threshold & $\begin{array}{l}1,5 \text { and } 10 \text {-year RFS rates, } \\
\text { below and equal to }(\leq) \text { tumor size threshold }\end{array}$ & $\begin{array}{l}1,5 \text { and } 10 \text {-year RFS rates, } \\
\text { above }(>) \text { tumor size threshold }\end{array}$ & $\mathrm{p}$ \\
\hline $0.7 \mathrm{~cm}$ & $92 \%, 92 \%$ and $92 \%$ & $97 \%, 89 \%$ and $78 \%$ & 0.883 \\
\hline $0.8 \mathrm{~cm}$ & $95 \%, 95 \%$ and $95 \%$ & $97 \%, 89 \%$ and $78 \%$ & 0.529 \\
\hline $0.9 \mathrm{~cm}$ & $96 \%, 96 \%$ and $96 \%$ & $97 \%, 89 \%$ and $77 \%$ & 0.394 \\
\hline $1.0 \mathrm{~cm}$ & $98 \%, 98 \%$ and $98 \%$ & $96 \%, 87 \%$ and $75 \%$ & 0.099 \\
\hline $1.1 \mathrm{~cm}$ & $98 \%, 94 \%$ and $94 \%$ & $96 \%, 88 \%$ and $75 \%$ & 0.150 \\
\hline $1.2 \mathrm{~cm}$ & $99 \%, 95 \%$ and $95 \%$ & $96 \%, 87 \%$ and $74 \%$ & 0.059 \\
\hline $1.3 \mathrm{~cm}$ & $97 \%, 95 \%$ and $95 \%$ & $96 \%, 86 \%$ and $72 \%$ & 0.054 \\
\hline $1.4 \mathrm{~cm}$ & $97 \%, 95 \%$ and $95 \%$ & $96 \%, 86 \%$ and $72 \%$ & 0.042 \\
\hline $1.5 \mathrm{~cm}$ & $97 \%, 91 \%$ and $91 \%$ & $96 \%, 86 \%$ and $65 \%$ & 0.046 \\
\hline $1.6 \mathrm{~cm}$ & $98 \%, 92 \%$ and $92 \%$ & $95 \%, 85 \%$ and $61 \%$ & 0.017 \\
\hline $1.7 \mathrm{~cm}$ & $97 \%, 90 \%$ and $90 \%$ & $96 \%, 88 \%$ and $58 \%$ & 0.040 \\
\hline $1.8 \mathrm{~cm}$ & $97 \%, 89 \%$ and $89 \%$ & $97 \%, 92 \%$ and $58 \%$ & 0.095 \\
\hline $1.9 \mathrm{~cm}$ & $97 \%, 89 \%$ and $89 \%$ & $97 \%, 91 \%$ and $58 \%$ & 0.074 \\
\hline
\end{tabular}

Abbreviations: N/A, not available; NF-PanNET, non-functional pancreatic neuroendocrine tumor; RFS, relapse-free survival 


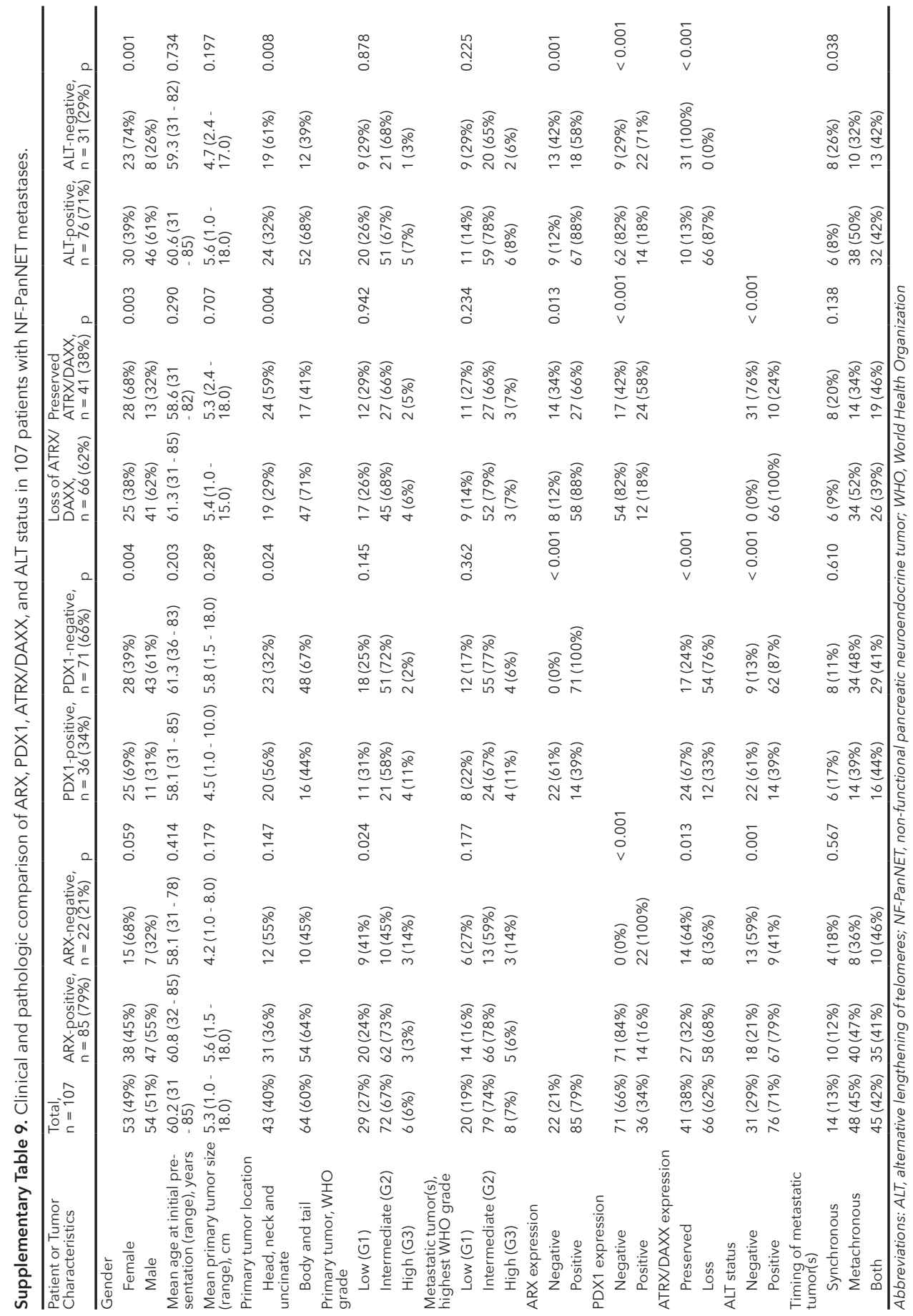




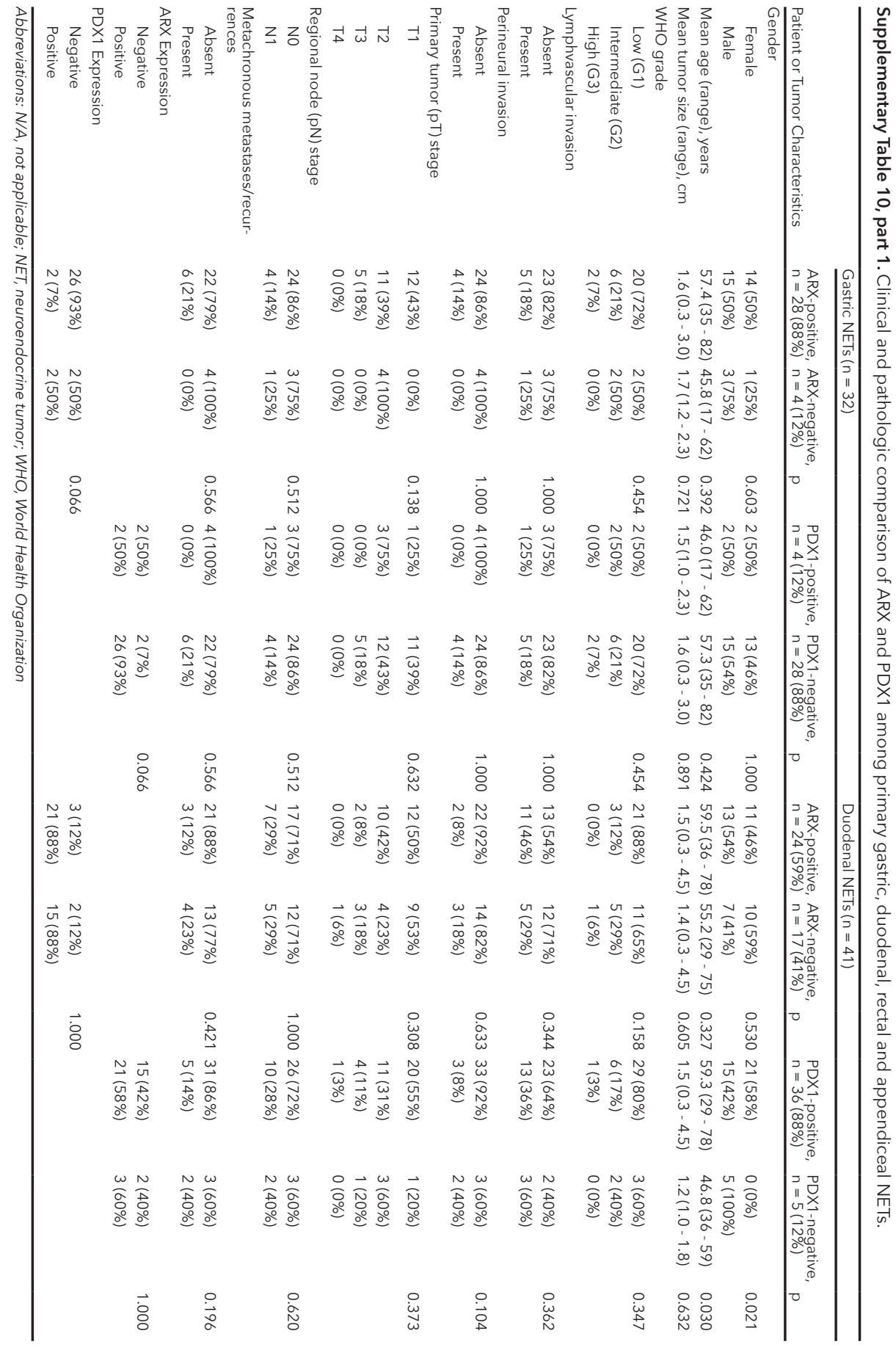




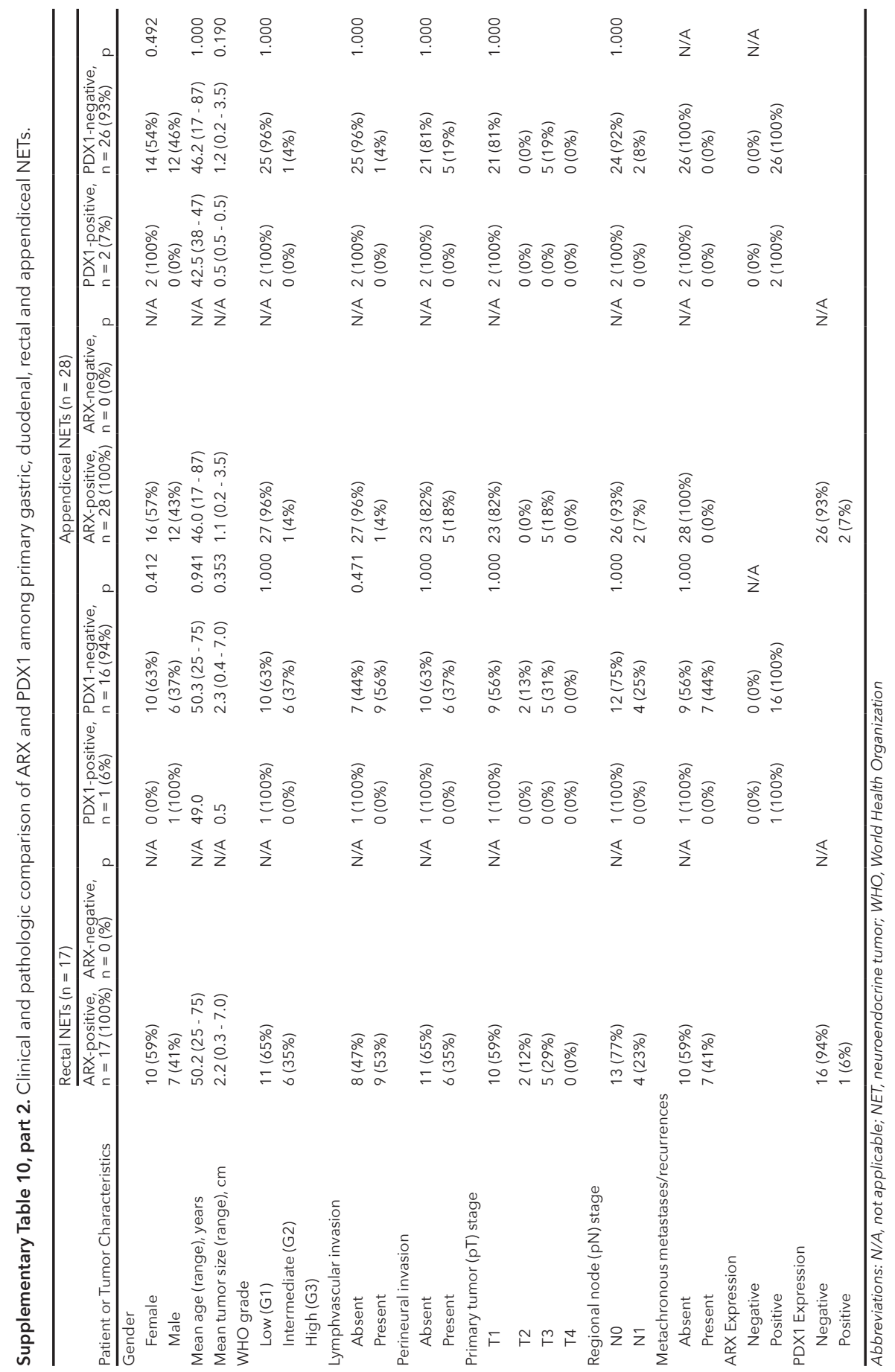



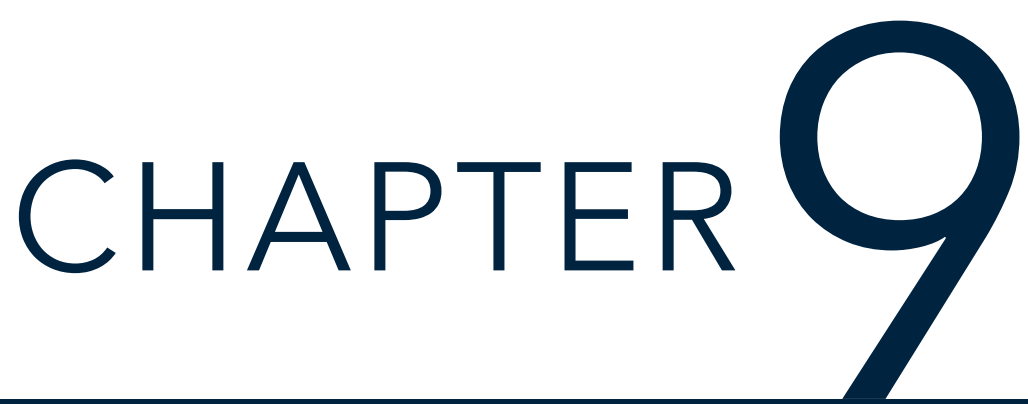

Metastatic patterns of duodenopancreatic neuroendocrine tumors in patients with multiple endocrine neoplasia type 1 van Treijen, Inne H.M. Borel Rinkes, Koen M.A. Dreijerink, G. Johan A. Offerhaus, Gerlof D. Valk, Menno R. Vriens, Lodewijk A.A. Brosens 


\section{ABSTRACT}

Patients with multiple endocrine neoplasia 1 syndrome (MEN1) often develop multifocal duodenopancreatic neuroendocrine tumors (NET). Non-functional pancreatic NETs and duodenal gastrinomas are the most frequent origins of metastasis. Current guidelines recommend surgery based on tumor functionality, size $\geq 2 \mathrm{~cm}$, grade or presence of lymph node metastases. However, in case of multiple primary tumors it is often unknown which specific tumor metastasized. This study aims to unravel the relationship between primary duodenopancreatic NETs and metastases in patients with MEN1 by studying endocrine differentiation.

First, it was shown that expression of the endocrine differentiation markers ARX and PDX1 was concordant in 18 unifocal sporadic NETs and matched metastases. Thereafter, ARX, PDX1, Ki67 and gastrin expression, and the presence of alternative lengthening of telomeres (ALT) were determined in 137 microscopic and macroscopic duodenopancreatic NETs and 36 matched metastases in 10 patients with MEN1. ARX and PDX1 $\mathrm{H}$-score clustering was performed to infer relatedness. For patients with multiple metastases, similar intra-metastases transcription factor expression suggests that most metastases (29/32) originated from a single NET of origin, while few patients may have multiple metastatic primary NETs. In six patients with MEN1 and hypergastrinemia, peri-duodenopancreatic lymph node metastases expressed gastrin, and clustered with minute duodenal gastrinomas, not with larger pancreatic NETs. Pancreatic NET metastases often clustered with high grade or ALT-positive primary tumors.

In conclusion, for patients with MEN1-related hypergastrinemia and PanNETs, a duodenal origin of peri-duodenopancreatic lymph node metastasis should be considered, even when current conventional and functional imaging studies don't reveal duodenal tumors preoperatively. 


\section{INTRODUCTION}

Metastatic duodenopancreatic neuroendocrine tumors (dpNETs) are the main cause of death in patients with multiple endocrine neoplasia type 1 syndrome (MEN1) (1). The syndrome is caused by germline mutations in the tumor suppressor gene MEN1 and is characterized by the occurrence of parathyroid adenomas, pituitary tumors, pancreatic NETs (PanNETs) - insulinomas or non-functional PanNETs - and duodenal gastrinomas. For MEN1-associated PanNETs, operative resection is the cornerstone of curative therapy and guidelines recommend surgery based on size ( $\geq 2 \mathrm{~cm}$ ), growth rate, hormone production and radiological signs suspicious of malignancy like lymph node metastases (LNM) (2-4). Indications for surgical resection of MEN1-associated gastrinoma are more controversial, but include size and the presence of LNM (2-4).

The high diagnostic accuracy of 68Gallium-labelled somatostatin analog positron emission tomography has increased the detection rate of LNM and distant metastases in patients with MEN1 (5). As it is often unclear whether locoregional LNM originate from PanNETs or gastrinomas, choosing the surgical procedure is challenging, especially since major duodenopancreatic resections in patients with MEN1 are associated with severe complications and a remarkable cumulative burden of morbidity $(6,7)$.

Histopathological duodenopancreatic resection specimens in patients with MEN1 typically reveal multiple PanNETs, many microadenomas (PanNETs $<0.5 \mathrm{~cm}$ ) and minute duodenal gastrinomas. For this reason, also the postoperative determination of the primary origin of metastases is challenging, which hampers the appropriate use and applicability of tumor, node, and metastasis (TNM) staging systems, limiting prognostication of individual patients. Better understanding of the origins of metastases may help to refine the criteria for surgery and tailor duodenopancreatic resections to the individual patient's disease.

Recently, histone modification patterns and DNA methylation profiles revealed that PanNETs come in a range of distinct endocrine-cell differentiations, which show similarities to normal endocrine alpha and beta cells (8-11). Transcription factors ARX and PDX1, which are surrogate immunohistochemical markers for endocrine differentiation (9), are expressed in similar proportions of primary non-functional PanNETs and a separate cohort of distant PanNET metastases (12). Although yet to be confirmed in matched primary PanNETs and metastases, this suggests that endocrine differentiation is preserved in metastases. 
Therefore, we questioned if transcription factor expression could serve to match locoregional and distant metastases to a specific primary tumor in patients with multifocal MEN1-associated dpNETs. Furthermore, in case of multiple (locoregional) metastases, it is unknown if multiple primary tumors of origin should be considered. An in-depth understanding of the metastatic patterns of MEN1-associated dpNETs is key to justify major duodenopancreatic surgery.

Here, we aim to unravel the relatedness of multifocal primary dpNETs and metastases in patients with MEN1. First, the preservation of immunohistochemical ARX and PDX1 expression in 18 sporadic unifocal NETs and 50 matched metastases was confirmed. Thereafter, a detailed analysis was performed to assess the relation between multifocal primary dpNETs and microadenomas $(n=137)$ and matched metastases $(n=36)$ in 10 patients with MEN1.

\section{MATERIALS AND METHODS}

\section{Selection}

The use of archival material was approved by the University Medical Center Utrecht (UMCU) Biobank Research Ethics Committee. The UMCU pathology archives were searched for NETs within duodenopancreatic resection specimens from 1990-2017. Autopsies were excluded. The identified specimens were cross referenced with the Dutch MEN1 database from the DutchMEN Study Group (DMSG) to identify patients with MEN1 (13). Mutation negative patients fulfilling clinical MEN1 criteria were excluded. Patients with sporadic disease were eligible for further investigation if formalin-fixed, paraffin-embedded material (FFPE) was available of a unifocal primary PanNET and at least one metastasis. Patients with MEN1 were eligible for further investigation if FFPE material was reported of at least two dpNETs or microadenomas and at least one metastasis. Metastases were defined as tumor positive lymph nodes or distant metastases, at any time during follow-up.

\section{Data collection}

Pathological data were collected from the pathology reports (tumor size, lymph node involvementand distant metastases) and clinical data were obtained from the DMSG database (13). Clinical data included demographic data (age and sex), laboratory values (fasting serum gastrin (FSG) levels), preoperative conventional (computed tomography, magnetic resonance imaging or endoscopic ultrasonography) or functional imaging (68Gallium- 
labelled somatostatin analog positron emission tomography) and multidisciplinary tumor board discussion. Tumor size was determined on conventional imaging as previously described (14). The presence and number of duodenal NETs, PanNETs in the pancreatic head or body/tail, and presence of LNM was based on conventional and functional imaging derived up to six months before surgery. Gastroduodenoscopies were evaluated for lesions suspicious for duodenal NETs.

Histologic slides were reviewed and PanNETs (i.e., $\geq 0.5 \mathrm{~cm}$ ), pancreatic microadenomas (i.e., $<0.5 \mathrm{~cm}$ ), duodenal NETs and metastases were annotated. All sections containing dpNETs or metastases were selected for further analysis. In addition, several slides containing only pancreatic microadenomas were included. Macroscopic gross tumor size was extracted from the pathology reports if available, and was otherwise measured on the microscopic slides. Peri-duodenopancreatic LNM were defined as LNM within pancreatoduodenectomy, but not distal pancreatectomy resection specimens, e.g., pancreaticoduodenal and infrapyloric lymph node stations.

\section{Immunohistochemistry and telomere fluorescence in situ hybridization}

$4 \mu \mathrm{m}$ sections of the selected tissue blocks were cut, cleared at $60^{\circ} \mathrm{C}$ and deparaffinized. Immunohistochemistry was performed on consecutive sections as previously described (11) with antibodies against ARX (1:2000, MABN102, Millipore, Burlington, MA) and PDX1 (1:2000, ab134150, Abcam, Cambridge, United Kingdom). Immunohistochemistry for Gastrin and Ki67 was similarly performed with the following conditions: 20 minutes antigen retrieval in $10 \mathrm{mM}$ citrate $(\mathrm{pH} 6)$ at $100^{\circ} \mathrm{C}, 1$-hour incubation of gastrin (1:3000, A0568, Agilent, Santa Clara, CA) and Ki67 (1:100, SP6, Thermo Fisher, Waltham MA) antibodies with DAB or bright-DAB as chromogen (Immunologic, VWRKBS04-110), respectively. Immunohistochemistry for ATRX (1:100, HPA0001906, Sigma, St. Louis, MO) and DAXX (1:400, HPA008736, Atlas antibodies, Bromma, Sweden) was performed only for cases with ultrabright telomeric foci determined by telomere fluorescence in situ hybridization (FISH) (11). Telomere FISH was performed as previously described (11) using telomere specific Cy3 labeled probes (100 nM, F1002 Lot no. 180723PL-01, Panagene, Yuseong-gu, Republic of Korea) and centromere specific probes (100 nM, F3013 Lot no. 172865, Panagene) as hybridization control.

\section{Scoring}

ARX, PDX1, and gastrin expression were scored in consensus by two observers (LAAB, 
WMH, ME, ASMK). ARX and PDX1 percentages of nuclear staining were scored on a continuous scale, and intensities were scored as absent, weak, intermediate or strong. Before consensus scoring, ARX and PDX1 stained sporadic tumors were scored independently $(\mathrm{LAAB}, \mathrm{WMH})$ for categorical percentages of staining cells $(0 \%,<10 \%,<50 \%,<70 \%$, $<100 \%$ ) and intensities for the determination of interobserver agreement (11). If patchy expression was present, a representative average was determined reflecting the whole slide. Dichotomous expression of transcription factors was determined using previously described cut-offs for expression (11). An estimated H-score (range 0-300) for ARX and PDX1 was calculated per lesion by multiplying representative staining nuclear intensity (0-3) and continuous percentage (0-100). Ki67 nuclear expression was digitally scored in the two highest labeling tumor areas, in 2000 cells if present, using a previously validated digital counting method on scanned slides (15). Tumor grade was assigned following the World Health Organization (WHO) 2017 classification (Grade 1 (G1) <3\%, G2 3-20\%, G3 $>20 \%$ Ki67 labeling-index) (16). Cytoplasmic gastrin expression was scored as absent or present. Alternative lengthening of telomeres (ALT) was defined as $>1 \%$ of cells containing ultrabright telomeric signals $>10 x$ the cumulative signal intensity of all telomeric signals in a normal stromal cell. The percentage of ultrabright telomeric signals was visually assessed at 20x magnification (WMH), foci were confirmed at 100x magnification, and signal intensities were quantified using Telometer as previously described (11).

\section{Statistical analysis}

Non-normally distributed continuous variables were presented as median (interquartile range $[I Q R]$ or range). The Mann-Whitney $U$ test was used to assess differences in continuous variables between two groups. The Chi-squared test was used for comparisons of categorical variables. Two-sided p-values $<0.05$ were considered as statistically significant. The Euclidean distance was used to calculate similarities between intra- and inter-patient lesions and for hierarchical clustering. In interobserver analysis, the weighted kappa with equal spacing was calculated as measure of reliability, and interpreted as previously described (17). Data transformations, statistical tests, and data visualization were performed using R version 3.6.3 (R Foundation for Statistical Computing, Vienna, Austria).

\section{RESULTS}

\section{ARX and PDX1 expression and ALT are preserved in sporadic PanNET metastases}

To establish the preservation of immunohistochemical ARX and PDX1 expression as surrogate clonal markers of differentiation, we identified 18 patients with sporadic unifocal 


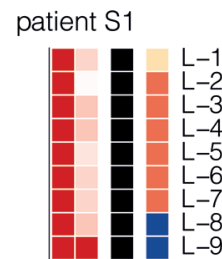

patient S2

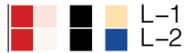

patient S3

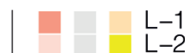

patient S4

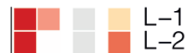

patient S5

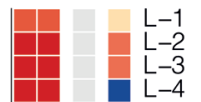

patient S6

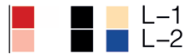

patient S7

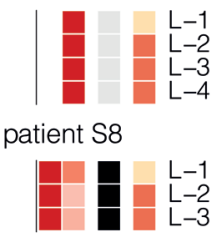

patient S9

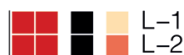

patient S10

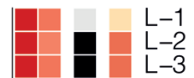

patient S11

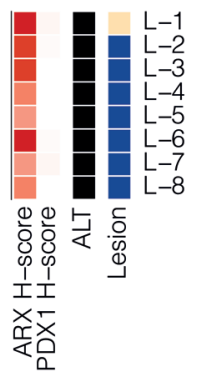

patient S12

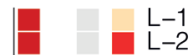

patient S13

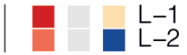

patient S14

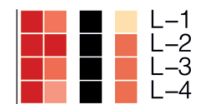

patient S15

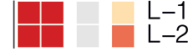

patient $\mathrm{S} 16$

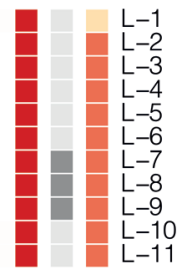

patient S17

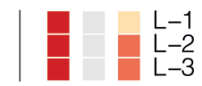

patient S18
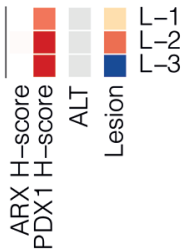

H-score

strongest expression

$$
\text { no expression }
$$

ALT

Normal Telomeres

ALT

$\mathrm{NI}$

\section{Lesion}

Bone metastasis

Adrenal metastasis

Liver metastasis

Lymph node metastasis

Pancreas primary 
primary PanNETs and 50 matched LNM or distant metastases. The cohort consisted of 8 females and 10 males with a median age of 59 years (range 42-76). The median primary tumor size measured $5.0 \mathrm{~cm}$ (IQR 2.7-9.0). 12 PanNETs were non-functional and 6 were functional (2 insulinomas, 2 glucagonomas, 1 gastrinoma and 1 VIPoma).

Analysis of the Euclidean distance between $\mathrm{H}$-scores of the primary and matched metastatic tumors revealed close intra-patient proximity (median distance 20, IQR 3-65). Indeed, visual inspection of $\mathrm{H}$-scores also showed a highly similar ARX and PDX1 expression between primary tumors and matched metastases (Figure 1). Notably, in matched primary tumors and metastases, ARX expression was either consistently present or absent/near absent. However, for PDX1, two metastases showed a change in expression (patients no. S1 and S4), whereas the vast majority of metastases (48/50) showed a similar pattern to primary tumors and/or other metastases. Transcription factors were scored highly similar between two observers, substantiated by almost perfect reliability of ARX scoring (percentage kappa 0.94, intensity kappa 0.89), and almost perfect and substantial reliability of PDX1 scoring (percentage kappa 0.86, intensity kappa 0.74).

In the same cohort, telomere FISH showed that ALT was clonally present in 7 patients, in which both primary tumors and all 23 matched metastases were ALT positive. In one additional patient (patient no. S10), the primary tumor was ALT negative, while 2 metastases were ALT positive. This high degree of similarity indicates that ARX and PDX1 expression profiles and ALT are preserved during progression, and thus can be used as surrogate markers for clonal relatedness between primary tumors and metastases.

\section{MEN1 cohort}

Within our institution, 27 patients with genetically proven MEN1 underwent duodenopancreatic resections of whom 20 had multifocal dpNETs with or without metastasis (flowchart in Supplementary Figure 1). Ten patients with FFPE tissue specimens of multiple dpNETs and metastasis were eligible for inclusion: 6 had locoregional LNM during the primary resection, and 4 patients had lymph node, retroperitoneal, liver or lung metastases removed during other procedures. Of note, the lung metastases showed TTF1

Page 162 Figure 1. Sporadic PanNET metastases. Consensus ARX and PDX1 H-scores (immunohistochemical nuclear staining intensity ${ }^{\star}$ percentage of staining cells) and alternative lengthening of telomere (ALT) status of 18 patients with sporadic unifocal primary PanNETs and synchronous or metachronous metastases. In most patients, highly similar patterns can be seen for ARX and PDX1 expression and ALT for matched primary tumors and lymph node or distant metastases. Abbreviation: NI not interpretable. 
expression at initial histopathological analysis, suggesting primary pulmonary NET. Of the ten patients, $50 \%$ were males, median age at surgery was 50 years (range $31-63$ ), and surgical indications included non-functional PanNET $(n=2)$, insulinoma $(n=2)$, gastrinoma $(n=3)$, and non-functional PanNET plus gastrinoma $(n=3)$ (Table 1, Supplementary Table 1$)$. $\operatorname{Most}(n=9)$ had radiological evidence of multiple PanNETs. The median radiological size of the largest PanNET or microadenoma measured $2 \mathrm{~cm}$ (range 0.3-4.8). Duodenal NETs were reported preoperatively in 5 out of 6 patients with suspected gastrinoma: conventional imaging was positive in 3 out of 6, gastroduodenoscopy in 3 out of 4 and PET/CT in 3 out of 5 , respectively.

\section{Histopathological characteristics of MEN1-associated dpNETs}

In total, 32 PanNETs, 88 pancreatic microadenomas $(<0.5 \mathrm{~cm})$, and 17 duodenal NETs and 36 metastases were scored for ARX and PDX1 expression (Table 2, Figure 2, Supplementary Figure 2). Two patients did not have multifocal dpNETs or distant metastases after extensive histopathological analysis. In patient no. M4, the resected lung lesions were negative for ARX and PDX1 expression and positive for TTF1 and therefore considered primary pulmonary NETs (12). In patient no. M6, a microadenoma that was initially observed was not present in deeper sections, and only a single PanNET (the largest) remained. These two patients were partially included in further analyses: patient no. M4 for the descriptive statistics of primary tumor transcription factor expression, and patient no. M6 for the analysis of relatedness between primary tumors and metastases.

Median size measured $1.04 \mathrm{~cm}$ (range 0.50-4.50) for PanNETs, $0.10 \mathrm{~cm}$ (range 0.05-0.40) for microadenomas and $0.10 \mathrm{~cm}$ (range 0.05-1.00) for duodenal NETs. In four patients (patients no. M3, M8-M10), the largest duodenal tumor was $<0.5 \mathrm{~cm}$. Sole PDX1 expression (ARX-/ PDX1+) was exclusively seen in PanNETs (Table 2). Both pancreatic and duodenal tumors showed cases with sole ARX (ARX+/PDX1-) or double positive expression (ARX+/PDX1+). Compared to PanNETs, microadenomas were significantly more often positive for ARX only (ARX+/PDX1-). No transcription factor subtype was specifically associated with metastases (Table 2). Examples of the immunohistochemical staining are shown in Figure 3. ALT was only seen in a single patient (patient no. M7).

\footnotetext{
Page 165 Figure 2. Metastastic patterns of dpNETs in patients with MEN1. Metastatic patterns in patients no. M1, M3, M5, M8, M9, and M10. Unsupervised hierarchical clustering is performed on the ARX and PDX1 H-score heatmap. An $\mathrm{H}$-score is the immunohistochemical nuclear staining intensity multiplied by the percentage of staining cells. Lesions are annotated with size (except metastases), Ki67 labeling index, gastrin expression, the presence of alternative lengthening of telomeres (ALT), and anatomic location.
} 


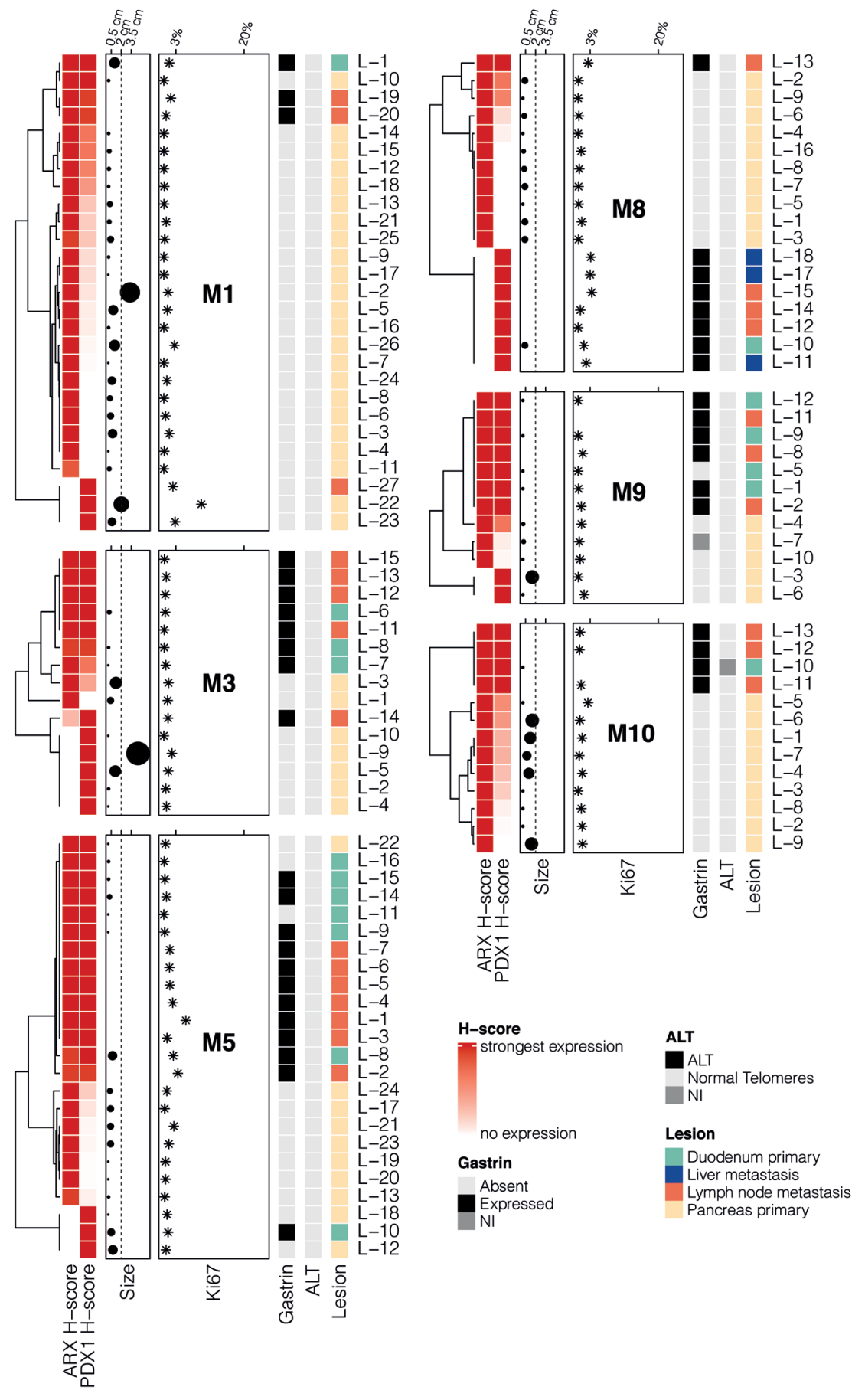




\section{Metastatic patterns of MEN1-associated dpNETs}

Intra-patient Euclidean distance hierarchical clustering on the ARX and PDX1 H-scores was performed to discover relations between primary tumors and metastases. Metastases within individual patients clustered together and showed close proximity to each other (Figure 2). In line, the transcription factor proximity between multiple intra-patient (i.e., in patients with multiple metastases) metastases in MEN1 $(n=32)$ was similar to the distance observed between matched metastases in the sporadic setting (median Euclidean distance 0 [IQR 0-42] versus 3, [IQR 0-41], respectively, p=0.07; Supplementary Figure 3). 3 out of 32 metastases (in patients no. M1, M3, M8) clustered apart and had a different transcription factor profile compared to other intra-patient metastases. This indicates that in the presence of multiple metastases, the majority of the metastases likely derived from one single primary tumor, but synchronous metastatic events may occur. In contrast, no relatedness was observed between different primary tumors in patients with MEN1, and a significantly greater Euclidean distance was seen for intra-patient primaries as compared to intra-patient metastases (median 145 [IQR 30-297] versus 0 [IQR 0-42], respectively, $\mathrm{p}<0.001$; Supplementary Figure 3). In every patient, one or more primary tumors clustered with the metastases, identifying them as possible primary tumor of origin.

\section{Origin of duodenopancreatic lymph node metastases with suspected gastrinoma}

Six patients (patients no. M1, M3, M5, M8-M10) underwent a pancreatoduodenectomy or total pancreatectomy and had LNM (Figure 2). All peri-duodenopancreatic LNMs clustered tightly to duodenal NETs and few pancreatic microadenomas, but not to the (larger) PanNETs. Of note, patient no. M1 (pancreatoduodenectomy plus distal pancreatectomy) also had a single LNM (L-27) located around the pancreatic tail which clustered with two PanNETs in the tail (Figure 2, Figure 3). All duodenal NETs and most associated LNM were WHO grade 1 (G1). In two patients with suspected gastrinoma (patients no. M5 and M8), a Ki67 labeling index of $\geq 3 \%$ (G2) was seen in LNMs. To rule out the presence of (microscopic) pancreatic gastrinoma, and subsequently confirm the suspected clonal relations based on ARX and PDX1 expression, we performed gastrin immunohistochemistry. Indeed, not one PanNET or microadenoma, but most primary duodenal NETs (14/17), and all peri-duodenopancreatic LNM expressed gastrin. In two patients with gastrin positive lymph nodes, a single gastrin positive LNM (patient no. M3 L-14 and patient no. M8 L-13) showed aberrant ARX expression compared to other LNM, while all other metastases showed relatively uniform ARX and PDX1 expression. Furthermore, these two aberrantly staining LNM did not cluster with any dpNET, but as complete duodenal embedding was not performed, it is possible 


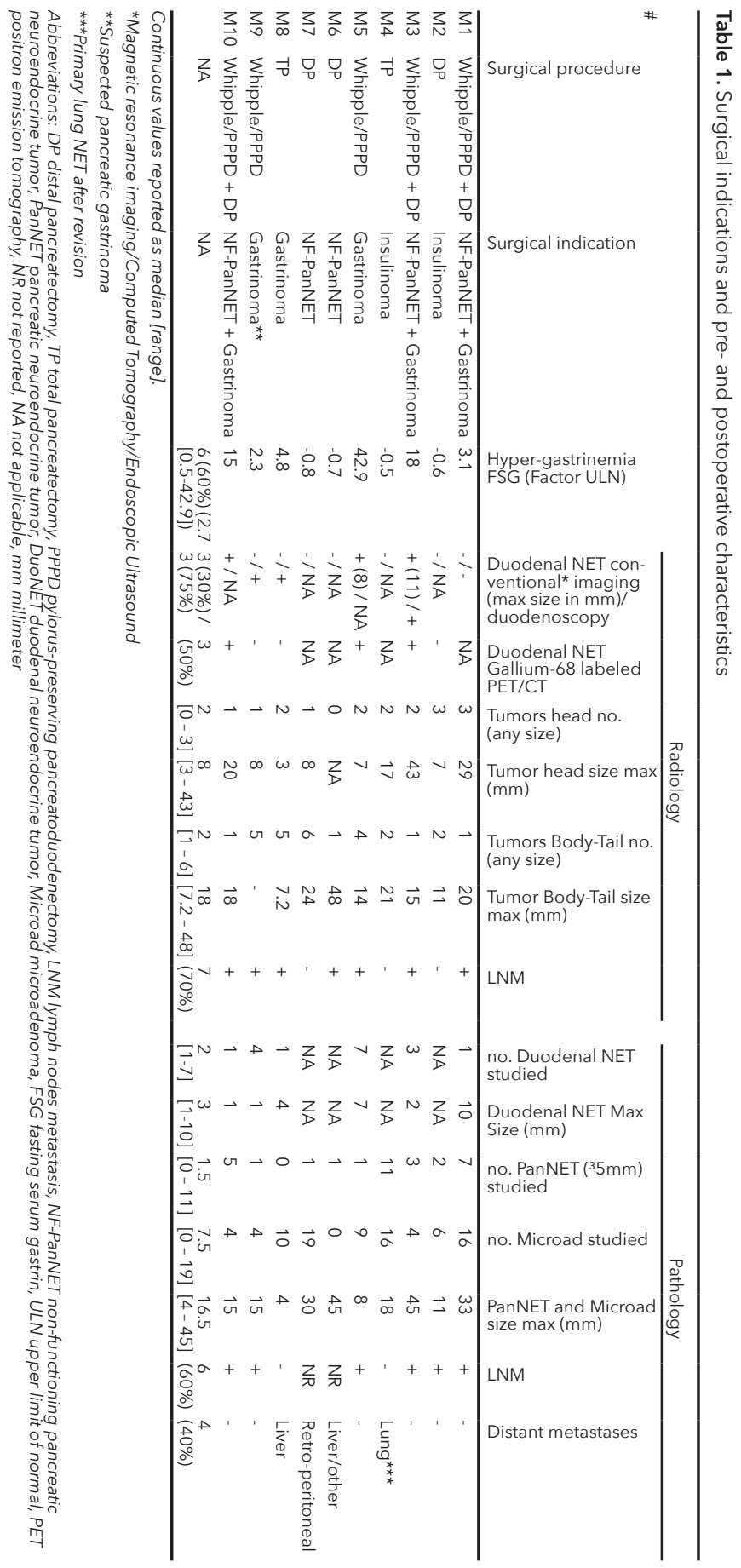




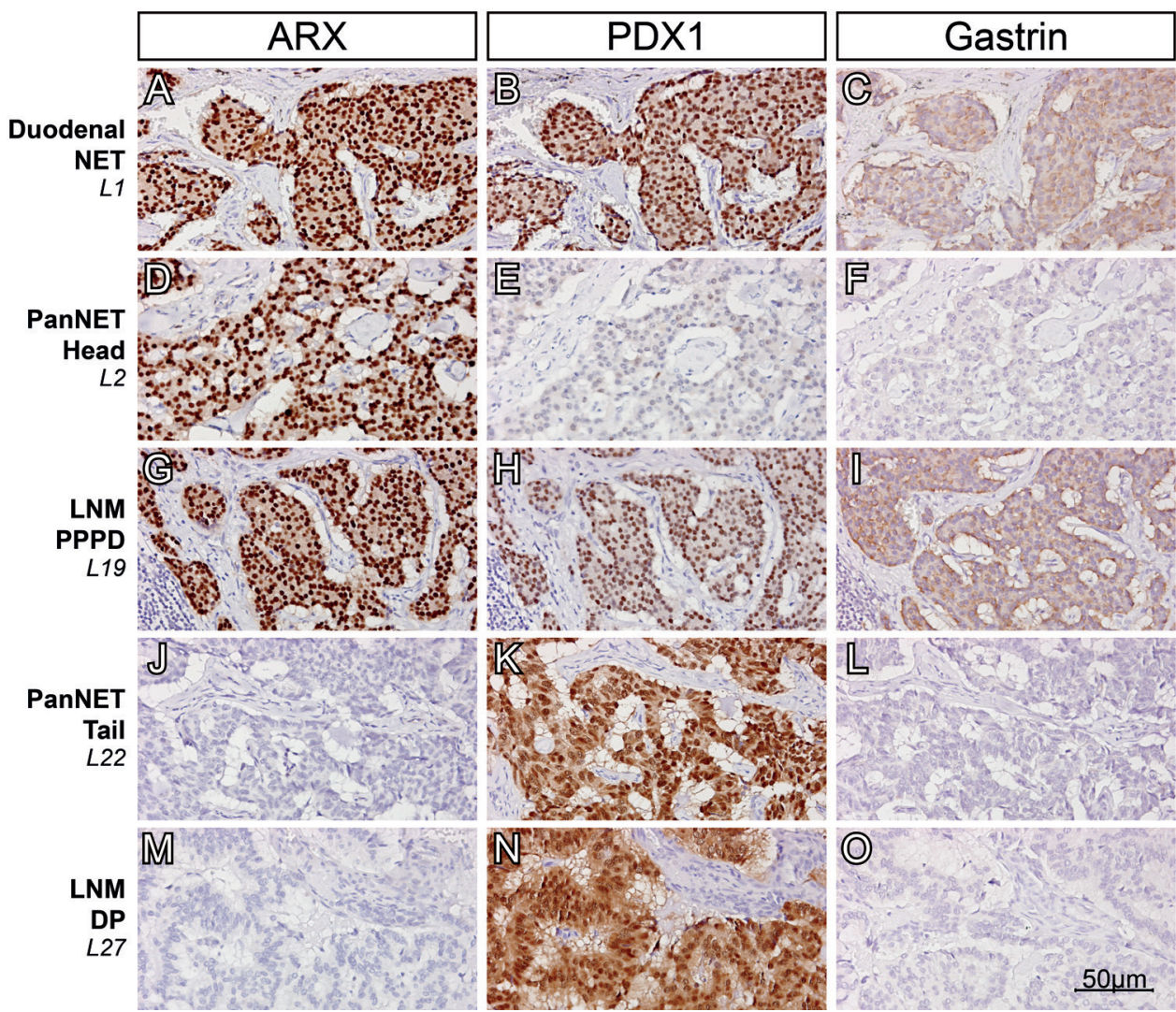

Figure 3. ARX, PDX1, gastrin immunohistochemistry in single patient with MEN1. Representative images of $A R X$ and PDX1 and Gastrin expression from patient no. M1, with L-numbers linking to Figure 2. A-C, lesion L-1, the largest primary duodenum NET $(1 \mathrm{~cm})$. D-F, lesion L-2, the largest primary PanNET $(3.3 \mathrm{~cm})$ in the pancreatic head. G-I, lesion $\mathrm{L}-19$, lymph node metastases in pylorus-preserving pancreatoduodenectomy specimen. J-L, lesion L-22, primary PanNET $(2 \mathrm{~cm})$ in the pancreatic tail. M-O, lesion L-27, lymph node metastases in distal pancreatectomy specimen. Abbreviations: NET neuroendocrine tumor, PanNET pancreatic neuroendocrine tumor, LNM lymph node metastasis, PPPD pylorus-preserving pancreaticoduodenectomy, DP distal pancreatectomy.

Table 2. Transcription factors, grade and ALT in primary dpNETs and metastases

\begin{tabular}{|c|c|c|c|c|c|c|c|c|}
\hline & ARX+/PDX1- & ARX-/PDX1+ & ARX+/PDX1+ & ARX-/PDX1- & $\mathrm{WHO}=\mathrm{G} 1$ & $\mathrm{WHO}>\mathrm{G} 1$ & ALT+ & Total \\
\hline $\begin{array}{l}\text { Pancreatic mi- } \\
\text { croadenomas }\end{array}$ & $48 / 88(55 \%)$ & $18 / 88(20 \%)$ & $22 / 88(25 \%)$ & - & $87 / 88(99 \%)$ & $1 / 88(1 \%)$ & $1 / 88(1 \%)$ & 88 \\
\hline $\begin{array}{l}\text { Pancreatic } \\
\text { NETs }{ }^{\star}\end{array}$ & $9 / 32(28 \%)$ & $12 / 32(38 \%)$ & $11 / 32(34 \%)$ & - & $26 / 32(81 \%)$ & $6 / 32(19 \%)$ & $1 / 32(3 \%)$ & 32 \\
\hline Duodenal NETs & $0 / 17(0 \%)$ & $2 / 17(12 \%)$ & $15 / 17(88 \%)$ & - & $16 / 16^{\star \star}(100 \%)$ & $0 / 16^{\star \star}(0 \%)$ & $0 / 16^{\star \star}(0 \%)$ & 17 \\
\hline $\begin{array}{l}\text { Gastrin + meta- } \\
\text { stases }\end{array}$ & $0 / 27(0 \%)$ & $6 / 27(22 \%)$ & $21 / 27(78 \%)$ & - & $21 / 26$ ** $(81 \%)$ & $5 / 26^{* *}(19 \%)$ & $0 / 27(0 \%)$ & 27 \\
\hline $\begin{array}{l}\text { Gastrin - meta- } \\
\text { stases }\end{array}$ & 4 & 1 & 2 & - & 2 & 5 & 1 & 7 \\
\hline Other ${ }^{\star \star \star}$ & - & - & - & 2 & 2 & - & - & 2 \\
\hline
\end{tabular}


that several microscopic primary tumors were not embedded as FFPE block (18).

\section{Origin of metastases without suspected gastrinoma}

In four patients (patients no. M1, M2, M6, M7) metastases clustered with primary PanNETs (Figure 2, Supplementary Figure 2). In patient no. M1, a G1 LNM showed clustering with two primary PanNETs, located in the distal pancreatectomy specimen (L-22, 2cm, G2 and L-23, $0.6 \mathrm{~cm}, \mathrm{G} 1)$. In patient no. M2, the LNM (G1) did not cluster with the largest PanNET (L-8, $1.1 \mathrm{~cm}, \mathrm{G} 2)$, but instead showed greater proximity to a smaller PanNET (L-1, 0.9cm, G1) and two microadenomas. Of note, the original pathology report noted insulin expression in L-8, while L- 1 and the LNM were insulin negative. Patient 6 and 7 both had a $\geq 2 \mathrm{~cm}$ G2 primary PanNET with G2/G3 metastases. In patient 7 the primary tumor (L-21) and retroperitoneal metastasis (L-1), and a $1 \mathrm{~mm}$ microadenoma were ALT positive (Supplementary Figure 4). All three ALT positive lesions had retained ATRX and DAXX expression.

\section{DISCUSSION}

In patients with MEN1, the expected oncological benefits of operative resection and the anticipated risk of complications must be weighed for every single dpNET. However, predicting tumor behavior is challenging in the preoperative setting. In this histopathological study, we inferred relations of primary dpNETs, locoregional and distant metastases by ARX and PDX1 transcription factor clustering in 10 patients with MEN1 and in 18 patients with sporadic PanNETs. In patients with MEN1 and hypergastrinemia, none of the peri-duodenopancreatic LNM had a pancreatic origin. Instead, most showed similar transcription factor expression to duodenal gastrinomas and all expressed gastrin. In patients with multiple metastases, intra-metastases transcription factor similarity indicated that most metastases originated from a single dpNET. One of the exceptions included a patient with two metastasized tumors; a gastrinoma with peri-duodenopancreatic LNM and a PanNET within the pancreatic tail with a local peripancreatic LNM.

This study provides detailed insight in the metastatic patterns of MEN1-associated dpNETs, in particular for patients with hypergastrinemia and radiological LNM. In six patients with MEN1 and hypergastrinemia, all 27 peri-duodenopancreatic LNM - and not a single PanNET - expressed gastrin. Previous histopathological studies have found similar associations. Pipeleers-Marichal et al. initially reported eight patients with MEN1 and hypergastrinemia, of which four had peri-duodenopancreatic LNM (19). All peri-duodenopancreatic LNM expressed gastrin, as did all 29 primary duodenal NETs. In contrast, none of 12 PanNETs, 
and only one out of 115 microadenomas expressed gastrin. Anlauf et al. reported 18 patients with MEN1 and hypergastrinemia, including the 8 previously reported patients by Pipeleers-Marichal et al. In 10 patients with LNM, all LNM were gastrin positive (20). Most patients (15/18) had primary duodenal gastrinomas and none had pancreatic gastrinomas. These data indicate that gastrinomas in MEN1 have a duodenal origin and are associated with gastrin expressing locoregional lymph node metastases. In the present series, we report a detailed analysis for all individual LNM. In addition to gastrin, virtually all peri-duodenopancreatic LNM (25/27) showed similar ARX and PDX1 transcription factor expression to the duodenal gastrinomas and not a single LNM showed a similar expression pattern to a PanNET. This is important novel evidence of relatedness, as it has been described that hormonal production of metastases can change as compared to the primary NET $(19,21)$. Furthermore, two individual gastrin positive LNM showed different transcription factor expression compared to other gastrin positive metastases of the same patients. This suggests multiclonal LNM, which has been reported in MEN1 before (22). The present study provides firm evidence for a common duodenal origin of peri-duodenopancreatic LNM in patients with MEN1 and hypergastrinemia, which will contribute to more accurate, and tumor-specific staging in case of multifocal duodenopancreatic disease in MEN1. Furthermore, as four out of six patients with hypergastrinemia had primary gastrinomas $<0.5 \mathrm{~cm}$, a duodenal origin of peri-duodenopancreatic LNM should even be considered, even if no duodenal NETs can be found on imaging or during gastroduodenoscopy.

Management of gastrinomas in MEN1 is challenging for endocrinologists and surgeons. The widespread use of proton pump inhibitors (PPI) has reversed the hypersecretion state and its consequences, and has shifted the therapeutic aim to preventing metastasis-related morbidity and mortality. The benefit of major surgery for MEN1-associated gastrinoma is controversial, because of the variation in diagnosis, treatment strategies, and prognosis presented in different studies. A universal consensus regarding surgical indication, timing, type of procedure and extent of surgery is lacking (23).

In the first place, several studies have shown that the prognosis of majority patients with MEN1 and gastrinomas is excellent if PanNETs $<2 \mathrm{~cm}(3,24)$, regardless of therapy or the presence of peri-duodenopancreatic LNM (25). Although a recent large multicenter MEN1 cohort study showed the presence of gastrinomas and PanNETs $>2 \mathrm{~cm}$ were both independently associated with distant metastases, only metastasized PanNETs $>2 \mathrm{~cm}$ subsequently increased the risk of death (26). Given the good prognosis, conservative symptomatic treatment with PPI might be favored, especially as major duodenopancreatic 
surgery - necessary to achieve long-term cure - is associated with severe morbidity $(6,27$ 29). On the other hand, liver metastases can also cause significant morbidity. Furthermore, several other studies describe a subgroup of aggressive MEN1-associated gastrinomas (10$25 \%$ of patients) with worse survival $(30,31)$. Aggressive disease was associated with higher gastrin levels, liver metastases, concurrent PanNET size and fast tumor growth, but not with the presence of $\operatorname{LNM}(30,31)$. Those with aggressive disease can potentially benefit from a major duodenopancreatic resection that can cure gastrinoma disease. Patients undergoing limited resections, i.e., duodenotomy with local resection, often have persistent or recurrent hypergastrinemia either due to missed or new gastrinomas in the remnant duodenum (3, 32). The high probability of persistent disease is underscored by the very small gastrinomas $(0.05-0.20 \mathrm{~cm})$ in the present study (33).

With the current findings, MEN1 patients with hypergastrinemia, a NET in the head of the pancreas and radiological signs of peri-duodenopancreatic LNM, a duodenal origin of the LNM should be considered, which is consistent with previous literature $(19,20)$. Since periduodenopancreatic LNM in patients with MEN1-related hypergastrinemia are most likely of duodenal origin, a major duodenopancreatic resection is necessary to cure both duodenal and pancreatic disease. Nevertheless, in patients with non-aggressive duodenal gastrinoma LNM (i.e., with mild hypergastrinemia and slow growth rate), preventing postoperative morbidity by non-surgical treatment versus a major duodenopancreatic resection might outweigh the risk of distant gastrinoma metastases - estimated at $10 \%$ - and gastrinomarelated death $(24,26,27,31)$.

Only few non-functional PanNETs with metastases were included, so it was not possible to study the association of ARX/PDX1 expression and metastatic behavior, which was previously shown in MEN1-associated NF-PanNETs (9). The relative abundance of sole ARX expressing microadenomas as compared to PanNETs is consistent with the higher proportion of glucagon (alpha cell, ARX+/PDX1- $(34,35)$ ) expressing microadenomas seen in patients with MEN1 (20). Nevertheless, the unexpected large proportion of low-grade PanNETs and microadenomas without PDX1 expression (Table 2), previously associated with the development of liver metastases (9), could hamper the pre-operative applicability of these biomarkers. In this respect, WHO grade more accurately separated indolent from malignant PanNETs. Three G2 PanNETs clustered with presumed pancreatic metastases (patients no. M1, M6, and M7), and only four other dpNETs were G2. ALT was seen in a single PanNET with metastases, and in none of the duodenal NETs and gastrin positive 
peri-duodenopancreatic LNMs. Interestingly, in the same patient a G1 microadenoma showed the ALT phenotype (36). A microadenoma with ALT has not been reported in MEN1 before, although an intrapancreatic metastasis cannot be excluded. The retained ATRX/ DAXX expression can be explained by point mutations, translocations, or non-ATRX/DAXX mutations causing the ALT phenotype (37).

The major strength of the present study includes the extensive histopathological analysis of individual dpNETs and metastases in a well-defined MEN1 cohort. Despite the rarity of MEN1, 10 patients undergoing pancreatoduodenectomies or total pancreatectomies were included leading to a high number of dpNETs to enable robust and comprehensive (histopathological) analysis. Limitations include the fact that only highly selected patients undergo major duodenopancreatic surgery or metastasectomies. Surgical indications are ambiguous and particularly for MEN1-associated gastrinomas surgical indications are equivocal. This limits generalizability of these results to all other MEN1 patients. Moreover, most patients have remaining pancreatic, duodenal or pyloric tissue, often harboring additional dpNETs (Table 1). However, it is assumed that dominant and clinically relevant disease was removed during surgery. Finally, only two markers were used as surrogate markers for clonal relations, therefore, a coincidental similar expression cannot not be excluded. Indeed, often more than one primary dpNET clustered with the metastases. Therefore, assigning a single tumor of origin (or the sequence of events) without taking known risk factors, such as tumor size and grade, into consideration remains challenging. Nevertheless, even without knowing the exact tumor of origin, the results show clear transcription factor proximity of peri-duodenopancreatic LNM to duodenal gastrinomas and not a single PanNET and are backed by the expression of gastrin.

In conclusion, this study provides detailed insight in the metastatic spread of dpNETs in a group of patients with resected multifocal MEN1-associated dpNETs. In six patients with MEN1-related hypergastrinemia, all peri-duodenopancreatic LNM originated from duodenal gastrinomas based on ARX, PDX1 and gastrin expression. Duodenal gastrinomas were often small, and not always detected by imaging preoperatively. Therefore, this study underlines that a duodenal origin of peri-duodenopancreatic LNM should be considered in all patients with MEN1-associated hypergastrinemia, also when clinically relevant NETs are present in the pancreatic head. 


\section{REFERENCES}

1. Ito $T$, Igarashi $H$, Uehara $H$, Berna MJ, Jensen RT. Causes of death and prognostic factors in multiple endocrine neoplasia type 1: a prospective study: comparison of 106 MEN1/ Zollinger-Ellison syndrome patients with 1613 literature MEN1 patients with or without pancreatic endocrine tumors. Medicine (Baltimore). 2013;92(3):135-81.

2

Thakker RV, Newey PJ, Walls GV, Bilezikian J, Dralle $\mathrm{H}$, Ebeling PR, et al. Clinical practice guidelines for multiple endocrine neoplasia type 1 (MEN1). J Clin Endocrinol Metab. 2012;97(9):2990-3011

3. Falconi M, Eriksson B, Kaltsas G, Bartsch DK, Capdevila J, Caplin M, et al. ENETS Consensus Guidelines Update for the Management of Patients with Functional Pancreatic Neuroendocrine Tumors and Non-Functional Pancreatic Neuroendocrine Tumors. Neuroendocrinology. 2016;103(2):153-71.

4. Howe JR, Merchant NB, Conrad C, Keutgen XM, Hallet J, Drebin JA, et al. The North American Neuroendocrine Tumor Society Consensus Paper on the Surgical Management of Pancreatic Neuroendocrine Tumors. Pancreas. 2020;49(1):1-33. 5. Sadowski SM, Millo C, Cottle-Delisle C, Merkel R, Yang LA, Herscovitch P, et al. Results of (68)Gallium-DOTATATE PET/CT Scanning in Patients with Multiple Endocrine Neoplasia Type 1. J Am Coll Surg. 2015;221(2):509-17.

6. Nell S, Borel Rinkes IHM, Verkooijen HM, Bonsing BA van Eijck $\mathrm{CH}$, van Goor H, et al. Early and Late Complications After Surgery for MEN1-related Nonfunctioning Pancreatic Neuroendocrine Tumors. Ann Surg. 2018;267(2):352-6.

7. van Beek DJ, Nell S, Vorselaars W, Bonsing BA, van Eijck CHJ, van Goor H, et al. Complications After Major Surgery for Duodenopancreatic Neuroendocrine Tumors in Patients with MEN1: Results from a Nationwide Cohort. Ann Surg Oncol. 2021. 8. Chan CS, Laddha SV, Lewis PW, Koletsky MS, Robzyk K, Da Silva E, et al. ATRX, DAXX or MEN1 mutant pancreatic neuroendocrine tumors are a distinct alpha-cell signature subgroup. Nat Commun. 2018;9(1):4158.

9. Cejas P, Drier Y, Dreijerink KMA, Brosens LAA, Deshpande $V$, Epstein $C B$, et al. Enhancer signatures stratify and predic outcomes of non-functional pancreatic neuroendocrine tumors. Nat Med. 2019;25(8):1260-5.

10. Boons G, Vandamme T, Ibrahim J, Roeyen G, Driessen A, Peeters D, et al. PDX1 DNA Methylation Distinguishes Two Subtypes of Pancreatic Neuroendocrine Neoplasms with a Different Prognosis. Cancers (Basel). 2020;12(6).

11. Hackeng WM, Schelhaas W, Morsink FHM, Heidsma CM, van Eeden S, Valk GD, et al. Alternative Lengthening of Telomeres and Differential Expression of Endocrine Transcription Factors Distinguish Metastatic and Non-metastatic Insulinomas. Endocr Pathol. 2020.

12. Hackeng WM, Brosens LAA, Kim JY, O'Sullivan R, Sung YN, Liu TC, et al. Non-functional pancreatic neuroendocrine tumours: ATRX/DAXX and alternative lengthening of telomeres (ALT) are prognostically independent from ARX/PDX1 expression and tumour size. Gut. 2021.

13. van Beek DJ, van Leeuwaarde RS, Pieterman CR,

Vriens MR, Valk GD. 'Quality in, quality out', a stepwise approach to EBM for rare diseases promoted by MEN1. Endocr Connect. 2018.
14.

van Beek DJ, Verkooijen HM, Nell S, Bonsing BA, van Eijck CH, van Goor H, et al. Reliability and Agreement of Radiological and Pathological Tumor Size in patients with MEN1-Related Pancreatic Neuroendocrine Tumors: Results from a Population-Based Cohort. Neuroendocrinology. 2020.

15. Conemans EB, Brosens LAA, Raicu-Ionita GM, Pieterman CRC, de Herder WW, Dekkers OM, et al. Prognostic value of WHO grade in pancreatic neuro-endocrine tumors in Multiple Endocrine Neoplasia type 1: Results from the DutchMEN1 Study Group. Pancreatology. 2017;17(5):766-72.

16. Lloyd RV, Osamura RY, Klöppel G, Rosai J. WHO Classification of Tumours of Endocrine Organs: International Agency for Research on Cancer; 2017.

17. Landis JR, Koch GG. The measurement of observer agreement for categorical data. Biometrics. 1977;33(1):159-74.

18. Anlauf M, Enosawa T, Henopp T, Schmitt A, Gimm O, Brauckhoff $M$, et al. Primary lymph node gastrinoma or occult duodenal microgastrinoma with lymph node metastases in a MEN1 patient: the need for a systematic search for the primary tumor. Am J Surg Pathol. 2008;32(7):1101-5.

19. Pipeleers-Marichal M, Somers G, Willems G, Foulis A, Imrie C, Bishop AE, et al. Gastrinomas in the duodenums of patients with multiple endocrine neoplasia type 1 and the Zollinger-Ellison syndrome. N Engl J Med. 1990;322(11):723-7. 20. Anlauf M, Schlenger R, Perren A, Bauersfeld J, Koch $\mathrm{CA}$, Dralle $\mathrm{H}$, et al. Microadenomatosis of the endocrine pancreas in patients with and without the multiple endocrine neoplasia type 1 syndrome. Am J Surg Pathol. 2006;30(5):560-74.

21. Kimura H, Ohtsuka T, Fujimoto T, Date K, Matsunaga T, Cases Al, et al. Different Hormonal Expression Patterns Between Primary Pancreatic Neuroendocrine Tumors and Metastatic Sites. Pancreas. 2016;45(7):947-52.

22. Debelenko LV, Zhuang Z, Emmert-Buck MR, Chandrasekharappa SC, Manickam P, Guru SC, et al. Allelic deletions on chromosome 11q13 in multiple endocrine neoplasia type 1 -associated and sporadic gastrinomas and pancreatic endocrine tumors. Cancer Res. 1997;57(11):2238-43.

23. Niederle B, Selberherr A, Bartsch D, Brandi ML, Doherty GM, Falconi M, et al. Multiple Endocrine Neoplasia Type 1 (MEN1) and the Pancreas - Diagnosis and Treatment of Functioning and Non-Functioning Pancreatic and Duodenal Neuroendocrine Neoplasia within the MEN1 Syndrome - An International Consensus Statement. Neuroendocrinology. 2020.

24. Norton JA, Alexander HR, Fraker DL, Venzon DJ, Gibril F, Jensen RT. Comparison of surgical results in patients with advanced and limited disease with multiple endocrine neoplasia type 1 and Zollinger-Ellison syndrome. Ann Surg. 2001;234(4):495-505; discussion -6.

25. Jensen RT, Berna MJ, Bingham DB, Norton JA. Inherited pancreatic endocrine tumor syndromes: advances in molecular pathogenesis, diagnosis, management, and controversies. Cancer. 2008;113(7 Suppl):1807-43.

26. Vinault S, Mariet AS, Le Bras M, Mirallie E, Cardot-Bauters C, Pattou F, et al. Metastatic Potential and Survival of Duodenal and Pancreatic Tumors in Multiple Endocrine Neoplasia Type 1: A GTE and AFCE Cohort Study (Groupe d'etude des 


\section{Chapter 9}

Tumeurs Endocrines and Association Francophone de Chirurgie Endocrinienne). Ann Surg. 2020;272(6):1094-101.

27. Lopez CL, Falconi M, Waldmann J, Boninsegna L, Fendrich V, Goretzki PK, et al. Partial pancreaticoduodenectomy can provide cure for duodenal gastrinoma associated with multiple endocrine neoplasia type 1. Ann Surg. 2013;257(2):308-14. 28. Tonelli F, Fratini G, Nesi G, Tommasi MS, Batignani G, Falchetti $A$, et al. Pancreatectomy in multiple endocrine neoplasia type 1-related gastrinomas and pancreatic endocrine neoplasias. Ann Surg. 2006;244(1):61-70.

29. Dickson PV, Rich TA, Xing Y, Cote GJ, Wang H, Perrier ND, et al. Achieving eugastrinemia in MEN1 patients: both duodenal inspection and formal lymph node dissection are important. Surgery. 2011;150(6):1143-52.

30. Gibril F, Venzon DJ, Ojeaburu JV, Bashir S, Jensen RT. Prospective study of the natural history of gastrinoma in patients with MEN1: definition of an aggressive and a nonaggressive form. J Clin Endocrinol Metab. 2001;86(11):5282-93.

31. van Beek DJ, Nell S, Pieterman CRC, de Herder WW, van de Ven AC, Dekkers OM, et al. Prognostic factors and survival in MEN1 patients with gastrinomas: Results from the DutchMEN study group (DMSG). J Surg Oncol. 2019;120(6):966-75. 32. Jensen RT, Cadiot G, Brandi ML, de Herder WW,
Kaltsas G, Komminoth P, et al. ENETS Consensus Guidelines for the management of patients with digestive neuroendocrine neoplasms: functional pancreatic endocrine tumor syndromes. Neuroendocrinology. 2012;95(2):98-119.

33. Thompson NW, Vinik Al, Eckhauser FE. Microgastrinomas of the duodenum. A cause of failed operations for the Zollinger-Ellison syndrome. Ann Surg. 1989;209(4):396-404.

34. Baron M, Veres A, Wolock SL, Faust AL, Gaujoux R Vetere A, et al. A Single-Cell Transcriptomic Map of the Human and Mouse Pancreas Reveals Inter- and Intra-cell Population Structure. Cell Syst. 2016;3(4):346-60 e4.

35. Muraro MJ, Dharmadhikari G, Grun D, Groen N Dielen T, Jansen E, et al. A Single-Cell Transcriptome Atlas of the Human Pancreas. Cell Syst. 2016;3(4):385-94 e3.

36. de Wilde RF, Heaphy CM, Maitra A, Meeker AK, Edi $\mathrm{BH}$, Wolfgang $\mathrm{CL}$, et al. Loss of ATRX or DAXX expression and concomitant acquisition of the alternative lengthening of telomeres phenotype are late events in a small subset of MEN-1 syndrome pancreatic neuroendocrine tumors. Mod Pathol. 2012;25(7):10339.

37. Brosnan-Cashman JA, Graham MK, Heaphy CM. Genetic alterations associated with ALTered telomeres. Oncotarget. 2018;9(73):33739-40. 


\section{SUPPLEMENTARY FIGURES AND TABLES}

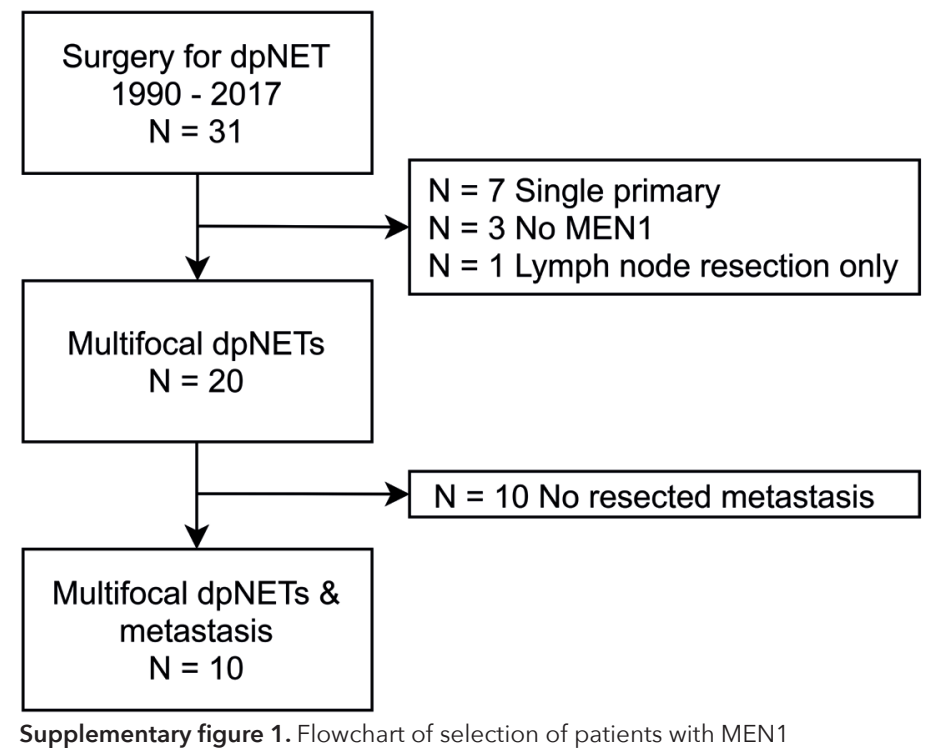




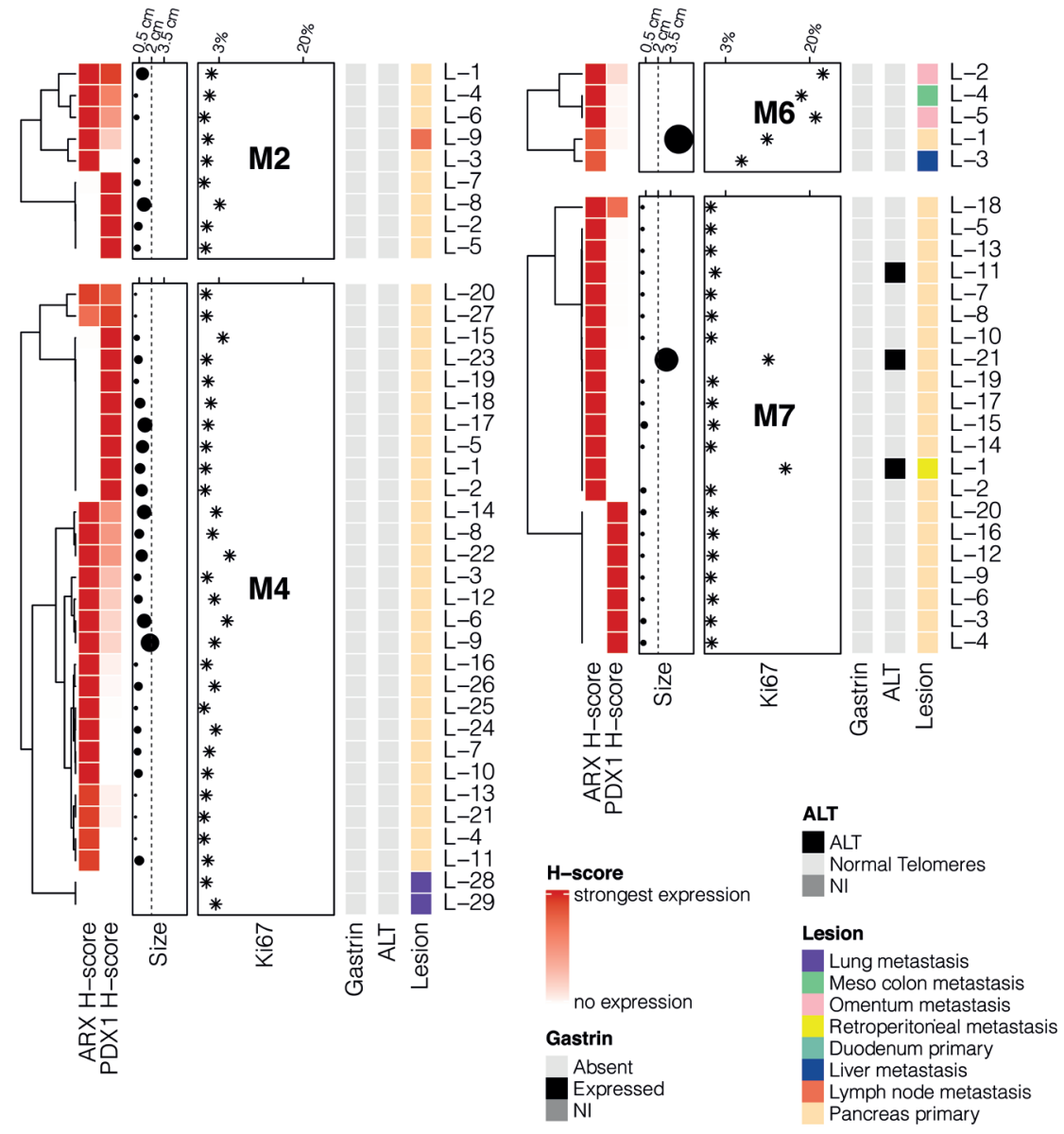

Supplementary figure 2. Metastatic dpNET pattern in additional patients with MEN1. Metastatic patterns of patients M2, M4, M6, and M7. Unsupervised hierarchical clustering is performed on the ARX and PDX1 H-score heatmap. An H-score is the immunohistochemical nuclear staining intensity multiplied with the percentage of staining cells. Lesions are annotated with size (except metastases), Ki-67 labeling index, gastrin expression, the presence of Alternative Lengthening of Telomeres (ALT), and anatomic location. 


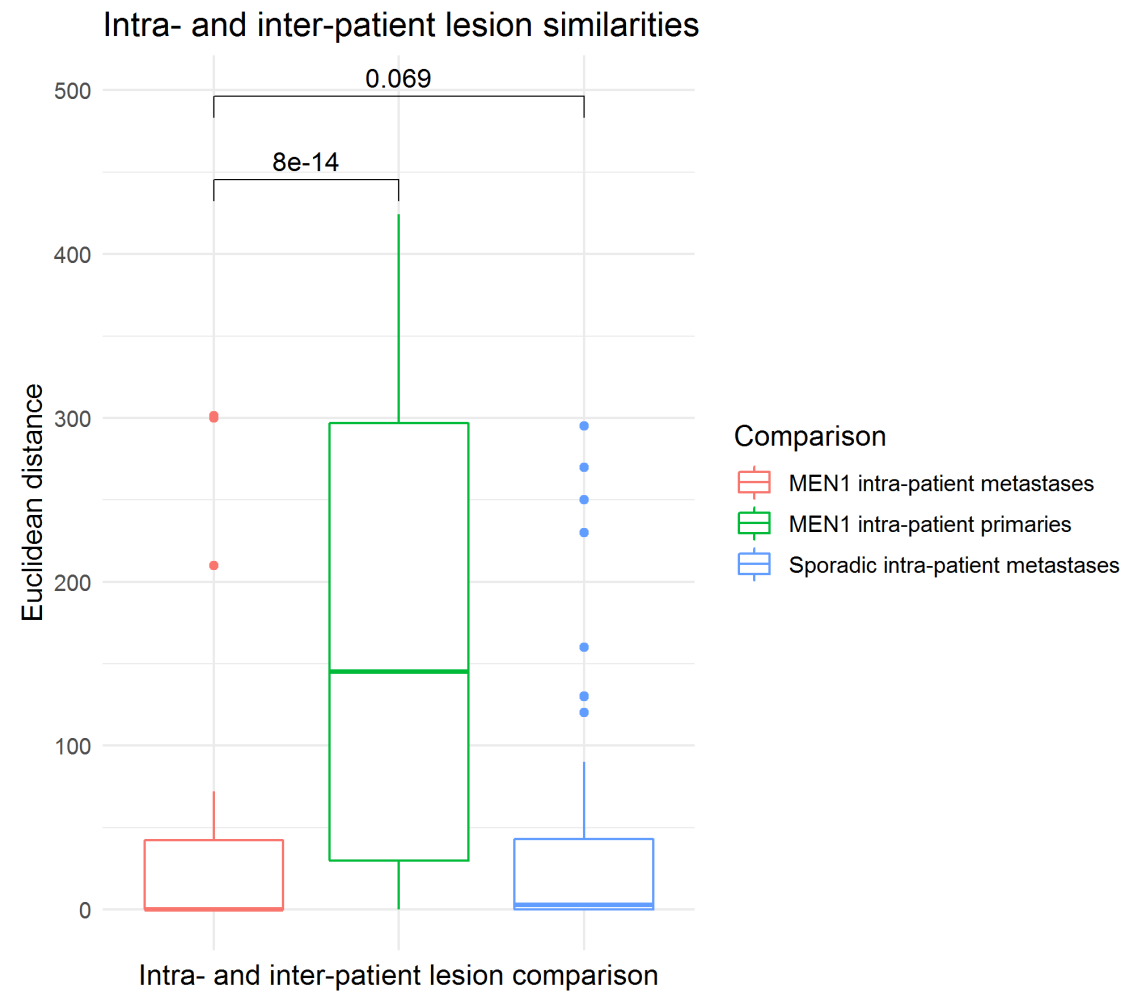

Supplementary figure 3. Intra-patient Euclidean distance comparison. Intra-patient Euclidean distance comparison for sporadic metastases, MEN1-associated primary tumors, and MEN1-associated metastases 

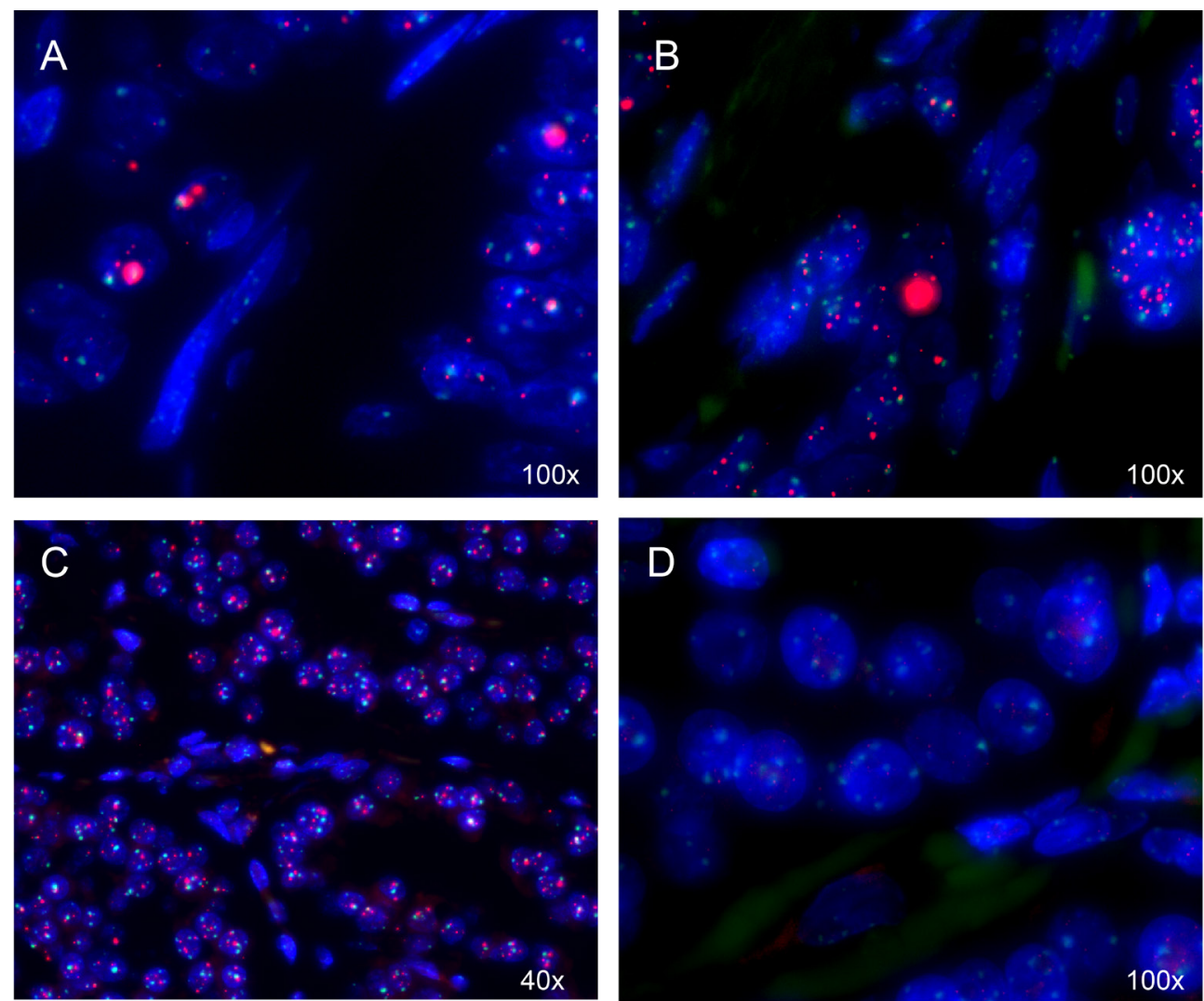

\section{DAPI}

Telomere

\section{Centromere}

Supplementary figure 4. Telomere fluorescence in situ hybridization in patients with MEN1. A,B,D 100x magnification. C 40x magnification. A, alternative lengthening of telomeres (ALT) in NET (patient no. M7, L-21). Ultrabright telomeric signals are $>10 x$ the signal intensity of the cumulative signal intensities of nearby stromal cells (spindle shaped). B, ALT in retroperitoneal NET metastasis (patient no. M7, L-1). C, ALT in microadenoma (patient no. M7, L-11). L, normal telomeres in pancreatic NET (patient no. M2, L-8). 
Supplementary Table 1. Baseline characteristics

\begin{tabular}{|c|c|}
\hline Characteristics & Overall $n=10$ \\
\hline Age at surgery in years, median [range] & $50[31-63]$ \\
\hline \multicolumn{2}{|l|}{ Sex } \\
\hline Male & $5(50 \%)$ \\
\hline Female & $5(50 \%)$ \\
\hline \multicolumn{2}{|l|}{ Surgical indication } \\
\hline NF-PanNET & $2(20 \%)$ \\
\hline Insulinoma & $2(20 \%)$ \\
\hline Gastrinoma & $3(30 \%)$ \\
\hline NF-PanNET and gastrinoma & $3(30 \%)$ \\
\hline Size largest PanNET on conventional imaging in $\mathrm{mm}$, median [range] & $20[3-48]$ \\
\hline PanNET $\geq 2 \mathrm{~cm}$ on preoperative imaging & $5(50 \%)$ \\
\hline Pancreatic head & $1(10 \%)$ \\
\hline Pancreatic body/tail & $3(30 \%)$ \\
\hline Pancreatic head and body/tail & $1(10 \%)$ \\
\hline \multicolumn{2}{|l|}{ Number of PanNETs on preoperative imaging } \\
\hline 1 & $1(10 \%)$ \\
\hline 2 & $1(10 \%)$ \\
\hline$\geq 3$ & $8(80 \%)$ \\
\hline \multicolumn{2}{|l|}{ Number of PanNETs head on preoperative imaging } \\
\hline 0 & $1(10 \%)$ \\
\hline 1 & $3(30 \%)$ \\
\hline 2 & $4(40 \%)$ \\
\hline$\geq 3$ & $2(20 \%)$ \\
\hline \multicolumn{2}{|l|}{ Number of PanNETs body/tail on preoperative imaging } \\
\hline 1 & $4(40 \%)$ \\
\hline 2 & $2(20 \%)$ \\
\hline$\geq 3$ & $4(40 \%)$ \\
\hline Suspected lymph node metastases on imaging & $7(70 \%)$ \\
\hline Fasting serum gastrin levels (Factor ULN) & $2.7[0.5-42.9]$ \\
\hline \multicolumn{2}{|l|}{ Procedures } \\
\hline Distal pancreatectomy & $3(30 \%)$ \\
\hline Whipple/PPPD & $2(20 \%)$ \\
\hline Whipple/PPPD + distal pancreatectomy & $3(30 \%)$ \\
\hline Total or completion pancreatectomy & $2(20 \%)$ \\
\hline
\end{tabular}

Abbreviations: ULN upper limit of normal, N number of, NF-PanNET non-functioning pancreatic neuroendocrine tumor, PanNET pancreatic neuroendocrine tumor, PPPD pylorus-preserving pancreatoduodenectomy 

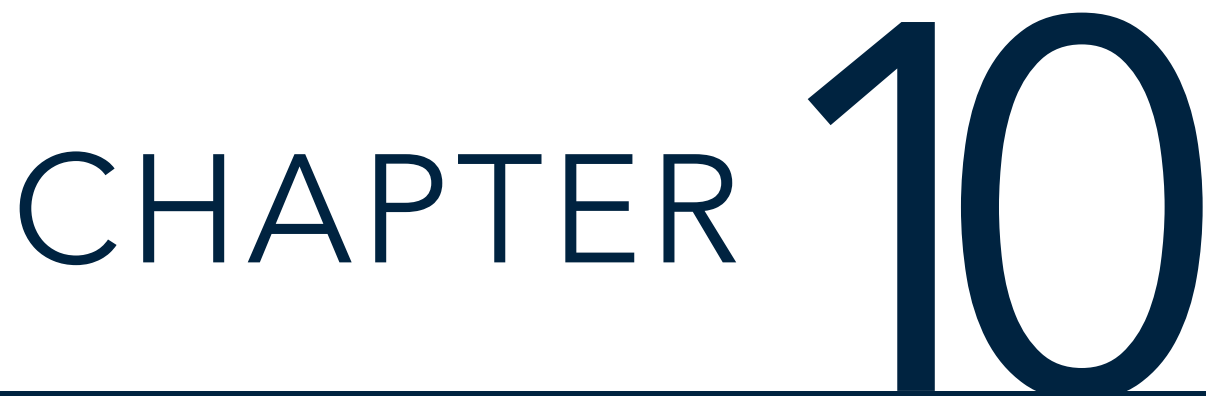

Genome methylation accurately predicts neuroendocrine tumor origin - an online tool 


\section{ABSTRACT}

The primary origin of neuroendocrine tumor metastases can be difficult to determine by histopathology alone, but is critical for therapeutic decision making. DNA methylation-based profiling is now routinely used in the diagnostic workup of brain tumors. This has been enabled by the availability of cost-efficient array-based platforms. We have extended these efforts to augment histopathological diagnosis in neuroendocrine tumors.

We compiled data of 69 small-intestinal, pulmonary, and pancreatic neuroendocrine tumors. These data were used to build a ridge regression calibrated random forest classification algorithm (NEN-ID) that predicts the origin of tumor samples with high accuracy (> 95\%). The model was validated during $3 \times 3$ nested cross validation and tested in a local and external cohort ( $n=198$ cases). In addition, we show that our diagnostic approach is robust across a range of possible confounding experimental parameters such as tumor purity and array quality. A software infrastructure and online user interface was built to make the model available to the scientific community.

This DNA methylation-based prediction model can be used in the workup for patients with neuroendocrine tumors of unknown primary. To facilitate validation and clinical implementation, we provide a user-friendly, publicly available web-based version of NEN-ID. 


\section{STATEMENT OF TRANSLATIONAL RELEVANCE:}

One out of nine patients with neuroendocrine tumors (NET) presents with metastases of unknown primary. The value of immunohistochemical panels to determine NET origin in these cases is limited. In this article, we investigate the use of DNA methylation as a biomarker of origin, an approach which has been successfully applied in other cancer types. Using widely available array-based methylation profiling, we provide evidence that our one-stop test is superior to traditional immunohistochemical techniques in determining small-intestinal, pulmonary, or pancreatic NET origin. We systematically validated our results through cross-validation, both in local and external test cohorts. The classifier presented here (NEN-ID) has already been successfully applied in the diagnostic workup of patients with NETs of unknown primary and is available as an open-source web application. This platform offers full analysis, classification and clinical report generation for NET methylation data, facilitating implementation and validation in clinical practice. 


\section{INTRODUCTION}

Neuroendocrine tumors (NET) have an incidence of about 6 per 100,000 per year and regularly present with metastases at diagnosis (1). In about one in nine NET patients, the primary tumor is unknown (2). As lymphoid, hepatic and pulmonary metastases can originate from any organ site in the body, the diagnostic workup can be challenging. Furthermore, discerning pulmonary metastases from primary pulmonary NETs is difficult, as these can appear morphologically and immunohistochemically similar. Also, for hereditary NET syndrome patients, who are at risk of developing synchronous primary NETs, the primary origin of metastases is often unclear. It is crucial to know the primary origin, since it determines prognosis, relevance of prognostic markers, treatment options, and eligibility for clinical trials $(1,3,4)$. Guidelines recommend that immunohistochemical panels should be performed on the metastatic tumor tissue, which can give clues to the primary site of origin, and guide or confirm imaging results and additional diagnostic techniques(5). However, diagnostic sensitivity and specificity varies greatly for the immunohistochemical panels, and the primary tumor is often not visible on imaging. A reliable one stop test to accurately determine primary origin is needed.

DNA methylation is important in regulating gene expression and the distribution of DNA methylation across the genome is highly tissue and tumor specific (6). Previously, whole genome methylation-profiling has successfully been applied to classify brain tumors and identify origin of carcinomas of unknown primary (7-9). This method has not yet been explored for NETs. Compared to the traditional immunohistochemical panels, genome methylation-based prediction models take advantage of combining many thousands of measurements at once and lack inter-observer bias, which improves the accuracy. To test the feasibility of this approach for NETs, we developed a methylation-profiling based prediction model and determined the accuracy in predicting the most common primary sites of origin for NET metastases, which are the small intestine, lung and pancreas. The Illumina methylation platforms ( $450 \mathrm{k}$ and EPIC) were chosen to assess whole genome methylation, as these arrays are widely available and have reproducible results (8). To make validation and clinical implementation attainable, we subsequently developed the Neuroendocrine Neoplasm Identifier (NEN-ID) web application to make our analysis pipeline available to the research and clinical NET community via an online interface. 


\section{MATERIALS AND METHODS}

\section{Methylation Cohorts}

The University Medical Center Utrecht (UMCU) Biobank Research Ethics Committee approved the use of archival material for this study. The methylation training cohort was compiled of publicly available methylation Illumina 450k data of small-intestinal, pulmonary, and pancreatic NETs (Fig. S1, Gene Expression Omnibus [GEO] accession numbers GSE73832, GSE118133, and GSE117852, respectively (10-12)). For small-intestinal NETs, supplementary data and data deposited in the GEO repository were matched by age, sex, grade, tissue-type and origin. Only primary formalin fixed paraffin embedded NET samples of certain ileal, terminal ileal or ileocaecal origin were selected $(n=19)$ to ensure a homogenous training population (10). NET origins with $<5$ cases were excluded. All pancreatic NETs $(n=32)$ and pulmonary $(n=18)$ were included in the further analysis $(11,12)$. As a "local test cohort", the UMCU pathology archive was screened for surgical specimens of primary NETs and NET metastases of small-intestinal, pulmonary, pancreatic and unknown primary origin. Cases of unknown primary were defined as unknown primary tumor location after routine clinical work-up including imaging. Additional small-intestinal, pulmonary, and pancreatic NETs were retrieved from GEO repository and the European Genome-phenome Archive (EGA) and combined to a "external test cohort" (accession numbers GSE73832 and GSE53051, GSE134089, EGAD00010001720 (10, 13-15), Fig. S1). For the simulated nontumor contamination, publicly available data of purified lymphocytes, normal pancreas and lung were downloaded (GEO accession GSE103541 (16) and TCGA). Methylation data of rare NETs were retrieved from previous studies (GSE73832 and GSE134089) and data of non-NET tumor and normal samples were retrieved from the TCGA database.

\section{Sample methylation array processing}

For the local test cohort, consisting of cases from our own institution ( $n=26)$, DNA extraction, bisulfite conversion and array processing were performed in-house (core facility). $4 \mu \mathrm{m}$ unstained sections were freshly cut for DNA extraction. Tumor tissue was macro-dissected if normal tissue was present in the slide. DNA was extracted after Proteinase $\mathrm{K}$ digestion using the Maxwell RSC 48 automated nucleic acid extraction platform (Promega, Madison, USA). Further steps were performed according to the protocols provided by the DNA methylation BeadChip manufacturer (Illumina, San Diego, USA). Briefly, bisulfite conversion was performed using the EZ DNA Methylation Kit (Zymo research, Irvine, USA). Formalin fixation induced DNA damage was restored using the FFPE Restore kit (Illumina). Whole-genome 
amplification on the bisulfite converted and restored DNA was performed, followed by fragmentation, precipitation and hybridization to CpG site specific beads (Illumina). After clearing unhybridized and nonspecifically hybridized DNA, BeadChips were extended, stained, coated and scanned using the iScan software (Illumina). Raw data were exported as IDAT files for further processing.

\section{Immunohistochemistry}

$4 \mu \mathrm{m}$ unstained sections of the local cases were cleared and deparaffinized. Slides were processed using the BenchMark ULTRA autostainer (Ventana Medical Systems, Oro Valley, USA) with 24 minutes Cell Conditioning 1 antigen retrieval at $100^{\circ} \mathrm{C}$. Slides were incubated with CDX2 (Clone EPR2764Y, cat. no. ILM2353-C1, 1:200; Immunologic, Duiven, The Netherlands) and TTF1 (Clone SPT24, RRID: AB_442138, 1:60; Leica Biosystems, Amsterdam, the Netherlands) at $36^{\circ} \mathrm{C}$ for 32 minutes, and visualized with Optiview DAB (Ventana Medical Systems). Islet-1 immunohistochemistry was performed manually. Briefly, peroxidase activity was blocked with $0.6 \% \mathrm{H} 2 \mathrm{O} 2$ in methanol. Slides were cooked in $10 \mathrm{mM}$ citrate ( $\mathrm{pH}$ 6) buffer for 20 minutes. After applying Pierce Protein-Free Blocking buffer (Thermo Fisher, Waltham, USA), slides were incubated with Islet-1 antibody (Clone EP283, cat. no. 431R-15, 1:400; Cell-Marque, Rockling, USA) in Normal Antibody Diluent (Immunologic) at $20^{\circ} \mathrm{C}$ for 60 minutes. The signal was amplified using the BrightVision+ Poly- HRP-Anti Mouse/Rabbit IgG Biotin-free kit (Immunologic), and detected with BrightDAB (Immunologic) for 8 minutes. Slides were counterstained with hematoxylin. Scoring was performed in consensus by a NET researcher (WMH) and experienced NET pathologist (LAAB).

\section{Data analysis}

All analyses were performed using R version 3.6.3 (17). The following sections provide a non-exhaustive overview of the most important analytical steps. The full code used to produce figures and results presented in the manuscript is available for inspection on Github (whackeng/NEN-ID). Methylation data has been deposited at the European Genomephenome Archive (EGA), which is hosted by the EBI and the CRG, under accession number EGAS00001004878.

\section{Preprocessing}

The ChAMP package was used to load and process raw data (18). Failed probes with a detection $\mathrm{p}$-value of more than 0.01 were removed. Probes were filtered if they fulfilled any of the following criteria: beadcount $<3$ in at least $5 \%$ of samples, non-CpG targets or 
location on the X/Y chromosomes. Furthermore, all SNP-containing and multi-hit probes were removed. Ileal NETs were not filtered for beadcount as raw data was not available for these cases. Samples were normalized using the BMIQ method, as this approach was used for the pre-processed small-intestinal NET samples (10). In addition, sample quality was assessed by visual inspection of the sample density plots and failed CpG fraction.

\section{Probe selection and unsupervised analysis}

Training datasets were randomly downsampled 3 times stratified by tumor origin. For each downsampled dataset, probes were ordered by the mean absolute standard deviation. The 5000 most variable probes were selected by the minimal rank of the mean absolute standard deviation over all downsampled datasets. Unsupervised hierarchical clustering was performed on the most informative probes using the Euclidean distance. The t-Distributed Stochastic Neighbor Embedding visualization was made with the Rtsne package on the most variable probes of the entire training cohort (19).

\section{Random forest classifier training and ridge logistic regression calibration}

Data was filtered for CPG probes present in both the 450k array and the EPIC array. The 5000 most variable probes selected during triplicate downsampling were used as input variables. The random forest model was built using the randomForest package with 500 binary classification trees and default parameters. A downsampling strategy was used to balance classes present in the training cohort. For each decision tree, Breiman's random forest algorithm creates a randomly bootstrapped dataset with replacement (20). The class of each sample in the training set is predicted by the aggregated votes (bootstrap aggregation/bagging) of all random forest trees for which the specific sample was omitted from the bootstrapped training set (OOB votes), and for which the sample can be considered "unknown". The internal OOB votes from the random forest model were used to optimize the Lambda parameter though a three-fold cross validation using glmnet. The loss measured during cross-validation was deviance (default). A multinomial ridge penalized regression model was built using the optimized Lambda.1se parameter.

\section{$3 \times 3$ nested cross validation}

The entire training cohort was randomly split in three folds stratified for NET origin. During the outer loop 3-fold cross validation, each fold is held out once to use as test set, while the others are used as training set to fit a random forest model. Each fold is used for testing once. During the inner loop cross validation, the Lambda parameter is optimized on the OOB 
votes of the training fold. A ridge logistic regression model is fit on the all OOB votes using the optimized Lambda.1se parameter. The test fold held out of the outer cross validation loop is then tested using the raw random forest model, after which raw scores are calibrated using the logistic ridge regression model. The whole process of randomly splitting the entire training cohort into three folds and $3 \times 3$ nested cross validation is repeated three times. Output model metrics included overall majority vote- and probability-based model accuracy, multiclass area under the curve (AUC) (21) and log loss, and are presented as descriptive summary statistics. Furthermore, raw and calibrated scores were collected and used to calculate density plots. To plot the output of this 3 class problem, calibrated output of new cases was visualized using a ternary plot with ggtern (22). Tolerance ellipses were drawn on the calibrated $\mathrm{OOB}$ scores using the Mahalanobis distance and isometric log ratio transformation by an implementation of DrawMahal (23) in ggtern.

\section{Failed probe imputation}

The effect of randomly imputing a percentage of probes on accuracy was determined from $5 \%$ to $95 \%$ random imputation with steps of $5 \%$ on the complete set of beta values (around $400 \mathrm{k}$ ) in the local test cohort. Each time, for a specified percentage of probes the beta values were replaced with a random beta value selected from other probes from the same case, thus following a normal beta distribution. Imputed cases were tested with the raw and calibrated random forest model. This process was repeated 30 times. Mean and standard deviations of accuracy, multiclass AUC and log loss were plotted over incrementally increasing percentages of randomly imputed probes.

\section{Simulated normal cell contamination.}

Normalized data of sorted lymphocytes, normal pancreas and normal lung tissue was used to test the effect of non-tumor contamination on the model accuracy. First, mean beta values were calculated for B-cells, CD4-cells, CD8-cells, granulocytes and monocytes, normal pancreas, and normal lung. Next, a weighted average of the mean beta values of CD4 cells $\left({ }^{*} 0.55\right), \mathrm{B}$-cells $\left({ }^{\star} 0.35\right)$ and CD8 $\left({ }^{\star} 0.1\right)$ was calculated to simulate the average lymph node beta values. Percentages were based on the literature (24-27). To simulate contamination, a weighted average of mean normal cell population beta values was calculated with the beta values of each specific tumor. The percentage of the contamination determined the weight, which ranged from 5 to $95 \%$ with steps of $5 \%$. The local test cohort was used to test the effect of normal cell contamination. Similar to the random probe imputation, model metrics were plotted over incrementally increasing contamination percentages. 


\section{RESULTS}

\section{NETs of different origin show distinct methylomes}

We assembled a cohort of 69 NETs including 19 small-intestinal, 18 pulmonary, and 32 pancreatic NETs, which had been assayed on the microarray-based Illumina HumanMethylation450 platform (10-12)(Table 1, Fig. S1), further referred to as "entire training cohort". Characteristics of the cohort can be found in table S1. In addition, we generated 850k EPIC methylation array data for 26 NET samples from 23 patients from our own pathology archive (4 small-intestinal, 10 pulmonary, 9 pancreatic NETs, and 3 NETs of unknown primary) as a "local test cohort". For PanNET patients 5 and 6, primary tumors and matched metachronous liver metastasis ( +3 years, patient 5 ) and two metachronous liver metastases (+3 years, +6 years, patient 6 ) were included (Supplementary data 1). Dimensionality reduction through t-Distributed Stochastic Neighbor Embedding (t-SNE) on the most variable probes of the entire training cohort showed clustering of samples in the entire training cohort and our local test cohort, mostly corresponding with the three primary sites of origins (Fig. 1A). Few public data cases and one local sample cluster separately from the main origin clusters (Fig. 1A, unsupervised hierarchical clustering of the entire training cohort, Fig S2). These cases represent the "Lung Carcinoid Type 2" (LC2) subtype of pulmonary NETs which carry MEN1 mutations as presented by Laddha et al. (12). As the local test cohort comes from a single institution, and contains all three different sites of origin, the clustering with the anatomic locations in the entire training cohort suggests that the main source of variability in the methylation data is indeed origin of the tumor, rather than laboratory variance.

\section{Random forest models with calibration robustly predict NET origin}

Based on the observations in our unsupervised analysis, we trained a random forest model to distinguish the different origins. This machine learning technique builds an ensemble of decision trees and aggregates the class votes across the individual predictions into a final score. Data of the 5000 most variable probes was used as input to train the model (Supplementary data 2). Only probes both present in the Illumina 450k and EPIC array were used to make the model compatible for both platforms. Based on the out-of-bag (OOB) error, which is an internal random forest validation measure evaluated on held-out samples, the model showed a high accuracy of $97 \%$. To improve classification accuracy and make better comparison of class scores possible, a ridge penalized logistic regression calibration model was fitted to the random forest scores (28). Logistic regression is used to improve 
A

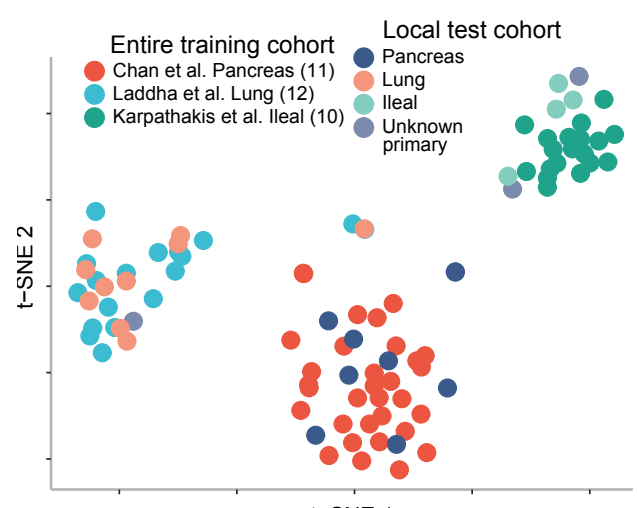

t-SNE 1

C

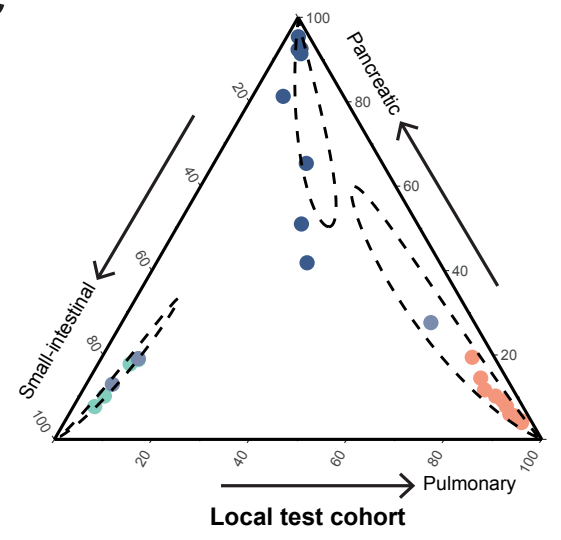

B

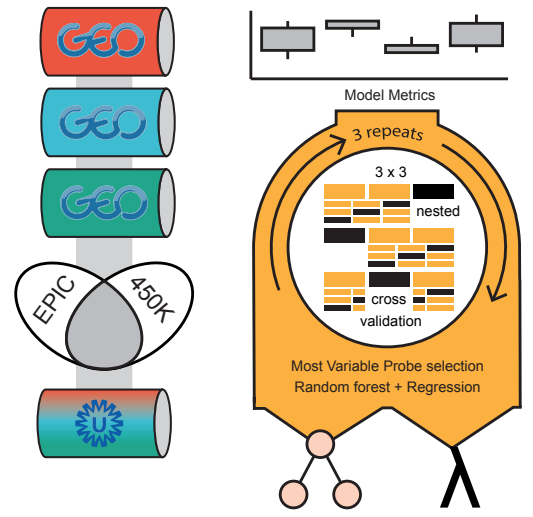

D

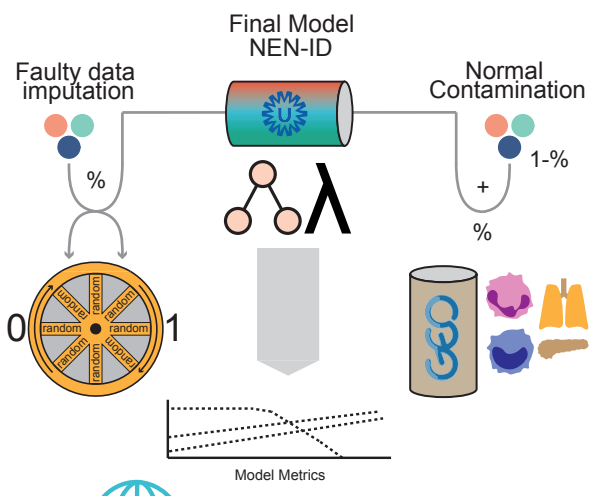

www.nen-id.com

Figure 1. Overview of performed analyses and local test cohort results. A, Unsupervised 2D t-SNE visualization of most variable probes in the entire training cohort, merged with the local test cohort. B, Workflow of the model validation, with a $3 \times 3$ nested cross-validation scheme with 3 repeats for random forest building and nested lambda optimization. Random forest and calibrated ridge regression output was tested on the internal test sets (black fold) and a test set of external cases (local test cohort). A number of model metrics such as classification accuracy, area under the curve and log-loss were investigated. C, NEN-ID classifier scores in ternary plot for the cases in the local test cohort, resulting in perfect classification accuracy. Tolerance ellipses are drawn for the calibrated out-of-bag scores for the model. D, the effect of erroneous data was tested in the local test cohort by random probe imputation for a percentage of probes. Similarly, the effect of normal cells (e.g., lymphocytes, normal lung) contaminating the tumor samples was simulated by mixing beta values of sorted or mixed normal cell populations with tumor samples. 
Table 1. Samples used for training or testing

\begin{tabular}{|c|c|c|c|c|c|c|c|c|c|c|c|}
\hline & \multicolumn{4}{|c|}{ Entire training cohort } & \multicolumn{7}{|c|}{ Local test cohort and external test cohort } \\
\hline & 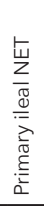 & 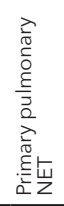 & 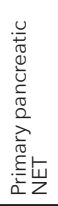 & 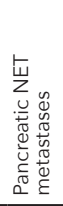 & 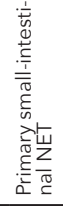 & 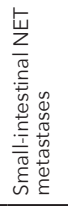 & 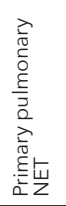 & 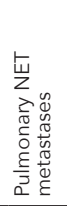 & 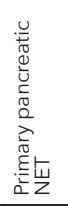 & 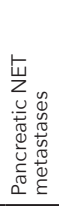 & 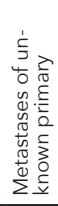 \\
\hline Karpathakis et al.(10) & 19 & & & & 16 & 21 & & & & & \\
\hline Laddha et al.(12) & & 18 & & & & & & & & & \\
\hline Chan et al.(11) & & & 23 & 9 & & & & & & & \\
\hline Local test cohort & & & & & 1 & 3 & 9 & 1 & 6 & 3 & 3 \\
\hline Timp et al.(13) & & & & & & & & & 5 & & \\
\hline Tirosh et al.(14) & & & & & 22 & & & & 50 & & 2 \\
\hline Alcala et al.(15) & & & & & & & 56 & & & & \\
\hline Subtotal & 19 & 18 & 32 & & 63 & & 66 & & 64 & & 5 \\
\hline Total & 69 & & & & 198 & & & & & & \\
\hline
\end{tabular}

NET, neuroendocrine tumor

class estimates by taking all random forest class scores into account to predict probability. The ridge penalty (Lambda) is selected during cross validation, to shrink the regression coefficients and prevent overfitting of the calibration model.

To test the generalizability of this model, a $3 \times 3$ nested cross validation (CV) was performed with 3 repeats (Fig. 1B) as previously described $(8,28)$. Briefly, 9 different randomly selected (sub)training and test cohorts were selected from the entire training cohort and used to build and test calibrated random forest models. In addition, our local test cohort $(n=23$ cases, with known origin) was included as separate test set for every training partition during cross validation. Each different training fold therefore consisted of approximately 46 cases (on average by random selection), while each combined test fold/local test cohort consisted of around 45 cases. The mean OOB accuracy during cross validation was $95 \%$. Median cross validation lambda penalty for the ridge logistic regression was 0.087 (range 0.064 - 0.113). Within the test folds and local test cohort during cross validation, the mean predictive accuracy of the corresponding calibrated random forest models was $96.1 \%$

Table 2. Model metrics of $3 \times 3$ nested internal and external cross-validation

\begin{tabular}{lllll}
\hline Samples & Model & Mean Accuracy (range) & Mean AUC (range) & Mean Log Loss (range) \\
\hline Internal Training & RF (OOB) & $0.9541(0.9348-0.9787)$ & $0.9964(0.9937-0.9993)$ & $0.2950(0.2405-0.3522)$ \\
Internal test fold & Raw RF & $0.9661(0.9130-1.0000)$ & $0.9989(0.9949-1.0000)$ & $0.2830(0.2315-0.3414)$ \\
Internal test fold & RF + LR & $0.9613(0.9130-1.0000)$ & $0.9989(0.9924-1.0000)$ & $0.1977(0.1601-0.2586)$ \\
Local test cohort & Raw RF & $0.9517(0.9130-0.9565)$ & $0.9986(0.9963-1.0000)$ & $0.4240(0.4083-0.4459)$ \\
Local test cohort & RF + LR & $0.9952(0.9565-1.0000)$ & $0.9998(0.9981-1.0000)$ & $0.2867(0.2508-0.3313)$ \\
\hline
\end{tabular}

RF, random forest model; OOB, 1-(out-of-bag error \%); LR, Ridge Logistic Regression model. AUC, multiclass area under the curve as defined by Hand and Till (21) 
and $99.5 \%$, respectively. Mean multiclass area under the curve ranged between 0.9924 and 1 (Table 2). Ridge logistic regression calibration improved accuracy in the local test cohort. Also, it caused a decrease in log loss values, meaning the correct class scores rise and incorrect class scores drop. We concluded by the near perfect performance of these predictive models in $3 \times 3$ cross validation that a random forest model built on this data is likely to perform well on unseen data.

To determine range of class scores to expect when classifying unknown data in the future, we kept track of the scores assigned to training cohort data test folds during cross validation (Fig. S3A, Supplementary Data 3). The true class score distribution was left skewed, and around 1/4 NET received true class scores within the left tail of the distribution (scores $\leq 80 \%$ ), indicating a broad range of expected true class scores. As expected, similar results were obtained from the calibrated OOB scores taken from the random forest model trained on the entire training cohort without cross validation (Fig. S3B).

\section{NEN-ID performance in the local test cohort}

We now tested the accuracy of the model trained on the entire training cohort (NEN-ID classifier) in our local test cohort. The random forest ridge regression calibrated output was $100 \%$ accurate in predicting origin of the 23 cases with a known origin (Fig. 1C, Table 3, Fig. S3C, Supplementary data 3 \& 4). Of note, one pulmonary NET from our local test cohort (Lung_NEN_sporadic5), that initially clustered with the LC2 carcinoids in the middle of the t-SNE (Fig. 1A), and showed chromosome 11 loss (locus MEN1), was correctly classified as pulmonary NET. Another case (Lung_NEN_sporadic3) with ectopic symptomatic ACTH production was also correctly classified (Supplementary data 1 \& 4). In comparison, an immunohistochemical panel to determine origin (TTF1, CDX2 and Islet-1) could only correctly classify $65-74 \%$ cases (15 or 17 out of 23 ), depending on the guidelines used for interpretation $(29,30)$ (Table 3$)$. The NEN-ID classifier was especially useful for triple negative cases or cases with more than one positive immunohistochemical marker. CDX2 positive pancreatic NETs showed the lowest scores, but were correctly classified nonetheless. As morphology can also be used to determine origin (31, 32), we determined NET growth patterns for the local test cohort. Although all small-intestinal NETs showed nested growth patterns, this pattern was also seen in other NETs. Spindle like cells were specific for lung origin, but were only seen few cases (Table 3, Supplementary data 1).

The three NETs of unknown primary from the local test cohort are highlighted in Fig. 2. 


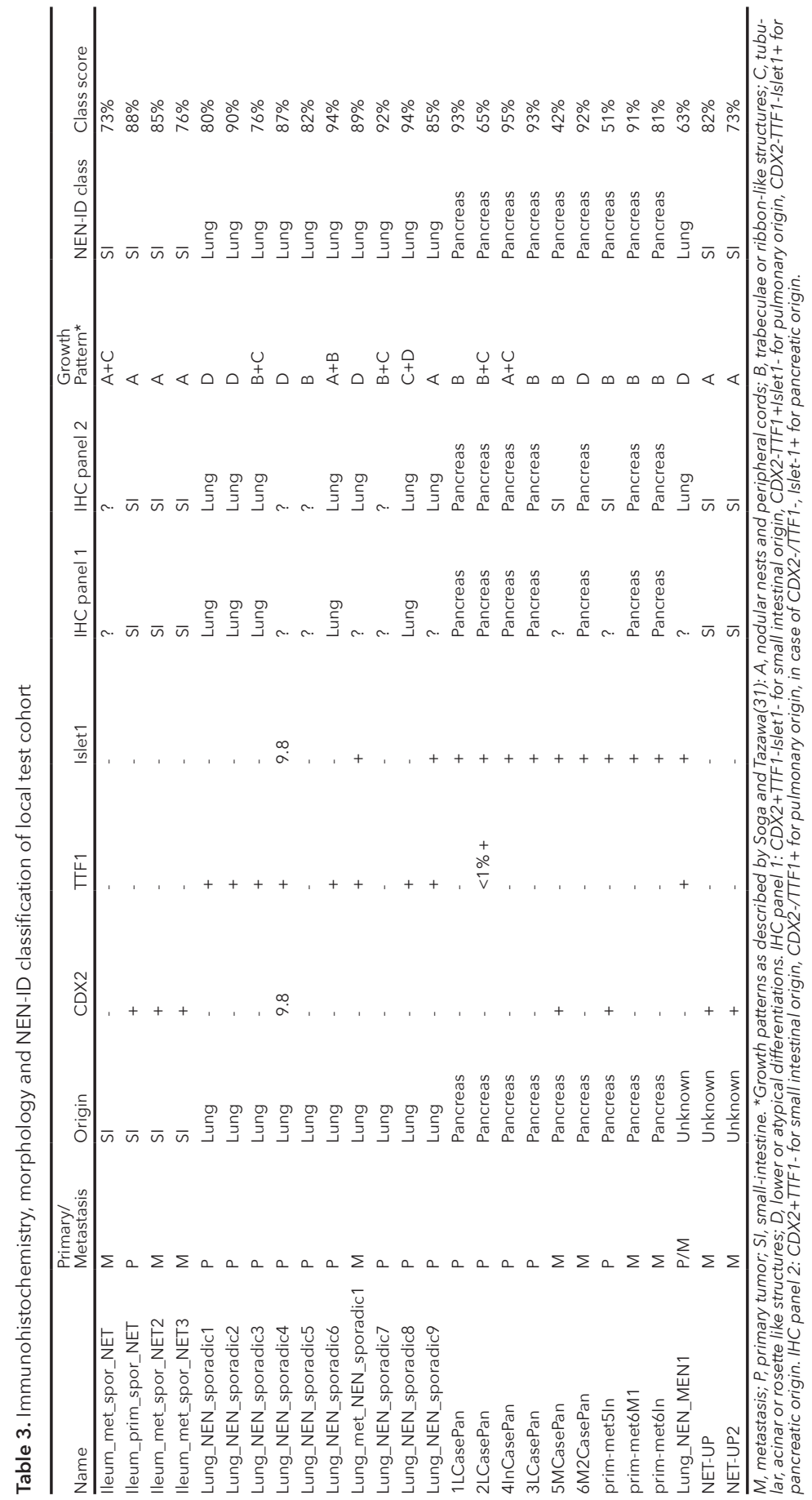


A

Path. diagnosis

Synaptophysin

Chromogranin A

CDX2

TTF1

Islet-1

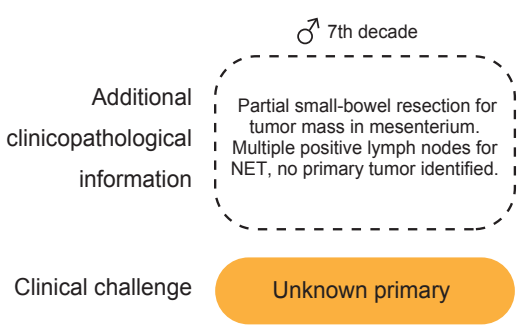

B

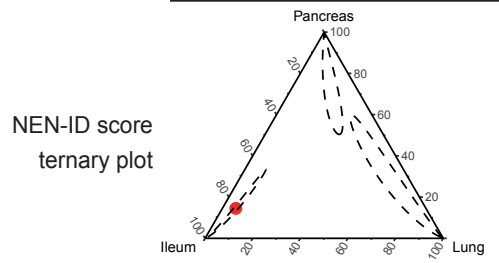

Additional Information

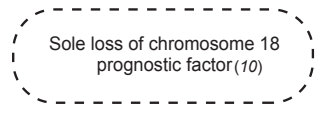

Calibrated diagnosis

\section{NET of Unknown Primary 1}

Grade 1 NET

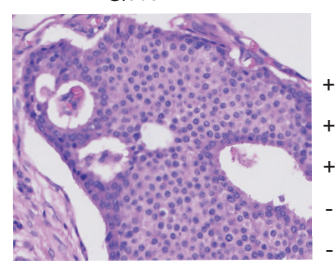

$\pi 7$ th decade

I Partial small-bowel resection for mor mass in mesenterium. I NET, no primary tumor identified.

\section{Unknown primary}

Small-intestinal NET

\section{Current practice}

NET of Unknown Primary 2

Grade 2 NET

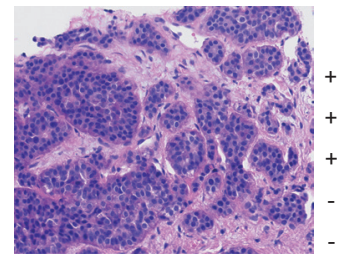

$\sigma^{\pi} 7$ th decade

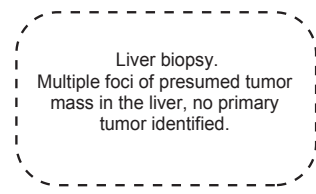

Unknown primary
MEN1 patient

Grade 2/3 NET

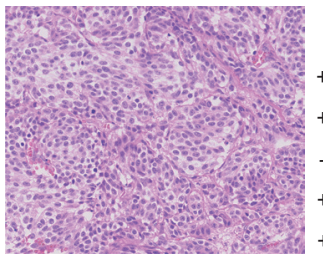

$\sigma^{\pi} 4$ th decade

- - - - - - - - - - - - Previous total pancreatectomy
for PanNETs. Left upper VATS lobectomy. Multiple positive lymph nodes. Secondary pathogenic MEN1 and likely pathogenic PIK3CA mutation confirmed.

- TP53 and RB1 WT

Pancreatic/ pulmonary origin

NEN-ID
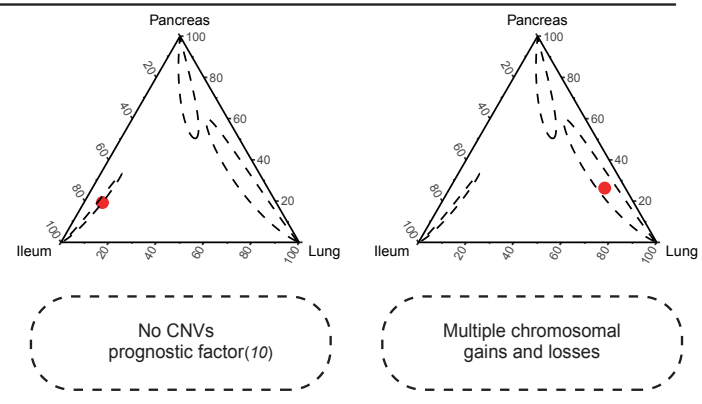

Small-intestinal NET

Pulmonary NET

Figure 2. Highlighted NETs of unknown primary from local test cohort. A, current practice in the UMCU to determine NET origin. Three confirmed NET cases (morphology, positive chromogranin A/synaptophysin) of different grades for which the origin was unknown or uncertain. NET origin cannot be determined by studying only morphology (H\&E slides). Additional immunohistochemical stainings (CDX2, TTF1, Islet-1, not shown) and clinicopathological information can make one or another origin more likely. B, NEN-ID classifier readily classifies cases. Probability scores fall within class tolerance ellipses. In addition, methylation-based copy number plots (not shown) can be used to further unravel tumorigenic mechanisms and provide prognostic information. 
Two NETs of unknown primary were mesenterial lymph node and liver metastases from sporadic NET patients. Samples were CDX2 positive and showed a nested growth pattern, suggesting small-intestinal origin, but this can also be seen in some PanNETs. The third case was a G2/3 NET resected from left upper lung lobe in a MEN1 patient (33), for which doubt could exist about primary pulmonary origin. The case was TTF1 and Islet-1 positive, and had a PIK3CA mutation. As TTF1 can be positive in PanNETs (especially high-grade neuroendocrine carcinomas) and mTOR mutations are more commonly seen in PanNETs $(15,34,35)$, it was uncertain if the lung lesion was a primary pulmonary NET or a pulmonary PanNET metastasis. The NEN-ID classifier readily confirmed small intestinal origin in the first two cases and classified the MEN1 NET as primary pulmonary NET. These predictions improved the current practice diagnostic workup by either confirming or narrowing down the differential diagnosis to a single site of origin. In addition, copy number profiling which can be derived from DNA methylation data showed various clinically relevant gains and losses.

\section{NEN-ID performance in external data and overall diagnostic accuracy}

To learn more about the predictive behavior of NEN-ID classifier, we compiled a "external test cohort" with all other publicly available 450k/EPIC methylation data of well-differentiated NETs of small-intestinal, pulmonary and pancreatic origin (10, 12-15). The external test cohort consisted of 59 sporadic small-intestinal NETs (primary tumors and metastases), 56 pulmonary NETs, 55 pancreatic NETs (sporadic, VHL and MEN1 syndrome associated), and 2 NETs of unknown primary (Table 1, Fig. S1). Predictive accuracy in the external test cohort was $96 \%$ (Fig. S4, Fig. S3D, Supplementary data 3 \& 4). The presence of hereditary pancreatic NET syndromes did not seem to affect accuracy, as 97\% (33 out of $10 \mathrm{VHL}+$ 24 MEN1 tumors) were correctly classified. To calculate overall sensitivity and specificity per origin, the local and external test cohorts were combined ( $n=193$, Table 4). Overall diagnostic accuracy was $96 \%$. Importantly $27 / 28$ metastatic samples in the combined test cohort were correctly classified, showing that methylation profiles in metastases remain highly specific for their origin. In addition, two patients in our local test cohort had matched

Table 4. Sensitivity and specificity in the combined local and external test cohort

\begin{tabular}{llllll}
\hline & $\begin{array}{l}\text { NEN-ID predicted } \\
\text { Small-intestinal }\end{array}$ & $\begin{array}{l}\text { NEN-ID predicted } \\
\text { Pulmonary }\end{array}$ & $\begin{array}{l}\text { NEN-ID predicted } \\
\text { Pancreatic }\end{array}$ & Sensitivity & Specificity \\
\hline Small-intestinal origin & 59 & 0 & 4 & $94 \%(59 / 63)$ & $99 \%(130 / 131)$ \\
Pulmonary origin & 0 & 65 & 1 & $98 \%(65 / 66)$ & $99 \%(127 / 128)$ \\
Pancreatic origin & 1 & 1 & 62 & $97 \%(62 / 64)$ & $96 \%(129 / 134)$ \\
\hline
\end{tabular}


primary and metastatic tumor samples. Highly correlated beta values and random forest classifier scores also suggested similar methylation in these cases (Fig. S5).

Last, we tested the behavior of the NEN-ID classifier on samples which were not part of the training cohort, including 28 NETs of other origins (stomach, duodenum, colon, appendix), 320 non-NET tumor samples and 180 normal samples from the TCGA database. A random forest model is built so that it will always produce 3 class votes adding up to $100 \%$. However, we observed the scores of the highest-scoring class were significantly lower in the NETs of other origins, TCGA tumor/normal cases compared to NET cases in the combined local and external test cohort (Fig. S6). Most non-NET cases were classified as pancreatic NET, although with lower probability scores, which might be a reflection of the methylation heterogeneity observed between pancreatic samples (Fig 1A \& C, Fig. S7), causing the ridge regression model to favor the pancreatic class in case raw random forest scores are equal for all classes.

\section{Erroneous data do not affect accuracy}

All probes used by the random forest model must be present for the random forest model to work. Consequently, failed probes are imputed which can introduce noise. We therefore tested the effect of failed probes on the NEN-ID model metrics by imputing random beta-values for increasing numbers of randomly selected measurement points (Fig. 1D). A percentage of probes was randomly imputed in the local test cohort. Surprisingly, up to $50 \%$ of all probes could be replaced with random beta values without drastic effect on prediction accuracy (Fig. S8A). With an increasing percentage of imputed probes, an increase of log loss was seen for the raw and calibrated random forest scores.

\section{Simulated normal cell contamination does not affect accuracy}

Similar to noise created by failed probes, a mix of tumor cells and non-tumor cells could obscure the methylation signature as recognized by the random forest model. In the clinical setting, biopsies and cytology specimens of NET metastases of unknown primary are often taken from lymph nodes, liver or lung. Contamination with lymphocytes or organ tissue could potentially harm predictive accuracy more than randomly imputed data. Using publicly available data of tissues and cell types, we determined if a pure population or a mix of normal cells might affect predictive accuracy (Fig. 1D). Reassuringly, accuracy remained perfect up to $25 \%$ simulated contamination and high (>90\% correct) up to $40 \%$ contamination (Fig. S8B). 


\section{The random forest model is available for further validation}

As validation with more cases from the scientific community is necessary, we set out to develop a tool that can be used without any experience in programming. The Shiny web application Methedrine was created based on CrystalMeth package (Vignette and source code Github cgeisenberger/crystalmeth) to provide a user-friendly web-based interface for data analysis. After uploading raw Illumina methylation array output (IDAT) files, Methedrine preprocesses data, analyzes tumor purity by the Purify package (36), plots copy numbers using the Conumee package (37) and predicts class for all uploaded samples. Optionally, output for three class problems can be plotted in a ternary plot (Fig. 1C). An example of the website output is provided in Fig. S9, showing a PanNET from our own institution with chromosome 11 loss (note copy number plot). The tool is compatible with both 450k and EPIC (850k) Illumina arrays, with a maximum of 10 tumor samples per run, and is available through www.nen-id.com or www.nen-id.net.

\section{DISCUSSION}

Whole genome methylation data-based predictive models are a trending topic in molecular tumor classification research and are revolutionizing cancer diagnostics. Here, we demonstrate - as a proof of concept - that a calibrated methylation-based random forest model can be used to accurately distinguish small-intestinal, pulmonary and pancreatic origin of primary and metastatic NETs. This application of a methylation-based model to predict origin has the potential to improve current diagnostic workup of NETs of unknown primary. As the necessity of basic programming experience to use the predictive model may delay further application, we present the easy-to-use web application Methedrine to analyze and classify new samples online, removing any barrier for further validation and clinical implementation.

In the diagnostic workup for patients with a NET metastasis of unknown primary, internal medicine specialists, (nuclear) radiologists and pathologists work together to determine the site of origin. However, if the primary site remains unknown based on imaging, the pathologist can try to make an educated guess based on tissue morphology and presence of markers like CDX2, TTF1, Islet-1, PAX-6/8, PDX1, SATB2, ATRX, DAXX, and ALT (29, 30, 38-41). Depending on the panel, and antibodies used in the panel, sensitivity and specificity ranges from $40 \%$ to $90 \%$. Overall diagnostic accuracy is reported to be up to $80 \%(29,30)$, which is similar to the diagnostic accuracy we observed in our local test cohort using a 
panel of CDX2, TTF1 and Islet-1. In comparison, our calibrated random forest prediction model shows superior classification accuracy. During repeated three-fold cross validation our predictive model showed near perfect sensitivity and specificity as demonstrated by multiclass ROC area under the curves $>0.99$. Accuracy of the final NEN-ID classifier for predicting small-intestinal, pulmonary and pancreatic origin was 96\% (187/193) on the combined local and external test cohort. Importantly, 27/28 metastases present in this combined test cohort were correctly classified, showing that methylation profiles remain origin-specific in the metastatic setting.

For a diagnostic test to be clinically applicable, it should meet high quality standards, be easy to perform and interpret, and work on any type/quality input material/data. It was previously shown that Illumina's methylation arrays are technically robust and have high inter-laboratory and inter-array correlations (8). As we provide a website for data analysis, there is no inter-observer variability. Furthermore, Illumina's methylation array can be performed on formalin-fixed biopsy specimens, making it useful in the diagnostic setting. Importantly, we demonstrated that the NEN-ID classifier accuracy is not significantly affected by simulated low quality samples. For up to $50 \%$ of simulated failed probes (imperfect data quality) and up to $25 \%$ simulated contamination (imperfect sample quality), the diagnostic accuracy remained highly accurate. In our experience, the number of failed probes stays well below $1 \%$, and as NETs are highly cellular and grow in solid/nested clumps, selecting or scraping $>80 \%$ tumor cells is within reach, also when growing in lymph nodes. This is illustrated by the fact that most tumors in the training cohort have low percentages of failed probes (Github whackeng/NEN-ID) and showed pathologist or data-driven estimates of tumor purity well above $80 \%(11,12)$. Although the true effect of normal cell contamination has to be determined in a non-simulated setting, this result indicates a certain degree of robustness of the predictive model for samples of low tumor purity.

While we show that the NEN-ID classifier is highly accurate in determining origin, a limitation is its restriction to predicting only classes present in the training cohort, which are well-differentiated NETs of small-intestinal, pulmonary or pancreatic origin. Sufficient data for NETs of other origins was not available, and including classes with few samples would have significantly decreased our training cohort size, as downsampling was used to create equal classes during probe selection and model building. The NEN-ID model is able to generate prediction scores on non-NET samples (like colon adenocarcinoma or normal skin), however prediction scores tend to be low (Fig. S6). This emphasizes the key role of the 
pathologist in confirming neuroendocrine tumor differentiation with neuroendocrine and keratin markers beforehand. The fact that the model is not trained to recognize NETs from other origins is also a challenge. However, studies show that NET metastases of unknown primary are - when the primary site is ultimately detected - most likely (>90\%) of smallintestinal, pancreatic and pulmonary origin (42-44). Indeed, these three NET subtypes have the highest proportion of distant metastases at presentation and are the most common, making them most relevant when studying NETs of unknown primary (1). Furthermore, studies using immunohistochemical panels to predict NET origin are often similarly limited to a selection of NET origins $(29,30,39-41)$. Nevertheless, when using the current NEN-ID model, the possibility of other origins should be considered. In the future, this tool needs to be expanded by including more classes, including rare origins and subtypes of NETs.

A strong point of our study is that the results are unbiased and reproducible. Many studies reporting the diagnostic value of transcription factors for certain origins (e.g., TTF1) do not report blinding for origin, and use different antibodies and staining protocols. Furthermore, as this is a non-commercial effort, there are no restrictions in using and building on the presented results by the scientific community, and there is no need in ordering specific arrays if the methylation array is available. The relative cost of Illumina's methylation array is less than commercial tests like CancerTYPE ID but more expensive than immunohistochemistry (43). However, it combines improved diagnostic accuracy with a wealth of additional information including copy number profiling and single gene methylation (e.g., CDKN2A methylation).

In conclusion, this study shows how combining publicly available data can result in a meaningful predictive model that predicts small-intestinal, pulmonary, and pancreatic NET origin with high accuracy. We present the complete source code on Github for others to investigate, reproduce and extend. As illustrated by three cases from our institution, the NEN-ID classifier can already be complementary in the clinical diagnostic workup of NET cases of unknown primary origin, e.g., when pancreatic and small-intestinal origin needs to be distinguished for diagnostic or therapeutic purposes. However, prospective studies need to be performed to determine the true added effect of this predictive model value for NETs of unknown primary. The web application enables straightforward clinical validation and implementation. By sharing, improving, and comparing data with the scientific community - we hope to fully harvest the potential of methylation-based prediction models, and ultimately enable prediction in all neuroendocrine neoplasms with high accuracy. 


\section{ACKNOWLEDGEMENTS}

We thank Aaron Isaacs for his introduction into methylation data-analysis. We thank Paul van Diest for his generous support and Erwin van der Biezen for supervising the processing of samples. We would like to thank all research teams for sharing their data, including the lungNENomics project and the Rare Cancers Genomics initiative. The results here are in part based upon data generated by the TCGA Research Network: https://www.cancer. gov/tcga. This work was supported by the Dutch Digestive Foundation/Maag Lever Darm Stichting (grant number CDG 14-020, L.A.A. Brosens, W.M. Hackeng).

\section{CONFLICT OF INTEREST:}

None

\section{REFERENCES:}

1. Man D, Wu J, Shen Z, Zhu X. Prognosis of patients with neuroendocrine tumor: a SEER database analysis. Cancer Manag Res. 2018;10:5629-38.

2. Scoazec JY, Couvelard A, Monges G, Guyetant S, Bisot-Locard S, Parot X, et al. Professional Practices and Diagnostic Issues in Neuroendocrine Tumour Pathology: Results of a Prospective One-Year Survey among French Pathologists (the PRONET Study). Neuroendocrinology. 2017;105(1):67-76.

3. Chan DL, Clarke SJ, Diakos Cl, Roach PJ, Bailey DL,

Singh S, et al. Prognostic and predictive biomarkers in neuroendocrine tumours. Crit Rev Oncol Hematol. 2017;113:268-82.

4. Herrera-Martinez AD, Hofland LJ, Galvez Moreno MA,

Castano JP, de Herder WW, Feelders RA. Neuroendocrine neop-

lasms: current and potential diagnostic, predictive and prognostic markers. Endocr Relat Cancer. 2019;26(3):R157-R79.

5. Pavel M, O'Toole D, Costa F, Capdevila J, Gross D, Kianmanesh R, et al. ENETS Consensus Guidelines Update for the Management of Distant Metastatic Disease of Intestinal, Pancreatic, Bronchial Neuroendocrine Neoplasms (NEN) and NEN of Unknown Primary Site. Neuroendocrinology. 2016;103(2):172-85. 6. Lokk K, Modhukur V, Rajashekar B, Martens K, Magi R, Kolde R, et al. DNA methylome profiling of human tissues identifies global and tissue-specific methylation patterns. Genome Biol. 2014;15(4):r54.

$7 . \quad$ Moran S, Martinez-Cardus A, Sayols S, Musulen E, Balana C, Estival-Gonzalez A, et al. Epigenetic profiling to classify cancer of unknown primary: a multicentre, retrospective analysis. Lancet Oncol. 2016;17(10):1386-95.

8. Capper D, Jones DTW, Sill M, Hovestadt V, Schrimpf $D$, Sturm D, et al. DNA methylation-based classification of central nervous system tumours. Nature. 2018;555(7697):469-74.

9. Orozco JIJ, Knijnenburg TA, Manughian-Peter AO,

Salomon MP, Barkhoudarian G, Jalas JR, et al. Epigenetic profiling for the molecular classification of metastatic brain tumors. Nat
Commun. 2018;9(1):4627

10. Karpathakis A, Dibra H, Pipinikas C, Feber A, Morris T, Francis J, et al. Prognostic Impact of Novel Molecular Subtypes of Small Intestinal Neuroendocrine Tumor. Clin Cancer Res. 2016;22(1):250-8.

11. Chan CS, Laddha SV, Lewis PW, Koletsky MS, Robzyk K, Da Silva E, et al. ATRX, DAXX or MEN1 mutant pancreatic neuroendocrine tumors are a distinct alpha-cell signature subgroup. Nat Commun. 2018;9(1):4158.

12. Laddha SV, da Silva EM, Robzyk K, Untch BR, Ke $\mathrm{H}$, Rekhtman N, et al. Integrative Genomic Characterization Identifies Molecular Subtypes of Lung Carcinoids. Cancer Res. 2019;79(17):4339-47.

13. Timp W, Bravo HC, McDonald OG, Goggins M, Umbricht C, Zeiger M, et al. Large hypomethylated blocks as a universal defining epigenetic alteration in human solid tumors. Genome Med. 2014;6(8):61.

14. Tirosh A, Killian JK, Petersen D, Zhu YJ, Walker RL, Blau JE, et al. Distinct DNA Methylation Signatures in Neuroendocrine Tumors Specific for Primary Site and Inherited Predisposition. J Clin Endocrinol Metab. 2020;105(10).

15. Alcala N, Leblay N, Gabriel AAG, Mangiante L, Hervas $D$, Giffon T, et al. Integrative and comparative genomic analyses identify clinically relevant pulmonary carcinoid groups and unveil the supra-carcinoids. Nat Commun. 2019;10(1):3407.

16. Mill J, Hannon E, Burrage J. DNA methylation profiles of Purified Blood Cell types. In: Omnibus GE, editor. 2018.

17. R Core Team. R: A language and environment for statistical computing. URL: https://www.R-project.org/. Vienna, Austria: R Foundation for Statistical Computing; 2017.

18. Tian Y, Morris TJ, Webster AP, Yang Z, Beck S, Feber A, et al. ChAMP: updated methylation analysis pipeline for Illumina BeadChips. Bioinformatics. 2017;33(24):3982-4.

19. van der Maaten L, Hinton G. Visualizing data using 
t-SNE. Journal of Machine Learning Research. 2008;9:2579-605. 20. Breiman L. Random forests. Mach Learn. 2001;45(1):5-32

21. Hand DJ, Till RJ. A simple generalisation of the area under the ROC curve for multiple class classification problems. Mach Learn. 2001;45(2):171-86.

22. Hamilton NE, Ferry M. ggtern: Ternary Diagrams Using ggplot2. J Stat Softw. 2018;87(Cn3):1-17.

23. Varmuza K, Filzmoser P. Introduction to multivariate statistical analysis in chemometrics: CRC press; 2016.

24. Tedla N, Dwyer J, Truskett P, Taub D, Wakefield D,

Lloyd A. Phenotypic and functional characterization of lymphocytes derived from normal and HIV-1-infected human lymph nodes. Clin Exp Immunol. 1999;117(1):92-9.

25. Battaglia A, Ferrandina G, Buzzonetti A, Malinconico $P$, Legge $F$, Salutari $V$, et al. Lymphocyte populations in human lymph nodes. Alterations in CD4+CD25+ T regulatory cell phenotype and T-cell receptor Vbeta repertoire. Immunology. 2003;110(3):304-12.

26. Setiadi AF, Ray NC, Kohrt HE, Kapelner A, Carcamo-Cavazos V, Levic EB, et al. Quantitative, architectural analysis of immune cell subsets in tumor-draining lymph nodes from breast cancer patients and healthy lymph nodes. PLoS One. 2010;5(8):e12420

27. Stankovic B, Bjorhovde HAK, Skarshaug R, Aamodt $H$, Frafjord A, Muller E, et al. Immune Cell Composition in Human Non-small Cell Lung Cancer. Front Immunol. 2018;9:3101.

28. Maros ME, Capper D, Jones DTW, Hovestadt V, von Deimling A, Pfister SM, et al. Machine learning workflows to estimate class probabilities for precision cancer diagnostics on DNA methylation microarray data. Nat Protoc. 2020;15(2):479-512.

29. Koo J, Mertens RB, Mirocha JM, Wang HL, Dhall D.

Value of Islet 1 and PAX8 in identifying metastatic neuroendocrine tumors of pancreatic origin. Mod Pathol. 2012;25(6):893-901. 30. Yang Z, Klimstra DS, Hruban RH, Tang LH. Immunohistochemical Characterization of the Origins of Metastatic Well-differentiated Neuroendocrine Tumors to the Liver. Am J Surg Pathol. 2017;41(7):915-22.

31. Soga J, Tazawa K. Pathologic analysis of carcinoids. Histologic reevaluation of 62 cases. Cancer. 1971;28(4):990-8.

32. Bellizzi AM. Immunohistochemistry in the diagnosis and classification of neuroendocrine neoplasms: what can brown do for you? Hum Pathol. 2020;96:8-33.

33. Van Den Broek MFM, De Laat JM, Van Leeuwaarde RS, Van De Ven AC, De Herder WW, Dekkers OM, et al. The management of neuroendocrine tumors of the lung in MEN1: results from the Dutch MEN1 Study Group. J Clin Endocrinol Metab. 2020.

34. Scarpa A, Chang DK, Nones K, Corbo V, Patch A-M, Bailey $P$, et al. Whole-genome landscape of pancreatic neuroendocrine tumours. Nature. 2017;543(7643):65-71.

35. Simbolo M, Mafficini A, Sikora KO, Fassan M, Barbi S, Corbo $V$, et al. Lung neuroendocrine tumours: deep sequencing of the four World Health Organization histotypes reveals chromatin-remodelling genes as major players and a prognostic role for TERT, RB1, MEN1 and KMT2D. J Pathol. 2017;241(4):488-500. 36. Johann PD, Jager N, Pfister SM, Sill M. RF_Purify: a novel tool for comprehensive analysis of tumor-purity in methylation array data based on random forest regression. BMC Bioinformatics. 2019;20(1):428.

37. Hovestadt V, Zapatka M. Conumee: Enhanced copy-number variation analysis using Illumina DNA methylation arrays. R package version 1.9.0, http://bioconductor.org/packages/conumee/.

38. Dogeas E, Karagkounis G, Heaphy CM, Hirose K, Pawlik TM, Wolfgang $C L$, et al. Alternative lengthening of telomeres predicts site of origin in neuroendocrine tumor liver metastases. J Am Coll Surg. 2014;218(4):628-35.

39. Zhao LH, Chen C, Mao CY, Xiao H, Fu P, Xiao HL, et al. Value of SATB2, ISL1, and TTF1 to differentiate rectal from other gastrointestinal and lung well-differentiated neuroendocrine tumors. Pathol Res Pract. 2019;215(7):152448.

40. Yang MX, Coates RF, Ambaye A, Cortright V, Mitchell JM, Buskey AM, et al. NKX2.2, PDX-1 and CDX-2 as potential biomarkers to differentiate well-differentiated neuroendocrine tumors. Biomark Res. 2018;6:15.

41. Tseng IC, Yeh MM, Yang CY, Jeng YM. NKX 6-1 Is a Novel Immunohistochemical Marker for Pancreatic and Duodenal Neuroendocrine Tumors. Am J Surg Pathol. 2015;39(6):850-7. 42. Massimino KP, Han E, Pommier SJ, Pommier RF. Laparoscopic surgical exploration is an effective strategy for locating occult primary neuroendocrine tumors. Am J Surg. 2012;203(5):628-31.

43. Chauhan A, Farooqui Z, Silva SR, Murray A, Hodges $K B, Y u Q$, et al. Integrating a 92-Gene Expression Analysis for the Management of Neuroendocrine Tumors of Unknown Primary. Asian Pac J Cancer Prev. 2019;20(1):113-6.

44. De Dosso S, Treglia G, Pascale M, Tamburello A, Santhanam P, Kroiss AS, et al. Detection rate of unknown primary tumour by using somatostatin receptor PET/CT in patients with metastatic neuroendocrine tumours: a meta-analysis. Endocrine. 2019;64(3):456-68. 


\section{SUPPLEMENTARY FIGURES}
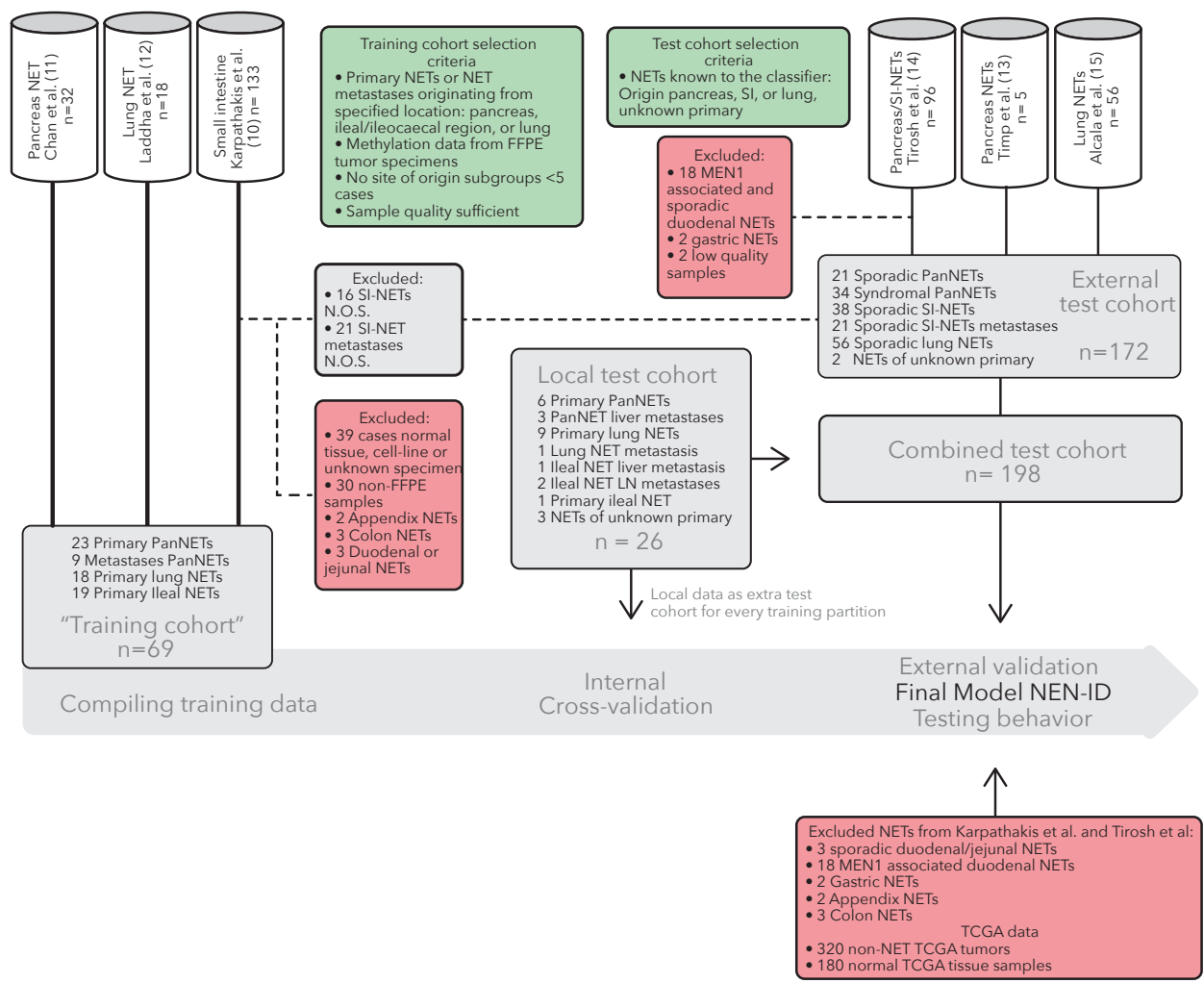

Supplementary figure 1. Overview of the training and test cohort selection procedure by selection criteria. Abbreviations: SI-NET, small-intestinal NET; PanNET, pancreatic NET; LN, lymph node. 

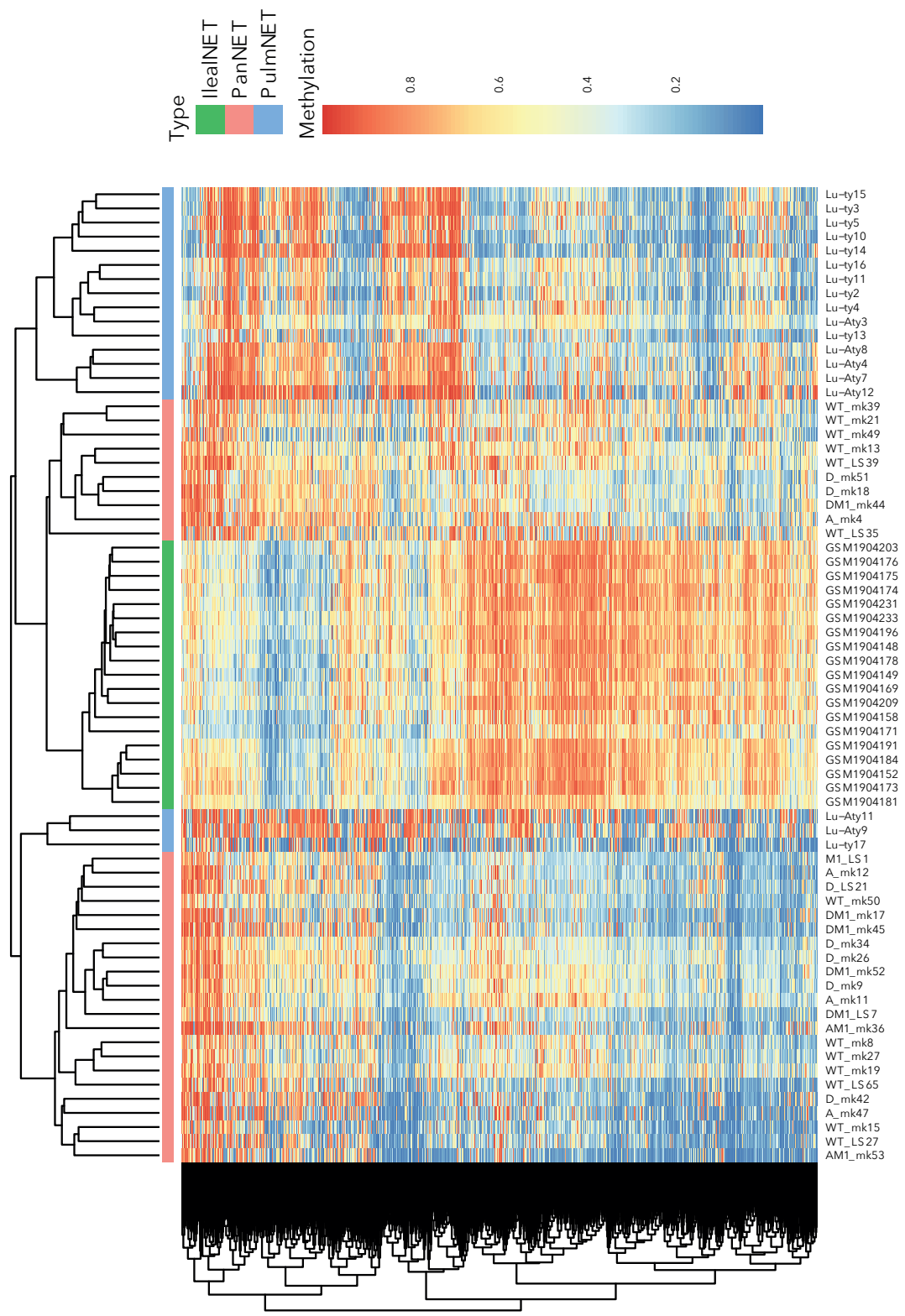

Supplementary figure 2. Unsupervised hierarchical clustering with euclidean distance of the entire training cohort. 3 pulmonary NETs cluster with the pancreatic NETs (lu-ty17, lu-Aty9, lu-Aty11), and represent the Lung Carcinoid Type (LC2) carcinoids. 
A
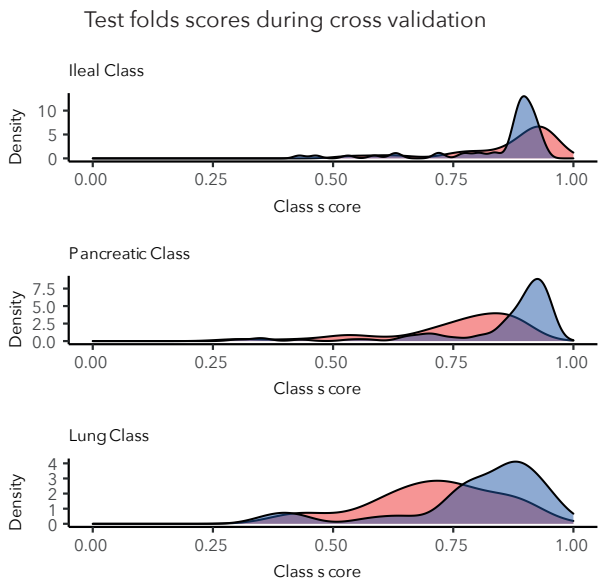

C

Scores NEN-ID model for local test cohort
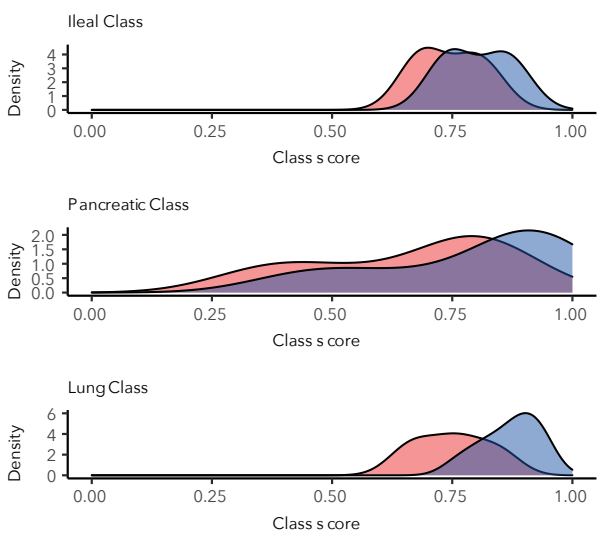

B

Out of bag scores final model
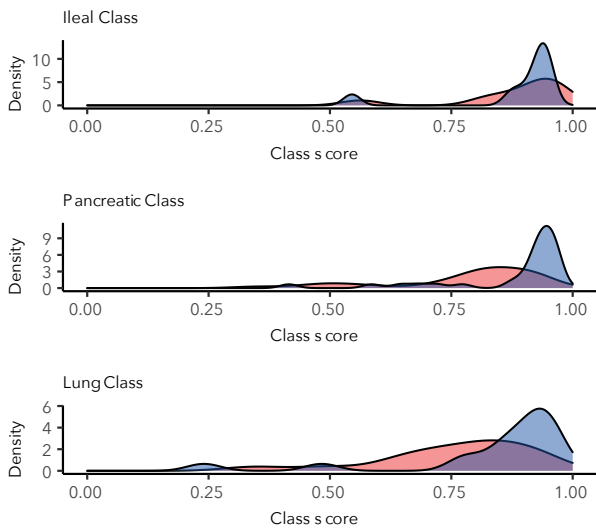

D Scores NEN-ID model for external test cohort
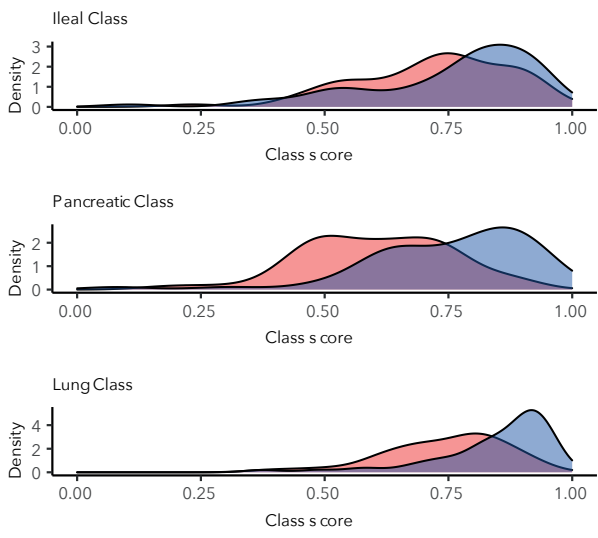

Model fit

Raw RF

Calibrated RF

Supplementary figure 3. A, density plot of raw and calibrated predicted scores of cases in the entire training cohort when present in "test-folds" during $3 \times 3$ cross validation. B, density plot of raw and calibrated out of bag (OOB) scores of the random forest model (NEN-ID) trained on the entire training cohort. C and D, density plots of raw and calibrated scores of NEN-ID classifier for the local and external test cohort, respectively. Ridge regression calibration increases correct class scores, while decreasing incorrect class scores, and this effect is well seen during cross

validation. For all origins, most true scores are $>80 \%$. Nevertheless, cases with lower true class scores are present, as is illustrated by the lower parts of the density plots. 


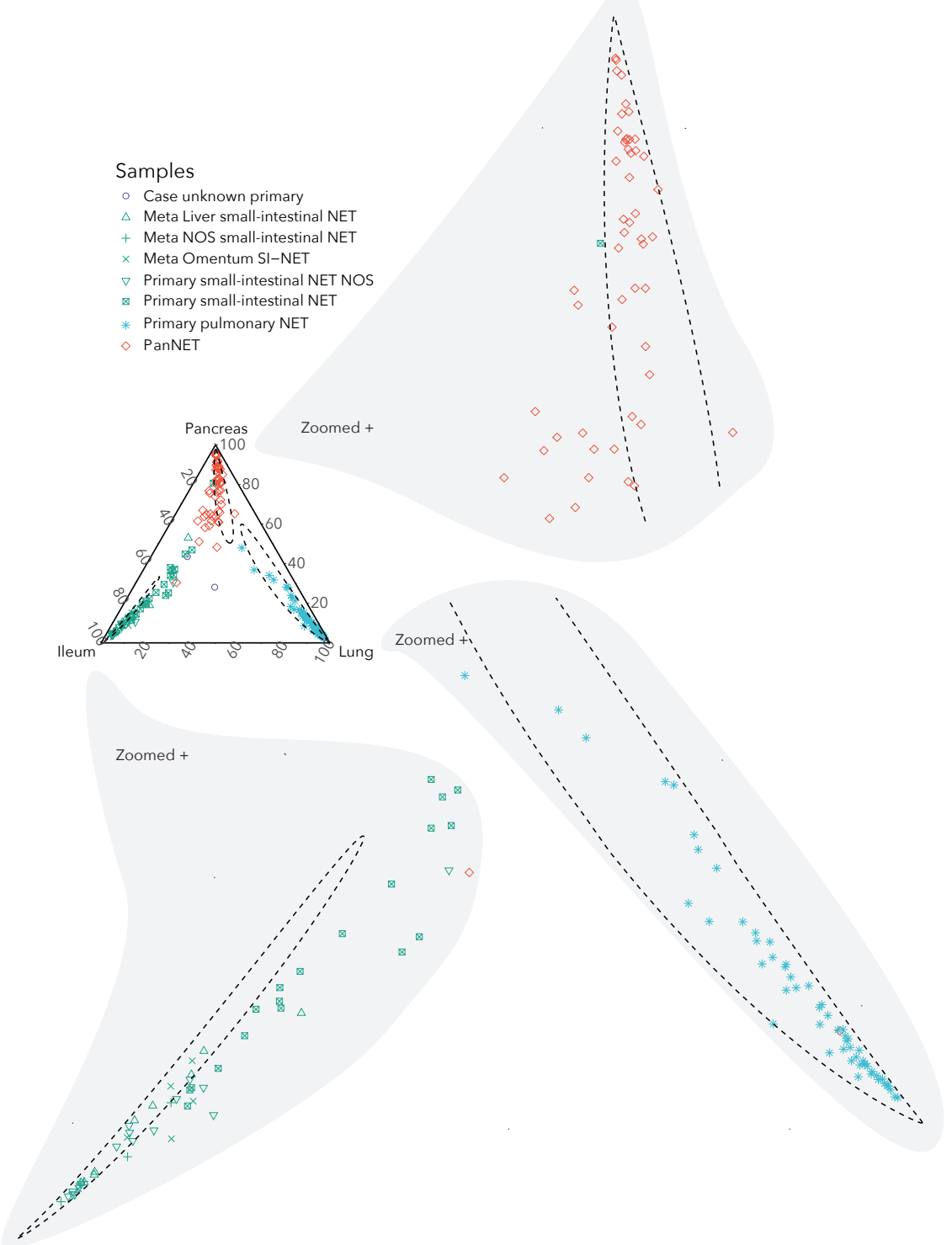

Supplementary figure 4. Calibrated scores of the external test cohort in a ternary plot. Zoomed clouds of crouded areas near the tolerance ellipse of small intestinal, pulmonary and pancreatic origin. 
A

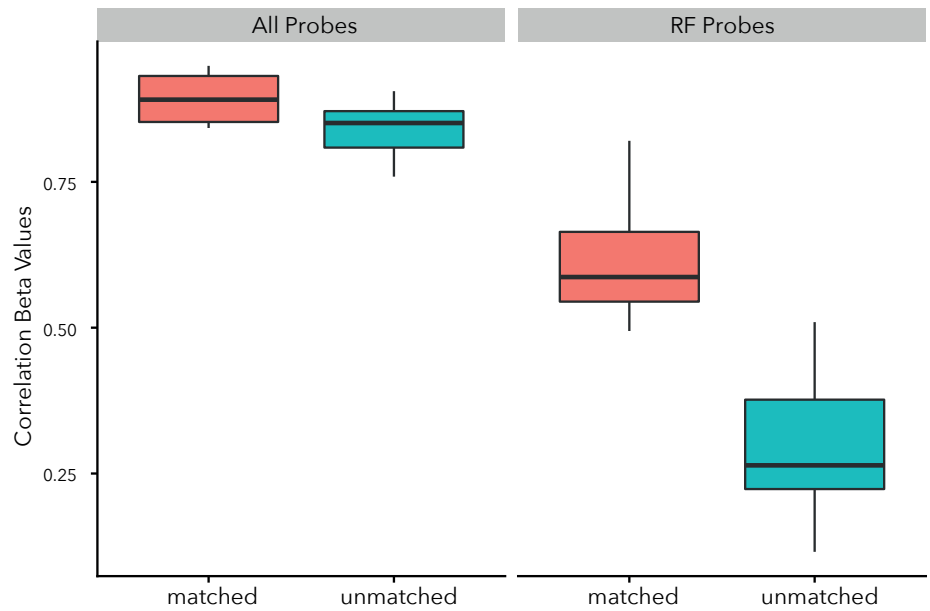

B

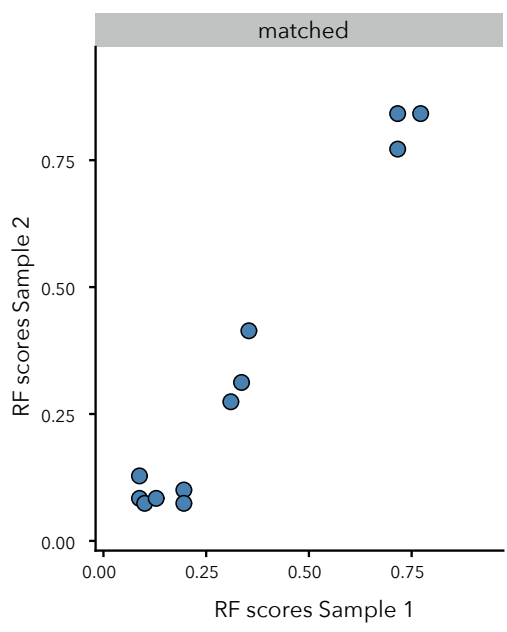

Supplementary figure 5. A. Methylation profiles were compared for paired and unpaired cases. In this analysis, higher correlation values correspond to more similar methylation patterns. Paired cases (i.e. primary and metastasis) showed higher correlation values, both when taking into account all available probes (left panel) or only those used in the classifier (right panel). B extends this analysis to the random forest output. Classifier scores are highly similar for paired samples with a correlation of $R=0.99$. These analyses suggest that tumors from different locations in the same patient are alike to each other in terms of their methylation profiles and classification results. 


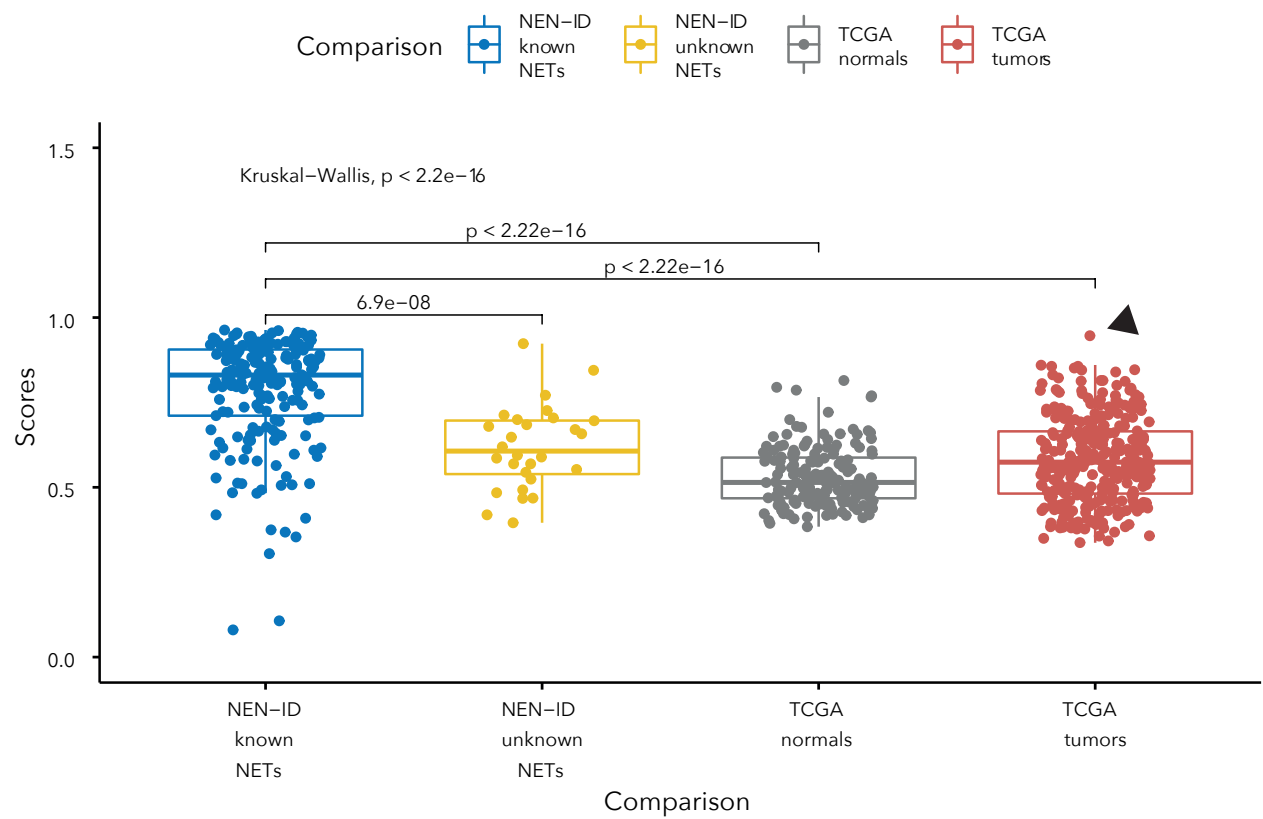

Supplementary figure 6. Comparison of the NEN-ID scores in the combined local and external test cohort "NENID known NETs", samples of NETs that the NEN-ID model was not trained to recognize ("NEN-ID unknown NETs", consisting of gastric, duodenal, appendix and colon samples), "TCGA normals" tissue (22 types), and non-NET "TCGA tumors" tissue (32 types). The NEN-ID classifier gives a three-score output (small-intestine, lung and pancreas), and the highest score is used to call the class. The scores of the majority class are given for the groups "NEN-ID unknown NETs", "TCGA normals" and "TCGA tumors", and are therefor per definition above 0.33. In contrast for "NEN-ID known NETs", the scores for the true class of origin were given, and not scores assocated with the majority class, making it a more stringent comparison. This means few cases score less than 0.33. Scores are significantly higher in "NEN-ID known NETs" than other groups. Of note, the case with highest scores in "TCGA tumors" (arrowhead) is annotated as PAAD (pancreatic ductal adenocarcinoma) by TCGA, and was predicted by NEN-ID to be a pancreatic NET. A recent publication which revised all PAAD cases found this case was in fact a neuroendocrine tumor*.

*Nicolle R, Raffenne J, Paradis V, Couvelard A, de Reynies A, Blum Y, et al. Prognostic biomarkers in pancreatic cancer: avoiding errata when using the TCGA dataset. Cancers 2019, 11(1), 126. 
A

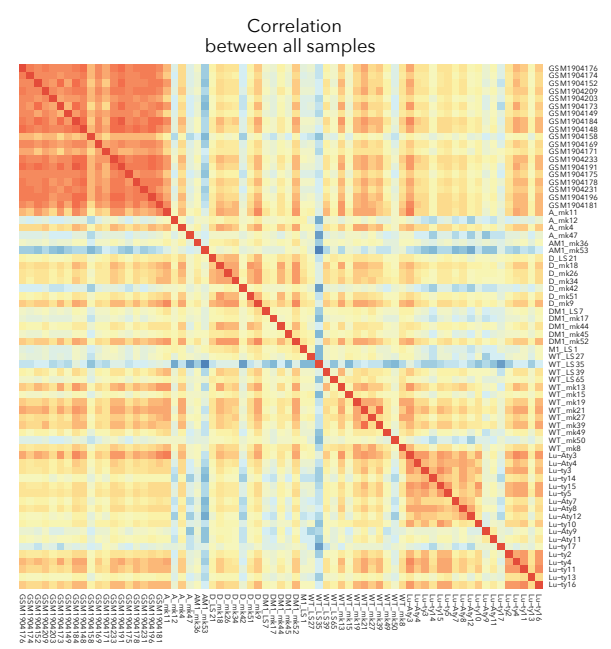

B

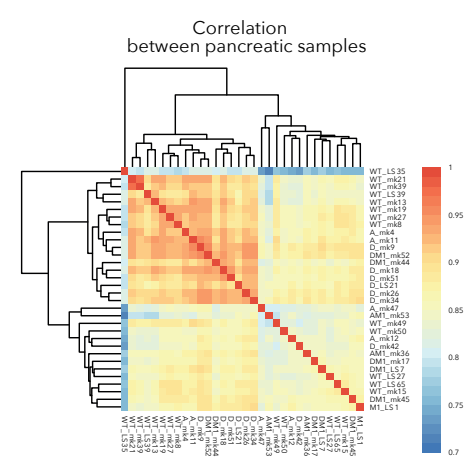

C

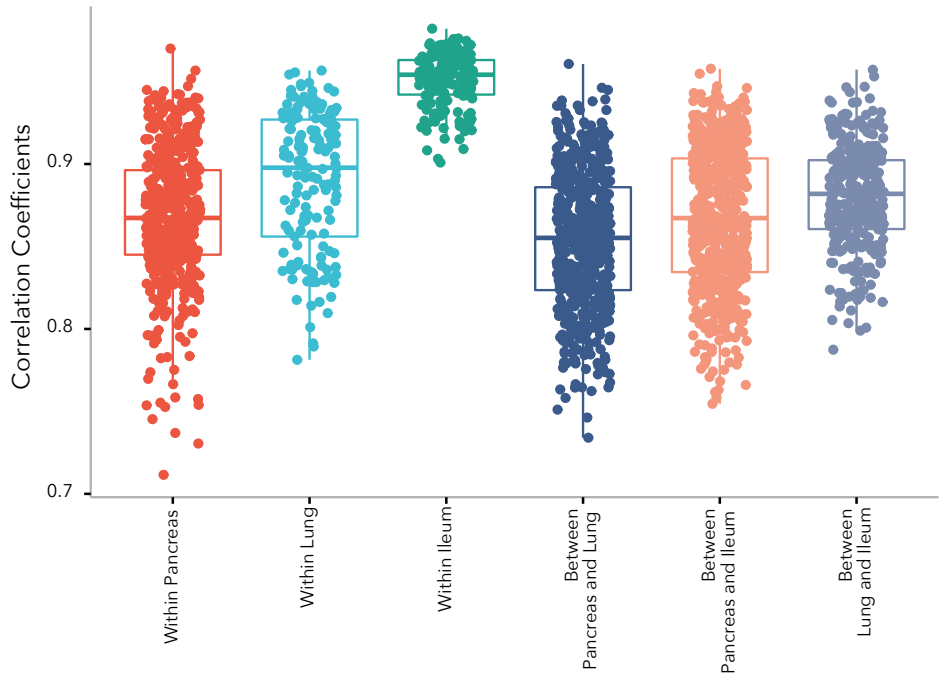

Supplementary figure 7. A, All beta value pearsons correlation between samples in the entire training cohort without clustering. B, pearsons correlation for pancreatic samples in the entire training cohort with clustering, showing that about half of the samples are less correlated with other pancreatic NETs, which could indicate methylation heterogeneity within pancreatic NETs. C, boxplots showing intra and inter class pearsons correlations. 
A

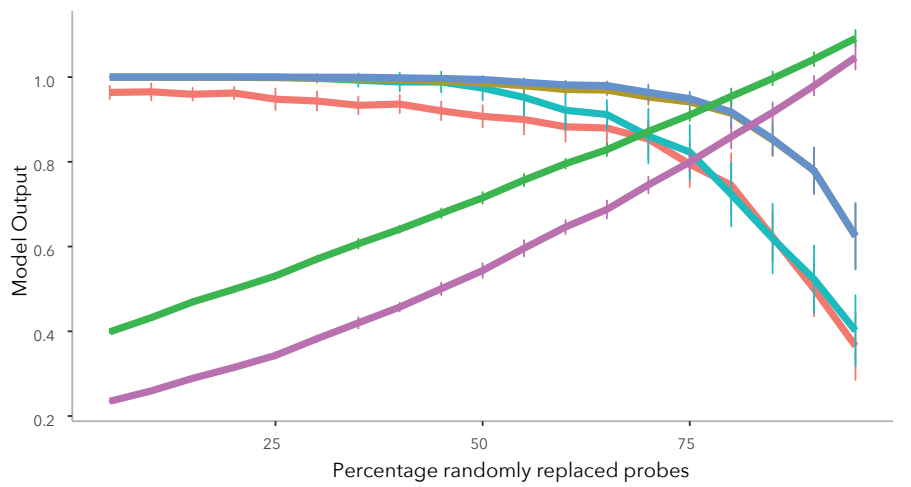

Model Metrics

- RF Mean Accuracy

- RF Mean AUC

- RF Mean LogLoss

- RF+LR Mean Accuracy

- RF+LR Mean AUC

- RF+LR Mean LogLoss

B

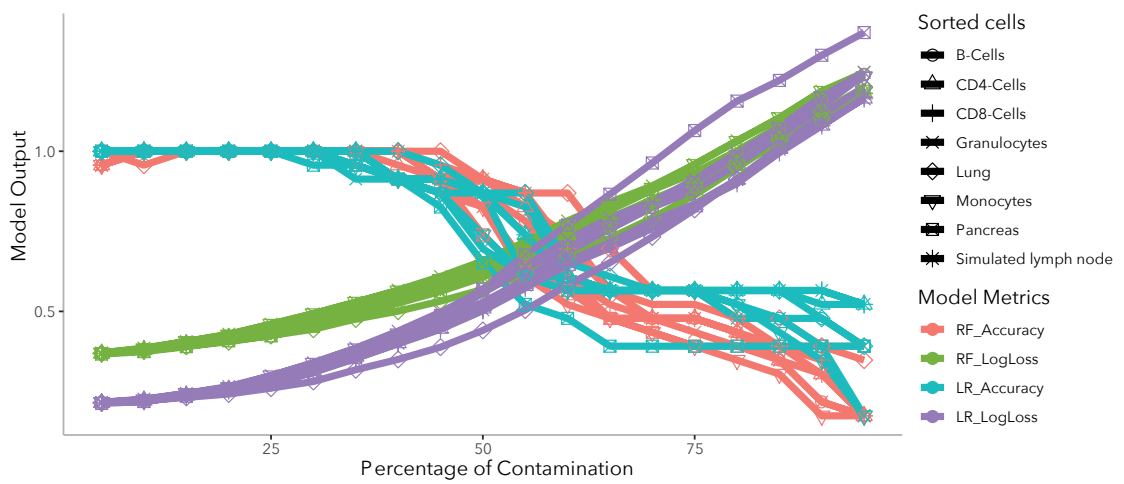

Supplementary figure 8. Effect of random probe imputation (A) and normal cell contamination (B) on the NEN-ID classifier accuracy. A, the mean model metrics of 30 repeats of random probe imputation are given, with standard deviation error bars. Mean predictive accuracy stays near perfect up to $50 \%$ simulated random probe imputation. $B$, predictive accuracy stays perfect up to up to $25 \%$ simulated normal cell contamination. Of note, we did not correct for the percentage failed probes or normal cells already inherently present in the samples. 


\section{NEN-ID Classification report}

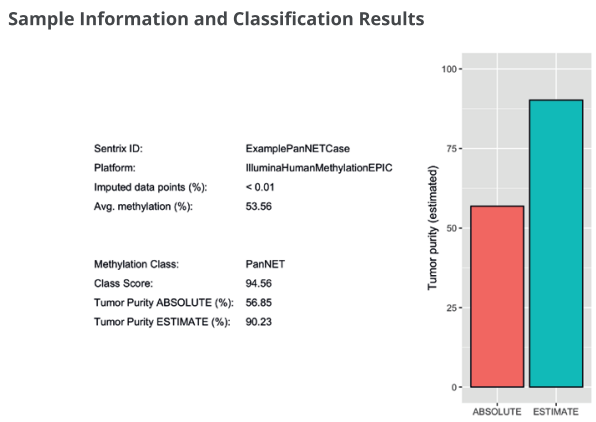

Ternary Plot

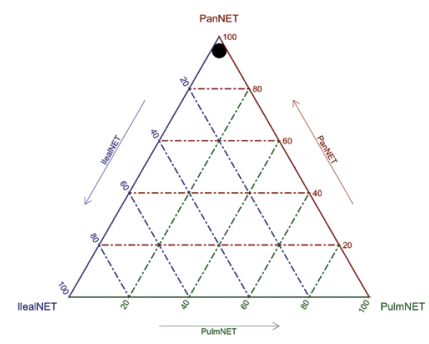

Copynumber Plot

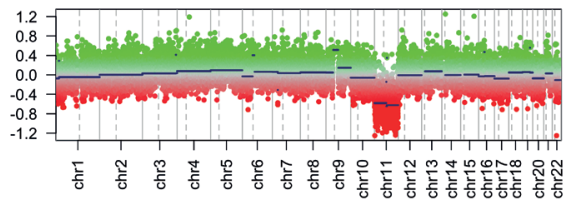

References \& Disclaimer

The NEN.ID classifcation algorithm has been published by Hackeng et al., 2020 (Jouma/Name, 2020). Tumor purity estimation is performed with RF_Purify (Johann et al, BMC Bioinformatics, 2039). Copy-number plots are based on the Conumee package published by Hovestadt \& Zapatka (avaliable on Bioconductor).

Note: NENID is not a diagnostic tool. Classifcation using methylation profling is a research tool under development, it is not venfed and has not been clinically validated. Implementation of the results in a clinical setting is in the sole responsibility of the treating physician. intended for non-commercial use only.

Report created: 2020-11-10 11:25:47

Supplementary figure 9. Website output for the NEN-ID classifier after uploading a case IDAT file (raw output Illumina methylation array) of a pancreatic NET. The percentage of imputed probes and estimated tumor purity are given. Classification ternary plots are shown and indicate pancreatic origin. A copy number plot (log2 ratio of sample probe intensities vs. controls) shows a clear decrease of chromosome 11 intensities, indicating loss of heterozygosity of chromosome 11 (locus MEN1). The other chromosomes do not show aberrant intensity ratios. 
Supplementary Table 1

\begin{tabular}{|c|c|c|c|c|}
\hline & Karpathakis et al. $(\mathrm{N}=19)$ & Laddha et al. $(\mathrm{N}=18)$ & Chan et al. $(\mathrm{N}=32)$ & Hackeng et al. $(\mathrm{N}=26)$ \\
\hline \multicolumn{5}{|l|}{$\overline{\mathrm{Age}}$} \\
\hline Mean (SD) & $59.3(13.0)$ & $60.9(16.8)$ & $52.5(11.3)$ & $58.7(11.9)$ \\
\hline Median [Min, Max] & $62.0[31.0,83.0]$ & $66.0[28.0,81.0]$ & $53.5[30.0,73.0]$ & $58.5[32.0,79.0]$ \\
\hline \multicolumn{5}{|l|}{ Gender } \\
\hline Male & $7(36.8 \%)$ & $4(22.2 \%)$ & $16(50.0 \%)$ & $17(65.4 \%)$ \\
\hline Female & $12(63.2 \%)$ & $14(77.8 \%)$ & $16(50.0 \%)$ & $9(34.6 \%)$ \\
\hline \multicolumn{5}{|l|}{ Origin } \\
\hline Ileum & $19(100 \%)$ & $0(0 \%)$ & $0(0 \%)$ & $4(15.4 \%)$ \\
\hline Lung & $0(0 \%)$ & $18(100 \%)$ & $0(0 \%)$ & $10(38.5 \%)$ \\
\hline Pancreas & $0(0 \%)$ & $0(0 \%)$ & $32(100 \%)$ & $9(34.6 \%)$ \\
\hline Unknown Primary & $0(0 \%)$ & $0(0 \%)$ & $0(0 \%)$ & $3(11.5 \%)$ \\
\hline \multicolumn{5}{|l|}{ Sample Site } \\
\hline Ileum & $9(47.4 \%)$ & $0(0 \%)$ & $0(0 \%)$ & $1(3.8 \%)$ \\
\hline Terminal ileum & $6(31.6 \%)$ & $0(0 \%)$ & $0(0 \%)$ & $0(0 \%)$ \\
\hline Ileocaecal & $4(21.1 \%)$ & $0(0 \%)$ & $0(0 \%)$ & $0(0 \%)$ \\
\hline Right Lower Lobe & $0(0 \%)$ & $3(16.7 \%)$ & $0(0 \%)$ & $1(3.8 \%)$ \\
\hline Right Middle Lobe & $0(0 \%)$ & $2(11.1 \%)$ & $0(0 \%)$ & $2(7.7 \%)$ \\
\hline Right Upper Lobe & $0(0 \%)$ & $1(5.6 \%)$ & $0(0 \%)$ & $1(3.8 \%)$ \\
\hline Left Lower Lobe & $0(0 \%)$ & $8(44.4 \%)$ & $0(0 \%)$ & $3(11.5 \%)$ \\
\hline Lingula & $0(0 \%)$ & $1(5.6 \%)$ & $0(0 \%)$ & $0(0 \%)$ \\
\hline Left Upper Lobe & $0(0 \%)$ & $3(16.7 \%)$ & $0(0 \%)$ & $3(11.5 \%)$ \\
\hline Pancreas & $0(0 \%)$ & $0(0 \%)$ & $23(71.9 \%)$ & $6(23.1 \%)$ \\
\hline Liver & $0(0 \%)$ & $0(0 \%)$ & $8(25.0 \%)$ & $5(19.2 \%)$ \\
\hline Lymph Node & $0(0 \%)$ & $0(0 \%)$ & $1(3.1 \%)$ & $4(15.4 \%)$ \\
\hline
\end{tabular}

SD, standard deviation 
CHAPTER

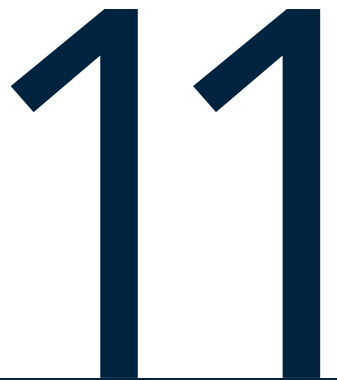

Summarizing discussion 
A dazzle* of neuroendocrine tumors: even though their individual behavior ranges from indolent to aggressive, and pathways travelled differ, all have a similar trabecular (striped) or nested morphology, making accurate histopathological tumor identification challenging. This thesis aims to provide a new perspective on neuroendocrine tumors by improving our fundamental understanding of the early tumorigenesis, and evaluate novel (epigenetic) means to accurately classify behavior and tissue of origin. 


\section{PART 1. CELL-DIFFERENTIATION SPECIFIC DUODENOPANCREATIC TUMORIGENIC PATHWAYS}

Cell-differentiation creates structural cell-type specific vulnerabilities for tumorigenic pathways. As reviewed in chapter 2, hotspot mutations in the oncogene KRAS play a central role in sporadic pancreatic ductal-cell tumorigenesis, and are seen in virtually all (>90\%) pancreatic ductal adenocarcinomas. Moreover, KRAS mutations are present in microscopic (usually $<5 \mathrm{~mm}$ ) areas of ductal dysplasia, called Pancreatic Intraepithelial Neoplasia (PanIN). For this reason, KRAS mutations are considered early - and initiating - events of pancreatic ductal-cell tumorigenesis. In contrast, KRAS mutations are never seen in the pancreatic neuroendocrine tumorigenesis. Instead, commonly mutated genes in sporadic pancreatic neuroendocrine tumors include the tumor suppressors MEN1 (40\%) and ATRX or DAXX (30\%) (1). Mutual exclusive mutations in ATRX or DAXX are associated with the alternative lengthening of telomeres phenotype (ALT) (2). Regularly, neuroendocrine proliferations/small tumors ( $<5 \mathrm{~mm}$ ) - also called microadenomas - are incidentally detected in pancreatic specimens resected for other disease (e.g., ductal adenocarcinoma). However, the significance of these microadenomas is debatable, as it is unclear if they are multiclonal and hyperplastic, or monoclonal and neoplastic (i.e. autonomous) (3). The presence of MEN1, ATRX and DAXX mutations or ALT in sporadic microadenomas would establish their role as neoplastic precursors, like KRAS mutations in PanINs. Importantly, if sporadic microadenomas appear to be neoplastic precursors, they could provide insight in the sequence of events in the sporadic neuroendocrine tumorigenesis. In chapter 3 , we show that Menin, the protein product of the MEN1 gene, is aberrantly expressed in $14 / 19$ (74\%) of sporadic microadenomas by immunohistochemistry. It is likely that at least some of these microadenomas harbor bi-allelic somatic MEN1 inactivation, as we show that loss of expression correlates with mutations in a series of pancreatic neuroendocrine tumors with sequencing data. Aberrant ATRX/DAXX expression or ALT was not seen. These results not only support the hypothesis that microadenomas are bona-fide neoplastic precursors, but also suggest that MEN1 alterations play a role in initiating sporadic pancreatic neuroendocrine tumorigenesis. In addition, the lack of ATRX/DAXX loss and ALT indicates they do not initiate tumorigenesis, and generally are later events in the sporadic neuroendocrine tumorigenesis. This is in line with studies reporting increased prevalence of ALT with tumor size, the regular finding of subclonal/heterogenous ATRX/DAXX loss, and the lack of microadenomas and pancreatic neuroendocrine tumors in patients with ATR-X syndrome (germline ATRX mutations) (4-6). Furthermore, these findings confirm the validity 
of MEN1-syndrome as model for the sporadic neuroendocrine tumorigenesis: all MEN1syndrome associated pancreatic microadenomas have loss of the wildtype MEN1 allele $(7,8)$. ATRX/DAXX loss and ALT are seen as late event in a subset $(6 \%)$ of MEN1-syndrome associated pancreatic neuroendocrine tumors $(9,10)$.

Whereas the pancreas in MEN1-syndrome is microscopically characterized by multifocal microadenomas and neuroendocrine tumors, as extensively characterized in a different chapter of this thesis (chapter 9), the glucagon-cell hyperplasia neoplasia syndrome (GCHN)-affected pancreas additionally shows a generalized pancreatic islet hyperplasia. In chapter 4, we summarize clinicopathological characteristics and the tumorigenic mechanism of GCHN, which is considered a true hyperplasia to neoplasia syndrome. Briefly, hyperaminoacidemia, resulting from impaired ureagenesis and gluconeogenesis in the liver due to mutations in the glucagon receptor, promotes alpha-cell (glucagon-cell) proliferation in the pancreatic islets (11). The proliferative activity drastically increases the chance of random genetic errors during replication (12), resulting in multiple autonomous pancreatic alpha-cell neuroendocrine tumors. In chapter 5 , we speculate that the mechanism of tumorigenesis in MEN1-syndrome associated gastrinomas may have similarities to GCHN. In contrast to MEN1-syndrome associated pancreatic neuroendocrine tumors, gastrinomas in MEN1-syndrome often retain the wildtype MEN1-allele. Furthermore, they are invariably surrounded by duodenal gastrin-cell (G-cell) hyperplasia. Almost all patients with MEN1syndrome develop parathyroid-hormone secreting parathyroid adenomas resulting in hypercalcemia. Similar to the amino acids in GCHN, we hypothesize that the extracellular calcium stimulates G-cell proliferation through the calcium sensing receptor, which can very well explain the G-cell hyperplasia, the relative low frequency of loss of heterozygosity in MEN1-associated gastrinomas, and MEN1-like phenotypes in MEN1 mutation negative individuals with parathyroid adenomas (13). The proposed parathyroid-gut axis implies that a proportion of the MEN1-syndrome associated gastrinomas is hyperplastic, which is consistent with the fact that parathyroid surgery can cure hypergastrinemia in patients with MEN1-syndrome (14). 


\section{PART 2. EPIGENETIC DIFFERENTIATION REFINES NEUROENDOCRINE TUMOR CLASSIFICATION}

\subsection{Biomarkers of behavior}

Behavior of pancreatic neuroendocrine tumors is notoriously heterogeneous, ranging from indolent to aggressive. Many biomarkers have been suggested to predict behavior and improve pre- and post-operative clinical decision making with the goal to reserve pancreatic surgery for those at risk of metastatic disease and accordingly personalize follow-up. Clinicopathological characteristics that are currently used to predict behavior include tumor size, WHO tumor grade by Ki-67 labeling or mitotic index, and presence of lymph node metastases $(15,16)$. Nevertheless, small WHO grade 1 tumors may still metastasize (17), and detecting lymph node metastases can be difficult pre-operatively. Therefore, additional biomarkers of behavior are a key unmet need.

Novel biomarkers, including ALT, ATRX/DAXX loss and the epigenetic subtype surrogate markers ARX/PDX1 have shown prognostic value in various retrospective cohorts of patients with pancreatic neuroendocrine tumors $(2,4,18-20)$. Nevertheless, it remained unknown if these markers can reliably be determined in the preoperative setting. In chapter 6, we determined ALT, ARX, PDX1 and tumor grade in 13 endoscopic ultrasound cytology specimens and matched surgical specimens as reference standard. Although this study was limited in size, we show that ALT, ARX, and PDX1 can reliably be determined pre-operatively (Kappa $>0.70$ ), while tumor grade is often underestimated (Kappa 0.28), which is consistent with the literature $(21,22)$.

In chapter 7, we determined the prognostic value of ALT, ARX, and PDX1 in the most common functional pancreatic tumor: insulinoma. Surgery is performed in all patients with insulinoma to treat severe hypoglycemic events. After surgery, most patients are cured from insulinoma, but some patients (5-10\%) develop metastases after surgery and have a poor prognosis. In our cohort of 37 sporadic insulinomas, five patients developed liver metastases. All non-metastatic primary insulinomas were small $(<3 \mathrm{~cm})$, lacked ALT and showed a uniform PDX1-positive/ARX-negative transcription factor expression pattern, typical for non-neoplastic pancreatic beta-cells. In contrast, all patients with metastatic insulinomas had primary tumors $>3 \mathrm{~cm}$, and four out of five had ALT. Furthermore, all five metastatic insulinomas expressed ARX (of which four were also PDX1-positive). These surprising findings made us consider the following hypothesis: 
Metastatic insulinomas are non-functional pancreatic neuroendocrine tumors with acquired insulin production.

In the first place, this hypothesis is supported by the fact that metastatic insulinomas are often detected when very large (mean size $6.16 \mathrm{~cm}$ [SD 2.63]), while indolent insulinomas are small (mean $1.48 \mathrm{~cm}$ [SD 0.45]). Insulinomas are usually detected by symptomatic hypoglycemia. Therefore, these metastatic insulinomas were per definition non-functional for a period of time until they grew to the size at which they became symptomatic. A recent review supports this hypothesis, and several case-reports of non-functional pancreatic neuroendocrine tumors with metachronous insulin production during follow-up have been reported $(23,24)$.

Second, ATRXIDAXX mutations are rarely seen in insulinomas, while relatively common in non-functional pancreatic neuroendocrine tumors. A recent whole genome sequencing study that stratified neuroendocrine tumors by copy number variations found that sporadic mutations in MEN1, ATRX or DAXX are absent in indolent insulinomas $(25,26)$. Instead, indolent insulinomas are regularly copy number neutral and $Y Y 1$-mutated (59\%), or characterized by copy number amplifications without recurrent mutations. In contrast, nonfunctional pancreatic neuroendocrine tumors with copy number amplifications/deletions were characterized by ATRX or DAXX mutations (53\%) while YY1 mutations were absent. The fact that 4 out of 5 metastatic insulinomas in our study had ALT, shows that they share their tumorigenic pathway with non-functional pancreatic neuroendocrine tumors, distinct from their indolent insulinoma counterpart.

Third, the aberrant ARX/PDX1 expression pattern of metastatic insulinomas compared to normal beta-cells and indolent insulinomas is remarkable, and suggests a different differentiation. ARX is expressed in pancreatic alpha-, epsilon- and gamma-cells, and is commonly expressed in non-functional pancreatic neuroendocrine tumors $(18,19,27$, 28). Furthermore, there is a strong correlation of ATRX/DAXX mutations and alpha-cell differentiation in non-functional pancreatic neuroendocrine tumors (18, 19). Therefore, the presence of ALT in metastatic insulinomas might further point to an alpha-like state of epigenetic differentiation, which could be the result of a non-beta-cell of origin. 
In chapter 8, the postoperative association of relapse free survival and ALT, ATRX/DAXX, ARX, PDX1, and clinicopathological markers is validated in an international, well-defined retrospective cohort of 561 non-functional pancreatic neuroendocrine tumors without synchronous distant metastases at surgery. Furthermore, in a sub-analysis, we determined the postoperative prognostic value for the clinically challenging tumors $\leq 2 \mathrm{~cm}$ without lymph node metastases, for which guidelines for surgery are ambiguous $(15,16)$. Unexpectedly, differential ARX or PDX1 expression did not show a univariate association with relapse after surgery. ARX-positive/PDX1-negative tumors did associate with relapse compared to PDX1-positive/ARX-negative tumors, however this significant association was lost when ALT or ATRX/DAXX was added in a multivariate model. Indeed, tumors with ARX expression had a significantly higher proportion of ALT, which can explain this confounding effect. In multivariate cox-regression analysis that included biomarkers significant in univariate analysis, only tumor size $>2 \mathrm{~cm}$, lymph node metastases, and ALT (or substituted by ATRX/ DAXX loss) independently predicted relapse after surgery. For the tumors $\leq 2 \mathrm{~cm}$ without lymph node metastases, a multivariate analysis showed that only ALT (or ATRX/DAXX) was independently associated with relapse after surgery, but not tumor size.

Previous studies that assessed these biomarkers were largely restricted to small, single center cohorts, which often also included functional or syndrome related neuroendocrine tumors (18-20). In addition, patients with synchronous metastases at surgery were included. The study presented in the thesis represents the first to specifically look at the prognostic value of these biomarkers in a patient group that is often encountered in the clinic: sporadic patients without synchronous metastases at surgery. At any point during follow up, the uncorrected risk of relapse is about 5.6 times higher in patients with ALT-positive tumors compared to patients with ALT-negative tumors, which was the most powerful independent predictor of relapse. Notably, an almost identical hazard ratio was found in the clinically challenging group of non-functional pancreatic neuroendocrine tumors $\leq 2.0$ without lymph node involvement. This study therefore presents compelling evidence to advocate for postoperative assessment of ALT or ATRX/DAXX status in all patients with non-functional neuroendocrine tumors without synchronous metastases at surgery. Furthermore, based on the convincing results in tumors smaller than $\leq 2.0 \mathrm{~cm}$, pre-operative determination of ALT or ATRX/DAXX needs to be considered for small tumors, although prospective studies must be performed to determine the added value above conventional indications for surgery. 


\subsection{Predicting origin by endocrine differentiation}

As secondary objective in chapter $\mathbf{8}$, we studied the presence and prognostic value of the biomarkers ARX, PDX1, ALT, and ATRX/DAXX in 107 pancreatic neuroendocrine tumor metastases and 654 non-pancreatic neuroendocrine tumors. Interestingly, ARX or PDX1 expression was absent in all pulmonary, jejunal, and ileal neuroendocrine tumors, while positive in a proportion of pancreatic, gastric, duodenal, appendiceal and rectal neuroendocrine tumors. For PDX1, these results are consistent with previous studies (2933), while the use of ARX immunohistochemistry was not reported before in this context. Furthermore, ATRX or DAXX loss and ALT were highly specific for pancreatic neuroendocrine tumors, confirming a previous study (34). As expected, few pulmonary NETs had ATRX loss (35). In conclusion, although no prognostic value for these markers was found for nonpancreatic neuroendocrine tumors, they might have a future role in predicting the site origin of neuroendocrine tumors of unknown primary.

As the proportions of ARX or PDX1 expressing tumors were roughly similar in the cohort of 561 primary pancreatic neuroendocrine tumors and 107 pancreatic neuroendocrine metastases, we considered ARX/PDX1 transcription factor expression might be clonally preserved in primary tumors and their subsequent metastases. In chapter 9 , we proved this point by describing eighteen patients with unifocal sporadic pancreatic neuroendocrine tumors (both functional and non-functional), in which most (96\%) of 50 metastases showed a transcription factor profile similar to the primary tumor. We then used this principle to get insight in the metastatic patterns in ten patients with MEN1-syndrome. MEN1 patients often have numerous primary duodenopancreatic neuroendocrine tumors, which makes it challenging to determine the origin of metastases. We found that in six MEN1 patients with suspected gastrinoma (i.e., hypergastrinemia) - without exception - all lymph node metastases found in duodenopancreatectomy specimens clustered with duodenal gastrinomas (suggesting relatedness) and not pancreatic neuroendocrine tumors. This is important, as these duodenal tumors can be microscopically small $(<5 \mathrm{~mm})$, and are regularly missed on conventional or functional imaging, while the generally larger pancreatic neuroendocrine tumors are often detected. Therefore, lymph node metastases near the pancreatic head should be attributed to pancreatic neuroendocrine tumors with caution, especially in the setting of hypergastrinemia. Moreover, this underlines the necessity of performing duodenopancreatectomies to cure patients of metastatic gastrinoma in MEN1 syndrome. 
In the last chapter (chapter 10), we aimed to improve the diagnostic workup for a longstanding clinical challenge: patients that present with neuroendocrine tumors of unknown primaries. Although several immunohistochemical markers or panels have been proposed to determine origin (including PDX1, as described in in chapter 8), they are often limited by a lack of sensitivity, resulting in overall accuracy $<80 \%$. As mentioned in the introduction of this thesis, whole genome methylation is one of the epigenetic marks that can be used to deduce epigenetic tumor morphology, which is highly cell- and origin-specific. As proof of concept, we compiled a training cohort of whole genome methylation data of pancreatic, pulmonary, and small intestinal neuroendocrine tumors, as they are the most common origin of distant metastases (18, 36-38). Informative epigenomic regions were selected by ranking $\mathrm{CPG}$ sites via the mean absolute standard deviation during repeated downsampling by origin class. A random forest model was combined with logistic ridge regression. During cross-validation and after applying the model to a test cohort of 165 primary neuroendocrine tumors and 28 metastases of known origin, accuracy remained $>95 \%$, a significant improvement compared to the current immunohistochemical panels. Furthermore, we show that the predictive model (named NEN-ID) is robust against low tumor-purity of low-quality data. We illustrate the use NEN-ID by successfully applying it to three neuroendocrine tumors of unknown primary, and present the model as a free to use online application. 


\section{PANCREATIC NEUROENDOCRINE TUMORIGENESIS: NEW INSIGHTS BY EPIGENETIC NEUROENDOCRINE TUMOR MORPHOLOGY}

Despite the fact that we could not confirm the independent prognostic value of ARX/PDX1 subtypes in sporadic non-functional pancreatic neuroendocrine tumors, the recent reports on endocrine differentiation in non-functional pancreatic neuroendocrine tumors have caused a paradigm shift in our understanding of pancreatic neuroendocrine tumorigenesis $(18,19)$. Interestingly, we did confirm the strong association of the alpha differentiation (ARX expression) with the presence of ATRXIDAXX inactivation or ALT in chapter 8. This intriguing finding, in which ATRXIDAXX or MEN1 mutant neuroendocrine tumors often seem to show epigenetic alpha-cell characteristics has now been replicated by many others $(18,19,39-42)$. This shows how epigenetic tumor morphology can uncover subtle differentiation classes that associate with specific tumorigenic mechanisms and behavior, and can improve the traditional histopathological tumor classification described in the first part of this thesis. However, it seems by the most recent studies on the subject, that the use ARX and PDX1 as substitute markers of these different classes might have been an oversimplification of a complex, multi-staged tumorigenic process.

Di Domenico et al. recently performed an elegant phylo-epigenetic analysis of 125 functional and non-functional neuroendocrine tumors, using normal alpha- and betacells as root nodes (40). Interestingly, a group of mostly non-functional neuroendocrine tumors was identified with a high degree of alpha-cell similarity, characterized by MEN1 mutations, limited copy number events, and the lack of ATRX and DAXX mutations, likely corresponding with the copy-neutral type of non-functional neuroendocrine tumors described by the major whole genome sequencing study with 211 neuroendocrine tumors by Hong et al (25). In both studies, this subgroup had a very favorable prognosis $(25,40)$. Another subgroup showed a high similarity to beta-cells, and lacked MEN1, ATRXIDAXX mutations or recurrent chromosomal losses, and almost entirely consisted of sporadic insulinomas (40). As sporadic insulinomas have a distinct pathway of tumorigenesis, and clinically have a different presentation and treatment, comparing prognosis with nonfunctional pancreatic neuroendocrine tumors is not really informative (25). Nevertheless, the beta-like neuroendocrine tumors had excellent prognosis. Last, there was a larger group in between, with a reduced but still significant alpha-cell similarity, a weak beta-cell similarity, recurrent MEN1, ATRX and DAXX mutations, and frequent chromosomal losses 
and gains. Similar to the subtype with recurrent amplifications or deletions as described by Hong et al., this dedifferentiated or "intermediate" subgroup showed a significantly worse prognosis compared to the well-differentiated alpha-like neuroendocrine tumors $(25$, 40). Importantly, ARX expression was present in all alpha-like and most intermediate like tumors, but was absent in almost all insulinomas (40), except for two cases with metastases, which confirms the association found in chapter 7 of this thesis. These results suggest that ARX immunohistochemistry poorly reflects the state of malignant progression, but rather hints at the progressive tumorigenic (MEN1, + ATRX and DAXX mutant) pathway travelled (figure 1, table 1).

The proposed alpha-dedifferentiation pathway of sporadic tumorigenesis, which often seems to be initiated by MEN1 mutations, calls into question how MEN1-syndrome associated pancreatic non-functional "beta-like" tumors can exist, as was described in the original report (19) and in chapter 9: around one out of five microadenomas and one in three neuroendocrine tumors in patients is PDX1-positive/ARX-negative. Moreover, while insulinomas are relatively common in MEN1-syndrome (43), MEN1 mutations are never seen in sporadic insulinomas $(25,26)$, which challenges the assumption that MEN1 inactivation alone can induce functional beta-like tumors (i.e. insulinomas). Indeed, in chapter 9, we show that non-functional pancreatic beta-like neuroendocrine tumors are more frequent than functional beta-like tumors in patients with MEN1-syndrome. In addition, in a murine MEN1-syndrome model, beta-cell specific Men1-ablation results in delayed tumor

Table 1. PanNET classification based on genetic and epigenetic signatures.

\begin{tabular}{|c|c|c|c|c|c|c|}
\hline & $\begin{array}{l}\text { NF-PanNET } \\
\text { subtype } 1\end{array}$ & $\begin{array}{l}\text { NF-PanNET } \\
\text { subtype } 2\end{array}$ & $\begin{array}{l}\text { NF-PanNET } 3 \\
\text { subtype }\end{array}$ & $\begin{array}{l}\text { Insulinoma } \\
\text { subtype } 1\end{array}$ & $\begin{array}{l}\text { Insulinoma } \\
\text { subtype } 2\end{array}$ & $\begin{array}{l}\text { Insulinoma } \\
\text { subtype } 3^{*}\end{array}$ \\
\hline $\begin{array}{l}\text { Epigenetic } \\
\text { Similarities }\end{array}$ & Alpha-like & $\begin{array}{l}\text { Alpha-like> } \\
\text { Beta-like }\end{array}$ & Beta-like/ other & Beta-like & Beta-like & $\begin{array}{l}\text { Alpha-like> } \\
\text { Beta-like }\end{array}$ \\
\hline $\begin{array}{l}\text { Epigenetic } \\
\text { Differentiation }\end{array}$ & $\begin{array}{l}\text { Well- } \\
\text { differentiated }\end{array}$ & $\begin{array}{l}\text { Intermediate } \\
\text { differentiation }\end{array}$ & unknown & $\begin{array}{l}\text { Well- } \\
\text { differentiated }\end{array}$ & $\begin{array}{l}\text { Well- } \\
\text { differentiated }\end{array}$ & $\begin{array}{l}\text { De- } \\
\text { differentiated? }\end{array}$ \\
\hline MEN1 & ++ & +++ & $+/-$ & - & - & $?$ \\
\hline$A T R X / D A X X$ & + & +++ & - & - & - & ++ \\
\hline YY1 & - & - & - & +++ & - & $?$ \\
\hline mTOR pathway & + & +++ & + & - & - & $?$ \\
\hline $\begin{array}{l}\text { Copy number } \\
\text { profiles }\end{array}$ & $\begin{array}{l}\text { Copy number } \\
\text { neutral }\end{array}$ & $\begin{array}{l}\text { Copy number } \\
\text { amplifications / } \\
\text { deletions }\end{array}$ & $\begin{array}{l}\text { Copy number } \\
\text { neutral }\end{array}$ & $\begin{array}{l}\text { Copy number } \\
\text { neutral }\end{array}$ & $\begin{array}{l}\text { Recurrent } \\
\text { amplifications }(7, \\
3 p, 5 q \text { and } 13 q,)^{\prime}\end{array}$ & $?$ \\
\hline $\begin{array}{l}\text { Predominant } \\
\text { Grade }\end{array}$ & G1 & $\mathrm{G} 2$ & G1 & G1 & G1 & $\mathrm{G} 2$ \\
\hline Mean size* & $3 \mathrm{~cm}$ & $4 \mathrm{~cm}$ & $3 \mathrm{~cm}$ & $<2 \mathrm{~cm}$ & $<2 \mathrm{~cm}$ & $>3 \mathrm{~cm}$ \\
\hline Prognosis & Favorable & Poor & Favorable & Favorable & Favorable & Poor \\
\hline
\end{tabular}

Summary of recent insights in surgically resected sporadic pancreatic neuroendocrine tumor subtypes, main subgroups based on Hong et al. and other studies are matched based on clinical characteristics, copy number profiling recurrent mutations, and differentiation.

* possibly the same as NF-PanNET-2 wit acquired symptomatic insulin production.

Abbreviations: NF-PanNET non-functional pancreatic neuroendocrine tumor, G grade. 


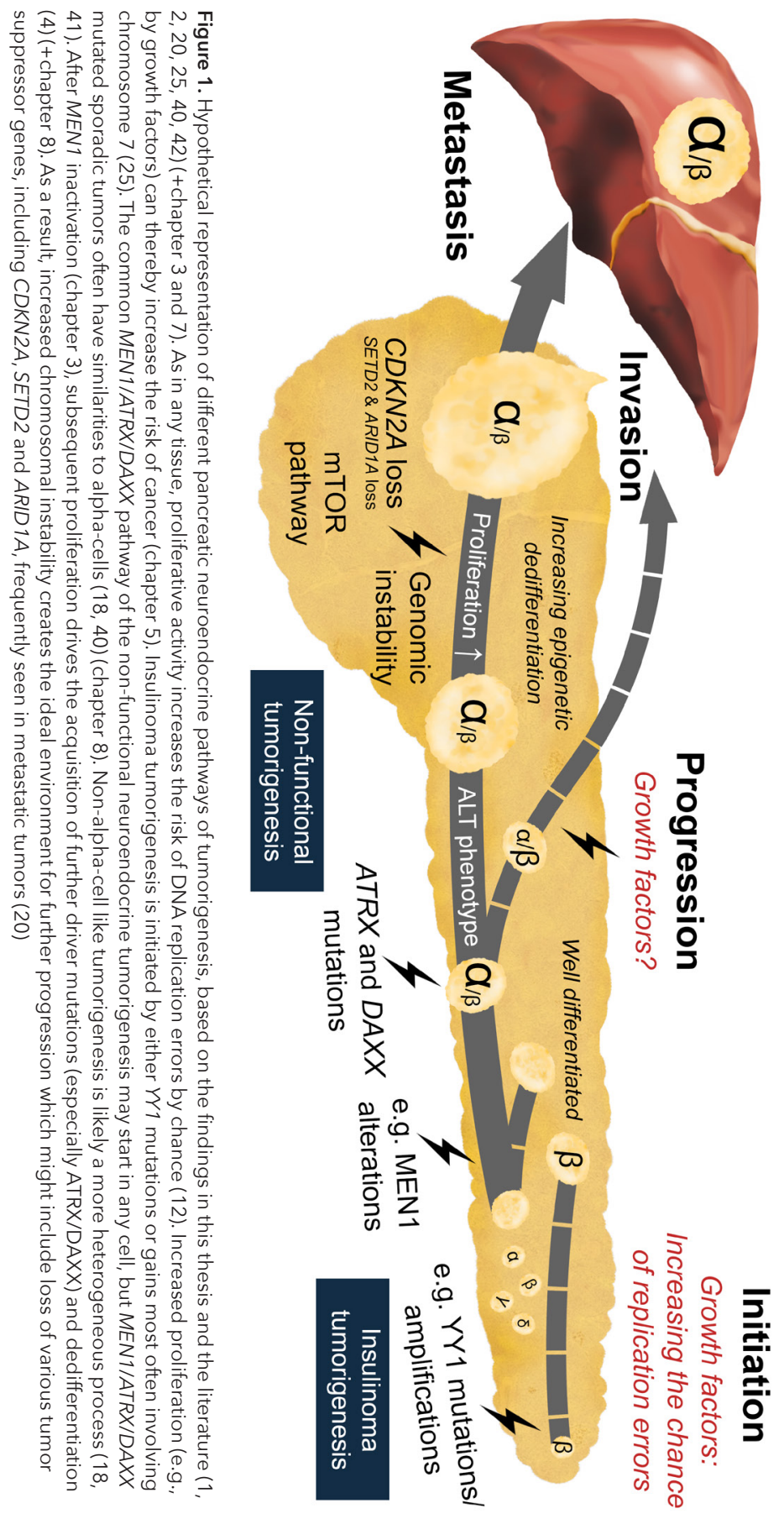


appearance, suggesting that additional events are needed for insulinoma tumorigenesis (44). It therefore seems that MEN1 inactivation alone cannot initiate insulinoma formation.

Few possible explanations can be found for these discrepant findings. In the first place, the studies describing beta-like tumors with MEN1 mutations used immunohistochemical ARX/PDX1 expression as surrogate marker of endocrine differentiation (19) (chapter 8,9), while others used epigenomic/transcriptomic approaches $(18,40,41)$. Alpha- or beta-cell similarity cannot be determined by immunohistochemical ARX/PDX1 alone, and various other alpha/beta-cell markers (e.g., IRX2/MAFB(27)) and global epigenetic similarities were not determined. Thus, it cannot be ruled out that ARX-negative/PDX1-positive tumors or insulinomas in MEN1-syndrome have underlying alpha-cell similarities.

A second option, although speculative, builds on the fact that most sporadic MEN1-mutated pancreatic neuroendocrine tumors are dedifferentiated compared to alpha- and betacells (40), and can therefore be considered "immature". In 1957, Waddington described a figurative epigenetic landscape to explain cell fate, in which immature-cells [marbles] roll down a sloping surface with bifurcating grooves leading into divisive valleys of mature differentiation (Figure 2) (45). Within competent tissue, the presence or absence of gene expression or environmental stimuli mold the ridges and hills of this channeling landscape to favor certain pathways. Within endocrine cells (a competent tissue), MEN1 is a known epigenetic landscaper through its interaction with histone modifying proteins MLL1 and MLL2 (46). Therefore, loss of MEN1 might fundamentally change the playing field, resulting in unstable state of differentiation, increasing the vulnerability for dedifferentiation or transdifferentiation (Figure 2).

MEN1-syndrome is a multifocal and multihormonal endocrine tumor disease, and endocrine hormones or their downstream effects can influence other endocrine tissues (chapter 4, 5). For example, prolactin has shown to promote both alpha- and beta-cell growth in pregnancy (47-49). Prolactinomas are common in patients of MEN1-syndrome. Moreover, diabetes research has shown that glucagon like peptide 1 (GLP1) can promote alpha-to-beta transdifferentiation, by increasing several beta-cell markers (including PDX1) in alpha-cells $(50,51)$. Pancreatic neuroendocrine tumors commonly secrete GLP1, and in combination with glucagon can even cause beta-cell hyperplasia $(52,53)$. The physiological cross-talk observed between endocrine cells raises the possibility of neuroendocrine tumor cross-talk in patients with multiple endocrine tumors (e.g., in MEN1-syndrome), which may 


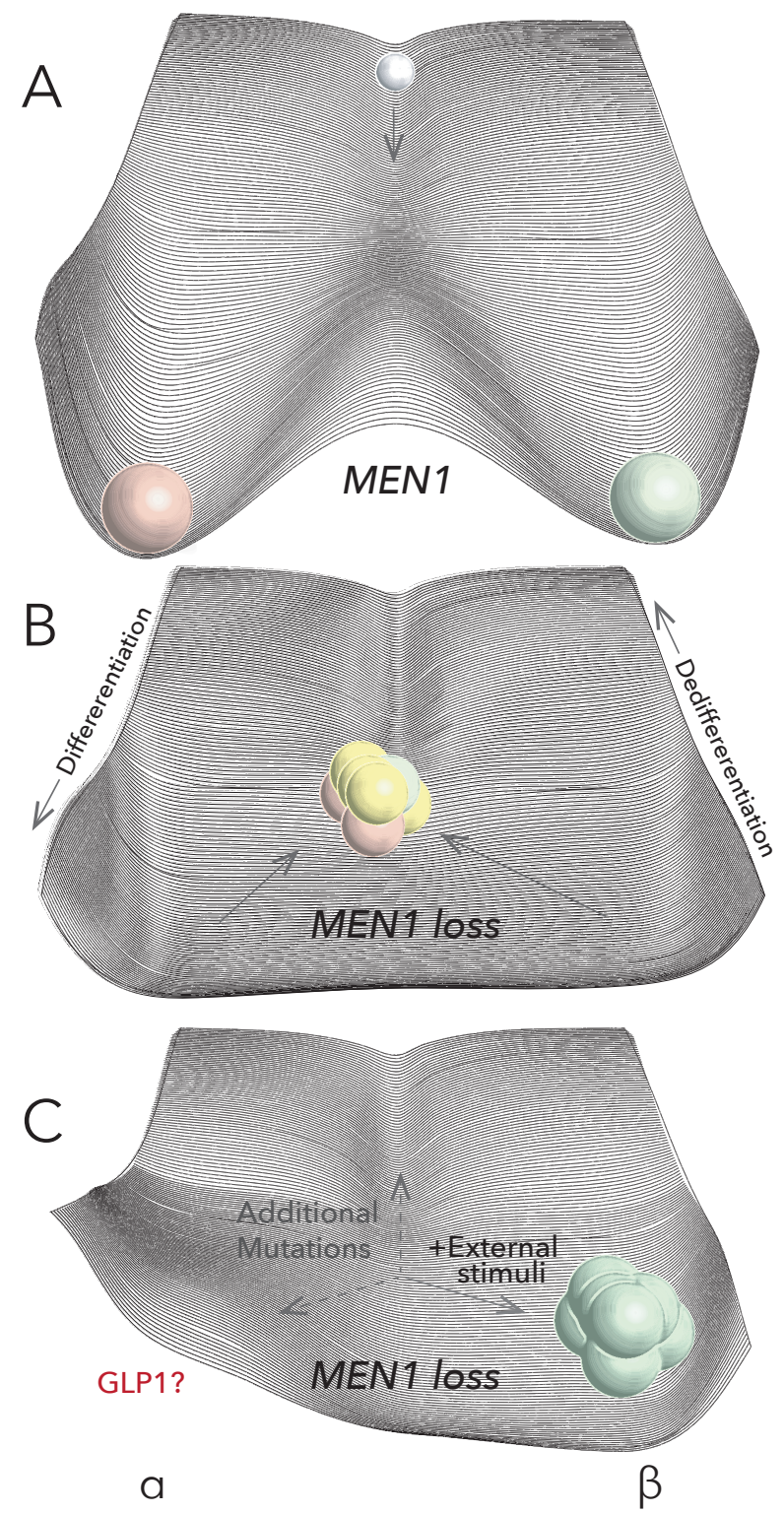

Figure 2. Impression of epigenetic neuroendocrine tumor landscape (morphology) change after MEN1 inactivation. A, immature cells develop into differentiated endocrine cells with retained MEN1. B, MEN1 loss initiates tumorigenesis, but also relevels the epigenetic landscape, making cells vulnerable for changes in differentiation, including dedifferentiation (example shown). C, external stimuli may be present particularly in patients with multiple endocrine neoplasia (e.g., GLP1), which may push endocrine cells of unstable (de)differentiation into compensatory (trans)differentiation depending on the stimulus. Author's interpretation of Waddington's Epigenetic Landscapes (45). 
play a role in the pathways of differentiation or transdifferentiation.

In this regard, two murine MEN1-syndrome models specifically studied the effect of alphacell or beta-cell specific Men1-ablation. Interestingly, this leads to the development of both glucagonomas, mixed glucagon/insulin expressing neuroendocrine tumors and insulinomas $(54,55)$. Alpha-cell specific Men1 inactivation over time leads to increased numbers of Men1-deficient cells that show an "intermediate" subtype, with both alphaand beta-like characteristics, and eventually an increased number of Men1-deficient insulin expressing cells (54). Beta-cell specific Men1 inactivation over time resulted in a gradual decrease in expression of mature beta-cell transcription factors in beta-cells (55). It seems that endocrine cells try to balance their presence with other endocrine cells. For example, extreme beta-cell loss in mice results in compensatory alpha-to-beta-cell transdifferentiation (56). The fact that this even happens in alpha- and beta-specific MEN1 models in mice (which supposedly do not have other endocrine tumors), suggests that intrapancreatic neuroendocrine crosstalk is enough to initiate neuroendocrine transdifferentiation. Although highly speculative, it is possible that in reaction to each other's secretions (e.g., GLP1), pancreatic neuroendocrine tumors or cells are forced to choose a side, a state of differentiation that could later become permanent by genetic assimilation. This hypothesis could explain the observation that many sporadic non-functional MEN1 mutant tumors remain in the "intermediate/dedifferentiated" category with some alpha-cell characteristics (40), as they do not sense any hormonal imbalance. In MEN1-syndrome, these immature tumors could be forced into a compensatory state of differentiation by secretions of other neuroendocrine tumors. Nevertheless, future research is necessary to explain why beta-like neuroendocrine tumors are observed in MEN1-syndrome.

\section{Future clinical and research perspectives}

This thesis confirms the strong prognostic value of ALT as biomarker of behavior in small and large sporadic non-functional pancreatic neuroendocrine tumors and insulinomas. We therefore believe that ATRX/DAXX immunohistochemistry and/or telomere fluorescence in situ hybridization should be implemented as prognostic tool in the post-operative work-up of all patients with neuroendocrine tumors. In addition, as ATRX/DAXX or ALT can reliably be determined pre-operatively, there is an urgent need for prospective studies to evaluate its benefit in selecting patients with small $(<2 \mathrm{~cm})$ pancreatic neuroendocrine tumors for surgery. 
Our increased appreciation of the role of epigenetics in differentiation and cell-type specific tumorigenic vulnerabilities has caused a paradigm shift in the neuroendocrine tumor research field. It has opened our eyes for the vast genetic and epigenetic diversity within tumors that were previously considered to be a single entity. It is possible, although not by simple ARX and PDX1 immunohistochemistry, that alpha- and beta-like subtypes will be used for prognostic classification in the future. Furthermore, differentiation specific pathways might reveal novel targets for treatment. The initiation of alpha-cell tumorigenesis in GCHN has increased our awareness that neuroendocrine cells are highly responsive to stimuli and growth factors. This resulted in several additional research questions: Could a calcium dependency be exploited to reduce gastrinoma tumorigenesis in MEN1syndrome? Could the higher risk of pancreatic neuroendocrine tumors in patients with obesity and diabetes - one of the few established risk factors - be the result of fatty liver disease/diabetes associated hyperaminoacidemia?

One of the reasons we now appreciate the subtle differences in neuroendocrine differentiation, is the availability of relatively cheap, reproducible arrays to determine the epigenetic tumor morphology. Nevertheless, because of the rarity of neuroendocrine tumors, many neuroendocrine tumor types remain poorly studied. One of the most important objectives in the near future is not only to extend the NEN-ID origin classifier to include all different neuroendocrine neoplasm origins, but also to predict prognosis and treatment response. While neuroendocrine tumors will always remain dazzling, we may then be able to classify with our eyes closed. 


\section{REFERENCES}

1. Scarpa A, Chang DK, Nones K, Corbo V, Patch A-M, Bailey $\mathrm{P}$, et al. Whole-genome landscape of pancreatic neuroendocrine tumours. Nature. 2017;543(7643):65-71.

2. Heaphy $\mathrm{CM}$, de Wilde RF, Jiao Y, Klein AP, Edil BH, Shi $C$, et al. Altered telomeres in tumors with ATRX and DAXX mutations. Science. 2011;333(6041):425.

3. Ro C, Chai W, Yu VE, Yu R. Pancreatic neuroendocrine tumors: biology, diagnosis, and treatment. Chin J Cancer. 2013;32(6):312-24.

4. Kim JY, Brosnan-Cashman JA, An S, Kim SJ, Song KB, Kim MS, et al. Alternative Lengthening of Telomeres in Primary Pancreatic Neuroendocrine Tumors Is Associated with Aggressive Clinical Behavior and Poor Survival. Clin Cancer Res. 2017;23(6):1598-606.

5. Marinoni I, Kurrer AS, Vassella E, Dettmer M, Rudolph $T$, Banz V, et al. Loss of DAXX and ATRX are associated with chromosome instability and reduced survival of patients with pancreatic neuroendocrine tumors. Gastroenterology. 2014;146(2):453-60 e5.

6. Masliah-Planchon J, Levy D, Heron D, Giuliano F, Badens C, Freneaux P, et al. Does ATRX germline variation predispose to osteosarcoma? Three additional cases of osteosarcoma in two ATR-X syndrome patients. Eur J Hum Genet. 2018;26(8):1217. 21.

7. Debelenko LV, Zhuang Z, Emmert-Buck MR, Chandrasekharappa SC, Manickam P, Guru SC, et al. Allelic deletions on chromosome 11q13 in multiple endocrine neoplasia type 1 -associated and sporadic gastrinomas and pancreatic endocrine tumors. Cancer Res. 1997;57(11):2238-43.

8. Lubensky IA, Debelenko LV, Zhuang Z, Emmert-Buck MR, Dong Q, Chandrasekharappa S, et al. Allelic deletions on chromosome $11 \mathrm{q} 13$ in multiple tumors from individual MEN1 patients. Cancer Res. 1996;56(22):5272-8.

9. Perren A, Anlauf M, Henopp T, Rudolph T, Schmitt A, Raffel A, et al. Multiple endocrine neoplasia type 1 (MEN1): loss of one MEN1 allele in tumors and monohormonal endocrine cell clusters but not in islet hyperplasia of the pancreas. J Clin Endocri nol Metab. 2007;92(3):1118-28.

10. de Wilde RF, Heaphy CM, Maitra A, Meeker AK, Edil $\mathrm{BH}$, Wolfgang $\mathrm{CL}$, et al. Loss of ATRX or DAXX expression and concomitant acquisition of the alternative lengthening of telomeres phenotype are late events in a small subset of MEN-1 syndrome pancreatic neuroendocrine tumors. Mod Pathol. 2012;25(7):10339.

11. Sipos B, Sperveslage J, Anlauf M, Hoffmeister M, Henopp T, Buch S, et al. Glucagon cell hyperplasia and neoplasia with and without glucagon receptor mutations. J Clin Endocrinol Metab. 2015;100(5):E783-8.

12. Tomasetti C, Vogelstein B. Cancer etiology. Variation in cancer risk among tissues can be explained by the number of stem cell divisions. Science. 2015;347(6217):78-81.

13. Kovesdi A, Toth M, Butz H, Szucs N, Sarman B, Pusztai $P$, et al. True MEN1 or phenocopy? Evidence for geno-phenotypic correlations in MEN1 syndrome. Endocrine. 2019;65(2):451-9

14. Norton JA, Venzon DJ, Berna MJ, Alexander HR, Fraker DL, Libutti SK, et al. Prospective study of surgery for primary hyperparathyroidism (HPT) in multiple endocrine neoplasia-type 1 and Zollinger-Ellison syndrome: long-term outcome of a more virulent form of HPT. Ann Surg. 2008;247(3):501-10. 15. Falconi M, Eriksson B, Kaltsas G, Bartsch DK, Capdevila J, Caplin M, et al. ENETS Consensus Guidelines Update for the Management of Patients with Functional Pancreatic Neuroendocrine Tumors and Non-Functional Pancreatic Neuroendocrine Tumors. Neuroendocrinology. 2016;103(2):153-71. 16. Howe JR, Merchant NB, Conrad C, Keutgen XM, Hallet J, Drebin JA, et al. The North American Neuroendocrine Tumor Society Consensus Paper on the Surgical Management of Pancreatic Neuroendocrine Tumors. Pancreas. 2020;49(1):1-33. 17. Pea A, Yu J, Marchionni L, Noe M, Luchini C, Pulvirenti A, et al. Genetic Analysis of Small Well-differentiated Pancreatic Neuroendocrine Tumors Identifies Subgroups With Differing Risks of Liver Metastases. Ann Surg. 2020;271(3):566-73.

18. Chan CS, Laddha SV, Lewis PW, Koletsky MS, Robzyk K, Da Silva E, et al. ATRX, DAXX or MEN1 mutant pancreatic neuroendocrine tumors are a distinct alpha-cell signature subgroup. Nat Commun. 2018;9(1):4158.

19. Cejas P, Drier Y, Dreijerink KMA, Brosens LAA, Deshpande $V$, Epstein $C B$, et al. Enhancer signatures stratify and predict outcomes of non-functional pancreatic neuroendocrine tumors. Nat Med. 2019;25(8):1260-5.

20. Roy S, LaFramboise WA, Liu T-C, Cao D, Luvison A, Miller $C$, et al. Loss of Chromatin-Remodeling Proteins and/or CDKN2A Associates With Metastasis of Pancreatic Neuroendocrine Tumors and Reduced Patient Survival Times. Gastroenterology. 2018;154(8):2060-3.e8.

21. Boutsen L, Jouret-Mourin A, Borbath I, van Maanen A, Weynand B. Accuracy of Pancreatic Neuroendocrine Tumour Grading by Endoscopic Ultrasound-Guided Fine Needle Aspiration: Analysis of a Large Cohort and Perspectives for Improvement. Neuroendocrinology. 2018;106(2):158-66.

22. VandenBussche CJ, Allison DB, Graham MK, Charu $\mathrm{V}$, Lennon AM, Wolfgang $C L$, et al. Alternative lengthening of telomeres and ATRX/DAXX loss can be reliably detected in FNAs of pancreatic neuroendocrine tumors. Cancer Cytopathol. 2017; 125(7):544-51.

23. Yu R. Malignant Insulinoma Is Largely Derived From Nonfunctioning Pancreatic Neuroendocrine Tumors: A Contemporary View. Pancreas. 2020;49(6):733-6.

24. Arslan MS, Ozbek M, Karakose M, Tutal E, Ucan B, Yilmazer D, et al. Transformation of nonfunctioning pancreatic tumor into malignant insulinoma after 3 years: an uncommon clinical course of insulinoma. Arch Endocrinol Metab. 2015;59(3):270-2. 25. Hong X, Qiao S, Li F, Wang W, Jiang R, Wu H, et al. Whole-genome sequencing reveals distinct genetic bases for insulinomas and non-functional pancreatic neuroendocrine tumours: leading to a new classification system. Gut. 2020;69(5):877-87. 26. Wang $\mathrm{H}$, Bender A, Wang P, Karakose E, Inabnet WB, Libutti SK, et al. Insights into beta cell regeneration for diabetes via integration of molecular landscapes in human insulinomas. Nat Commun. 2017;8(1):767.

27. Muraro MJ, Dharmadhikari G, Grun D, Groen N Dielen $T$, Jansen $E$, et al. A Single-Cell Transcriptome Atlas of the 
Human Pancreas. Cell Syst. 2016;3(4):385-94 e3.

28. Baron M, Veres A, Wolock SL, Faust AL, Gaujoux R, Vetere A, et al. A Single-Cell Transcriptomic Map of the Human and Mouse Pancreas Reveals Inter- and Intra-cell Population Structure. Cell Syst. 2016;3(4):346-60 e4

29. Srivastava A, Hornick JL. Immunohistochemical staining for CDX-2, PDX-1, NESP-55, and TTF-1 can help distinguish gastrointestinal carcinoid tumors from pancreatic endocrine and pulmonary carcinoid tumors. Am J Surg Pathol. 2009;33(4):626-32. 30. Hermann G, Konukiewitz B, Schmitt A, Perren A, Kloppel G. Hormonally defined pancreatic and duodenal neuroendocrine tumors differ in their transcription factor signatures: expression of ISL1, PDX1, NGN3, and CDX2. Virchows Arch. 2011;459(2):14754.

31. Yang Z, Klimstra DS, Hruban RH, Tang LH. Immunohistochemical Characterization of the Origins of Metastatic Well-differentiated Neuroendocrine Tumors to the Liver. Am J Surg Pathol. 2017;41(7):915-22.

32. Yang MX, Coates RF, Ambaye A, Cortright V, Mitchell JM, Buskey AM, et al. NKX2.2, PDX-1 and CDX-2 as potential biomarkers to differentiate well-differentiated neuroendocrine tumors. Biomark Res. 2018;6:15.

33. Gurevich LE, Proshchina AE, Voronkova IA, Ashevs kaya VE, Korosteleva PA, Dolzhansky OV. [Differential diagnostic value of the expression of the transcription factor PDX-1 in neuroendocrine and non-neuroendocrine tumors of the pancreas and other organs]. Arkh Patol. 2019;81(5):11-21.

34. Dogeas E, Karagkounis G, Heaphy CM, Hirose K, Pawlik TM, Wolfgang $C L$, et al. Alternative lengthening of telomeres predicts site of origin in neuroendocrine tumor liver metastases. $J$ Am Coll Surg. 2014;218(4):628-35.

35. Terra Md S, Xie Md Ph DH, Boland Md JM, Mansfield Md AS, Molina Md Ph DJ, Roden Md AC. Loss of ATRX expression predicts worse prognosis in pulmonary carcinoid tumors. Hum Pathol. 2019;94:78-85.

36. Karpathakis A, Dibra H, Pipinikas C, Feber A, Morris T, Francis J, et al. Prognostic Impact of Novel Molecular Subtypes of Small Intestinal Neuroendocrine Tumor. Clin Cancer Res. 2016;22(1):250-8.

37. Laddha SV, da Silva EM, Robzyk K, Untch BR, Ke H, Rekhtman N, et al. Integrative Genomic Characterization Identifies Molecular Subtypes of Lung Carcinoids. Cancer Res. 2019;79(17):4339-47.

38. Yao JC, Hassan M, Phan A, Dagohoy C, Leary C, Mares $J E$, et al. One hundred years after "carcinoid": epidemiology of and prognostic factors for neuroendocrine tumors in 35,825 cases in the United States. J Clin Oncol. 2008;26(18):3063-72.

39. Boons G, Vandamme T, Ibrahim J, Roeyen G, Driessen A, Peeters D, et al. PDX1 DNA Methylation Distinguishes Two Subtypes of Pancreatic Neuroendocrine Neoplasms with a Different Prognosis. Cancers (Basel). 2020;12(6).

40. Di Domenico A, Pipinikas CP, Maire RS, Brautigam K, Simillion C, Dettmer MS, et al. Epigenetic landscape of pancreatic neuroendocrine tumours reveals distinct cells of origin and means of tumour progression. Commun Biol. 2020;3(1):740.

41. Simon T, Mamlouk S, Riemer P, Bormann F, Klinger B, Menne A, et al. An Integrative Genetic, Epigenetic and Proteomic Characterization of Pancreatic Neuroendocrine Neoplasms
(PanNENs) defines Distinct Molecular Features of a- and $\beta$-cell like Subgroups. bioRxiv. 2020:2020.06.12.146811.

42. Lakis V, Lawlor RT, Newell F, Patch AM, Mafficini A, Sadanandam A, et al. DNA methylation patterns identify subgroups of pancreatic neuroendocrine tumors with clinical association. Commun Biol. 2021;4(1):155.

43. van Beek DJ, Nell S, Verkooijen HM, Borel Rinkes IHM, Valk GD, Vriens MR, et al. Surgery for multiple endocrine neoplasia type 1-related insulinoma: long-term outcomes in a large international cohort. Br J Surg. 2020;107(11):1489-99.

44. Crabtree JS, Scacheri PC, Ward JM, McNally SR, Swain GP, Montagna C, et al. Of mice and MEN1: Insulinomas in a conditional mouse knockout. Mol Cell Biol. 2003;23(17):6075-85.

45. Waddington C. The Strategy of the Genes. Allen.

Unwin, London; 1957.

46. Dreijerink KMA, Timmers HTM, Brown M. Twenty years of menin: emerging opportunities for restoration of transcriptional regulation in MEN1. Endocr Relat Cancer. 2017;24(10):T135-T45.

47. Karnik SK, Chen H, McLean GW, Heit JJ, Gu X, Zhang

$A Y$, et al. Menin controls growth of pancreatic beta-cells in pregnant mice and promotes gestational diabetes mellitus. Science. 2007;318(5851):806-9.

48. Szlapinski SK, Bennett J, Strutt BJ, Hill DJ. Increased

alpha and beta cell mass during mouse pregnancy is not dependent on transdifferentiation. Exp Biol Med (Maywood). 2020:1535370220972686.

49. Quesada-Candela C, Tuduri E, Marroqui L, Alonso-Magdalena P, Quesada I, Nadal A. Morphological and functional adaptations of pancreatic alpha-cells during late pregnancy in the mouse. Metabolism. 2020;102:153963.

50. Lee YS, Lee C, Choung JS, Jung HS, Jun HS.

Glucagon-Like Peptide 1 Increases beta-Cell Regeneration by Promoting alpha- to beta-Cell Transdifferentiation. Diabetes. 2018;67(12):2601-14

51. Zhang Z, Hu Y, Xu N, Zhou W, Yang L, Chen R, et al. A New Way for Beta Cell Neogenesis: Transdifferentiation from Alpha Cells Induced by Glucagon-Like Peptide 1. J Diabetes Res. 2019;2019:2583047.

52. Kim JY, Kim MS, Kim KS, Song KB, Lee SH, Hwang DW, et al. Clinicopathologic and prognostic significance of multiple hormone expression in pancreatic neuroendocrine tumors. Am J Surg Pathol. 2015;39(5):592-601.

53. Roberts RE, Zhao M, Whitelaw BC, Ramage J, Diaz-Cano S, le Roux CW, et al. GLP-1 and glucagon secretion from a pancreatic neuroendocrine tumor causing diabetes and hyperinsulinemic hypoglycemia. J Clin Endocrinol Metab. 2012;97(9):3039-45. 54. Lu J, Herrera PL, Carreira C, Bonnavion R, Seigne C, Calender A, et al. Alpha cell-specific Men1 ablation triggers the transdifferentiation of glucagon-expressing cells and insulinoma development. Gastroenterology. 2010;138(5):1954-65.

55. Li F, Su Y, Cheng Y, Jiang X, Peng Y, Li Y, et al. Conditional deletion of Men 1 in the pancreatic beta-cell leads to glucagon-expressing tumor development. Endocrinology. 2015;156(1):48-57.

56. Thorel F, Nepote V, Avril I, Kohno K, Desgraz R, Chera

$\mathrm{S}$, et al. Conversion of adult pancreatic alpha-cells to beta-cells

after extreme beta-cell loss. Nature. 2010;464(7292):1149-54. 


\section{Appendices}


Abbreviations

Dutch summary/Nederlandse samenvatting

Contributing authors

Curriculum vitae

List of publications

Acknowledgements 


\section{ABBREVIATIONS}

A

ACC Acinar Cell Carcinoma

ALT Alternative Lengthening of Telomeres

ARID1A AT-Rich Interaction Domain 1A

ARX Aristaless-Related Homeobox Gene

ATRX Alpha-Thalassemia/Mental Retardation Syndrome, X-Linked

AUC Area Under the Curve

B

BMIO Beta-Mixture Quantile

C

CaSR Calcium Sensing Receptor

CDKN1B Cyclin dependent Kinase Inhibitor 1B

CDKN2A Cyclin-dependent Kinase Inhibitor $2 \mathrm{~A}$

CDX2 Caudal Type Homeobox 2

$\mathrm{Cl}$ Confidence Interval

CV Cross Validation

\section{D}

DAXX Death Domain-associated Protein

DN Double Negative (ARX-/PDX1-)

DNA Deoxyribonucleic Acid

DP Double Positive (ARX+/PDX1+)

dpNETs Duodenopancreatic neuroendocrine tumors

DSS Disease Specific Survival

E

EGA European Genome-phenome Archive EUS Endoscopic Ultrasound

F

FFPE Formalin-Fixed Paraffin-Embedded

FISH Fluorescence in Situ Hybridization

FNA Fine Needle Aspiration

FNB Fine Needle Biopsy

FSG Fasting Serum Gastrin
G

G1-3 WHO grade 1-3

G-cell Gastrin-cell

GCGR Glucagon Receptor Gene

GCHN Glucagon Cell Hyperplasia and

Neoplasia

GEO Gene Expression Omnibus

GLP1 Glucagon Like Peptide 1

$\mathrm{H}$

H\&E Hematoxylin \& Eosin stained

H3K36me3 Histone 3 Lysine 36 Trimethy-

lation

HIF Hypoxia Inducible Factor

HPF High Power Field

IDAT Intensity Data File (methylation)

IHC Immunohistochemistry

IQR Interquartile Range

K

Ki-67 Marker of Proliferation Ki-67

KRAS Kirsten Ras Oncogene Homolog

L

LI Labeling Index

LNM Lymph Node Metastasis

LOH Loss of Heterozygosity

$M$

MCN Mucinous Cystic Neoplasm

MEN1 Multiple Endocrine Neoplasia 1

mTOR Mechanistic Target of Rapamycin

N

NET Neuroendocrine Tumor

NF-PanNET Non-Functional Pancreatic

Neuroendocrine Tumor

NF1 Neurofibromatosis Type 1 


\section{0 \\ OOB Out of Bag \\ P}

PanIN Pancreatic Intraepithelial Neoplasia

PanNEC Pancreatic neuroendocrine carcinoma

PanNET Pancreatic neuroendocrine tumor

PAX6/8 Paired Box Protein 6/8

PB Pancreatoblastoma

PDAC Pancreatic Ductal Adenocarcinoma

PDX1 Pancreatic and Duodenal Homeobox 1

PHLDA3 Pleckstrin Homology-Like Domain Family A type 3

PIK3CA Phosphatidylinositol-4,5-Bisphosphate 3-Kinase Catalytic Subunit Alpha

PPI Proton Pump Inhibitor

PTH Parathyroid Hormone

$\mathbf{R}$

RB1 Retinoblastoma Protein 1

RF Random Forest

RFS Relapse Free Survival

S

SATB2 Special AT-rich Sequence-binding Protein 2

SD Standard Deviation
SETD2 SET Domain Containing 2

SMAD4 Mothers Against Decapentaplegic Homolog 4

SNP Single-nucleotide Polymorphism

SPN Solid-pseudopapillary Neoplasm

\section{$T$}

t-SNE t-Distributed Stochastic Neighbor Embedding

TCGA The Cancer Genome Atlas

TMA Tissue Micro Array

TNM Tumor, Nodes, and Metastases

TP53 Tumor Protein P53

TSC 1/2 Tuberous Sclerosis Complex $1 / 2$ TTF1 Thyroid Transcription Factor 1

V

VEGF Vascular Endothelial Growth Factor VHL Von Hippel Lindau

\section{W}

WHO World Health Organisation

WB Washing Buffer

$Y$

YY1 Yin Yang 1

$Z$

ZES Zollinger Ellison Syndrome 


\section{NEDERLANDSE SAMENVATTING}

Goed gedifferentieerde neuro-endocriene tumoren zijn zeldzame tumoren met een incidentie van ongeveer 6 per 100.000 per jaar. Ze kunnen in elk weefsel ontstaan dat neuro-endocriene cellen bevat, maar ze komen het meeste voor in de longen, pancreas of darm (1). Neuro-endocriene tumoren worden gewoonlijk gekenmerkt door een trabeculaire (omslag proefschrift) of geneste morfologie, een granulair cytoplasma en een gespikkeld nucleair chromatine patroon (gelijkend op "zout en peper") (2, 3). Neuroendocriene tumoren worden "functionerend" genoemd als ze hormonen uitscheiden in een dusdanige mate dat ze symptomen bij een patiënt veroorzaken.

Ons begrip van de neuro-endocriene tumorigenese in duodenum en pancreas is met name gebaseerd op onze kennis van hoe de tumorigenese werkt in monogenetische neuroendocriene tumor syndromen, zoals Multipele Endocriene Neoplasie type 1 (MEN1), Von Hippel Lindau (VHL) en Glucagon Cel Hyperplasie en Neoplasie (GCHN) syndroom (4-7). In MEN1 en VHL-syndroom initieert verlies van het wildtype allel van het betreffende gen de tumorigenese. In GCHN-syndroom zorgt een reactieve hyperplasie voor een verhoogde kans op somatische mutaties en daarmee het ontstaan van neoplasieën.

Het is waarschijnlijk dat vergelijkbare mechanismen een rol spelen in de niet syndroomgerelateerde (sporadische) duodenopancreatische neuro-endocriene tumorigenese, aangezien regelmatig dezelfde mutaties (e.g., MEN1) gezien worden. Genoom sequentie bepalingen hebben de belangrijkste spelers in de sporadische pancreatische neuroendocriene tumorigenese geïdentificeerd, en somatische mutaties worden onder andere gezien in ARID1A, ATRX, CDKN1B, CDKN2A, DAXX, MEN1, SETD2, TP53, VHL, YY1 en genen uit de $\mathrm{mTOR}$ route (8-11). Desondanks is de exacte rol en volgorde van deze mutaties in de sporadische tumorigenese nog onbekend.

Er zijn enkele belangrijke klinische uitdagingen in de behandeling van patiënten met neuroendocriene tumoren. Neuro-endocriene tumoren uit de pancreas kennen de hoogste mortaliteit, omdat deze vaak naar de lever uitzaaien $(1,12,13)$. Een operatie kan voorkomen dat een tumor uitzaait, maar pancreaschirurgie gaat ook gepaard met een hoge morbiditeit en mortaliteit. De uitdaging ligt hier in het voorspellen van het gedrag van de tumor. Met name bij kleine tumoren weegt het lage risico op uitzaaiingen mogelijk niet op tegen het risico van pancreaschirurgie $(14,15)$. Verder is het na elke operatie de vraag met welke 
frequentie patiënten teruggezien moeten worden voor vervolgonderzoek. Vroege detectie van uitzaaiingen postoperatief kan gunstig zijn voor de behandeling, terwijl uitgebreid vervolgonderzoek bij patiënten met laag risico tumoren een onnodige belasting kan zijn. Biologische markers die de neiging tot uitzaaien van neuro-endocriene tumoren uit de pancreas kunnen voorspellen zijn daarom gewenst, en zouden zowel pre- als postoperatief de behandeling en monitoring kunnen verbeteren (16).

Een andere klinische uitdaging betreft patiënten die zich presenteren met een uitzaaiing van een neuro-endocriene tumor, waarbij het onduidelijk is waar de oorspronkelijke tumor zit. Kennis van de locatie en type tumor is noodzakelijk om de juiste behandeling te kiezen, maar is ook van belang om de prognose en vervolg strategie te bepalen (17). De huidige diagnostiek, bestaande uit radiologische beeldvorming aangevuld met immunohistochemische panels blijkt hierin regelmatig niet voldoende sensitief te zijn (18). Een universele test om de oorsprong van uitzaaiingen te bepalen zou daarom een belangrijke toevoeging zijn aan de diagnostische evaluatie van deze patiënten.

Om deze klinische vraagstukken op te lossen, gebruikt een patholoog traditioneel een combinatie van weefsel- en cel-morfologie en immunohistochemische kleuringen om tumoren te classificeren en stratificeren. Zoals duidelijk wordt uit hoofdstuk 2, is dit voldoende om verschillende veelvoorkomende soorten kanker uit de pancreas met hoge betrouwbaarheid te onderscheiden en daarmee de vervolgstrategie te bepalen. Helaas is het vaak lastig om neuro-endocriene tumoren uit de pancreas, maar ook uit andere organen juist te classificeren, omdat deze morfologisch en immunohistochemisch op elkaar lijken (18). Om die reden wordt er in dit proefschrift voor een andere invalshoek gekozen.

Het gedrag van cellen is het resultaat van selectieve genexpressie. Welke genen worden afgelezen wordt door de epigenetica bepaald. Epigenetische markeringen zorgen voor vormveranderingen van de gekoppelde DNA en eiwit structuren waardoor genen wel of niet toegankelijk zijn voor de transcriptie eiwitten (19). Een van de bekendere epigenetische markeringen is DNA methylatie, welke nauw samenhangt met andere epigenetische markeringen zoals histon modificaties. De combinatie van driedimensionale conformatieveranderingen zorgt voor epigenetische cel morfologie die specifiek is voor de differentiatie en het gedrag van een cel. Epigenetische cel/tumor morfologie ligt niet alleen aan de basis van de morfologische eigenschappen en eiwit expressie die door een patholoog kunnen worden waargenomen, maar reguleert ook de processen die normaal 
gesproken onzichtbaar zijn. Daardoor zijn epigenetische profielen zeer weefsel- en tumor-type specifiek (20), en worden ze op dit moment al gebruikt om hersentumoren te classificeren en de oorsprong te bepalen van niet-neuro-endocriene tumoren van onbekende primaire origine $(21,22)$.

Interessant genoeg zijn veel van de genen die gemuteerd zijn in neuro-endocriene tumoren (MEN1, ATRX, DAXX, ARID1A, SETD2 (23)) belangrijk in het onderhouden van de epigenetische markeringen. Dit zou kunnen betekenen dat epigenetische deregulatie een rol speelt in de neuro-endocriene tumorigenese, en mogelijk gebruikt kan worden om gedrag en type te voorspellen. Recent hebben meerdere studies dit vermoeden bevestigd, en zijn voor zowel pancreas, darm en longtumoren epigenetische subtypes ontdekt die prognostische waarde hebben $(9,24-28)$. Specifiek in de pancreas is gebleken dat niet-functionerende neuro-endocriene tumoren qua epigenetisch profiel lijken op normale alfa- en bèta-cellen (glucagon en insuline producerende cellen). De expressie van aristaless-related homeobox gene (ARX) en pancreatic and duodenal homeobox 1 (PDX1) werd als surrogaatmarker van deze respectievelijke alfa- en betaprofielen beschreven. Het bleek dat ARX-positieve/PDX1-negatieve en ARX-negatieve/PDX1-negatieve tumoren vaak uitzaaiden, terwijl ARX-negatieve/PDX1-positieve en ARX-positieve/PDX1-positieve tumoren juist meer indolent gedrag lieten zien $(24,28)$.

In het eerste deel van dit proefschrift zal verder worden ingegaan op de ontstaanswijze van neuro-endocriene tumoren, terwijl in het tweede deel de diagnostische en prognostische waarde van epigenetische tumor morfologie of surrogaatmarkers daarvan verder zal worden uitgezocht en worden vergeleken met bestaande markers.

\section{DEEL 1. DIFFERENTIATIE-SPECIFIEKE DUODENOPANCREATISCHE ROUTES VAN TUMORIGENESE}

De differentiatie en functie van een cel bepalen de vatbaarheid voor specifieke mutaties en routes van tumorigenese. Zoals samengevat in hoofdstuk 2, spelen mutaties in KRAS een centrale en initiërende rol in de sporadische ductale-cel tumorgenese, aangezien in zowel microscopische voorloper tumortjes als ductale adenocarcinomen mutaties van KRAS gevonden worden. KRAS mutaties worden daarentegen nooit gezien in de endocriene tumorigenese in de pancreas, die wordt gedomineerd door mutaties in tumor supressors MEN1 (40\%) en ATRX of DAXX (30\%) (8). Wederzijds exclusieve mutaties in $A T R X$ en DAXX zijn geassocieerd met een alternatieve verlenging van telomeren (ALT) (29). 
Regelmatig worden microscopische tumortjes ( $<5 \mathrm{~mm}$, microadenomen) met een neuroendocriene differentiatie gevonden in pancreasresecties. Het is echter onduidelijk of deze sporadische microadenomen reactief zijn of autonoom (i.e., of ze voorlopers zijn van neuroendocriene tumoren). Afwijkingen van MEN1, ATRX of DAXX of de aanwezigheid van ALT in microadenomen zouden kunnen suggereren dat ze autonome voorloper tumoren zijn. In hoofdstuk 3 hebben we aangetoond dat Menine, het eiwitproduct van MEN1, afwijkend tot expressie gebracht wordt in 14/19 (74\%) van de sporadische microadenomen. ATRX/ DAXX verlies en ALT werden niet gezien. Het is waarschijnlijk dat op zijn minst een deel van deze microadenomen somatische mutaties heeft in MEN1, aangezien we in een serie neuroendocriene tumoren uit de pancreas waarop een exoom sequentie analyse is uitgevoerd aantonen dat mutaties in MEN1 correleren met afwijkende eiwit expressie. Hiermee wordt waarschijnlijker dat sporadische microadenomen voorlopers zijn van neuro-endocriene tumoren. Het gebrek aan ATRX/DAXX verlies of ALT suggereert vervolgens dat deze de sporadische endocriene tumorigenese in de pancreas niet initiëren, en over het algemeen pas later een rol hebben. Dit is in overeenstemming met studies die beschrijven dat ALT vaker voorkomt in grotere neuro-endocriene tumoren, subclonaal aanwezig kan zijn, en dat neuro-endocriene tumoren in ATR-X syndroom niet beschreven zijn (kiembaan ATRXmutaties) (30-32). Verder bevestigen deze resultaten dat MEN1-syndroom een valide model van de sporadische tumorigenese in de pancreas is: alle MEN1-syndroom geassocieerde microadenomen hebben verlies van het gezonde MEN1 allel $(4,33)$, en ATRX/DAXX verlies en ALT worden alleen gezien grotere tumoren $(34,35)$.

In hoofdstuk 4 worden de klinische en pathologische karakteristieken van Glucagon Cel Hyperplasie en Neoplasie (GCHN) samengevat. Waar in MEN1-syndroom het verhoogde risico op neuro-endocriene tumoren uit de pancreas komt door een kiembaan mutatie in MEN1, is bij GCHN het toegenomen risico te wijten aan een toegenomen celdeling van alfa-cellen (glucagon producerende cellen). Door een defecte glucagon receptor in de lever, worden de ureagenese en gluconeogenese verstoord. Dit resulteert in verhoogde concentraties van aminozuren in het bloed, die de alfa-cellen aanzetten om te groeien (36). De proliferatie en hyperplasie zorgen voor een verhoogd risico op fouten door celdeling (37), waardoor het risico op neoplasie toeneemt. In hoofdstuk 5 bespreken we vervolgens mogelijke overeenkomsten van GCHN en de duodenale endocriene tumorigenese bij MEN1-syndroom. In tegenstelling tot de MEN1-syndroom gerelateerde neuro-endocriene tumoren in de pancreas, is het gezonde allel van MEN1 regelmatig nog aanwezig in MEN1-syndroom gerelateerde duodenale gastrinomen. Daarnaast is er ook 
vaak hyperplasie van de G-cellen (gastrine producerende cellen) in het duodenum. Bijna alle patiënten met MEN1 syndroom ontwikkelen gedurende hun leven hypercalciëmie door bijschildklier adenomen. Aangezien extracellulaire calcium levels gastrine secretie kunnen uitlokken, calcium door de calcium sensing receptor (CaSR) G-cellen kan aanzetten tot groei, en patiënten met MEN1-syndroom soms genezen van hypergastrinemie na een bijschildklier resectie, speculeren we dat hypercalciëmie van invloed kan zijn op het risico van gastrinomen in MEN1-syndroom (38).

\section{DEEL 2. EEN VERFIJNDE CLASSIFICATIE VAN NEURO- ENDOCRIENE TUMOREN DOOR MIDDEL VAN EPIGENETICA}

\subsection{Biologische markers van gedrag}

Het gedrag van neuro-endocriene tumoren uit de pancreas kan variëren van indolent tot zeer agressief. Een biologische marker die het gedrag kan voorspellen zou van waarde zijn in de pre- en postoperatieve chirurgische besluitvorming, met het doel om over- en onderbehandeling te voorkomen, en alleen patiënten intensief op te volgen na chirurgie als er een verhoogd risico op uitzaaiingen of een recidief is. Op dit moment worden tumor grootte, graad (op basis van delingsactiviteit) en de aanwezigheid van lymfeklier uitzaaiingen gebruikt om gedrag te voorspellen $(14,15)$. Desondanks kunnen kleine graad 1 tumoren uitzaaien (39), en kan het een uitdaging zijn om lymfeklier uitzaaiingen preoperatief op beeldvorming vast te stellen. Biologische markers die betrouwbaar gedrag kunnen voorspellen zouden daarom de basis van pre- en postoperatieve chirurgische besluitvorming sterk kunnen verbeteren.

Enkele veelbelovende nieuwe biologische markers met prognostische waarde zijn ALT, ATRX/DAXX en de epigenetische surrogaatmarkers ARX en PDX1 (24, 28-30, 40). Het was nog onbekend of deze nieuwe biologische markers preoperatief bepaald konden worden. In hoofdstuk 6 hebben we de betrouwbaarheid een preoperatieve bepaling van deze markers retrospectief in 13 cytologische preparaten en bijbehorende pancreas resecties vastgesteld. ALT, ARX en PDX1 konden met goede betrouwbaarheid worden bepaald (Kappa $>0,70)$. In vergelijking, tumor graad werd regelmatig onderschat (Kappa 0,28).

In hoofdstuk 7 hebben we ALT, ARX en PDX1 bepaald in indolente en uitzaaiende insulinomen. Insulinomen worden geopereerd om hypoglycemieen te verhelpen, en meestal (90\%) zijn patiënten genezen na deze operatie en krijgen ze geen uitzaaiingen. In ons cohort van 37 insulinomen hadden 5 patiënten lever uitzaaiingen. Alle insulinomen 
zonder uitzaaiingen waren klein $(<3 \mathrm{~cm})$, hadden geen ALT, en waren PDX1-positief/ARXnegatief (expressie van normale bèta-cel). Uitzaaiende insulinomen waren $>3 \mathrm{~cm}$, hadden in vier van de vijf gevallen ALT, en waren ARX-positief (vier waren ook PDX1-positief).

Door deze onverwachte resultaten overwogen wij dat uitzaaiende insulinomen voortkomen uit niet-functionerende neuro-endocriene tumoren met de novo insuline productie. In de eerste plaats waren de uitzaaiende insulinomen groot (gemiddeld 6,16 cm [SD 2,63]). Aangezien de diagnose pas plaatsvond toen patiënten hypoglycemische symptomen kregen, waren deze tumoren per definitie toen ze wat kleiner waren niet-functionerend. In de tweede plaats worden ATRX en DAXX-mutaties niet gezien bij sporadische insulinomen, terwijl deze relatief vaak voorkomen bij niet-functionerende neuro-endocriene tumoren uit de pancreas $(10,41)$. Het feit dat vier van de vijf uitzaaiende insulinomen ALT had, laat zien dat ze in hun route van tumorigenese meer op niet-functionerende neuro-endocriene tumoren uit de pancreas lijken. In de derde plaats is de expressie van ARX in alleen de uitzaaiende gevallen zeer opvallend, aangezien dit normaal gesproken alleen voorkomt in alfa-, epsilon- of gamma-cellen. $(24,28,42,43)$. Niet-functionerende neuro-endocriene tumoren uit de pancreas hebben regelmatig ARX-expressie.

In hoofdstuk 8 hebben we de postoperatieve associatie van recidief vrije overleving en ALT, ATRX/DAXX, ARX, PDX1 en klinisch-pathologische markers gevalideerd in een internationaal retrospectief cohort van 561 niet functionerende neuro-endocriene tumoren uit de pancreas zonder afstandsuitzaaiingen ten tijde van de pancreas operatie. ARX en PDX1 hadden hierbij onverwachts geen prognostische waarde. Hoewel ARX-positieve/ PDX1-negatieve tumoren geassocieerd waren met recidieven ten opzichte van PDX1positieve/ARX-negatieve tumoren, verdween deze associatie als ALT of ATRX/DAXX in een multivariate analyse werd meegewogen. Dit kan worden verklaard doordat tumoren met ALT vaker ARX positief zijn. In een multivariaat model waren alleen tumor grootte $>2 \mathrm{~cm}$, lymfeklier uitzaaiingen of ALT (vervangbaar door ATRX/DAXX verlies) voorspellend voor de kans op recidieven. In een subanalyse voor tumoren $\leq 2 \mathrm{~cm}$ zonder lymfeklier uitzaaiingen, was alleen de aanwezigheid van ALT geassocieerd met een toegenomen kans op recidieven postoperatief.

Dit is de eerste internationale studie die in een grote groep sporadische patiënten zonder bekende uitzaaiingen tijdens chirurgie uitgebreid onderzoek heeft verricht naar de prognostische waarde van biologische markers om zo recidief vrije overleving te 
voorspellen. Op elk moment gedurende follow-up, was het risico op een recidief in de groep met ALT-positieve tumoren 5,6x groter dan in de groep met ALT-negatieve tumoren, ook in de subanalyse van tumoren $\leq 2 \mathrm{~cm}$ zonder lymfeklier uitzaaiingen. Dit levert sterk bewijs op voor het belang om in de postoperatieve situatie ALT (of ATRX/DAXX) te bepalen in elke neuro-endocriene tumor uit de pancreas. Prospectieve studies moeten uitwijzen of ALT of ATRX/DAXX verlies ook waardevol is om patiënten met tumoren $\leq 2 \mathrm{~cm}$ te selecteren voor chirurgie.

\subsection{Het voorpellen van de oorsprong met endocriene differentiatie}

In hoofdstuk 8 is de prognostische waarde van ARX/PDX1, ALT en ATRX/DAXX ook onderzocht voor 107 pancreas neuro-endocriene tumor afstandsuitzaaiingen en 654 nonpancreas neuro-endocriene tumoren. Hoewel er geen prognostische waarde was in neuroendocriene tumoren die niet uit de pancreas kwamen, was de aanwezigheid van markers wel oorsprong specifiek. Vergelijkbaar met eerdere studies werd ATRX/DAXX verlies of ALT uitsluitend gezien in de pancreas en enkele neuro-endocriene tumoren uit de long (44, 45). ARX/PDX1 was positief in een proportie van de pancreas, maag, duodenum, appendix en rectum neuro-endocriene tumoren, maar negatief in de long, jejunum, en ileum neuro-endocriene tumoren. Voor ARX was dit nog niet beschreven, en voor PDX1 was dit consistent met de literatuur (46-50). Om deze reden hebben deze markers een mogelijk toekomstige rol in het bepalen van de oorsprong van uitzaaiingen van onbekende primaire origine.

Aangezien in hoofdstuk 8 de proportie van ARX en PDX1 positieve primaire neuroendocriene tumoren en uitzaaiingen vergelijkbaar was, vermoedden we dat de expressie van deze transcriptiefactoren klonaal doorgegeven wordt tussen primaire tumor en gerelateerde uitzaaiingen. In hoofdstuk 9 bevestigen we dit, en laten we zien dat 48 van de 50 uitzaaiingen van neuro-endocriene tumoren uit de pancreas vrijwel hetzelfde transcriptiefactor profiel hebben als de primaire tumor. Vervolgens gebruiken we dit gegeven om inzicht te krijgen in de relatie tussen primaire tumoren en uitzaaiingen in patiënten met MEN1-syndroom. Patiënten met MEN1-syndroom hebben vrijwel altijd meerdere primaire tumoren in de pancreas en het duodenum, en daarom is het lastig om te weten welke tumor exact de uitzaaiing heeft gegeven. Het bleek voor alle patiënten met hypergastrinemie, dat de lymfeklieren rondom de pancreaskop en duodenum zonder uitzondering een transcriptiefactor profiel hadden dat overeenkwam met de gastrinomen in het duodenum, en niet met de neuro-endocriene tumoren uit de pancreas. Deze 
duodenale gastrinomen zijn vaak microscopisch klein en kunnen preoperatief gemist worden. In dit kader is het van belang, zeker bij patiënten met hypergastrinemie, om lymfeklier uitzaaiingen niet zomaar aan neuro-endocriene tumoren uit de pancreas toe te schrijven. Verder onderstreept dit de noodzaak van een pancreatoduodenectomie om patiënten met gastrinomen en MEN1-syndroom te genezen.

In het laatste hoofdstuk (hoofdstuk 10) was het doel om een betere manier te vinden om de origine te vinden van neuro-endocriene tumor uitzaaiingen waarvan de primaire tumor onbekend is. Op dit moment kan de patholoog met immunohistochemische kleuringen (o.a. PDX1, hoofdstuk 8) een idee krijgen van waar een tumor vandaan komt, maar deze is in $<80 \%$ van de gevallen nauwkeurig. Zoals in de introductie van dit proefschrift vermeld, kan door middel van DNA methylatie de epigenetische tumor morfologie achterhaald worden, die zeer cel- en tumor-specifiek is. In dit onderzoek hebben we de neuro-endocriene tumoren die het vaakst uitzaaiingen geven (pancreas, dunne darm, long) onderzocht op DNA methylatie van het complete genoom $(1,24-26)$. Met een random forest model en logistische regressie hebben we een voorspelmodel (NEN-ID) gemaakt die met $>95 \%$ nauwkeurigheid de origine van een kan voorspellen in een cohort van 193 neuroendocriene tumoren. We laten de toepassing van dit model zien op een neuro-endocriene tumor van onbekende origine, en presenteren het model op een vrij te gebruiken website. 


\section{NEURO-ENDOCRIENE TUMORIGENESE IN DE PANCREAS: NIEUWE INZICHTEN DOOR DE EPIGENETISCHE TUMOR MORFOLOGIE}

Recente studies over endocriene differentiatie hebben een paradigma verschuiving in ons begrip van de neuro-endocriene tumorigenese in de pancreas veroorzaakt (24,28), ondanks het feit dat de onafhankelijke prognostische waarde van ARX/PDX1 in dit proefschrift niet werd bevestigd. Zo is de associatie van een alfa-cel subtype (of ARX-expressie) met MEN1 of ATRXIDAXX mutaties inmiddels meerdere malen in dit proefschrift (hoofdstuk 7,8 en 9) en de recente literatuur bevestigd $(24,28,51-54)$. Dit laat zien hoe de epigenetische tumor morfologie subtiele subgroepen kan ontdekken die in de traditionele histopathologische evaluatie gemist kunnen worden. Verder blijkt uit het meest recente onderzoek, dat ARX en PDX1 als surrogaatmarkers mogelijk een oversimplificatie zijn van een complex meerfasisch proces van tumorigenese.

Di Domenico et al. heeft een fylogenetische stamboom gemaakt op basis van DNA methylatie profielen om de relatie van 125 neuro-endocriene tumoren en alfa- en bètacellen vast te stellen (52). Er werden 3 groepen gevonden die vergelijkbaar waren met de groepen die eerder geïdentificeerd waren op basis van de chromosomale "copy number" variaties en DNA-sequentie analyses van het hele genoom door Hong et al. (25). De eerste subgroep bestond uit tumoren die een sterke overeenkomst vertoonden met alfacellen en een goede prognose hadden. Deze alfa-achtige tumoren hadden vaak MEN1 mutaties maar geen ATRX/DAXX mutaties, en gewoonlijk weinig copy number variaties. De tweede groep bestond uit tumoren met een sterke overeenkomst met bèta-cellen en hadden ook een goede prognose. De beta-achtige groep had geen MEN1/ATRX/DAXX mutaties, weinig copy number variaties en bestond voornamelijk uit insulinomen. De laatste groep bestond uit gededifferentieerde of "intermediate" tumoren die vaak een redelijke overeenkomst met alfa-cellen en enige overeenkomst met bèta-cellen hadden. Deze groep werd gekarakteriseerd door zowel MEN1 en ATRXIDAXX mutaties en een aanzienlijke hoeveelheid copy number deleties of amplificaties, en had een matige prognose. ARXexpressie werd gezien in zowel de alfa-achtige als de gedifferentieerde groep, waardoor duidelijk wordt dat ARX niet een marker is van tumor gedrag, maar eerder kan wijzen op een de route van tumorigenese (MEN1 + ATRX/DAXX mutaties).

De voorgestelde route van alpha-dedifferentiatie in de sporadische neuro-endocriene tumorigenese in de pancreas, waarin MEN1 mutaties de gemene deler zijn, sluit vreemd 
genoeg niet aan bij de observatie van bèta-achtige tumoren in MEN1-syndroom (28) (+hoofdstuk 9). Omdat sporadische insulinomen met MEN1 mutaties in de literatuur nog niet beschreven zijn $(10,41)$, is de observatie van insulinomen in MEN1-syndroom opvallend (55). Een mogelijke oorzaak hiervan is dat de studies die bèta-achtige niet-functionerende neuro-endocriene tumoren beschreven in patiënten met MEN1-syndroom hiervoor de immunohistochemische surrogaatmarkers ARX en PDX1 gebruikten, terwijl andere studies vaker van het complete transcriptoom/DNA methyloom gebruikten om de gelijkenis te bepalen. Het is mogelijk dat bèta-tumoren op basis van ARX/PDX1 expressie toch alfa-cel kenmerken hebben, die gemist worden met alleen ARX/PDX1 immunohistochemie (e.g., IRX2/MAFB(42)).

Een andere mogelijkheid, hoewel speculatief, is gebaseerd op het feit dat neuro-endocriene tumoren met mutaties in MEN1 uiteindelijk dedifferentiëren (de intermediate groep), en hiermee in een "immature" epigenetische staat komen. MEN1 is een belangrijke speler in het onderhouden van epigenetische markeringen (56), en het is daarmee begrijpelijk dat verlies van MEN1 een effect kan hebben op de differentiatie van een cel of tumor. Volgens de theorie van Waddington (57), kunnen veranderingen in het epigenetische landschap ervoor zorgen dat immature cellen een andere weg van differentiatie kiezen. Veranderingen in het epigenetische landschap kunnen veroorzaakt worden door de aan of afwezigheid van genexpressie (e.g., MEN1), maar ook door externe stimuli.

Patiënten met MEN1-syndroom hebben vrijwel altijd meerdere tumoren, die soms ook overmatig hormonen uitscheiden. Het is bekend dat endocriene cellen met elkaar communiceren. Zo bevordert prolactine de groei van zowel alfa- en bèta-cellen in zwangere vrouwen, en uit diabetes onderzoek blijkt dat GLP1 alfa-naar-bèta transdifferentiatie kan veroorzaken in endocriene cellen uit de pancreas $(58,59)$. Het is mogelijk dat door de multipele tumoren MEN1-syndroom, de immature neuro-endocriene tumoren met MEN1 verlies in de pancreas in een bepaalde staat van differentiatie (e.g., bèta) worden geduwd, afhankelijk van de hormonen (of externe stimuli) waaraan ze blootgesteld worden. In sporadische patiënten ontbreken deze externe stimuli en blijven deze tumoren in een immature staat met enige alfa-cel gelijkenis. 


\section{Toekomstige klinische- en onderzoeksperspectieven}

In dit proefschrift wordt de sterk prognostische waarde van ALT bevestigd in zowel grote als kleine niet-functionerende neuro-endocriene tumoren uit de pancreas. Daarnaast lijkt het van belang om ALT te bepalen in sporadische insulinomen. Om deze reden zou telomeer fluorescentie in situ hybridisatie of ATRX/DAXX immuunhistochemie moeten worden opgenomen in de histopathologische evaluatie van alle neuro-endocriene tumoren uit de pancreas, zowel in biopten als tumor in tumormateriaal na de operatie. Prospectieve studies zijn van groot belang om te beoordelen of ALT ook als preoperatieve marker van gedrag nuttig kan zijn om patiënten te selecteren voor chirurgie.

Onze toegenomen kennis van de op epigenetische profielen gebaseerde types neuroendocriene tumoren heeft een paradigma verschuiving veroorzaakt. Het heeft onze ogen geopend voor de (epi)genetische diversiteit in niet-functionerende neuro-endocriene tumoren uit de pancreas die voorheen werden gezien als een enkele groep. Verder leren we nog steeds van neuro-endocriene tumor syndromen, en zou in de toekomst de kennis van de groei stimulerende factoren zoals aminozuren in GCHN gebruikt kunnen worden om nieuwe behandelingen te ontwikkelen voor patiënten met neuro-endocriene tumoren.

Een van de redenen waarom we nu steeds meer de subtiele verschillen in neuro-endocriene tumor differentiatie kunnen waarderen, is de beschikbaarheid van relatief goedkope, betrouwbare testen om de epigenetische tumor morfologie te bepalen. Desondanks zijn er, met name door de zeldzaamheid van neuro-endocriene tumoren, nog veel uitdagingen. In de komende jaren is de belangrijkste uitdaging om het NEN-ID model uit te breiden zodat werkt voor alle neuro-endocriene tumoren. De toekomst zal leren of het mogelijk is om met DNA methylatie zelfs de prognose en therapeutische kwetsbaarheiden te bepalen. 


\section{BRONNEN}

1. Yao JC, Hassan M, Phan A, Dagohoy C, Leary C, Mares JE, et al. One hundred years after "carcinoid": epidemiology of and prognostic factors for neuroendocrine tumors in 35,825 cases in the United States. J Clin Oncol. 2008;26(18):3063-72.

2. Soga J, Tazawa K. Pathologic analysis of carcinoids. Histologic reevaluation of 62 cases. Cancer. 1971;28(4):990-8.

3. Lloyd RV, Osamura RY, Klöppel G, Rosai J. WHO Classification of Tumours of Endocrine Organs: International Agency for Research on Cancer; 2017.

4. Lubensky IA, Debelenko LV, Zhuang Z, Emmert-Buck MR, Dong Q, Chandrasekharappa S, et al. Allelic deletions on chromosome $11 \mathrm{q} 13$ in multiple tumors from individual MEN1 patients. Cancer Res. 1996;56(22):5272-8.

5. Lubensky IA, Pack S, Ault D, Vortmeyer AO, Libutti SK, Choyke PL, et al. Multiple neuroendocrine tumors of the pancreas in von Hippel-Lindau disease patients: histopathological and molecular genetic analysis. Am J Pathol. 1998;153(1):223-31.

6. Yu R, Nissen NN, Dhall D, Heaney AP. Nesidioblastosis and hyperplasia of alpha cells, microglucagonoma, and nonfunctioning islet cell tumor of the pancreas: review of the literature. Pancreas. 2008;36(4):428-31.

7. Henopp T, Anlauf M, Schmitt A, Schlenger R, Zalatnai A, Couvelard A, et al. Glucagon cell adenomatosis: a newly recog nized disease of the endocrine pancreas. J Clin Endocrinol Metab. 2009;94(1):213-7.

8. Scarpa A, Chang DK, Nones K, Corbo V, Patch A-M, Bailey $P$, et al. Whole-genome landscape of pancreatic neuroendocrine tumours. Nature. 2017;543(7643):65-71.

$9 . \quad$ Alcala N, Leblay N, Gabriel AAG, Mangiante L, Hervas $D$, Giffon T, et al. Integrative and comparative genomic analyses identify clinically relevant pulmonary carcinoid groups and unveil the supra-carcinoids. Nat Commun. 2019;10(1):3407.

10. Hong X, Qiao S, Li F, Wang W, Jiang R, Wu H, et al.

Whole-genome sequencing reveals distinct genetic bases for insulinomas and non-functional pancreatic neuroendocrine tumours: leading to a new classification system. Gut. 2020;69(5):877-87.

11. Francis JM, Kiezun A, Ramos AH, Serra S, Pedamallu

CS, Qian ZR, et al. Somatic mutation of CDKN1B in small intestine neuroendocrine tumors. Nat Genet. 2013;45(12):1483-6.

12. Man D, Wu J, Shen Z, Zhu X. Prognosis of patients with neuroendocrine tumor: a SEER database analysis. Cancer Manag Res. 2018;10:5629-38.

13. Keutgen XM, Schadde E, Pommier RF, Halfdanarson TR, Howe JR, Kebebew E. Metastatic neuroendocrine tumors of the gastrointestinal tract and pancreas: A surgeon's plea to centering attention on the liver. Semin Oncol. 2018;45(4):232-5.

14. Howe JR, Merchant NB, Conrad C, Keutgen XM, Hallet J, Drebin JA, et al. The North American Neuroendocrine Tumor Society Consensus Paper on the Surgical Management of Pancreatic Neuroendocrine Tumors. Pancreas. 2020;49(1):1-33. 15. Falconi M, Eriksson B, Kaltsas G, Bartsch DK, Capdevila J, Caplin M, et al. ENETS Consensus Guidelines Update for the Management of Patients with Functional Pancreatic Neuroendocrine Tumors and Non-Functional Pancreatic Neuroendocrine Tumors. Neuroendocrinology. 2016;103(2):153-71.

16. Jensen RT, Bodei L, Capdevila J, Couvelard A, Falconi
M, Glasberg S, et al. Unmet Needs in Functional and Nonfunctional Pancreatic Neuroendocrine Neoplasms. Neuroendocrinology. 2019;108(1):26-36.

17. Herrera-Martinez AD, Hofland LJ, Galvez Moreno MA Castano JP, de Herder WW, Feelders RA. Neuroendocrine neop-

lasms: current and potential diagnostic, predictive and prognostic markers. Endocr Relat Cancer. 2019;26(3):R157-R79.

18. Bellizzi AM. Immunohistochemistry in the diagnosis and classification of neuroendocrine neoplasms: what can brown do for you? Hum Pathol. 2020;96:8-33.

19. Lee DS, Luo C, Zhou J, Chandran S, Rivkin A, Bartlett $A$, et al. Simultaneous profiling of 3D genome structure and DNA methylation in single human cells. Nat Methods. 2019;16(10):9991006.

20. Lokk K, Modhukur V, Rajashekar B, Martens K, Magi R, Kolde $R$, et al. DNA methylome profiling of human tissues identifies global and tissue-specific methylation patterns. Genome Biol. 2014;15(4):r54.

21. Moran S, Martinez-Cardus A, Sayols S, Musulen E, Balana C, Estival-Gonzalez A, et al. Epigenetic profiling to classify cancer of unknown primary: a multicentre, retrospective analysis. Lancet Oncol. 2016;17(10):1386-95.

22. Capper D, Jones DTW, Sill M, Hovestadt V, Schrimpf $D$, Sturm D, et al. DNA methylation-based classification of central nervous system tumours. Nature. 2018;555(7697):469-74.

23. Pipinikas CP, Berner AM, Sposito T, Thirlwell C. The evolving (epi)genetic landscape of pancreatic neuroendocrine tumours. Endocr Relat Cancer. 2019;26(9):R519-R44.

24. Chan CS, Laddha SV, Lewis PW, Koletsky MS, Robzyk K, Da Silva E, et al. ATRX, DAXX or MEN1 mutant pancreatic neuroendocrine tumors are a distinct alpha-cell signature subgroup. Nat Commun. 2018;9(1):4158.

25. Karpathakis A, Dibra H, Pipinikas C, Feber A, Morris

T, Francis J, et al. Prognostic Impact of Novel Molecular Subtypes of Small Intestinal Neuroendocrine Tumor. Clin Cancer Res. 2016;22(1):250-8.

26. Laddha SV, da Silva EM, Robzyk K, Untch BR, Ke $\mathrm{H}$, Rekhtman N, et al. Integrative Genomic Characterization Identifies Molecular Subtypes of Lung Carcinoids. Cancer Res. 2019;79(17):4339-47.

27. Tirosh A, Killian JK, Petersen D, Zhu YJ, Walker RL, Blau JE, et al. Distinct DNA Methylation Signatures in Neuroendocrine Tumors Specific for Primary Site and Inherited Predisposition. J Clin Endocrinol Metab. 2020;105(10).

28. Cejas P, Drier Y, Dreijerink KMA, Brosens LAA, Deshpande $V$, Epstein $C B$, et al. Enhancer signatures stratify and predict outcomes of non-functional pancreatic neuroendocrine tumors. Nat Med. 2019;25(8):1260-5.

29. Heaphy CM, de Wilde RF, Jiao Y, Klein AP, Edil BH, Shi $C$, et al. Altered telomeres in tumors with ATRX and DAXX mutations. Science. 2011;333(6041):425.

30. Kim JY, Brosnan-Cashman JA, An S, Kim SJ, Song

KB, Kim MS, et al. Alternative Lengthening of Telomeres in Primary Pancreatic Neuroendocrine Tumors Is Associated with Aggressive Clinical Behavior and Poor Survival. Clin Cancer Res. 2017;23(6):1598-606. 
31. Marinoni I, Kurrer AS, Vassella E, Dettmer M, Rudolph T, Banz V, et al. Loss of DAXX and ATRX are associated with chromosome instability and reduced survival of patients with pancreatic neuroendocrine tumors. Gastroenterology. 2014;146(2):453-60 e5.

32. Masliah-Planchon J, Levy D, Heron D, Giuliano F, Badens C, Freneaux P, et al. Does ATRX germline variation predispose to osteosarcoma? Three additional cases of osteosarcoma in two ATR-X syndrome patients. Eur J Hum Genet. 2018;26(8):121721.

33. Debelenko LV, Zhuang Z, Emmert-Buck MR, Chandrasekharappa SC, Manickam P, Guru SC, et al. Allelic deletions on chromosome 11q13 in multiple endocrine neoplasia type 1 -associated and sporadic gastrinomas and pancreatic endocrine tumors. Cancer Res. 1997; 57(11):2238-43.

34. Perren A, Anlauf M, Henopp T, Rudolph T, Schmitt A, Raffel A, et al. Multiple endocrine neoplasia type 1 (MEN1): loss of one MEN1 allele in tumors and monohormonal endocrine cell clusters but not in islet hyperplasia of the pancreas. J Clin Endocrinol Metab. 2007;92(3):1118-28.

35. de Wilde RF, Heaphy CM, Maitra A, Meeker AK, Edil $\mathrm{BH}$, Wolfgang $\mathrm{CL}$, et al. Loss of ATRX or DAXX expression and concomitant acquisition of the alternative lengthening of telomeres phenotype are late events in a small subset of MEN-1 syndrome pancreatic neuroendocrine tumors. Mod Pathol. 2012;25(7):10339.

36. Sipos B, Sperveslage J, Anlauf M, Hoffmeister M, Henopp T, Buch S, et al. Glucagon cell hyperplasia and neoplasia with and without glucagon receptor mutations. J Clin Endocrinol Metab. 2015;100(5):E783-8.

37. Tomasetti C, Vogelstein B. Cancer etiology. Variation in cancer risk among tissues can be explained by the number of stem cell divisions. Science. 2015;347(6217):78-81.

38. Norton JA, Venzon DJ, Berna MJ, Alexander HR, Fraker DL, Libutti SK, et al. Prospective study of surgery for primary hyperparathyroidism (HPT) in multiple endocrine neoplasia-type 1 and Zollinger-Ellison syndrome: long-term outcome of a more virulent form of HPT. Ann Surg. 2008;247(3):501-10.

39. Pea A, Yu J, Marchionni L, Noe M, Luchini C, Pulvirenti A, et al. Genetic Analysis of Small Well-differentiated Pancreatic Neuroendocrine Tumors Identifies Subgroups With Differing Risks of Liver Metastases. Ann Surg. 2020;271(3):566-73.

40. Roy S, LaFramboise WA, Liu T-C, Cao D, Luvison A, Miller C, et al. Loss of Chromatin-Remodeling Proteins and/or CDKN2A Associates With Metastasis of Pancreatic Neuroendocrine Tumors and Reduced Patient Survival Times. Gastroenterology 2018;154(8):2060-3.e8.

41. Wang $H$, Bender $A$, Wang $P$, Karakose E, Inabnet WB Libutti SK, et al. Insights into beta cell regeneration for diabetes via integration of molecular landscapes in human insulinomas. Nat Commun. 2017;8(1):767.

42. Muraro MJ, Dharmadhikari G, Grun D, Groen N, Dielen T, Jansen E, et al. A Single-Cell Transcriptome Atlas of the Human Pancreas. Cell Syst. 2016;3(4):385-94 e3.

43. Baron M, Veres A, Wolock SL, Faust AL, Gaujoux R, Vetere A, et al. A Single-Cell Transcriptomic Map of the Human and Mouse Pancreas Reveals Inter- and Intra-cell Population Structure. Cell Syst. 2016;3(4):346-60 e4.
44. Dogeas E, Karagkounis G, Heaphy CM, Hirose K, Pawlik TM, Wolfgang CL, et al. Alternative lengthening of telomeres predicts site of origin in neuroendocrine tumor liver metastases. J Am Coll Surg. 2014;218(4):628-35.

45. Terra Md S, Xie Md Ph DH, Boland Md JM, Mansfield Md AS, Molina Md Ph DJ, Roden Md AC. Loss of ATRX expression predicts worse prognosis in pulmonary carcinoid tumors. Hum Pathol. 2019;94:78-85.

46. Srivastava A, Hornick JL. Immunohistochemical staining for CDX-2, PDX-1, NESP-55, and TTF-1 can help distinguish gastrointestinal carcinoid tumors from pancreatic endocrine and pulmonary carcinoid tumors. Am J Surg Pathol. 2009;33(4):626-32. 47. Hermann G, Konukiewitz B, Schmitt A, Perren A, Kloppel G. Hormonally defined pancreatic and duodenal neuroendocrine tumors differ in their transcription factor signatures: expression of ISL1, PDX1, NGN3, and CDX2. Virchows Arch. 2011; 459(2):147-54.

48. Yang Z, Klimstra DS, Hruban RH, Tang LH. Immunohistochemical Characterization of the Origins of Metastatic Well-differentiated Neuroendocrine Tumors to the Liver. Am J Surg Pathol. 2017;41(7):915-22.

49. Yang MX, Coates RF, Ambaye A, Cortright V, Mitchell JM, Buskey AM, et al. NKX2.2, PDX-1 and CDX-2 as potential biomarkers to differentiate well-differentiated neuroendocrine tumors. Biomark Res. 2018;6:15.

50. Gurevich LE, Proshchina AE, Voronkova IA, Ashevskaya VE, Korosteleva PA, Dolzhansky OV. [Differential diagnostic value of the expression of the transcription factor PDX-1 in neuroendocrine and non-neuroendocrine tumors of the pancreas and other organs]. Arkh Patol. 2019;81(5):11-21.

51. Boons G, Vandamme T, Ibrahim J, Roeyen G, Driessen A, Peeters D, et al. PDX1 DNA Methylation Distinguishes Two Subtypes of Pancreatic Neuroendocrine Neoplasms with a Different Prognosis. Cancers (Basel). 2020;12(6).

52. Di Domenico A, Pipinikas CP, Maire RS, Brautigam K, Simillion C, Dettmer MS, et al. Epigenetic landscape of pancreatic neuroendocrine tumours reveals distinct cells of origin and means of tumour progression. Commun Biol. 2020;3(1):740.

53. Simon T, Mamlouk S, Riemer P, Bormann F, Klinger B, Menne A, et al. An Integrative Genetic, Epigenetic and Proteomic Characterization of Pancreatic Neuroendocrine Neoplasms (PanNENs) defines Distinct Molecular Features of $a$ - and $\beta$-cell like Subgroups. bioRxiv. 2020:2020.06.12.146811.

54. Lakis V, Lawlor RT, Newell F, Patch AM, Mafficini A, Sadanandam A, et al. DNA methylation patterns identify subgroups of pancreatic neuroendocrine tumors with clinical association. Commun Biol. 2021;4(1):155.

55. van Beek DJ, Nell S, Verkooijen HM, Borel Rinkes IHM, Valk GD, Vriens MR, et al. Surgery for multiple endocrine neoplasia type 1-related insulinoma: long-term outcomes in a large international cohort. Br J Surg. 2020;107(11):1489-99.

56. Dreijerink KMA, Timmers HTM, Brown M. Twenty years of menin: emerging opportunities for restoration of transcriptional regulation in MEN1. Endocr Relat Cancer. 2017;24(10):T135-T45.

57. Waddington C. The Strategy of the Genes. Allen. Unwin, London; 1957.

58. Lee YS, Lee C, Choung JS, Jung HS, Jun HS. 
Glucagon-Like Peptide 1 Increases beta-Cell Regeneration by Promoting alpha- to beta-Cell Transdifferentiation. Diabetes. 2018;67(12):2601-14.

59. Zhang Z, Hu Y, Xu N, Zhou W, Yang L, Chen R, et al
A New Way for Beta Cell Neogenesis: Transdifferentiation from Alpha Cells Induced by Glucagon-Like Peptide 1. J Diabetes Res. 2019;2019:2583047. 


\section{CONTRIBUTING AUTHORS}

A

Muaz Aijazi

Chapter 8

Department of Medicine, University of Pittsburgh Medical Center, Pittsburgh, Pennsylvania, United States of America.

\section{Soyeon An}

Chapter 8

Department of Pathology, Incheon St. Mary's Hospital, College of Medicine, The Catholic University of Korea, Incheon, Republic of Korea.

\section{Mark A. Atkinson}

Chapter 3

Department of Pathology, College of Medicine, University of Florida, Gainesville, Florida, United States of America.

Department of Pediatrics, College of Medicine, University of Florida, Gainesville, Florida, United States of America.

\section{B}

\section{Nathan Bahary}

Chapter 8

Department of Medicine, University of Pittsburgh Medical Center, Pittsburgh, Pennsylvania, United States of America.

\section{Dirk-Jan van Beek}

Chapter 9

Department of Endocrine Surgical Oncology, University Medical Center Utrecht, Utrecht, The Netherlands.

\section{Samantha Bersani}

Chapter 8

Department of Diagnostics and Public Health, Section of Pathology, University of Verona, Verona, Italy.

\section{Inne H.M. Borel Rinkes}

Chapter 9

Department of Endocrine Surgical Onco-

logy, University Medical Center Utrecht, Utrecht, The Netherlands.

\section{Randall E. Brand}

Chapter 8

Department of Medicine, University of Pittsburgh Medical Center, Pittsburgh, Pennsylvania, United States of America.

\section{Lodewijk A.A. Brosens}

Chapter 2, 3, 4, 5, 6, 7, 8, 9, 10

Department of Pathology, University Medical Center Utrecht, Utrecht, The Netherlands.

Department of Pathology, The Sol Goldman Pancreatic Cancer Research Center, The Johns Hopkins University School of Medicine, Baltimore, Maryland, United States of America.

\section{Jacqueline Brosnan-Cashman}

Chapter 8

Department of Pathology, Johns Hopkins

Medical Institutions, Baltimore, Maryland, United States of America.

\section{C}

\section{Martha Campbell-Thompson}

Chapter 3

Department of Pathology, College of

Medicine, University of Florida, Gainesville, Florida, United States of America.

\section{Dengfeng Cao}

Chapter 8

Department of Pathology and Immunology, Washington University School of Medicine, St. Louis, Missouri, United States of America. 


\section{Jennifer S. Chennat}

Chapter 8

Department of Medicine, University of Pittsburgh Medical Center, Pittsburgh, Pennsylvania, United States of America.

\section{Sara Cingarlini}

Chapter 8

The Pancreas Institute, University and Hospi-

tal Trust of Verona, Verona, Italy.

Department of Medicine, Section of

Oncology, University and Hospital Trust of

Verona, Verona, Italy.

\section{Vincenzo Corbo}

Chapter 8

ARC-Net Centre for Applied Research on

Cancer, University and Hospital Trust of

Verona, Verona, Italy.

Department of Diagnostics and Public

Health, Section of Pathology, University of

Verona, Verona, Italy.

\section{D}

\section{Rohit Das}

Chapter 8

Department of Medicine, University of Pittsburgh Medical Center, Pittsburgh, Pennsylvania, United States of America.

\section{Koen M.A. Dreijerink}

Chapter 5, 6, 7, 8, 9, 10

Department of Endocrinology and Internal Medicine, Amsterdam University Medical Center, Amsterdam, The Netherlands. Department of Pathology, University Medical Center Utrecht, Utrecht, The Netherlands.

\section{E}

\section{Susanne van Eeden}

Chapter 7

Department of Pathology, Amsterdam
University Medical Center, Amsterdam, The Netherlands.

\section{Madelon van Emst}

Chapter 9

Department of Pathology, University Medical Center Utrecht, Utrecht, The Netherlands.

\section{$F$}

\section{Kenneth E. Fasanella}

Chapter 8

Department of Medicine, University of Pittsburgh Medical Center, Pittsburgh, Pennsylvania, United States of America.

\section{G}

\section{Christoph Geisenberger}

Chapter 10

Developmental Biology and Stem Cell Research, the Hubrecht Institute, Utrecht, The Netherlands.

\section{David Geller}

Chapter 8

Department of Surgery, University of Pittsburgh Medical Center, Pittsburgh, Pennsylvania, United States of America.

\section{$\mathrm{H}$}

\section{Xiaoli Han}

Chapter 8

Department of Pathology, University of Pittsburgh Medical Center, Pittsburgh, Pennsylvania, United States of America. 


\section{Christopher M. Heaphy}

Chapter 3, 6, 7

Department of Pathology, The Sol Goldman Pancreatic Cancer Research Center, The Johns Hopkins University School of Medicine, Baltimore, Maryland, United States of America.

Department of Medicine, Boston University School of Medicine, Boston, Massachusetts, United States of America.

\section{Michelle Heayn}

Chapter 8

Department of Pathology, University of Pittsburgh Medical Center, Pittsburgh, Pennsylvania, United States of America.

\section{Charlotte M. Heidsma}

Chapter 7, 8

Department of Surgery, Amsterdam University Medical Center, Amsterdam, The Netherlands.

\section{Melissa E. Hogg}

Chapter 8

Department of Surgery, NorthShore University Health System, Evanston, Illinois, United States of America.

\section{Seung-Mo Hong}

Chapter 8

Department of Pathology, Asan Medical Center, University of Ulsan College of Medicine, Seoul, Republic of Korea.

\section{Waki Hosoda}

Chapter 3

Department of Pathology, The Sol Goldman Pancreatic Cancer Research Center, The Johns Hopkins University School of Medicine, Baltimore, Maryland, United States of America.

\section{Ralph H. Hruban}

Chapter 2

Department of Pathology, The Sol Goldman Pancreatic Cancer Research Center, The Johns Hopkins University School of Medicine, Baltimore, Maryland, United States of America.

\section{K}

\section{Asif Khalid}

Chapter 8

Department of Medicine, University of Pittsburgh Medical Center, Pittsburgh, Pennsylvania, United States of America.

\section{Joo Young Kim}

Chapter 8

Department of Pathology, Nowon Eulji Medical Center, Eulji University, Seoul, Republic of Korea.

\section{Günter Klöppel}

Chapter 3

Department of Pathology, Technical University Munich, Munich, Germany.

\section{Aranxa S.M. Kok}

Chapter 9

Department of Pathology, University Medical Center Utrecht, Utrecht, The Netherlands.

\section{Björn Konukiewitz}

Chapter 3

Department of Pathology, Technical University Munich, Munich, Germany.

\section{Luca Landoni}

Chapter 8

The Pancreas Institute, University and Hospital Trust of Verona, Verona, Italy. 


\section{Rita T. Lawlor}

Chapter 8

ARC-Net Centre for Applied Research on Cancer, University and Hospital Trust of Verona, Verona, Italy.

\section{Kenneth Lee}

Chapter 8

Department of Surgery, University of Pittsburgh Medical Center, Pittsburgh, Pennsylvania, United States of America.

\section{Wendy W.J. de Leng}

Chapter 10

Department of Pathology, University Medical Center Utrecht, Utrecht, The Netherlands.

\section{Ta-Chiang Liu}

Chapter 8

Department of Pathology and Immunology, Washington University School of Medicine, St. Louis, Missouri, United States of America.

\section{Claudio Luchini}

Chapter 4, 8

Section of Pathology, Department of Diagnostics and Public Health, University and Hospital Trust of Verona, Verona, Italy.

\section{M}

\section{Andrea Mafficini}

Chapter 8

ARC-Net Centre for Applied Research on Cancer, University and Hospital Trust of Verona, Verona, Italy.

\section{J. Wallis Marsh}

Chapter 8

Department of Surgery, West Virginia University Health Sciences Center, Morgantown, West Virginia, United States of America.

\section{Kevin McGrath}

Chapter 8

Department of Medicine, University of Pittsburgh Medical Center, Pittsburgh, Pennsylvania, United States of America.

\section{Alan K. Meeker}

Chapter 3

Department of Pathology, The Sol Goldman Pancreatic Cancer Research Center, The Johns Hopkins University School of Medicine, Baltimore, Maryland, United States of America.

\section{Michele Milella}

Chapter 8

Department of Medicine, Section of Oncology, University and Hospital Trust of Verona, Verona, Italy.

\section{Massimo Milione}

Chapter 8

Department of Pathology, Fondazione IRCCS Istituto Nazionale dei Tumori di Milano, Milan, Italy.

\section{Leon M.G. Moons}

Chapter 6

Department of Gastroenterology, University Medical Center Utrecht, Utrecht, The Netherlands. 


\section{Folkert H.M. Morsink}

Chapter 6, 7, 8, 9, 10

Department of Pathology, University Medical Center Utrecht, Utrecht, The Netherlands.

\section{N}

\section{Michael A. Nalesnik}

Chapter 8

Department of Pathology, University of Pittsburgh Medical Center, Pittsburgh, Pennsylvania, United States of America.

\section{Els J.M. Nieveen van Dijkum}

Chapter 7,8

Department of Surgery, Amsterdam University Medical Center, Amsterdam, The Netherlands.

\section{Marina N. Nikiforova}

Chapter 8

Department of Pathology, University of Pittsburgh Medical Center, Pittsburgh, Pennsylvania, United States of America.

\section{Michaël Noë}

Chapter 3

Department of Pathology, University Medical Center Utrecht, Utrecht, The Netherlands.

Department of Pathology, The Sol Goldman Pancreatic Cancer Research Center, The Johns Hopkins University School of Medicine, Baltimore, Maryland, United States of America.

\section{O}

\section{Roderick J. O'Sullivan}

Chapter 8

Department of Pharmacology and Chemical Biology, University of Pittsburgh, Pittsburgh, Pennsylvania, United States of America.

\section{G. Johan A. Offerhaus}

Chapter 2, 5, 6, 7, 8, 9, 10

Department of Pathology, University Medical Center Utrecht, Utrecht, The Netherlands.

\section{Melanie C. Ongchin}

Chapter 8

Department of Surgery, University of Pittsburgh Medical Center, Pittsburgh, Pennsylvania, United States of America.

P

\section{Alessandro Paniccia}

Chapter 8

Department of Surgery, University of Pittsburgh Medical Center, Pittsburgh, Pennsylvania, United States of America.

\section{Antonio Pea}

Chapter 8

The Pancreas Institute, University and Hospital Trust of Verona, Verona, Italy.

\section{James F. Pingpank}

Chapter 8

Department of Surgery, University of Pittsburgh Medical Center, Pittsburgh, Pennsylvania, United States of America.

\section{Justin S. Poling}

Chapter 3

Department of Pathology, The Sol Goldman Pancreatic Cancer Research Center, The Johns Hopkins University School of Medicine, Baltimore, Maryland, United States of America. 


\section{Katherine E. Poruk}

Chapter 3

Department of Surgery, The Sol Goldman Pancreatic Cancer Research Center, The Johns Hopkins University School of Medicine, Baltimore, Maryland, United States of America.

\section{$\mathbf{R}$}

\section{Anthony Rizzo}

Chapter 3

Department of Pathology, The Sol Goldman Pancreatic Cancer Research Center, The Johns Hopkins University School of Medicine, Baltimore, Maryland, United States of America.

\section{Borislav Rusev}

Chapter 8

ARC-Net Centre for Applied Research on Cancer, University and Hospital Trust of Verona, Verona, Italy.

\section{$\mathrm{S}$}

\section{Roberto Salvia}

Chapter 8

The Pancreas Institute, University and Hospital Trust of Verona, Verona, Italy.

\section{Savreet Sarkaria}

Chapter 8

Department of Medicine, University of Pittsburgh Medical Center, Pittsburgh, Pennsylvania, United States of America.

\section{Aldo Scarpa}

Chapter 8

ARC-Net Centre for Applied Research on

Cancer, University and Hospital Trust of Verona, Verona, Italy.

Department of Diagnostics and Public Health, Section of Pathology, University of Verona, Verona, Italy.

\section{Willemien Schelhaas}

Chapter 7

Department of Pathology, Amsterdam

University Medical Center, Amsterdam, The Netherlands.

\section{Harkirat Singh}

Chapter 8

Department of Medicine, University of Pittsburgh Medical Center, Pittsburgh, Pennsylvania, United States of America.

\section{Aatur D. Singhi}

\section{Chapter 8}

Department of Pathology, University of Pittsburgh Medical Center, Pittsburgh, Pennsylvania, United States of America.

\section{Adam Slivka}

Chapter 8

Department of Medicine, University of Pittsburgh Medical Center, Pittsburgh, Pennsylvania, United States of America.

\section{You-Na Sung}

Chapter 8

Department of Pathology, Asan Medical

Center, University of Ulsan College of Medicine, Seoul, Republic of Korea.

\section{$\mathrm{T}$}

\section{Mark J.C. van Treijen}

Chapter 9

Department of Endocrine Oncology, University Medical Center Utrecht Cancer Center, Utrecht, The Netherlands. 


\section{V}

\section{Gerlof D. Valk}

Chapter 7, 8, 9, 10

Department of Endocrinology and Internal Medicine, University Medical Center Utrecht, Utrecht, The Netherlands.

Department of Endocrine Oncology, University Medical Center Utrecht Cancer Center, Utrecht, The Netherlands.

\section{Menno R. Vriens}

Chapter 7, 8, 9, 10

Department of Surgery, University Medical Center Utrecht, Utrecht, The Netherlands. Department of Endocrine Surgical Oncology, University Medical Center Utrecht, Utrecht, The Netherlands.

\section{W}

\section{Laura D. Wood}

Chapter 3

Department of Pathology, The Sol Goldman Pancreatic Cancer Research Center, The Johns Hopkins University School of Medicine, Baltimore, Maryland, United States of America.

Department of Oncology, The Sol Goldman Pancreatic Cancer Research Center, The Johns Hopkins University School of Medicine, Baltimore, Maryland, United States of America.

\section{Z}

\section{Herbert J. Zeh}

Chapter 8

Department of Clinical Sciences, Surgery, University of Texas Southwestern, Dallas, Texas, United States of America.

\section{Amer H. Zureikat}

Chapter 8

Department of Surgery, University of Pittsburgh Medical Center, Pittsburgh, Pennsylvania, United States of America. 


\section{CURRICULUM VITAE}

Wenzel Hackeng was born in Utrecht, the Netherlands on 13 January 1992. After two years Wenzel joined his parents in their move to San Diego, USA where he learned how to write. Aged 7, he moved back to the south of the Netherlands, to Cadier en Keer. After graduating cum laude from the Sint-Maartenscollege Gymnasium in Maastricht in 2010, Wenzel went abroad for a year to work and to travel. In 2011 he started medical school at the Utrecht University (UU), and obtained a Bachelor's degree in medicine in 2014. Next, he attended a 8-month research Traineeship at the Johns Hopkins Sol Goldman Pancreatic Cancer Research Center, supervised by dr. Lodewijk Brosens and dr. Laura Wood. Personal funding for this traineeship was obtained from USC Scholarship Fund, Nijbakker-Morra Stichting, the Lisa Waller-Hayes Foundation, and the KWF. Wenzel obtained his master's degree from the UU in 2018, which included final rotations in abdominal radiology, gastroenterology and internal medicine.

In 2018 Wenzel started as PhD student at the pathology department of the University Medical Center Utrecht, primarily to study the classification and tumorigenesis of gastrointestinal neuroendocrine tumors. During Wenzel's PhD studies, he attended several courses and trainings, including a biostatistics course, a basic course on regulations and organization for clinical investigators (BROK), and an advanced molecular pathology course. While presenting his research at the 31 st European Congress of Pathology in Nice, he was chosen best poster presenter of the section digestive disease pathology. Moreover, he received a NETRF Travel Scholarship 2020 for the 17th Annual ENETS Conference Barcelona, which was unfortunately cancelled due to COVID-19. Finally, together with his co-supervisor Lodewijk Brosens, Wenzel received competitive funding for his work on methylation-based prediction models from KWF (research grant 2020-1 "NEN-ID: predicting origin, prognosis and treatment vulnerabilities of metastatic neuroendocrine tumors using DNA methylation profiling) which includes funding for a full-time PhD student for three years. After finishing his full-time research position in march 2021, he started as medical Intern at the department of Gastroenterology and Internal Medicine, St. Antonius Ziekenhuis, Nieuwegein. In 2022, Wenzel will start his gastroenterology residency in the University Medical Center Utrecht or associated hospital in the region. 


\section{PUBLICATIONS IN THIS THESIS}

Hackeng WM, van Beek DJ, Kok ASM, van Emst M, Morsink FHM, van Treijen

MJC, Borel Rinkes IHM, Dreijerink KMA, Offerhaus GJA, Valk GD, Vriens MR, Brosens LAA. Metastatic patterns of duodenopancreatic neuroendocrine tumors in patients with multiple endocrine neoplasia type 1. American Journal of Surgical Pathology, 2021

Hackeng WM, Brosens LAA, Kim JY, O'Sullivan RJ, Sung YN, Liu TC, Cao D, Heayn, BrosnanCashman J, An S, Morsink FHM, Heidsma CM, Valk GD, Vriens MR, Nieveen van Dijkum EJM, Offerhaus GJA, Dreijerink KMA, Zeh HJ, Zureikat AH, Hogg ME, Lee K, Geller D, Marsh JW, Paniccia A, Ongchin MC, Pingpank JF, Bahary N, Aijazi M, Brand RE, Chennat JS, Das R, Fasanella KE, Khalid A, McGrath K, Sarkaria S, Singh H, Slivka S, Nalesnik MA, Han X, Nikiforova MN, Lawlor RT, Mafficini A, Rusev B, Corbo V, Luchini C, Bersani S, Pea A, Cingarlini S, Landoni L, Salvia R, Milione M, Milella M, Scarpa A, Hong SM, Heaphy CM, Singhi AD. Non-functional pancreatic neuroendocrine tumors: ATRX/DAXX and alternative lengthening of telomeres (ALT) assess prognosis independently from islet-cell subtype and tumor size. Gut, 2021

Hackeng WM, Dreijerink KMA, Offerhaus GJA, Brosens LAA. A Parathyroid-Gut Axis: Hypercalcemia and the Pathogenesis of Gastrinoma in Multiple Endocrine Neoplasia 1. Molecular Cancer Research, 2021

Hackeng WM, Dreijerink KMA, de Leng WWJ, Morsink FHM, Valk GD, Vriens MR, Offerhaus GJA, Geisenberger C, Brosens LAA. Genome methylation accurately predicts neuroendocrine tumor origin - an online tool. Clinical Cancer Research, 2020

Hackeng WM, Luchini C, Brosens LAA. Glucagon Cell Hyperplasia and Neoplasia. In: van Krieken J. (eds) Encyclopedia of Pathology. Encyclopedia of Pathology, 2020

Hackeng WM, Schelhaas W, Morsink FHM, Heidsma CM, van Eeden S, Valk GD, Vriens MR, Heaphy CM, Nieveen van Dijkum EJM, Offerhaus GJA, Dreijerink KMA, Brosens LAA. Alternative Lengthening of Telomeres and Differential Expression of Endocrine Transcription Factors Distinguish Metastatic and Non-metastatic Insulinomas. Endocrine Pathology, 2020 
Hackeng WM, Morsink FHM, Moons LM, Heaphy CM, Offerhaus GJA, Dreijerink KMA, Brosens LAA. Assessment of ARX expression, a novel biomarker for metastatic risk in pancreatic neuroendocrine tumors, in endoscopic ultrasound fine-needle aspiration. Diagnostic Cytopathology, 2019

Hackeng WM, Brosens LAA, Poruk KE, Noë M, Hosoda W, Poling JS, Rizzo A, CampbellThompson M, Atkinson MA, Konukiewitz B, Klöppel G, Heaphy CM, Meeker AK, Wood LD. Aberrant Menin expression is an early event in pancreatic neuroendocrine tumorigenesis. Human pathology, 2016

Hackeng WM, Hruban RH, Offerhaus GJA, Brosens LAA. Surgical and molecular pathology of pancreatic neoplasms. Diagnostic pathology, 2016 


\section{OTHER PUBLICATIONS}

Hackeng WM, Assi HA, Westerbeke FHM, Brosens LAA, Heaphy CM. Prognostic and predictive biomarkers for pancreatic neuroendocrine tumors. Submitted

Dreijerink KMA, Hackeng WM, Singhi AD, Heaphy CM, Brosens LAA. Clinical implications of cell-of-origin epigenetic characteristics in non-functional pancreatic neuroendocrine tumors. Submitted

Pflüger MJ, Griffin JF, Hackeng WM, Satomi K, Jun Y, Chianchiano P, Shin E, Lionheart G, Tsai $\mathrm{H}$, Wang H, Rezaee N, Burkhart RA, Cameron J, Thompson ED, Wolfgang CL, He J, Brosens LAA, Wood LD. "The Impact of Clinical and Pathological Features on Intraductal Papillary Mucinous Neoplasm Recurrence After Surgical Resection: Long-Term Follow-Up Analysis." Annals of Surgery, 2020

Van Den Broek MFM, De Laat JM, Van Leeuwaarde R, Van De Ven AC, De Herder WW, Dekkers OM, Drent ML, Kerstens MN, Bisschop PH, Havekes B, Hackeng WM, Brosens LAA, Vriens MR, Buikhuisen WA, Valk GD. The management of neuroendocrine tumors of the lung in MEN1: results from the Dutch MEN1 Study Group. The Journal of Clinical Endocrinology \& Metabolism. 2020

Noë M, Niknafs N, Fischer CG, Hackeng WM, Beleva Guthrie V, Hosoda W, Debeljak M, Papp E, Adleff V, White JR, Luchini C, Pea A, Scarpa A, Butturini G, Zamboni G, Castelli P, Hong SM, Yachida S, Hiraoka N, Gill AJ, Samra JS, Offerhaus GJA, Hoorens A, Verheij J, Jansen C, Adsay NV, Jiang W, Winter J, Albores-Saavedra J, Terris B, Thompson ED, Roberts NJ, Hruban RH, Karchin R, Scharpf RB, Brosens LAA, Velculescu VE, Wood LD. Genomic characterization of malignant progression in neoplastic pancreatic cysts. Nature communications, 2020

Kranendonk ME, Hackeng WM, Offerhaus GJA, Morsink FHM, Jonges GN, Groenewegen G, Krijtenburg PJ, Klümpen HJ, de Leng WWJ, Looijenga LHJ, Brosens LAA. The decisive role of molecular pathology in presumed somatic metastases of type II testicular germ cell tumors: report of 2 cases. Diagnostic pathology, 2020 
Dreijerink KMA, van Leeuwaarde RS, Hackeng WM, Giles RH, de Leng WWJ, Jutte PC, Suurmeijer AJH, van Nesselrooij BPM, Brosens LAA. Clear cell chondrosarcoma in Von Hippel-Lindau disease. Familial Cancer, 2020

Noë M, Hackeng WM, De Leng WWJ, Vergeer M, Vleggaar FP, Morsink FH, Wood LD, Hruban RH, Offerhaus GJA, Brosens LAA. Well-differentiated pancreatic neuroendocrine tumor in a patient with familial atypical multiple mole melanoma syndrome (FAMMM). The American journal of surgical pathology, 2019

Hackeng WM, de Guerre LEVM, Kuypers KC, Snoek AM, Morsink FH, Offerhaus GJA, Brosens LAA. Pseudomyxoma Peritonei After a Total Pancreatectomy for Intraductal Papillary Mucinous Neoplasm With Colloid Carcinoma in Lynch Syndrome. Pancreas, 2018

Hackeng WM, Montgomery EA, Giardiello FM, Singhi AD, Debeljak M, Eshleman JR, Vieth M, Offerhaus GJ, Wood LD, Brosens LA. Morphology and genetics of pyloric gland adenomas in familial adenomatous polyposis. Histopathology, 2017

Wood LD, Noë M, Hackeng WM, Brosens LAA, Bhaijee F, Debeljak M, Yu J, Suenaga M, Singhi AD, Zaheer A, Boyce A, Robinson C, Eshleman JR, Goggins MG, Hruban RH, Collins MT, Lennon AM, Montgomery EA. Patients with McCune-Albright syndrome have a broad spectrum of abnormalities in the gastrointestinal tract and pancreas. Virchows Archiv, 2017

Brosens LAA, Hackeng WM, Offerhaus GJA, Hruban RH, Wood LD. Pancreatic adenocarcinoma pathology: changing "landscape".

Journal of gastrointestinal oncology, 2015 


\section{ACKNOWLEDGEMENTS/DANKWOORD}

It has been six years since I have boarded a plane to Baltimore, which, in hindsight, represented the conception of my PhD studies. The last two and a half years have flown past. Although I worked my way through many deadlines, revisions, and occasional weekends, it never felt like a job, because it was always way too interesting and fun. I want to thank everyone who I have met along the way who has taught me techniques, statistics, research ethics, or has otherwise contributed the work described in this thesis.

Geachte prof. dr. Offerhaus, beste Johan. Jij bent niet alleen de inspirator van mijn onderzoeksavontuur, maar van inmiddels zoveel promovendi en pathologen, dat ik mij afvraag hoe de gastro-enterologische pathologie er in Nederland had uitgezien zonder jou... had het wel bestaan? Het was een eer dat ik aan "Groep-Offerhaus" mocht deelnemen. Jouw oprechte interesse en aanhoudende enthousiasme hebben mij ontzettend gemotiveerd; jouw jaloersmakende kennis en kritische vragen hebben mij scherp gehouden. Ook als ik eens in dwaze vertwijfeling naar het gras aan de overkant keek, was jij er om mij te helpen en om mee te denken. Met een zeer capabele opvolger, zal behalve de naam van de onderzoeksgroep, jouw wetenschappelijke nalatenschap nog lang de lijn van het onderzoek binnen de gastro-enterologische pathologie bepalen.

Geachte dr. Brosens, beste Lodewijk, toen wij elkaar nauwelijks kenden mocht ik in Baltimore de eerste dagen bij jullie komen logeren, natuurlijk ook aan Anne veel dank daarvoor. Die gastvrijheid en openheid is tekenend voor jou. Niet alleen stond de deur open, maar jij trok mij ook actief mee naar binnen. Zo introduceerde jij mij bij jouw wetenschappelijke connecties en liet je mij gelijk deelnemen aan samenwerkingen, lang voordat ik daadwerkelijk iets te bieden had. Zonder deze samenwerkingen was veel van het onderzoek in dit boekje niet mogelijk geweest. Later kon ik in het UMC altijd bij jou laagdrempelig binnenlopen: het kwam altijd uit. Jij hebt mij heel veel vrijheid en verantwoordelijkheid gegeven, en daar heb ik heel veel geluk mee gehad. Ik hoop nog lang onderzoek te mogen doen met "Groep-Brosens"!

Geachte dr. Dreijerink, beste Koen, ik heb genoten van onze onderzoeksbijeenkomsten. De wijze waarop jij jouw werk in de kliniek kan combineren met basaal wetenschappelijk onderzoek, waarin je bovendien steeds up-to-date alle literatuur kent, is indrukwekkend. Jouw "track change" suggesties heb ik gevreesd, maar inhoudelijk heb ik altijd veel aan 
jouw tips gehad.

Ik hoop zeker nog door te gaan met ons onderzoek samen en hoop nog veel van je te mogen leren.

Geachte leden van de leescommissie: prof. dr. Kranenburg, prof. dr. Speel, prof. dr. Valk, prof. dr. Vriens, en prof. dr. Vleggaar. Hartelijk dank voor het beoordelen van mijn manuscript.

Geachte heer Morsink, beste paranimf Folkert, jij hebt mij geleerd hoe ik wetenschappelijk onderzoek moest doen. Heel vroeg opstaan en goed voorbereiden, exact werken volgens de standard operating procedures, "check, dubbel check", en ter controle experimenten overdoen. Later kwamen ook "Folkerts handigheidjes", het fingerspitzengefühl en... "herhalen is balen". Bovenal vond ik het altijd heel erg gezellig met jou op het Pathology Research Lab en dat mis ik nu al. Gezellig met een koffietje praten over honden, wandelen of fietsen, of op de vrijmibo op een succesvolle week proosten. Ik hoop dat we dat nog veel zullen doen!

Geachte prof. dr. van Diest, beste Paul, dank voor het enthousiast steunen van ons onderzoek. Zonder jou was er geen NEN-ID voorspelmodel geweest!

Veel dank ook aan de wekelijkse meeting met de GE-pathologie groep, Marjon, Miangela, Wendy, Krijn, Lisa, Huiying, Michael, Hisham, en studenten Aranxa, Madelon, Nienke en Sangeeta. Ik heb altijd veel gehad aan de input en het was leuk en gezellig om elkaar wekelijks te zien! Anna Vera, heel veel plezier gewenst de komende jaren in deze leuke groep!

Ook Martine, en Floor, Willemien, wat was het een leuke start met jullie de eerste paar maanden door te brengen achter de flex-computers!

Mede NET onderzoekers: dr. prof. Valk en dr. prof. Vriens, Medard, veel dank voor jullie enthousiasme en de hulp met het klinisch duiden van onderzoeksresultaten. Dirk-Jan, gezellig over NETjes praten met een koffietje, laten we dat blijven doen! Christoph, thanks for your continuous support of my progression from R noob to nerd. Talya, it was great working together and to have a peek into your inspiring Hubrecht work. Charlotte en Els 
van het Amsterdam UMC, dank voor de fijne samenwerking!

PRL-vrienden, jammer dat jullie nooit zijn ingestapt op automaat koffie. Quirine, veel dank voor jouw felbegeerde plekje, fijn dat je spullen er ook nog liggen. Aernoud, fluorescentiekoning, veel dank voor de zo fijn voorgemengde DAPI. Emma T21-007. AIOS-pathologie is een goede dekmantel, kan jij pagina 5 oplossen? Lilian, de leukste buurvrouw die ook nog van Mustangs houdt, wat een geluk had ik daarmee. Carmen, altijd bij groen en recyclen... ik ga de Duitse muziek niet missen, maar de gezelligheid wel. Bregje, "Engels" gezellig en ook nog muzikaal, jammer dat jij halverwege vertrok naar boven. Azadeh, my kind new neighbor! Rachel, uitzonderlijk intelligente chirurg in spe, leuk om jou op het laatst nog te ontmoeten. Wisse, hopelijk wordt je snel promovendus zodat je mag blijven. Jan en Niels, het spijt mij dat ik zo vaak door jullie gesprekken heen moest lopen. Liling and Shuang, the party started after everybody went home, great times closing the lab together. In het hok boven: Sebastiaan (influencer), ik kijk naar jou op. Natalie, Mamma PA-P.A., jij houdt het clubje bij elkaar. Nog steeds jammer dat je voor de afzondering hebt gekozen beneden, Willem, het zal ergens goed voor zijn geweest. Betzabel, Floris, Selena, dank voor de gezellige lunches.

Roel, dank voor de geestige verhalen, maar ook zeer secure uitleg van de wondere wereld van de cameramicroscopen, en natuurlijk Ton Peeters, ik kan je tot mijn verdriet niet meer in het echt bedanken, er was geen betere (en strengere!) fluorescentie in situ hybridisatie leraar. Petra, Domenico, dank voor het aanleveren van de eindeloze dozen met blokjes en coupes. Marja, Shivanand, en Erwin, dank voor het snelle doorvoeren van de DNAextractie voor de methylatie assays. Nikolas, bedank voor jouw geduld en bedankt voor het meedenken met de Al-projecten. Leon, zonder jou was R nog steeds niet geïnstalleerd geweest. Henk, dank voor alle zeer belangrijke mails.

Dear dr. Wood, Laura, you are a wonderful and kind supervisor, thank you so much for the great time in your lab at Johns Hopkins, and for the responsibility you gave me to work out the Menin-microadenoma project. I would also like to thank prof. Hruban and dr. Montgomery for their supervising role, and all others which I met at Johns Hopkins, including dr. Meeker, Gemma, Waki, Jim, Katherine, Wenjie, Alexis, Marija, Marcia, and Eunice for their help, advice and the great time together. 
Dutchies! Anneline, Maite en Egbert, Marise en Ynte, Wendy, Patrick, Thomas: Baltimore Bike Parties, Rosemary garlic fries, en Associate Members of the Lithuanian Hall Association, wat was het ontzettend gezellig met jullie! Joost, ik zal nooit die mooie tijd aan 32nd street vergeten, Pete's Grill, brouwen, maar ook memorabele slowcook lessen, en de verstandige keuze midden in de nacht een hamburger halen op de fiets iets te ver buiten het "veilige" Charles village.

Chris and Aatur, I've really enjoyed working with you and I'm still very impressed on the great paper we've managed to write together. I hope it will be the first of many!

Antonio and Claudio, Quack. I'm happy knowing such a great surgeon and pathologist from Verona. Thank you for letting me visit your great hospital in Verona, and I hope to continue working together in the future!

Werkgroepje 14: Thijs, Janna, Annemar \& Flons. Dankzij jullie heb ik de communicatieen attitudelessen gehaald, en zonder jullie was van dit boekje dus ook nooit iets terechtgekomen!

James, my smartest and best friend, your enthusiasm for science continues to inspire me!

B3, lieve jaargenoten en andere Nerds, Guús en Poès, bedankt voor de geweldig gezellige avonden, een reden om terug te keren uit Baltimore. Rens en Tjeerd veel dank voor jullie Indesignadviezen! Vrienden van Houdini, zonder haring \& korenwijn, Miemel, en Schmull was ik verloren geweest.

Mijn lieve vrienden Govert, Ralph, Dirk, Olivier, en Koen, Mark, onze nooit verbroken cirkel. Als ik iemand ben, dan ben ik jullie. Mark, paranimf, veel dank dat jij mij wil helpen en steunen op dit cruciale moment. 
Broertje Julius, in de broertjesstrijd heb jij mij al eerder in het fysieke, maar recent ook nog eens in het professionele vlak verslagen. Jij hebt een onbreekbaar werkethos en wordt een steeds leukere gozer, maar wellicht moet je nu ook maar promoveren in de rechtsgeleerdheid?

Klein zusje Anemone, kie. Ik ben super benieuwd wat jij uiteindelijk besluit te gaan doen als arts, en wie weet zit jij sneller dan gedacht ook jouw proefschrift te verdedigen. Wat je ook gaat doen, het gaat zeker goedkomen, want je kan alles!

Lieve Opa en Oma, jullie zijn er tot mijn verdriet niet meer bij, bedankt voor jullie creativiteit en intelligentie, maar ook voor jullie koppigheid om in discussie te gaan.

Lieve Opi en Omi, bedankt voor jullie nauwkeurigheid en bevlogenheid in de wetenschap. Hopelijk mag ik als derde generatie promoveren aan de Universiteit Utrecht!

Lieve ouders, papa en mama, zonder jullie was er niets terecht gekomen van dit proefschrift. Op jonge leeftijd was jij, mama, al een kritische peer-reviewer van al mijn schrijven, en gelukkig nu nog steeds... Papa, jij liep streng mijn labjournaal na dat als ik als kind bijhield, vandaag praten wij over onderzoeksideeën terwijl we in de heuvels Shari en Frida uitlaten. Bedankt voor al jullie liefde, steun en inspiratie. Jullie zijn in nieuwsgierigheid, doorzettingsvermogen, maar ook in geest mijn grote voorbeeld.

Allerliefste Anne, wat ben ik ontzettend gelukkig dat ik met jou samen ben, in ons gezellige huisje met tuin met slingerlichtjes, maar nog geen kat. Ondanks lange dagen, en ook soms werk in de vrije uren, sta jij altijd voor mij klaar. Zonder jou had ik het niet volgehouden, dus je mag nooit meer bij mij weg! 

